\title{
Cyclic di-AMP homeostasis and osmoregulation in Listeria monocytogenes
}

\author{
Dissertation \\ for the award of the degree \\ „Doctor rerum naturalium“ (Dr. rer. nat.) \\ of the Georg-August-Universität Göttingen \\ within the doctoral program „Microbiology and Biochemistry “ \\ of the Georg-August University School of Science (GAUSS)
}

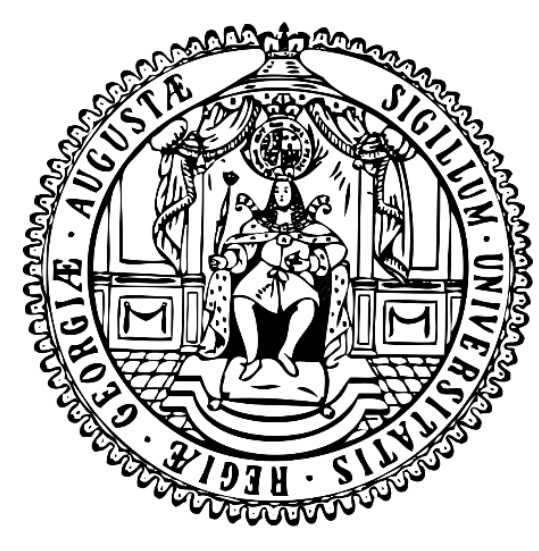

submitted by

Johannes Gibhardt

from Kassel

Göttingen, 2019 



\section{Examination board}

Thesis advisory committee

apl. Prof. Dr. Fabian M. Commichau

Institute of Microbiology and Genetics; Department of General Microbiology

Prof. Dr. Jörg Stülke

Institute of Microbiology and Genetics; Department of General Microbiology

Prof. Dr. Carsten Lüder

University Medical Center Göttingen; Department of Medical Microbiology

Additional members of the examination board

Referee:

Prof. Dr. Stefanie Pöggeler

Institute of Microbiology and Genetics; Department of Genetics of Eukaryotic Microorganisms

Co-referee:

Prof. Dr. Henning Urlaub

Max-Planck-Institute for Biophysical Chemistry; Bioanalytical Mass Spectrometry Group

$2^{\text {nd }}$ Co-referee:

Prof. Dr. Rolf Daniel

Institute of Microbiology and Genetics; Department of Genomic and Applied Microbiology

Date of oral examination: $2^{\text {nd }}$ of April 2019 
Cyclic di-AMP and osmoregulation in Listeria monocytogenes 
Preface

\section{Statement of authorship}

I hereby declare that the doctoral thesis entitled "Cyclic di-AMP homeostasis and osmoregulation in Listeria monocytogenes" has been written independently and with no other sources and aids than quoted. 
Cyclic di-AMP and osmoregulation in Listeria monocytogenes 


\section{Danksagung}

Zu aller erst möchte ich mich bei Dir bedanken, Fabian. Danke für inzwischen gute 7 Jahre der Unterstützung, dem Anhören meiner, oft um drei Ecken gedachten, Ideen und all dem, was ich in der Zeit von dir lernen durfte! Danke auch für den ein oder anderen unterhaltsamen Kneipenabend, mit dem doch häufig vorgekommenen "last call“ und allgemein der äußerst exzellenten Arbeitsatmosphäre in der HIF. Als nächstes möchte ich mich bei Jörg bedanken. Danke auch Dir für die Unterstützung in all den Jahren, den zahlreichen Tipps und auch den kritischen Fragen und Vorschlägen. Vielen Dank an dieser Stelle auch an euch beide für die Möglichkeit nicht nur meine Zeit als Doktorand, sondern bereits meine Zeit im Bachelor und Masterstudium in dieser außergewöhnlichen Abteilung zu verbringen! Ich möchte mich auch bei Dir, Carsten, bedanken, dass Du Teil meines Thesis Committee warst und dir die Zeit genommen hast meinen Fortschritt zu begutachten und mich zu beraten. An dieser Stelle möchte ich mich auch bei Stefanie Pöggeler, Henning Urlaub und Rolf Daniel für die Mitwirkung in meinem Prüfungskomitee bedanken.

Weiterhin möchte ich mich bei allen Kooperationspartnern bedanken die an den Projekten mitgewirkt haben. Allen voran Sven, Samuel, Jana, Alex, Anna-Lena, Annette, Vincent T. Lee, Volkhard Kaever, Uwe Völker und dem SPP1879 (hier vor allen Sebastian und Gerd für die unterhaltsamen Abende bei den Konferenzen).

Nun zu euch liebe AGS und AGC: Ich würde gerne zu jedem etwas schreiben, aber dies würde den Rahmen der Danksagung sprengen. Danke für die unglaublich geile Zeit! Wenn ich an meine Zeit als Bachelor, Master und anfänglicher PhD Student zurück denke muss ich sicherlich den „alten Hasen“ Katrin, Steffi, Joni, Miriam, Dodo, Chris, den Jans, Daniel, Lorena, Nora, Ingrid, Raphael und Bingyao für alles danken! An dieser Stelle darf natürlich auch Christina, Sabine, Silvia, Julia und Andrea nicht vergessen werden. Ohne euch würde mit Sicherheit nicht viel in der Abteilung passieren. Vor allem der hilfreiche Input von Christina und Sabine waren eine große Hilfe, sowie die angedrohten „Peitschenhiebe“ von Silvia ;-). Natürlich darf man auch die "jungen Küken“, vor allem Larissa, Martin B\&W, Neil, Björn, Patrick und Mengyi nicht vergessen. Nicht nur ihr habt, hoffentlich, etwas von mir gelernt, sondern ich auch von euch und ihr alle habt auf unterschiedliche Art zu dieser Arbeit beigetragen und auch dafür gesorgt, dass man immer wieder gerne zur Arbeit gekommen ist! Ach Leute, wir hatten schon eine überragende Zeit! Wenn ich nur an den ein oder anderen „Pizza \& Fußballabend“ zurückdenke, der traditionell aus dem Ruder gelaufen ist oder manch böses Erwachen, dann vermisse ich die Zeit schon jetzt! Ich werde euch alle vermissen oder tue dies bereits. Danke für alles!

Anika und Cedric, wir haben damals, vor bereits sieben Jahren, zusammen unsere Bachelorarbeit angefangen. Später kam dann die Master und nun schließlich die Doktorarbeit... wer hätte das gedacht? Danke euch für die überragende Zeit! Ich bin sicher wir bringen das Ganze zu einem erinnerungswürdigen Abschluss!

Natürlich möchte ich auch meinen ganzen Studenten danken, denen ich die Ehre hatte in den Jahren (hoffentlich) das ein oder andere beizubringen. Danke Gregor, Ole, Julian, Jasmin, Birthe, Lisa, Veronika, Sandra, Judith, Tariq und Lars! Ich habe auch etwas an Erfahrung von euch mitgenommen. Vor allem euch, Gregor, Ole und Julian möchte ich für die sehr unterhaltsame Zeit inund außerhalb des Labors danken. Ob Kneipenabend, dass ein oder andere Bier in der Abteilung oder der spontane Alpenmax besuch nachts um halb 2, ich hatte viel Spaß euch „zu betreuen“ ;-).

Zu guter Letzt möchte ich den Menschen danken die mir am meisten am Herzen liegen, meinen Freunden und meiner Familie. Worte können nicht beschreiben, was ihr mir bedeutet! 
Mädels und Jungs, ich danke euch für die geilen Jahre, sei es die inzwischen weit zurückliegende Schulabschlusszeit, die Zeit als frisch gebackener Student oder den ein oder anderen Urlaub. All diese Erinnerungen sind nur besonders, weil wir sie zusammen erlebt haben! An dieser Stelle einen besonderen Dank auch an all die Leute mit denen ich das Vergnügen hatte das ein oder andere Abenteuer an fremden Orten zu erleben oder Sie dort kennen zu lernen!

Wie sagte sogar Erich Kästner? „Toren bereisen in fremden Ländern die Museen, Weise gehen in die Tavernen“.

Seien es Kurztrips nach Hamburg, Frankfurt, Leipzig oder Städtetrips nach Barcelona (mit nassen Füßen), Madrid, Prag (noch nie so viel Spaß beim „Fahrradfahren“ gehabt), Mailand (immer diese Stadiontouren...), Turin, Zürich (die spinnen doch, was die Bierpreise angeht...), St. Petersburg (verrückte Iren und die weiße Nächte, прекрасно!), Moskau (Old School Pub und очень вкусный хачапури!), Susdal (dieser Met (медовуха) und dieser Ausblick, unbeschreiblich!), New York, Boston, Miami $\left(27^{\circ} \mathrm{C}\right.$ im November und ein kühles IPA, was will man mehr?), Key West (ein Wort, paradiesisch!), Orlando, Brügge, Brüssel (und die tausend Biere), London (und die verrückte Dart WM), und, und, und... Wir hatten eine geile Zeit und ich freue mich schon auf das nächste Abenteuer ;-). Danke Katha und Kaddah, Johannes und David, Mike, Hoffi, Anja, Annika, Chris, Marcel, Nils, Stephan, geborener Stelmecke ;-), Martin, Micha, Paddy, Robin, Borschti, Kevin, Sabrina, Daniel, Bierwirth, Kimi, Guilia, Nadine und der kleine Stubsi Jr., äh Mika ;-), ... Danke für alles!

Nein euch habe ich nicht vergessen ;-) Mein besonderer Dank gilt euch, liebes Wolfrudel! Kalle \& Tobi, danke! Danke für einfach so viele geile Augenblicke und Erinnerungen! Ich wüsste nicht wo ich heute ohne euch wäre und ich hoffe diese Freundschaft wird ein Leben lang halten! Ich höre hier jetzt lieber auf, sonst schreibe ich weitere 170 Seiten...

Zu guter Letzt möchte ich euch danken, liebe Familie! Danke euch allen, Martin, Florian, Pate Carsten, Godi Karin, Luis \& Paul, Silvia, Ludmilla, Heinz-Jürgen, Dieter, Christa, Juliane, Katrin, Lena, Merle, Rudi, Anchen, Stephan, Simone, Daniela, Tom, ... Danke!

Omas Elisabeth und Erika, Opas Heinrich und Henner, ich danke euch für alles was ihr in den 28 Jahren für mich gemacht habt. „Öpchen“ Henner, du wirst sehr vermisst! Mama, Papa und Christine, Worte können nicht ausdrücken wie dankbar ich euch bin und wie wichtig ihr mir seid! Danke für alles!

Diese Arbeit widme ich euch! 


\section{Table of contents}

Examination board

Thesis advisory committee I

Additional members of the examination board ।

Statement of authorship III

Danksagung v v

Table of contents VII

Summary IX IX

Lists of abbreviations $\quad$ XI

1. Introduction 1

$\begin{array}{ll}\text { Osmoregulation in bacteria } & 1\end{array}$

Sensing of changes in osmolarity by bacteria 3

The nucleotide second messenger c-di-AMP

$\begin{array}{ll}\text { Synthesis and degradation of c-di-AMP } & 6\end{array}$

$\begin{array}{lr}\text { The signaling network of c-di-AMP } & 8\end{array}$

Listeria monocytogenes and c-di-AMP

$\begin{array}{ll}\text { Objective of this thesis } & 14\end{array}$

2. The YbbR domain-containing protein CdaR regulates diadenylate cyclase activity of CdaA 15

Abstract $\quad 15$

$\begin{array}{ll}\text { Introduction } & 16\end{array}$

$\begin{array}{ll}\text { Experimental procedures } & 17\end{array}$

Results $\quad 23$

$\begin{array}{ll}\text { Discussion } & \mathbf{3 0}\end{array}$

3. Characterization of c-di-AMP-controlled potassium transporters of Listeria monocytogenes

35

Introduction $\quad 36$

$\begin{array}{ll}\text { Experimental procedures } & 37\end{array}$

Results $\quad 41$

$\begin{array}{ll}\text { Discussion } & 48\end{array}$

4. Global effects of c-di-AMP on gene expression and protein biosynthesis in Listeria monocytogenes

Abstract $\quad 51$

Introduction

Experimental procedures $\quad 53$

Results 
Discussion

5. The inhibitory effect of DNA on the activity of the diadenylate cyclase DisA of Bacillus subtilis is relieved by ions

Abstract

Introduction

Experimental Procedures

Results

Discussion

6. The sRNA rli31 affects lysozyme resistance, motility and gene expression in Listeria monocytogenes

Abstract

Introduction

Experimental Procedures 83

Results 86

Discussion

\section{Discussion}

Regulation of diadenylate cyclase activity

Regulation of CdaA by CdaR

The role of the YbbR domains of the diadenylate cyclase regulator CdaR

Regulation of other diadenylate cyclases

c-di-AMP-regulated osmotic homeostasis in Listeria monocytogenes

104

c-di-AMP affects global gene expression and protein biosynthesis

106

Outlook

109

8. Supplementary Data

Chapter 2

Chapter 4

Chapter 5

Chapter 6

9. References

10. Appendix

xxxV

11. Curriculum vitae

xxxix List of publications 


\section{Summary}

The second messenger cyclic diadenosine monophosphate (c-di-AMP) is essential for most of the bacteria synthesizing the nucleotide. c-di-AMP is produced by diadenylate cyclases (DACs) and degraded by specific phosphodiesterases (PDEs). c-di-AMP is involved in the control of different cellular processes, such as cell wall metabolism, carbon metabolism and osmoregulation. Osmoregulation is the cause for the essentiality of c-di-AMP, because regulation of osmolyte uptake systems has been shown to be affected by the second messenger. Hence, c-di-AMP is a major regulator of osmotic homeostasis in bacteria. The Gram-positive human pathogen Listeria monocytogenes possesses the DAC CdaA and the PDEs PdeA and PgpH for c-di-AMP synthesis and degradation, respectively. The CdaA-type of DAC is widespread in firmicute pathogenic bacteria and therefore a prime target for the development of novel antibiotics. The gene encoding CdaA is conserved with the genes $c d a R$ and $g / m M$, encoding the protein CdaR and the phosphoglucosamine mutase GImM, respectively. It is however unclear, how bacteria modulate c-di-AMP concentrations in response to osmotic changes and how c di AMP affects important osmotic adaptation processes, such as potassium transport, in L. monocytogenes. Here, novel insights on the regulation of DAC activity and the impact of c-di-AMP on cellular processes are elucidated. Synthesis and degradation of c-di-AMP have to be tightly adjusted, in dependency of the external osmolarity to maintain an isosmotic intracellular environment. In concert with modulations of the cell wall metabolism this subsequently results in maintaining a balanced turgor pressure. Hence, the proteins CdaR and GImM are prime targets to modulate CdaA activity. CdaR consists of an $\mathrm{N}$-terminal transmembrane domain and four YbbR domains of unknown function. It is shown that $\mathrm{CdaR}$, like CdaA, is a membrane protein, with the four YbbR domains located outside of the cell. Moreover, a $\Delta c d a R$ mutant shows defects in the adaptation to osmotic stress and an altered intracellular c-di-AMP concentration. For normal regulation of $\mathrm{CdaA}$ activity upon osmotic shock, both, the membrane localization and the presence of the $\mathrm{YbbR}$ domains are required. It is furthermore shown that both $\mathrm{CdaR}$ and $\mathrm{GlmM}$ are able to interact with $\mathrm{CdaA}$ and inhibit its activity in vivo. This highlights that CdaA probably integrates clues, signaling changes in osmolarity and cell wall biosynthesis, via its two regulators CdaR and GImM, respectively. Additionally, protein-protein interaction studies, show possible interactions between CdaAR and the PDEs, indicating cross-talk of synthesis and degradation machineries and local signaling of c-di-AMP. Moreover, using directed and undirected approaches, novel targets in L. monocytogenes that might be regulated by c-di-AMP are investigated. The proteins KtrCD and KimA of $L$. monocytogenes are shown to possess potassium transporter activity and both, KtrCD and KimA are demonstrated to be inhibited by c-di-AMP in vivo. $\mathrm{KtrC}$, furthermore, binds the nucleotide in vitro. The analysis of changes in global gene expression and protein synthesis demonstrates a broad impact of c-di-AMP on L. monocytogenes. Important cellular processes, such as the central metabolism, the regulation of transport processes and motility, as well as cell wall remodeling are implicated. Investigations of the connection between c-di-AMP signaling and alterations of the cell wall, additionally, demonstrated that L. monocytogenes rapidly adapts to the muralytic enzyme lysozyme by acquiring mutations in the promoter of the SRNA rli31, which has been shown to affect expression of cell wall modifying enzymes. To conclude, in the present study regulatory processes affecting c-di-AMP synthesis by changes in the osmolarity and the impact of c-di-AMP on global gene expression, protein biosynthesis, cell wall modifications and especially its impact on osmohomeostasis in the human pathogen L. monocytogenes, are investigated. The gained knowledge will help to understand the mechanisms Firmicutes use to sense changes in osmolarity and highlights mechanisms they use to adapt. Understanding regulation of DAC activity eventually can lead to the development of novel antibiotic to treat infections by these increasingly multi antibiotic resistant pathogens. 
Cyclic di-AMP and osmoregulation in Listeria monocytogenes 


\section{Lists of abbreviations}

\begin{tabular}{|c|c|}
\hline Abbreviation & Meaning \\
\hline$\%(v / v)$ & $\%$ (volume/volume) \\
\hline$\%(w / v)$ & \& (weight/volume) \\
\hline (p)ppGpp & guanosine penta -/ tetraphosphate \\
\hline$a d$ & up to \\
\hline ADP & adenosine diphosphate \\
\hline amp & ampicillin \\
\hline AMP & adenosine monophosphate \\
\hline AP & alkaline phosphatase \\
\hline ATP & adenosine triphosphate \\
\hline B. & Bacillus \\
\hline $\mathrm{BHI}$ & Brain Heart Infusion \\
\hline C & carbon \\
\hline CAA & casamino acids \\
\hline CAMP & cyclic adenosine monophosphate \\
\hline $\mathrm{CdaA} / \mathrm{cdaA}$ & cyclic di-AMP synthase A / -gene \\
\hline $\mathrm{CdaR} / \mathrm{cdaR}$ & cyclic di-AMP synthase regulator / -gene \\
\hline CdaS / cdas & B. subtilis sporulation specific cyclic di-AMP synthase / -gene \\
\hline c-di-AMP & cyclic di-adenosine monophosphate \\
\hline c-di-GMP & cyclic di-guanosine monophosphate \\
\hline cDNA & chromosomal DNA \\
\hline DAC & diadenylate cyclase \\
\hline $\mathrm{cm}$ & chloramphenicol \\
\hline DarA / darA & c-di-AMP receptor A \\
\hline DisA / disA & B. subtilis DNA integrity scanning protein A / -gene \\
\hline DNA & desoxyribonucleic acid \\
\hline dNTP & desoxyribonucleotide \\
\hline E. & Escherichia \\
\hline e.g. & exempli gratia \\
\hline EDTA & ethylenediaminetetraacetic acid \\
\hline erm & erythromycin \\
\hline et al. & et alia; and others \\
\hline Fig. & figure \\
\hline Fwd. & forward \\
\hline $\mathrm{GImM} / \mathrm{g} / \mathrm{mM}$ & phosphoglucosamine mutase / -gene \\
\hline $\mathrm{HhH}$ & helix-hairpin-helix \\
\hline HIF & high impact factory \\
\hline IPTG & isopropyl $\beta$-D-1-thiogalactopyranoside \\
\hline K & potassium \\
\hline kan & kanamycin \\
\hline Ktr / ktr & potassium transporter / -gene \\
\hline L. & Listeria or in some cases Lactococcus \\
\hline LB & lysogeny broth \\
\hline L-form & cell wall less form \\
\hline LSM & Listeria synthetic medium \\
\hline MCS & multiple cloning site \\
\hline
\end{tabular}




\begin{tabular}{|c|c|}
\hline Abbreviation & Meaning \\
\hline MDR & multi drug resistance \\
\hline$m G f p / m g f p$ & monomeric green fluorescent protein / -gene \\
\hline MOPS & 3-(N-morpholino)propanesulfonic acid \\
\hline mRNA & messenger RNA \\
\hline $\mathrm{N}$ & nitrogen \\
\hline $\mathrm{Na}$ & sodium \\
\hline NAG & $\mathrm{N}$-acetylglucosamine \\
\hline NAM & $\mathrm{N}$-acetylmuramic acid \\
\hline$O D_{x}$ & optical density at a wavelength of $\lambda=x \mathrm{~nm}$ \\
\hline ORF & open reading frame \\
\hline$P$ & promoter \\
\hline $\mathrm{p}$ & plasmid \\
\hline pApA & phosphoadenylyl adenosine \\
\hline PASTA & PBP and serine/threonine kinase associated \\
\hline PCR & polymerase chain reaction \\
\hline PDE & phosphodiesterase \\
\hline PdeA / pdeA & phosphodiesterase A / -gene \\
\hline penG & penicillin G \\
\hline PG & peptidoglycan \\
\hline $\mathrm{PgpH} / \mathrm{pgpH}$ & HD-domain phosphodiesterase / -gene \\
\hline $\mathrm{pH}$ & power of hydrogen \\
\hline$P_{i i}$ & inorganic pyrophosphate \\
\hline PrfA / prfA & positive regulatory factor $\mathrm{A}$ / -gene \\
\hline primer & oligonucleotide \\
\hline RBS & ribosomal binding site / Shine-Dalgarno sequence \\
\hline Rev. & reverse \\
\hline RNA & ribonucleic acid \\
\hline RNase & ribonuclease \\
\hline S & supplementary \\
\hline S. & Staphylococcus or Streptococcus \\
\hline SDS & sodium dodecyl sulfate \\
\hline SOE & splicing by overhang extension \\
\hline T4 & T4 phage \\
\hline Tab. & table \\
\hline TM & transmembrane \\
\hline TRIS & Tris(hydroxymethyl)aminomethane \\
\hline tRNA & transfer RNA \\
\hline UTR & untranslated region \\
\hline WGS & whole genome sequencing (illumina sequencing) \\
\hline wt & wild type \\
\hline X-Gal & 5-bromo-4-chloro-3-indolyl- $\beta$-D-galactopyranoside \\
\hline$\lambda$ & lambda / wavelength \\
\hline
\end{tabular}

\begin{tabular}{ll}
\hline Abbreviation & Meaning \\
\hline${ }^{\circ} \mathrm{C}$ & degree Celsius \\
$\mathrm{A}$ & ampere \\
$\mathrm{b}$ & base
\end{tabular}




\begin{tabular}{ll}
\hline Abbreviation & Meaning \\
\hline bar & bar (100 kPa) \\
bp & base pair \\
$\mathrm{g}$ & gram \\
$\mathrm{h}$ & hour \\
$\mathrm{Hz}$ & hertz (cycle per second) \\
$\mathrm{kb}$ & kilo base pair (103 bases) \\
$\mathrm{I}$ & liter \\
$\mathrm{M}$ & molar (mole/l) \\
min & minute \\
$\mathrm{rpm}$ & round per minute \\
sec & second \\
$\mathrm{U}$ & units \\
$\mathrm{V}$ & volt \\
$\mathrm{W}$ & watt \\
mole & number of atoms in $12 \mathrm{~g}$ carbon \\
$\mathrm{xg}$ & x g-forces \\
\hline
\end{tabular}

\begin{tabular}{lll}
\hline One letter code & Three letter code & Meaning \\
\hline A & Ala & alanine \\
C & Cys & cysteine \\
D & Asp & aspartic acid \\
E & Glu & glutamic acid \\
F & Phe & phenylalanine \\
G & Gly & glycine \\
H & His & histidine \\
I & He & isoleucine \\
K & Lys & lysine \\
L & Leu & leucine \\
M & Met & methionine \\
N & Asn & asparagine \\
P & Pro & proline \\
Q & Gln & glutamine \\
R & Arg & arginine \\
S & Ser & serine \\
T & Thr & threonine \\
Y & Thy & tyrosine \\
V & Val & valine \\
W & Trp & tryptophan \\
\hline
\end{tabular}

\begin{tabular}{llll}
\hline Abbreviation & Meaning & Abbreviation & Meaning \\
\hline $\mathrm{k}$ & kilo $\left(10^{3}\right)$ & $\mathrm{A}$ & adenine \\
$\mathrm{m}$ & milli $\left(10^{-3}\right)$ & $\mathrm{C}$ & cytosine \\
$\mu$ & micro $\left(10^{-6}\right)$ & $\mathrm{G}$ & guanine \\
$\mathrm{n}$ & nano $\left(10^{-9}\right)$ & $\mathrm{T}$ & thymine \\
$\mathrm{p}$ & pico $\left(10^{-12}\right)$ & $\mathrm{U}$ & uracil \\
\hline
\end{tabular}


Cyclic di-AMP and osmoregulation in Listeria monocytogenes 
Introduction

\section{Introduction}

The $17^{\text {th }}$ and $18^{\text {th }}$ centuries were a time full of new scientific discoveries, with observations that seemed rather unexplainable for the people of the era. Not only did Antonie van Leeuwenhoek observe microorganisms for the first time and thereby opened the door to a strange and unknown world, but also the first description of osmosis was recorded in this period (van Leeuwenhoek, 1677). The French physicist Jean-Antoine Nollet noticed that an alcohol filled cylinder that was sealed with a gallbladder increased in volume in a water bath (Nollet, 1748). The volume increased to such an extent that pressure built up and the alcohol would shoot out if the gallbladder is pricked with a needle. This is not only the first observation of osmosis, the directed flow of particles across-a semipermeable membrane, but also the first description of the resulting turgor pressure.

The next chapters will highlight the importance for bacteria to maintain a normal turgor pressure by osmoregulation, mechanisms they employ therefore and tools bacteria have developed to sense and regulate these mechanisms, including the second messenger c-di-AMP.

\section{Osmoregulation in bacteria}

Bacteria have to cope with various environmental conditions and their changes. Due to their confinement to an imminent location, bacteria have evolved a multitude of mechanisms to sense and cope with changes in environmental conditions, like the regulation of osmotic homeostasis (Sleator \& Hill, 2002; Wood, 1999). Osmotic homeostasis is the maintenance of a gradient of osmolytes across-the cell envelope. For bacteria and plants, which have a rigid cell wall, in contrast to animal cells, the difference between internal and external osmolarity is not isotone, but higher inside the cells. For Gram-negative bacteria, like Escherichia coli, an internal pressure (turgor) of about 0.33 bar has been reported, while for Gram-positive bacteria, like Bacillus subtilis, a pressure of about 10 bar has been reported (Rojas \& Huang, 2018). The osmotic pressure on a cell is determined by the concentration of osmolytes that are not able to diffuse through the cell membrane and therefore able to generate concentration gradients. Their ability to cross-the cell envelope is controlled by regulated transport mechanisms. An osmolyte is a substance that influences osmosis by its ability to bind water molecules; also called osmotic active substance. Prime examples are ions, amino acids, sugars and peptides and other charged molecules (Wood, 1999). Bacterial cells that live in dynamic environments have therefore evolved mechanism to cope with changes in external osmolarity - counteracting osmotic stress.

There are two kinds of osmotic stress: hypoosmotic stress, induced by a downshift (decrease) of external osmolytes and hyperosmotic stress, induced by an upshift (increase) of external osmolytes. During hypoosmotic stress, water follows the gradient of osmolytes and thereby increases the cellular volume, leading to a higher intracellular pressure and osmotic swelling. The opposite happens during hyperosmotic stress: cells release water, undergo a loss of turgor pressure, causing an invagination of the membrane. Bacteria have to cope with these two antagonistic stresses in different ways. To cope with a hypoosmotic stress it is important to prevent the additional import of osmolytes and to reestablish the natural concentration gradient by exporting osmolytes in the surrounding. Furthermore, mechanosensitive water channels, aquaporins, open rapidly and function as a kind of emergency pressure valve by releasing cytoplasmic content in the 
surrounding (Hoffmann et al., 2008) During hyperosmotic stress, the cell needs to import additional osmolytes and prevent secretion of cytosolic osmolytes to lower the concentration gradient between the high external and low internal osmolarity. The basic changes and reactions of bacterial cells to osmotic stress are illustrated in Fig. 1.1.

As reviewed by Jane M. Wood the effects of an osmotic upshift on structure and physiology of E. coli cells can be summarized in three main phases (Wood, 1999). An osmotic upshift leads to a rapid dehydration of the cell, accompanied with a shrinkage of the cytoplasmic water content, increased cytoplasmic crowding and altered strain on the cell envelope. These structural changes are accompanied by inhibition of respiration and many transport processes, activation of potassium and osmolyte import, a transient increase in $\mathrm{pH}$ difference and also a transient increase in the ATP concentration. After this initial period, which happens in the first one to two minutes, nucleic acid counterions are replaced and the cell rehydrates, potassium glutamate and compatible solutes accumulate, cell processes are resumed and $\mathrm{pH}$ and ATP levels normalize. This period takes up 20 to 60 minutes and is followed by the last phase that involves remodeling of the cell envelope and nucleoid, the resume of cellular functions and re-establishment of physiological gradients of osmolytes by expression of compatible solute import and/or export systems.

It is interesting to note that the first response bacteria employ to cope with a hyperosmotic stress usually is the rapid uptake of potassium ions (Kempf \& Bremer, 1998). The intracellular concentration of ions, including potassium, is crucial for the survival of cells. On the one hand it is necessary for proper ribosome and on the other hand to buffer the negative charge of the phosphate backbone of the DNA. Furthermore, it is essential for maintaining cellular turgor (Nierhaus, 2014; Pasi et al., 2015; Sleator \& Hill, 2002; Wood, 1999). Another osmolyte that is important in the first response is the negative charged amino acid glutamate. Glutamate is often designated as a counterion to potassium and its intracellular concentration, by synthesis and/or uptake, usually rises together with the potassium concentration (Yan et al., 1996). Since an excess of potassium would also be detrimental for the cell if exposed to too high concentrations or for a too long time, potassium is usually replaced by other low molecular weight compounds that can accumulate in the cell without disturbing cellular functions; also known as compatible solutes. Examples for compatible solutes are amino acids (e.g. proline or carnitine), oligopeptides, carbohydrates (e.g. trehalose) and other molecules such as glycine betaine (Wood et al., 2001). Maintaining cellular turgor is not only important for maintaining cellular shape and integrity, it is also a driving force of bacterial cell growth and an important factor influencing division (Rojas \& Huang, 2018). Moreover, it is not enough for the cells to accumulate osmolytes and compatible solutes, the cell also has to sense when the osmotic concentration gradients are again acceptable for the cell again and therefore when to stop synthesis or uptake of osmolytes.

The opposite case, a hypoosmotic shock, has to be counteracted by the cell in the opposite direction and can again be separated in three phases: the cell has to get rid of internal osmolytes and prevent the further influx of water molecules and osmotic swelling. In less than a minute after decreasing the external concentration of osmolytes, water flows into the cell, leading to a rapid hydration and swelling, a decrease in cytoplasmic crowding and also alterations of cell envelope strain. These effects are rapidly counteracted by the opening of mechanosensitive aquaporin channels that allow a rapid release of pressure by releasing cytoplasmic material and water molecules into the surrounding (Hoffmann et al., 2008). This first phase is followed by the shrinkage of the cell and a re-increase in cytoplasmic crowding and extrusion of water and co-solvents. In these few minutes, proton motive force probably also collapses. The effects in the third phase, 
which lasts only 10 to 20 minutes are mainly the closing of mechanosensitive channels, restoration of proton motive force and re-accumulation of osmolytes (Wood, 1999).

hyper-osmotic shock

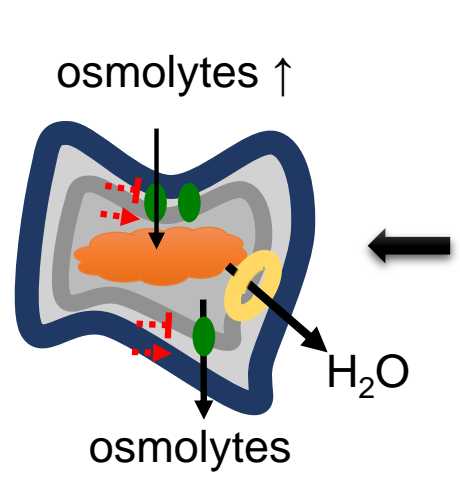

equilibrium

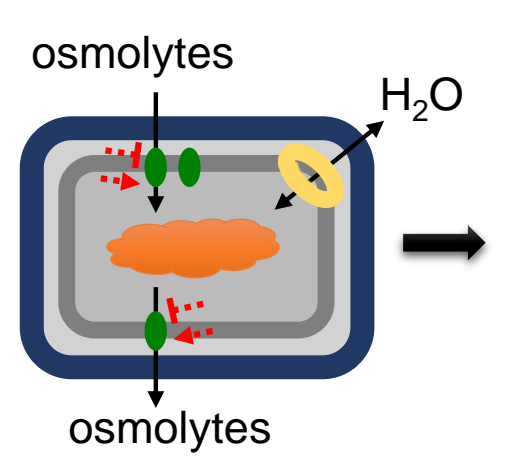

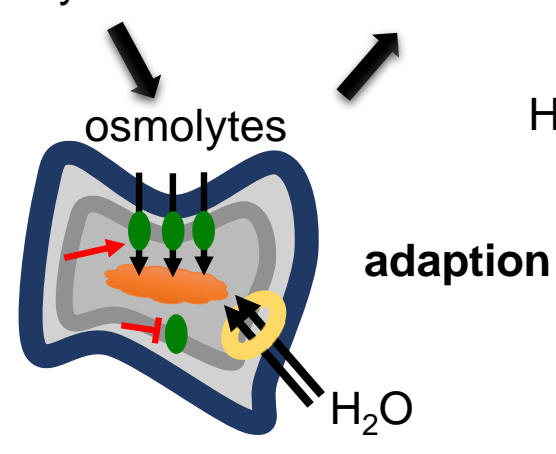

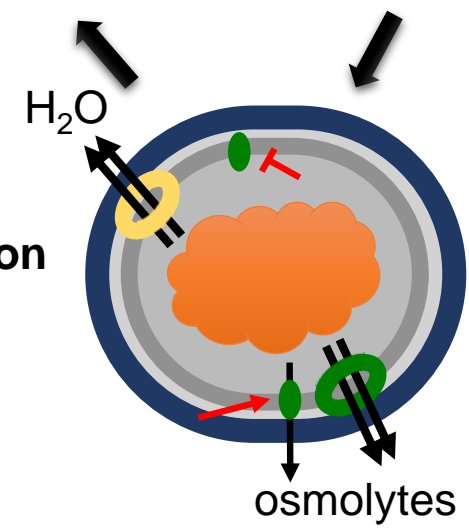

hypo-osmotic shock

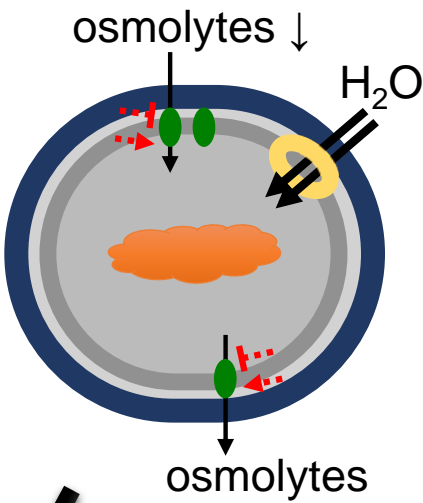

Fig. 1.1 Model depicting the effects of a hyper- and a hypoosmotic shock on bacterial cells and how they adapt. During a hyperosmotic shock, the external osmolyte concentration suddenly increases. This causes a rapid efflux of water, a shrinkage of the cellular volume and is accompanied by strain on the cell envelope. The cell adapts by increasing the intracellular osmolyte concentration through increased uptake and decreased export. There are secondary effects in this stage, e.g. shrinkage of the nucleoid due to influx of potassium ions and increased molecular crowding. In the end it promotes an influx of water and import and/or synthesis of compatible solutes that restore the equilibrium. The opposite, a hypoosmotic shock is triggered by a rapid decrease of external osmolytes. This leads to a rapid swelling of the cellular volume, accompanied by strain on the cell envelope. The cell adapts by decreasing the intracellular concentration of osmolytes by decreasing import and facilitating efflux through transporters and mechanosensitive channels. The decreased molecular crowding induces a de-condensation of the nucleoid and efflux of water. In the end the equilibrium is reconstituted. For further details see the text (modified from Commichau et al., 2018).

To summarize, a bacterial cell faces a variety of changes in cellular structure and physiology upon osmotic stress that have to be counteracted appropriately. It is therefore crucial for the cell to sense their internal and external osmotic state and to adjust accordingly to changes.

\section{Sensing of changes in osmolarity by bacteria}

As discussed above, the cell has to adjust to changes in extracellular osmolarity in an appropriate and timely manner. For this purpose, cells have evolved a variety of mechanisms to sense external and internal concentrations of osmolytes and changes in cellular turgor. One of those mechanisms that may be less straight forward are interactions between DNA and proteins. Despite its rather compact packaging, which reduces the space about four to ten times compared to free DNA (Martínez-Antonio et al., 2004), the nucleoid is estimated to have a DNA concentration of about 50- 
$100 \mathrm{\mu g} / \mu \mathrm{l}$ and an occupational space of about 12.5 to $20 \%$ of the total cytoplasmic lumen, which is highly dependent on the macromolecular crowding (Wood, 1999). The study by Gray et al. suggests a correlation between the nucleoid filled spaces and cell size that is independent of replication but species dependent; meaning that the nucleoid filled space increases with an increased cell size (Gray et al., 2018). For B. subtilis the average nucleoid area was reported to be to be around $3 \mu \mathrm{m}^{2}$ with an average cell area of $10 \mu \mathrm{m}^{2}$, resulting in a ratio of nucleoid to cell area of 0.3. E. coli on the other hand has an average nucleoid are of $2 \mu \mathrm{m}^{2}$, and an average cell area of $4.5 \mu \mathrm{m}^{2}$, resulting in a nucleoid to cell area ratio of about 0.45 . This demonstrates that proteinDNA interactions might have developed species specific, regarding protein amount and binding affinities (Gray et al., 2018). Their study also suggests no correlation between growth under different osmolarities and nucleoid to cell area ratio. However, transient effects that occur in a very short timeframe after an osmotic stress have not been investigated in this study. Other studies show a transient effect of osmotic stress on nucleoid size and structure (Cagliero \& Jin, 2013). Interestingly, osmotic stress seems to also alter the general packaging structure of DNA. Initial osmotic stress leads to an increase in supercoiling and the subsequent increased potassium transporter activity leads again to a relaxation (Meury \& Kohiyama, 1992). A DNA-based osmotic stress sensor therefore has to be adapted to the DNA concentration, the nucleoid to cellular area ratio and osmotic stress induced changes in cellular size; all of these alterations are species specific. Therefore, the expression and DNA-binding affinities as well as other factors like ion binding have evolved to allow a specific and timely response. Examples for osmotic-stress controlled DNA-binding are the RNA polymerase (RNAP) or the transcriptional regulator BusR. A study by Cagliero \& Jin demonstrates the rapid dissociation of the RNAP from the nucleoid after hyperosmotic stress. This is caused by condensation of the nucleoid due to increased molecular crowding after the initial import of potassium ions. Eventually, the RNAP re-associates again when osmoprotectants accumulate and the potassium concentration decreases (Cagliero \& Jin, 2013). BusR on the other hand is the transcriptional repressor of the busAA-busAB operon in Lactococcus lactis that encodes a glycine betaine importer (consisting of BusAA and BusAB). Romeo et al. found that BusR interaction with the complex of RNAP and the busA promoter is released in dependency of the ionic strength in vitro (Romeo et al., 2007). Therefore, after the initial osmotic stress phase, once the RNAP re-associates with the nucleoid and binds the busA promoter. The increase in ionic strength would prevent interaction of BusR, thereby allowing elevated expression of the BusA glycine betaine importer to import additional compatible solutes. This simplified model is of course not the complete story. In case of BusR additional regulatory mechanism have been identified, which are highlighted in the following chapters.

Interestingly, the most responsive systems allowing adaption to osmotic stress are localized in the plasma membrane. This is the case for both, potassium and osmolyte transporter that counteract an osmotic upshift and for mechanosensitive channels that counteract an osmotic downshift (Wood, 2011). One important determinant for transporter activity is the effect of solvents on the membrane. Similar as positive charged ions like magnesium, calcium, sodium or potassium mitigate the negative charge of the DNA backbone; ions also interact strongly with the charged headgroups of phospholipids, like phosphatidylglycerol or phosphatidylethanolamine (Roux \& Bloom, 1990). Alterations in surface charge due to an in- or decrease in local ion concentrations have therefore the power to impact protein-membrane and protein-protein interactions at the membrane. They can also affect protein activity and are therefore a potential signal that can be measured by the cell. More obvious than alterations in charge distributions, however, are the physical changes that osmotic up and downshifts pose on the lipid bilayer of cell membranes. Although the membrane is fluid, it is rather inelastic and strains through alteration of cellular turgor influence 
the lateral pressure on transmembrane (TM) domains of proteins and can thereby alter protein activity or lead to opening or closing of mechanosensitive channels (Sleator \& Hill, 2002; Wood, 2011). An interesting example for a mechanosensitive measuring system is the PhoQ/PhoP twocomponent system from E. coli. Interestingly, this system senses a whole variety of different stimuli, e.g. the extracellular magnesium concentration, low pH and the presence of antimicrobial peptides. Thereby it plays a crucial role for enterobacterial infection. In addition to those stimuli, it has also been shown that the system is able to sense an osmotic upshift by integrating the thickness and lateral pressure of the membrane on the TM helices of PhoQ. The conformational changes in the membrane are then passed on to the cytosolic kinase domain and thereby lead to an altered signal transduction (Yuan et al., 2017). Intriguingly, a variety of osmolyte transporters, such as OpuA from B. subtilis (glycine betaine transporter consisting of OpuAA-OpuAC), BetP from Corynebacterium glutamicum (glycine betaine) or ProP from E. coli (glycine betaine and proline) seem react to changes in osmolarity. Reconstitution experiments in proteoliposomes suggest that they react to changes in ion concentrations independent of changes in turgor (Poolman et al., 2004). However, the situation in vivo might be more complex and the transporter might integrate more than one stimulus and it is not yet fully understood if sensing is achieved by ligand binding, alterations in charges or ion distribution, hydration or changes in membrane strain, tension or curvature or a combination of them and might vary from transporter to transporter (Wood, 2011). One way the cell can integrate such a variety of different stimuli in a unified cell-wide answer is the use of second messenger signaling pathways. In those an environmental stimulus, such as changes in osmolarity, is sensed by different mechanisms and subsequently changes the concentration of a second messenger by altering synthesis, degradation and/or export. The osmolyte transporter of OpuCA of Listeria monocytogenes and Staphylococcus aureus, which are homologs of the above discussed OpuAA from $B$. subtilis have recently been identified to bind and be inhibited by the nucleotide second messenger cyclic di-AMP (c-di-AMP) that plays a major role in osmoregulation of Firmicutes (Huynh et al., 2016; Schuster et al., 2016).

\section{The nucleotide second messenger c-di-AMP}

Bacteria sense a multitude of different environmental changes with the corresponding signals sensed outside, inside the cell, in the cell envelope, through communication with other bacteria and many more. The bacteria have to integrate all these signals and respond appropriately. In this sensing process, so called, second messenger molecules play an important role. They function as an integrator, which controls biological processes upon changes in concentration. Signal input can modulate free second messenger concentration by synthesis, degradation, export or binding to target structures. Second messengers are furthermore known to bind and modulate very different target structures, such as RNAs or proteins (Newton et al., 2016).

Nucleotide second messenger have now been known since the discovery of cyclic adenosine monophosphate (CAMP) by the later Nobel laureate Earl Wilbur Sutherland, Jr. and colleagues in the 1950's (Rall et al., 1957; Rall \& Sutherland 1958). cAMP plays an important role in eukaryotes and in prokaryotes, ranging from regulation of sugar metabolism, over gene expression, to neuronal functions; highlighting the importance and diversity of second messenger signaling (Newton et al., 2016). Since the discovery of cAMP, a variety of nucleotide second messenger molecules has been identified and studied: cyclic di-guanosine monophosphate (c-di-GMP), cyclic guanosine monophosphate (cGMP), guanosine tetra- and pentaphosphate; ((p)ppGpp), cyclic guanosine 
monophosphate-adenosine monophosphate (cGAMP) or cyclic di-adenosine monophosphate (c-di-AMP) (Corrigan \& Gründling, 2013; Gomelsky, 2011; Kalia et al., 2013; Wu et al., 2012).

c-di-AMP is a quiet special nucleotide second messenger: it is the only known one that is essential in many c-di-AMP synthesizing bacteria, it is produced by many pathogenic Gram-positive bacteria, it is absent in humans and it is the first discovered second messenger that governs a biological process on two distinct levels - Regulation of gene expression and protein activity. For all these reasons is c-di-AMP a prime target for the development of novel antibiotics, which are desperately needed in a time of increased multiple antibiotic resistance in many clinically relevant Gram-positive bacteria, like S. aureus or Streptococcus pneumoniae (Laxminarayan et al., 2013; Rosenberg et al., 2015; WHO, 2014). c-di-AMP, together with two molecules of pyrophosphate ( $\left.\mathrm{PP}_{\mathrm{i}}\right)$, is synthesized by the diadenylate cyclase (DAC) domain containing proteins from two molecules of adenosine triphosphate (ATP). The synthesis of c-di-AMP, however, has also been reported from one molecule of ATP and one molecule of adenosine diphosphate (ADP) in Mycobacterium tuberculosis (Manikandan et al., 2014; Witte et al., 2008). Degradation of c-di-AMP is facilitated by specific phosphodiesterases (PDEs) into the linear phosphoadenylyl adenosine ( $\mathrm{pApA}$ ) that can be further hydrolyzed to AMP. The known PDEs contain either DHH/DHHA1 or HD domains, like PdeA (GdpP in B. subtilis) or PgpH, respectively (Huynh et al., 2015; Rao et al., 2010).

\section{Synthesis and degradation of c-di-AMP}

While many pathogenic firmicutes, like L. monocytogenes, S. aureus or S. pneumoniae only harbor one DAC domain containing enzyme of the CdaA/DacA-type, B. subtilis contains three different type of DACs: the DNA-binding protein DisA, the membrane bound CdaA and the sporulation specific CdaS. Interestingly, B. subtilis is able to survive if only one cyclase is expressed, highlighting that c-di-AMP is essential independent of the producing enzyme (Mehne et al., 2013). The DisAtype (DNA integrity scanning protein A) of DAC is present in spore-forming firmicutes, like Clostridium difficile, in actinobacteria, like C. glutamicum, in M. tuberculosis, in the hyperthermophilic bacterium Thermotoga maritima and has even been described in archaea, like Methanocaldococcus jannaschii (Bai et al., 2012; Commichau et al., 2019; Kellenberger et al., 2015; Witte et al., 2008). DisA, which forms homooctamers, has been shown to bind DNA, moving along it and being stalled upon DNA damages that lead to an inhibition of cyclase activity. Furthermore, it interacts with other proteins of DNA repair pathways such as the branch migration transferase RadA (Oppenheimer-Shaanan et al., 2011; Witte et al., 2008; Zhang \& He, 2013). The function and regulation of the CdaS-type (sporulation-specific cyclic di-AMP synthase) DAC is less well studied. It is known to play a role in sporulation initiation and/or efficient germination and that it forms hexamers that consists of three homodimers by interaction of the two N-terminal domains of CdaS, which if truncated lead to hyperactivity (Mehne et al., 2014; Zheng et al., 2015). The most widespread kind of DAC is of the CdaA-type (cyclic di-AMP synthase A). CdaA, which is also called DacA (Corrigan et al., 2011; Mehne et al., 2013; Rismondo et al., 2016; Rosenberg et al., 2015; Woodward et al., 2010), is a membrane bound protein. It consists of three $\mathrm{N}$-terminal TM domains and the DAC domain, which is flanked by two coiled-coil domains. The gene encoding for CdaA is conserved together with the cdaR gene, encoding a protein of unknown function and the gene encoding the essential glucosamine mutase GImM. In $B$. subtilis they are encoded in one operon, while in L. monocytogenes for example $c d a A$ and $c d a R$ are in one operon followed by $g / m M$ with its own promoter (Mehne et al., 2013; Rismondo et al., 2016). CdaA is active in vitro with the metal ions $\mathrm{Mn}^{2+}$ or $\mathrm{Co}^{2+}$, in contrast to DisA, which is active with $\mathrm{Mg}^{2+}$ as a cofactor. It furthermore forms 
homodimers, which are important for its activity. Depletion of CdaA and thereby c-di-AMP has been linked to alterations in the cell wall metabolism and increased susceptibility to cell wall-acting antibiotics (Rismondo et al., 2016; Witte et al., 2013). The CdaR protein consists of an N-terminal TM domain and four YbbR domains of unknown function. It has been demonstrated to selfinteract via the YbbR domains and to interact only in its full-length variant with the full-length CdaA protein. Moreover, a deletion of $c d a R$ leads to altered c-di-AMP levels in B. subtilis, L. monocytogenes and $S$. aureus and is able to influence $C$ daA activity if co-expressed in $E$. coli, suggesting that CdaR is a modulator of CdaA activity (Bowman et al., 2016; Gundlach et al., 2015b; Mehne et al., 2013; Rismondo et al., 2016). Although protein crystallographic structures of the YbbR domains are available, which show some structural homology to domains of unknown function from L25 and TL5 ribosomal binding protein, their function as putative sensory domains is not yet elucidated (Barb et al., 2010). The second protein, which has been shown to play a role in regulating CdaA activity is GImM. GImM converts glucosamine-6-phosphate to glucosamine-1-phosphate, which is one of the first reactions for the synthesis of $\mathrm{N}$-acetylglucosamine, an important building block of the bacterial peptidoglycan (Barreteau et al., 2008; Mengin-Lecreulx \& van Heijenoort, 1996). GImM has been shown to interact with CdaA in B. subtilis and is hypothesized to affect its activity (Gundlach et al., 2015b). Moreover, in L. lactis an osmoresistant suppressor mutant with a mutation in GImM was identified that lead to a decrease in the intracellular c-di-AMP concentration, demonstrating a functional link between GImM and CdaA activity (Zhu et al., 2016). Intriguingly, today a novel study was published by the Gründling lab, demonstrating an inhibitory effect of GImM on CdaA activity in S. aureus in vitro and in vivo. They propose a model, were interaction of GImM with the DAC domain perturbs CdaA self-interaction and therefore catalytic activity (Tosi et al., 2019).

c-di-AMP has just been identified about 10 years ago and as shown above, novel discoveries and hypothesis are very frequent. Another recent example is the discovery of a founding member of a novel DAC class. In the minimal and cell wall-less human pathogen Mycoplasma pneumoniae an enzyme that produces c-di-AMP was discovered, designated CdaM, while both CdaR and GImM homologs are not present in this organism (Blötz et al., 2017). Recent discoveries, furthermore, demonstrate the presence of even more classes of DAC domain containing enzymes in different organisms, reviewed elsewhere (Commichau et al., 2019).

Additionally, another important part of the c-di-AMP metabolism is to regulate the concentration by degradation or export. In studies with L. monocytogenes, multidrug resistance transporters (Mdr) where identified to export c-di-AMP. As a consequence, c-di-AMP triggers a type I interferon response in human cells by activation of the innate immune sensor STING. Furthermore, it also leads to inhibition of the oxidoreductase RECON, resulting in anti-inflammatory responses and intriguingly increased cell-to-cell spread of L. monocytogenes, highlighting c-di-AMPs effect in virulence (Archer et al., 2014; Kaplan Zeevi et al., 2013; McFarland et al., 2017; McFarland et al., 2018). Efflux of c-di-AMP may also be a fast way to decrease the intracellular concentration independent of degradation. Degradation occurs via hydrolyzation by c-di-AMP-specific PDEs. In B. subtilis and L. monocytogenes two different PDEs are synthesized, GdpP (PdeA in L. monocytogenes) and PgpH. GdpP is of the DHH/DHHA1 type an consists of two TM domains, followed by a PAS, a GGDEF domain and the catalytic DHH/DHHA1 domain. GdpP is competitive inhibited by ppGpp another second messenger that is important in regulating the stringent response during nutrient starvation, suggesting cross-talk between the c-di-AMP and ppGpp regulatory networks (Rao et al., 2010). Furthermore, the PAS domain of GdpP has been shown to inhibit catalytic activity of GdpP upon binding of heme, suggesting a link between c-di-AMP and the redox state of the cell (Rao et al., 2011). PgpH, a member of the second major class of c-di-AMP hydrolyzing 
enzymes consists of an extracellular 7TMR HDED domain, followed by seven TM helices and the catalytic HD domain. As for GdpP, PgpH was also found to be inhibited by ppGpp, again suggesting a cross-talk between c-di-AMP signaling and the stringent response (Huynh et al., 2015). PgpH was furthermore identified to be the major c-di-AMP degrading enzyme in B. subtilis, with its deletion leading to an accumulation of c-di-AMP. Interestingly, a gdpP pgpH double mutant lead to toxic accumulation, which is why c-di-AMP is also termed "essential poison", highlighting the importance to maintain adequate levels of this second messenger (Gundlach et al., 2015b). Accumulation of c-di-AMP by PDE deletion was furthermore found to deregulate expression of about 700 genes and in consequence lead to defects in biofilm formation and plant attachment of $B$. subtilis (Gundlach et al., 2016; Townsley et al., 2018). There are also other classes of PDEs, degrading c-di-AMP to PApA and further to AMP, nano-RNases that degrade PApA to AMP or PDEs that degrade c-di-AMP extracellular, which are covered in a recent review by Commichau and colleagues (Commichau et al., 2019).

The study by Gundlach and colleagues also demonstrates why the essential function of c-di-AMP has eluded discovery for almost a decade (Gundlach et al., 2016): the plethora of different phenotypes and intertwines of different signaling pathway, ranging from cell wall phenotypes, over lifestyle changes, DNA damage responses to the discovery of c-di-AMPs involvement in osmoregulation (Corrigan et al., 2011; Commichau et al., 2015; Dengler et al., 2013; Gundlach et al., 2017; Luo \& Helmann, 2012; Rismondo et al., 2016; Whiteley et al., 2015; Whiteley et al., 2017; Witte et al., 2013).

\section{The signaling network of c-di-AMP}

The first identified bacterial c-di-AMP-regulated protein was the TetR-like transcriptional factor DarR in Mycobacterium smegmatis (Zhang et al., 2013). Binding of c-di-AMP has been shown to stimulate DNA-binding of DarR that in consequence negatively regulates expression of an operon coding for three genes, including a major facilitator family transporter, a medium chain fatty acylCoA ligase and a homolog of the cold shock protein CspA. Since this study, c-di-AMP was found to regulate a variety of cellular targets in bacteria, of, which many are highlighted in Fig. 1.2. c-di-AMP was identified to bind and regulate the potassium import system KtrAB by binding to the RCK_C domain of the cytosolic KtrA and cation/proton antiporter CpaA in S. aureus that putatively functions as a potassium export system, which were also subjects of further studies illuminating the molecular mechanisms (Chin et al., 2015; Corrigan et al., 2013; Kim et al., 2015). Interestingly, c-di-AMP would thereby control potassium homeostasis in S. aureus by inhibiting import and stimulating export. In the same study, the PII-like protein PstA of unknown function and the histidine kinase KdpD of the KdpDE two component system were identified as c-di-AMP binding proteins (Corrigan et al., 2013). While the function of PstA is unknown, KdpDE regulated expression of the $\mathrm{Kdp}(\mathrm{F}) \mathrm{ABC}$ potassium import system, indicating again an important role for c-di-AMP in potassium homeostasis. Intriguingly, translation of KtrAB and $\mathrm{YdaO}$ (which was later identified as a high-affinity potassium importer in $B$. subtilis and renamed $\operatorname{KimA}$ ) are regulated by a c-di-AMP responsive riboswitch. Binding of c-di-AMP to the $y d a O(\operatorname{kimA})$ riboswitch prevents translation of the potassium importers. This makes c-di-AMP a special regulator and the first of its kind by regulating a biological process by controlling synthesis and activity (Gao \& Serganov, 2014; Gundlach et al., 2017; Moscoso et al., 2015; Nelson et al., 2013). 


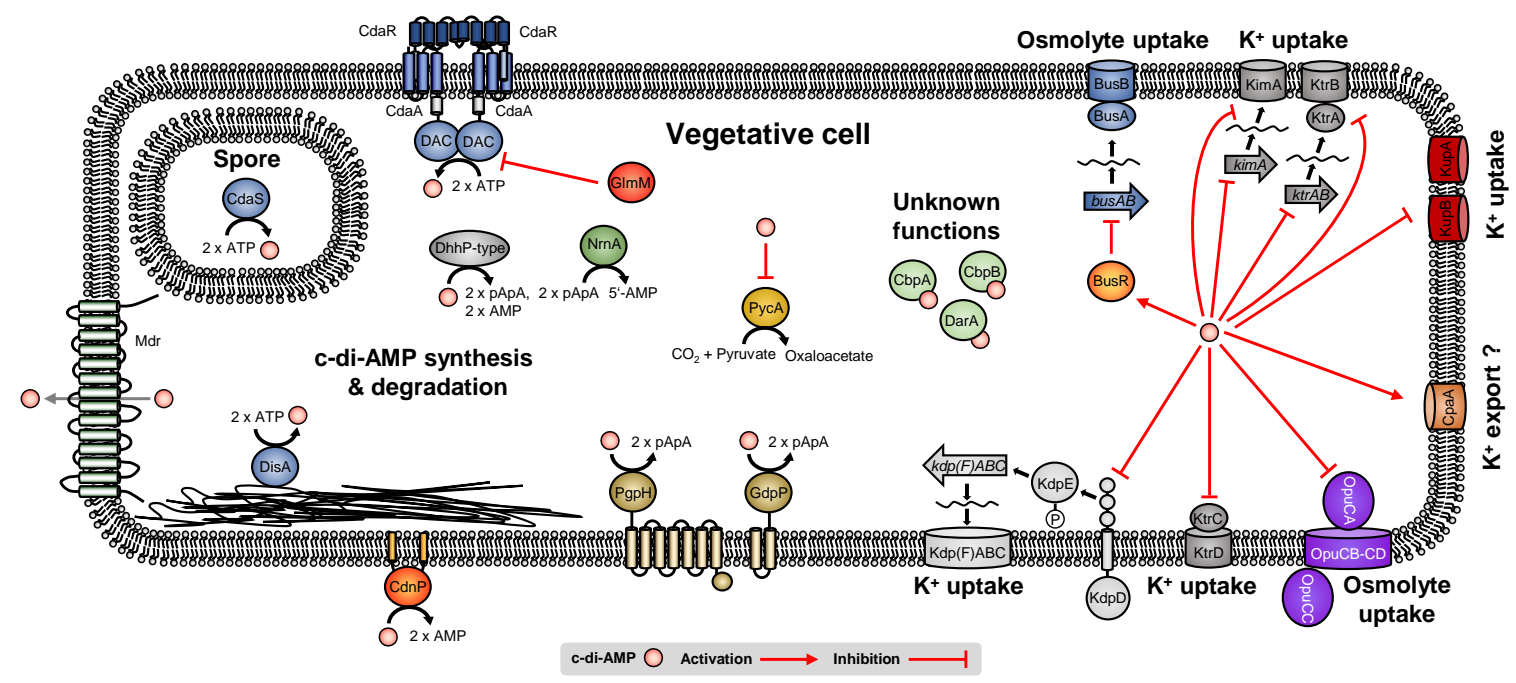

Fig. 1.2 Synthesis and degradation of the essential second messenger c-di-AMP and its cellular targets. c-di-AMP is synthesized by DACs. Shown are members of the main three types of DACs: CdaA, DisA and CdaS. CdaA activity is modulated by the YbbR-domain containing protein $\mathrm{CdaR}$ by the cell wall precursor synthesizing glucosamine mutase GImM. c-di-AMP is degraded by PDEs to PApA. Shown are members of the main types of PDEs, GdpP and PgpH and also of the DhhP- (that can also degrade pApA further to AMP) and the CdnP-type, (that degrades c-di-AMP to AMP) as well as the pApA to AMP degrading nano-RNase NrnA. Shown is also MdrT, a member of c-di-AMP secreting multidrug resistance transporters. Furthermore, targets that have been shown to bind c-di-AMP are depicted: the pyruvate carboxylase PycA, which is allosterically inhibited by c-di-AMP and the proteins of unknown function: $\operatorname{DarA}, \mathrm{CbpA}$ and $\mathrm{CbpB}$ of $L$. monocytogenes. Moreover, the known c-di-AMP-controlled osmolyte transporters are shown: The high-affinity potassium uptake systems KtrAB and KimA that are controlled on protein level and via a c-di-AMP-binding riboswitch in B. subtilis, the $\mathrm{Kdp}(\mathrm{F}) \mathrm{ABC}$ high-affinity potassium transporter, whose transcription is regulated via the c-di-AMP inhibited two component system KdpDE in $S$. aureus and the low-affinity potassium transporter KtrCD. Two novel high-affinity potassium transporters, KupA and KupB, which were recently identified as c-di-AMP regulated in L. lactis are depicted, as well. c-di-AMP, moreover, regulates the putative potassium exporter $\mathrm{CpaA}$ in $\mathrm{S}$. aureus and binds the BusR transcription factor that inhibits expression of the glycine betaine importer BusAB in L. lactis and S. agalactiae. Finally, the OpuC glycine betaine transporter from B. subtilis is depicted, too (modified from Commichau et al., 2018).

To emphasize the importance of c-di-AMP on potassium homeostasis, it should be noted that it was identified to regulate potassium import in a whole variety of different bacteria. It was found to inhibit uptake in S. pneumoniae via binding to the CabP protein that interacts with the potassium importer TrkH and thereby inhibits import (Bai et al., 2014). Interestingly, a later study showed that CabP deletion leads to reduced c-di-AMP concentrations in S. pneumoniae, indicating that either CabP or the resulting altered potassium homeostasis is able to be senses and thereby to modulate c-di-AMP homeostasis (Zarrella et al., 2018). Moreover, c-di-AMP concentrations were recently identified to be light-dependent in the cyanobacterium Synechococcus elongatus and a cdaA deletion mutant was more susceptible to oxidative stress during the nighttime part of a day-night cycle. Interestingly, S. elongatus encodes several putative c-di-AMP regulated potassium importers and an impairment in potassium transport leads to a similar susceptibility to oxidative stress, as it was shown for the cdaA mutant (Rubin et al., 2018). In L. lactis, c-di-AMP has been shown to inhibit potassium uptake via KupA and KupB of the Kup family potassium importer and even in the minimal organism M. pneumoniae, c-di-AMP was identified as a regulator of potassium uptake (Blötz et al., 2017; Quintana et al., 2019). Eventually, c-di-AMP was shown to be essential due to its central role in regulating potassium homeostasis in B. subtilis (Gundlach et al.,2017). Taken together this demonstrates the critical role of c-di-AMP in potassium homeostasis in a broad spectrum of different bacteria.

c-di-AMP was also found to regulate other cellular processes or bind to proteins whose function is not yet elucidated. It was shown to allosterically inhibit the pyruvate carboxylase PycA in 
L. monocytogenes and L. lactis or bind to the S. aureus PstA homologs in B. subtilis (DarA) or L. monocytogenes (PstA). The same is also true for two other proteins of unknown function, CbpA and $\mathrm{CbpB}$ in $\mathrm{L}$. monocytogenes and $\mathrm{NrdR}$, the negative regulator of the ribonucleotide reductase operon nrd (Campeotto et al., 2015; Choi et al., 2015; Choi et al., 2017; Gundlach et al., 2015a; Müller et al., 2015a; Sureka et al., 2014). While the function of PstA/DarA or the c-di-AMP binding proteins $\mathrm{CbpA}$ or $\mathrm{CbpB}$ is not yet understood, recent studies identifying novel binding proteins or genetic linkages via c-di-AMP dependent suppressor mutations demonstrate that c-di-AMP is not only regulating potassium homeostasis, but homeostasis of osmolytes in general. c-di-AMP can therefore be described as a major regulator for osmoregulation in Firmicutes. Whiteley et al. identified the growth of $L$. monocytogenes in a defined minimal medium as the first reported condition where c-di-AMP was non-essential (Whiteley et al., 2015). They could show that the limitation of osmolytes allows deletion of $c d a A$ and that accumulation of suppressor mutations in genes encoding oligopeptide uptake systems (OppABCDF), the PII-like protein PstA, the alarmone synthase/hydrolase RelA, a glycine betaine importer (GbuABC), the pyruvate carboxylase, the potassium importer homolog Lmo0993 (KtrD) or the CbpB protein contributed, among others, to suppression of c-di-AMP essentiality on complex media. In their second study they could, furthermore, show that the mutations in the opp and gbu genes are sufficient to allow $c d a A$ deletion mutants to grow on complex medium, resulting in the hypothesis that uncontrolled accumulations of oligopeptides and glycine betaine under these conditions is detrimental for growth of L. monocytogenes. Interestingly, they could further show that c-di-AMP impacts regulation of the flux in the citric acid cycle, an import cross-section between carbon and nitrogen metabolism and therefore also biosynthesis of amino acids that also act as osmolytes (Whiteley et al., 2017). c-di-AMP, furthermore, has been identified to regulate uptake of the osmolyte carnitine in L. monocytogenes by binding to the CBS domain of OpuCA of the OpuC transporter but not to the CBS domain in GbuA of the GbuABC glycine betaine importer or the CBS domain of BilEA, of the bile salt specific exporter BilE (Huynh, et al., 2016). Binding of c-di-AMP to OpuCA was also confirmed for S. aureus but again other CBS domain containing proteins, including the magnesium transporter MgtE and the transcription factor $\mathrm{CcpN}$, where not identified as c-di-AMP binding proteins (Schuster et al., 2016). Interestingly, although c-di-AMP has been established as a major determinant for osmotic regulation in S. aureus, due to its effect on osmolyte (glycine betaine via OpuD) and amino acid uptake (AlsT), it also seems to have a great impact on cell respiration and is dispensable under anaerobic conditions in this bacterium, highlighting that the complex regulatory effects of c-di-AMP are even with the increased knowledge not yet fully understood (Zeden et al., 2018). c-di-AMP has furthermore been identified to regulate osmotic homeostasis in Streptococcus agalactiae and L. lactis, by binding to BusR, that upon binding negatively regulates expression of the genes encoding BusAB, a glycine betaine importer (Devaux et al., 2018; Pham et al., 2018). Interestingly, in the study of Pham and colleagues, impact of osmotic up- and downshifts on c-di-AMP concentrations in different bacteria and mutants was investigated, too. They could show, that upon osmotic upshift (hyperosmotic shock) c-di-AMP levels rapidly decrease and vice versa increase upon hypoosmotic shock. Intriguingly, energizing of the cells that is required for ATP synthesis and presence of the DAC CdaA and the PDE GdpP/PdeA seemed to have the greatest influence on the bacteria to adapt their c-di-AMP concentration accordingly (Pham et al., 2018). Taken together, c-di-AMP has been shown to influence many cellular processes, predominantly osmotic homeostasis, in many bacteria that produce this nucleotide second messenger, with L. monocytogenes being a prominent bacterium of c-di-AMP research (Commichau et al., 2015; Commichau et al., 2018). 
Introduction

\section{Listeria monocytogenes and c-di-AMP}

The bacterium L. monocytogenes has been the subject of many c-di-AMP related studies: the first crystal structure of a CdaA-type DAC, the most abundant one, was obtained from a truncated L. monocytogenes enzyme (Rosenberg et al., 2015), many c-di-AMP binding proteins, such as CbpA, CbpB, PycA or OpuCA were first identified in this bacterium (Huynh et al., 2016; Sureka et al., 2017). Moreover, L. monocytogenes was the first bacterium were conditions were identified under which c-di-AMP is dispensable (Whiteley et al., 2015).

L. monocytogenes is closely related to $B$. subtilis, belonging also to the phylum of the Firmicutes. It was first described in 1926 by Everitt George Dunne Murray as Bacterium monocytogenes and later on named in honor of the British surgeon Joseph Lister and the upon infection occurring monocytosis (Gray \& Killinger, 1966; Murray et al., 1926; Pirie, 1940). One interesting aspect of L. monocytogenes is its lifestyle change. On the one hand, the bacterium can be found ubiquitous in nature as a saprophyte and on the other hand it is a facultative foodborne human pathogen (Farber \& Peterkin, 1991; Freitag et al., 2009). L. monocytogenes therefore needs to precisely sense its environment to adapt accordingly. Here, the transcription factor PrfA (positive regulator of listeriolysin A), the master regulator of the virulence of L. monocytogenes is the key player. Upon changes in temperature, nutrient conditions, components of stress response pathways, like the metabolic regulator CodY and effector molecules, such as glutathione, amount and activity state of PrfA is altered (de las Heras et al., 2011; Lobel et al., 2015; Reniere et al., 2015). In turn, PrfA-dependent genes that are important for triggering endocytosis (esp. internalins InIA and InIB), intracellular vacuolar escape (specific lipases PC-PLC, PI-PLC and the lytic enzyme LLO), uptake of nutrients (hexosephosphate transporter $\mathrm{Hpt}$ ), intracellular traversation (actin polymerization by ActA) and infection of neighboring cells are expressed (Chico-Calero et al., 2002; Scortti et al., 2007). Another key feature of $L$. monocytogenes is its ability to actively cross-protection barriers inside humans, such as the blood-brain or blood-placenta barrier, making it a dangerous bug for immunocompromised persons with the possibility of high mortality rates after encephalitis or meningitis and for pregnant women with the danger of prenatal infections, leading to severe consequences for the fetus, including disabilities, stillbirths or miscarriages (Low and Donachie, 1997; Vázquez-boland et al., 2001).

The adaptation of $L$. monocytogenes to such different environmental niches makes it a very interesting candidate to study osmohomeostasis. The bacteria must on the one hand be able to withstand changes in solute concentrations by environmental events such as changes in humidity and on the other hand be able to survive the transition to the intracellular life style, featuring passage through the stomach, exposure to bile salts in the gut and transition from a natrium rich to a potassium rich environment. One big difference between $B$. subtilis and $L$. monocytogenes is the inability of $L$. monocytogenes to form spores as a persistence state that is resistance against many environmental stresses and its ability despite that to be resistant against various $\mathrm{pH}$, temperature or salt stresses (Farber \& Peterkin, 1991). The main strategy for L. monocytogenes is hereby the accumulation of the compatible solutes glycine betaine, carnitine, glutamate and oligopeptides with some indications that glycine betaine uptake is also triggered upon cold stress in L. monocytogenes (Ko et al., 1994; Smith, 1996; Whiteley et al., 2017). Interestingly, glycine betaine is cotransported with sodium ions, which in turn have to be exported by putative cation-proton antiporter systems, leading to an increase in internal protons that in turn could be exported again by electron transport system, thereby linking osmolyte uptake to generation of proton motive force that has also been linked to c-di-AMP itself (Wood, et al., 2001; Zeden et al., 2018). Fig. 1.3 shows an overview of the most prominent systems or putative homologs of $L$. monocytogenes to known 
systems of B. subtilis (MrpA-G, NhaK, KimA, KtrCD, MscL, MscS and NrnA) or S. aureus (KdpFABCDE) that are involved in osmoregulation and/or c-di-AMP metabolism.
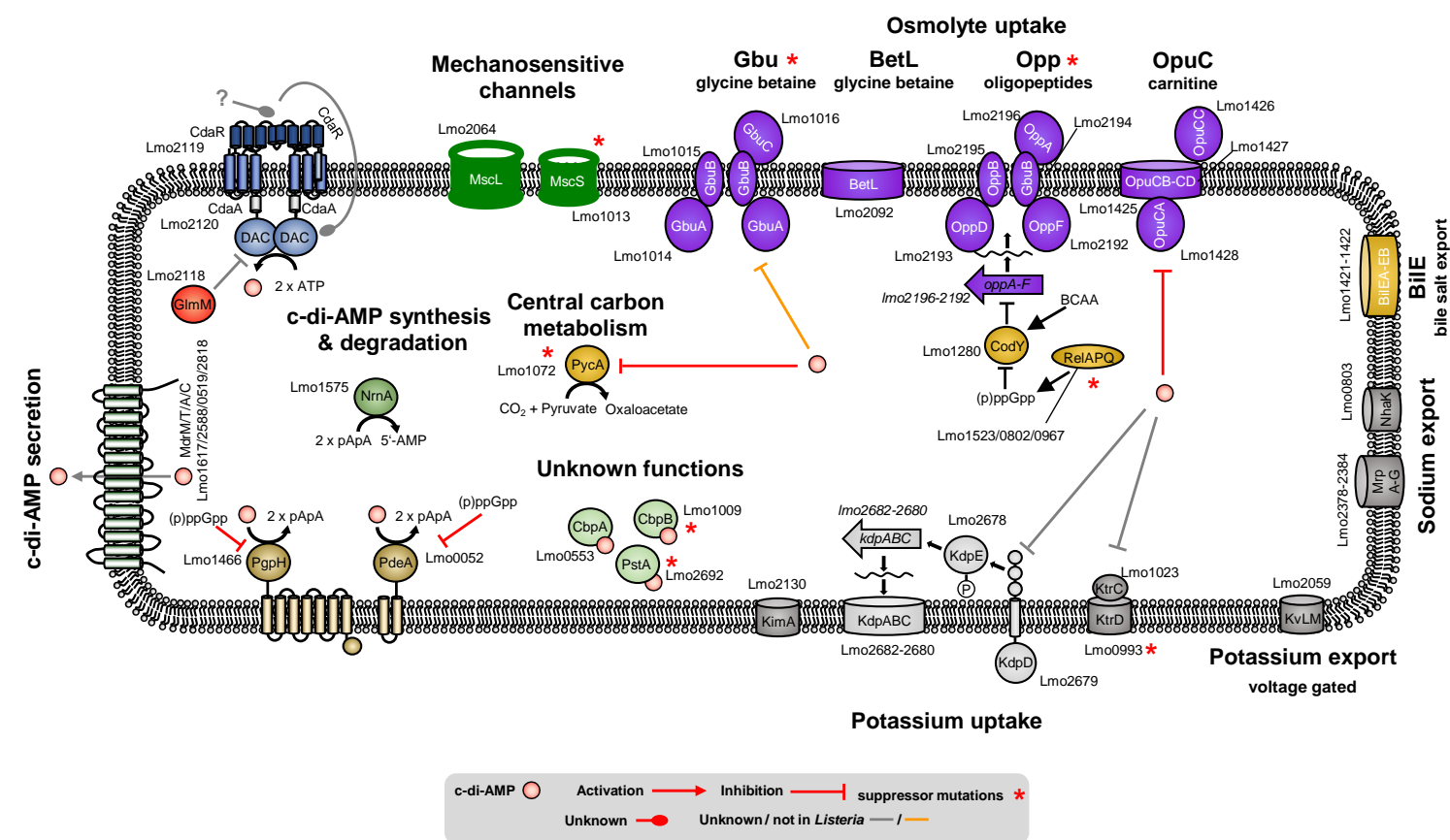

Fig. 1.3 c-di-AMP signaling and osmoregulation in L. monocytogenes. c-di-AMP is synthesized in L. monocytogenes by the sole DAC CdaA that is regulated by CdaR and GImM in L. monocytogenes. c-di-AMP has been shown to be secreted by the multidrug resistance transporters and degraded by the PDE PdeA (GdpP) and PgpH. L. monocytogenes also harbors a homolog of the nano-RNase NrnA that has been shown to degrade pApA further to AMP. c-di-AMP binds to the proteins of unknown function $\mathrm{CbpA}, \mathrm{CbpB}$ and $\mathrm{Pst} A(\mathrm{Dar} A)$. It has also been shown to inhibit the pyruvate carboxylase PycA and the osmolyte (carnitine) uptake system OpuC in L. monocytogenes. c-di-AMP has also been shown to inhibit uptake of glycine betaine in other organisms via homologs of the Gbu osmolyte transporter, but no binding could be identified for L. monocytogenes. In other bacteria, c-di-AMP has been shown to inhibit potassium uptake of the KtrCD low affinity potassium transporter and to inhibit transcription of the KdpABC high affinity transporter by binding to $\mathrm{KdpD}$ of the KdpDE two-component system. Despite those c-di-AMP-related systems, L. monocytogenes possesses other proteins that have been shown to play a role in un- or specific osmoregulation. Those are the mechanosensitive channels MscL and MscS (large and small conductance, respectively), the glycine betaine uptake system BetL, the voltage gated potassium exporter Lmo2059 (KvLm), homologs of the B. subtilis sodium exporter NhaK and MrpA-G. L. monocytogenes also expressed a bile salt specific exporter that is important in virulence (BilE) and an oligopeptide transporter, whose expression is regulated by CodY and the stringent response. In L. monocytogenes C-di-AMP signaling related suppressor mutations have been found in $\mathrm{CbpB}$, PstA, KtrD, RelA, Gbu system, Opp system and PycA, indicating a functional relationship between them and c-di-AMP signaling (based on Commichau et al., 2018).

As depicted, L. monocytogenes encodes homologs of two high-affinity potassium uptake systems, KimA and KdpABC, that are translational or transcriptional controlled by c-di-AMP in B. subtilis and $S$. aureus respectively. Interestingly, the kimA riboswitch seems to be absent in L. monocytogenes (Nelson et al., 2013). L. monocytogenes also encodes a voltage gated potassium transporter KvLm (Lmo2059; Santos et al., 2008; Santos et al., 2012) and homologs of two potential sodium export system Lmo0803 (NhaK) and Lmo2378-2374 (MrpA-G). Furthermore, L. monocytogenes possesses two uptake system for glycine betaine, GbuABC and BetL, an uptake system for oligopeptides (OppABCDF), an uptake system for carnitine (OpuC) and a bile salt specific exporter (BilE), demonstrating a broad set of different osmolyte transport systems (for further details see chapters above). Of those Gbu, Opp and Opu have been directly or indirectly implicated to be regulated by c-di-AMP (Fig. 1.3). Genes for homologs of two mechanosensitive channels Lmo2064 and Lmo1013 (MscL and MscS, respectively) and the c-di-AMP binding proteins, PycA, CbpA, CbpB and PstA (DarA) are also present. Several c-di-AMP secreting multi drug efflux systems (MdrMTAC) and two PDEs PdeA (GdpP) and PgpH and a potential homolog of the nano-RNase NrnA (Lmo1575) are 
present, as well. Concerning the synthesis of c-di-AMP, L. monocytogenes harbors only on DAC domain containing enzyme, $\mathrm{CdaA}$, or also called DacA, encoded together with its regulator CdaR and also the GImM enzyme (Commichau et al., 2018; Kaplan Zeevi et al., 2013; Rismondo et al., 2016; Rosenberg et al., 2015; Sureka et al., 2014; Whiteley et al., 2015; Whiteley et al., 2017; Woodward et al., 2010).

L. monocytogenes has a broad set-up of osmolyte transport systems, interesting life-style changes and is known for its adaptiveness. The bacterium encodes only one DAC, of the most abundant CdaA-type that is a prime target for finding novel antibiotics. These characteristics make L. monocytogenes an interesting subject to study c-di-AMP and its effect on osmoregulation and especially how synthesis of c-di-AMP might be regulated and coupled to sensing alterations in the osmotic state of the environment. 


\section{Objective of this thesis}

One of the major open questions about c-di-AMP signaling is how the cell senses changes in osmolarity to modulate DAC activity. The DAC CdaA is the most abundant type of c-di-AMP synthesizing enzyme, present in a lot of firmicute pathogens and hence an interesting target for novel antibiotics. It was hypothesized that CdaA activity is modulated by the CdaR and the GImM protein. The YbbR domains of CdaR have an unknown function and are a potential sensory domain that allow CdaR to sense changes in osmolarity and convey the signal input to CdaA. GImM in contrast could serve to connect cell wall biosynthesis to osmoregulation that need to be intertwined to allow adaption of the cell to turgor changes. We therefore aimed to deepen the knowledge of the role of CdaR and GImM in regulation of CdaA activity.

Furthermore, the identifications of novel c-di-AMP regulated processes in L. monocytogenes is crucial to elucidate the signaling network. Therefore, the identification of c-di-AMP binding proteins in L. monocytogenes, the investigation of their function and regulation is another focus of this study. The main interest in this context were proteins, like potassium transporter, that play important roles in osmoregulation.

c-di-AMP has been shown to impact a variety of cellular functions, ranging from osmoregulation, cell wall biosynthesis to DNA damage repair. To broaden the understanding of the c-di-AMP signaling network, we analyzed the effects of a cdaA deletion mutant in L. monocytogenes. The aim was to find novel pathways that are transcriptional or translational regulated by c-di-AMP using a transcriptomic and proteomic approach.

Finally, c-di-AMP is synthesized by other types of DACs. The second most abundant class is of the DNA-binding DisA type. Hence, we wanted to investigate the regulation of DisA from $B$. subtilis in the context of osmoregulation. We hypothesized that DNA-binding of DisA might be regulated by macromolecular crowding upon osmotic stress, as it has been shown for other DNA-binding proteins that are involved in osmoregulation.

Summarizing, the main goal of the following work was to expand the current knowledge about the fascinating second messenger c-di-AMP and its important regulatory functions in L. monocytogenes. 


\title{
2. The YbbR domain-containing protein CdaR regulates di- adenylate cyclase activity of CdaA
}

Johannes Gibhardt, Ole Hinrichs, Volkhard Kaever, and Fabian M. Commichau

Author contribution:

$J G$ and $\mathrm{OH}$ performed the experiments. VK performed the c-di-AMP measurements. JG and FMC wrote the manuscript.

\begin{abstract}
The regulation of osmotic homeostasis in Firmicutes is regulated by the second messenger c-di-AMP. This signal molecule has been shown to regulate various osmolyte transport systems for the uptake of potassium or glycine betaine in important bacteria, like Bacillus subtilis, Listeria monocytogenes or Staphylococcus aureus. One of the most important open questions is still the regulation of synthesis and degradation of c-di-AMP, by diadenylate cyclases (DACs) and phosphodiesterases (PDEs), respectively. Here, we show that $c d a R$, which is located in an operon with $c d a A$, is involved in osmotic regulation of L. monocytogenes. A $\triangle c d a R$ mutant was identified in a phenotype microarray to have an altered metabolic activity under osmotic stress conditions and shows a decreased growth rate if grown with the osmolytes $\mathrm{NaCl}, \mathrm{KCl}$, or sorbitol. Furthermore, we show that the YbbR domains of CdaR are involved in regulation of c-di-AMP levels and osmotic adaptation. Topology determinations show that these domains are located towards the outside, potentially sensing mechanic forces in the bacterial cell wall due to turgor changes. In addition, we demonstrate in an E. coli-based screening system the ability of CdaR and of the phosphoglucosamine mutase GImM to influence DAC activity of CdaA. Additionally, we show that both, membrane localization and the YbbR domains of CdaR are important for proper function of the regulatory process. Moreover, suppressor mutants that show increased resistance to osmotic stress impact the stress sigma Factor SigB, leading to its inactivation. Finally, we show a potential interaction network between synthesis and degradation systems of the $L$. monocytogenes c-di-AMP metabolic network.
\end{abstract}




\section{Introduction}

The second messenger c-di-AMP has been discovered a decade ago in the crystal structure of the DNA-integrity scanning protein DisA (Witte et al., 2008). Interestingly, it is the first second messenger that is both, essential and toxic at the same time (Corrigan et al., 2011; Gundlach et al., 2015b; Mehne et al., 2013; Woodward et al., 2010). c-di-AMP is also a special second messenger, since it has been shown to regulate a biological process on more than one level - by regulating the expression of a gene and the activity of the protein. It binds to the kimA riboswitch, preventing gene expression of the $k \operatorname{tr} A B$ genes that encode for the high affinity potassium transporter KtrAB and it also binds KtrA directly, inhibiting the potassium import (Corrigan et al., 2013; Gundlach et al., 2017; Kim et al., 2015; Nelson et al., 2013). This two-level regulation, demonstrates that regulation of potassium import needs to be tightly controlled, because it is one of the most important tools bacteria have to adapt to changes in extracellular osmolarity as a first response (Sleator \& Hill, 2002). Adaptation to changes in osmolarity is a complex process involving not only regulated uptake of potassium but furthermore the later replacement of potassium with more compatible solutes, such as glycine betaine and also the adaption of cell wall and membrane to allow the bacterial cell envelope to adapt accordingly to the changes in turgor (Misra et al., 2013; Rojas et al., 2014; Sévin \& Sauer, 2014; Wood et al., 2001). Uptake or synthesis of compatible solutes is a widespread tool and bacteria, as Escherichia coli, B. subtilis, L. monocytogenes, S. aureus, Salmonella typhimurium or Streptococcus pneumoniae all developed mechanisms for synthesis or uptake of different compatible solutes (Sleator \& Hill, 2002). Interestingly, c-di-AMP does not only regulate potassium import systems, but also the import of compatible solutes like glycine betaine or carnitine (Huynh et al., 2016; Schuster et al., 2016; Zeden et al., 2018). c-di-AMP can therefore be described as the major regulator of osmotic homeostasis in Firmicutes. Despite the increasing knowledge about its function and target proteins, a lot is still unknown. Recently, conditions were found, under which c-di-AMP is dispensable (Gundlach et al., 2017; Whiteley et al., 2015; Whiteley et al., 2017; Zeden et al., 2018). Intriguingly, those conditions are defined growth conditions, under which osmolyte availability is limited. Gundlach et al. could show that c-di-AMP levels in B. subtilis concord with the external concentrations of potassium and also the nitrogen source (Gundlach et al., 2015b; Gundlach et al., 2017). The bacteria must therefore have systems in place that can detect the osmotic state they are living in and adjust synthesis and degradation of c-di-AMP accordingly. This is also one of the major open questions: how do bacteria sense changes in osmolarity and how do they confer these stimuli to altered activity or expression of the DACs and PDEs. For the DisA-type cyclase, it has been shown that binding of DNA, especially complex holiday junction structure inhibits its enzymatic function (Bejerano-Sagie et al., 2006; Witte et al., 2008). It is unclear though, how this might be connected to changes in osmolarity. B. subtilis is of special interest for c-di-AMP research, since it is the only bacterium that has three DACs, DisA, CdaA and the sporulation specific CdaS (Mehne et al., 2014). CdaS is only highly expressed after sporulation initiation (Nicolas et al., 2012). It features a C-terminal DAC domain and two N-terminal helices. Truncation of those helices leads to hyperactivity of the catalytic domain and they are therefore designated as autoinhibitory domains (Mehne et al., 2014). The regulatory effect of these $\mathrm{N}$-terminal helices in vivo is unknown and remains to be elucidated. The CdaA type of DACs, which is also referred to DacA, is a membrane bound enzyme (Corrigan et al., 2011; Mehne et al., 2013; Rosenberg et al., 2015; Woodward et al., 2010). It consists of three N-terminal transmembrane (TM) domains, a coiled-coiled domain, followed by the DAC domain and another C-terminal coiled-coiled domain. $c d a A$ is genetically conserved with $c d a R$, the gene encoding the CdaR protein and $g / m M$ encoding the phosphoglucosamine mutase GImM. CdaR has an N-terminal domain, designated as signal peptide and four YbbR domains of unknown function. GImM on the other 
side is a cytosolic protein that catalyzes the conversion of glucosamine-6-phosphate to glucosamine-1-phosphate and is therefore one of the early enzymes for peptidoglycan synthesis and essential (Mehne et al., 2013; Rismondo et al., 2016; Tosi et al., 2019). Interestingly, it has been shown that GImM affects CdaA activity in Lactococcus lactis (Zhu et al., 2016), demonstrating a regulatory cross-talk between c-di-AMP metabolism (and therefore osmotic regulation) and cell wall biosynthesis. In the present study, we show that both the CdaR and GImM proteins are able to modulate CdaA activity in vivo. Furthermore, we show that the YbbR domains of CdaR are important for the regulation of CdaA activity. They are located outside of the cell and might act as a sensor for turgor changes in the cell envelope. We furthermore show interactions between multiple parts of the c-di-AMP metabolic network, demonstrating potential cross-talk between the synthesis and degradation machineries.

\section{Experimental procedures}

Bacterial strains and growth conditions - L. monocytogenes EGD-e and its derivatives were cultivated in $\mathrm{BHI}$ medium (Sigma-Aldrich) at $37^{\circ} \mathrm{C}$ and $220 \mathrm{rpm}$ if not specified otherwise. E. coli was grown in LB medium at $37^{\circ} \mathrm{C}$ and $220 \mathrm{rpm}$ (strains see Tab. 2.1). For agar plates, medium was supplemented with $15 \mathrm{~g} / \mathrm{l}$ Bacto Agar (Difco). Antibiotics and medium supplements were used with the following concentrations, if indicated: erythromycin $(5 \mu \mathrm{g} / \mathrm{ml})$, kanamycin $(50 \mu \mathrm{g} / \mathrm{ml})$, ampicillin $(100 \mu \mathrm{g} / \mathrm{ml})$, chloramphenicol $(30 \mu \mathrm{g} / \mathrm{ml})$, streptomycin $(100 \mu \mathrm{g} / \mathrm{ml}), \mathrm{X}-\mathrm{Gal}(100 \mu \mathrm{g} / \mathrm{ml}$; 5-bromo-4-chloro-3-indolyl- $\beta$-D-galactopyranoside; Sigma-Aldrich), IPTG (1 mM or $50 \mu \mathrm{M}$; Isopropyl $\beta$-D-1-thiogalactopyranoside; Sigma-Aldrich), L-arabinose $(0.005 \%(w / v))$. For E. coli experiments under defined conditions, a modified, potassium defined M9 medium was used, as described elsewhere (see chapter 3). As a minimal medium for L. monocytogenes the LSM medium (Whiteley et al., 2017) was used with the following minor changes (equimolar substitutions): riboflavin-5'-monophosphate instead of Riboflavin, L-isoleucine, L-methionine and L-valine instead of the DL-enantiomers and L-cysteine $\cdot \mathrm{HCL} \cdot \mathrm{H}_{2} \mathrm{O}$ instead of L-cysteine $\cdot 2 \mathrm{HCL}$. For pouring minimal medium agar plates, $2 \mathrm{X}$ concentrated medium was pre-warmed to $37^{\circ} \mathrm{C}$ and mixed with to $70^{\circ} \mathrm{C}$ pre-warmed $2 \times$ Bacto agar, directly before pouring the plates.

DNA manipulation - DNA amplification via PCR and transformation of $E$. coli was performed using standard procedures (Sambrook et al., 1989). DNA fragments were purified using the PCR purification kit (Qiagen) and plasmid DNA was extracted using the NucleoSpin Plasmid Kit (Macherey and Nagel). Commercially available restriction enzymes, T4 DNA ligase and DNA polymerases were used as recommended by the manufacturers. DNA sequences were determined by the dideoxy chain termination method (Microsynth, Göttingen, Germany). Chromosomal DNA of L. monocytogenes was isolated using the NucleoSpin Microbial DNA Kit (Macherey and Nagel). Oligonucleotides were purchased from Sigma-Aldrich (Germany).

Tab. 2.1 Strains

\begin{tabular}{|c|c|c|c|}
\hline Name & Genotype & Description/Construction & Reference \\
\hline \multicolumn{4}{|l|}{ E. coli } \\
\hline BL21(DE3) & $\begin{array}{l}\mathrm{F}^{-} \text {ompT gal dcm lon } h s d S_{B}\left(r_{B}^{-} m_{B}^{-}\right) \lambda(\mathrm{DE} 3[\text { lacl } \\
\text { lacUV5-T7p07 ind1 sam7 nin5]) }\left[\text { malB }^{+}\right]_{\mathrm{K}-12}\left(\lambda^{\mathrm{S}}\right) \\
\mathrm{F}^{-} \text {cya99 araD139 galE15 galK16 rpsL1 }\left(\mathrm{Str}^{\mathrm{R}}\right) h s d R 2 \\
\text { mcrA1 mcrB1 }\end{array}$ & $\begin{array}{l}\text { Protein expression } \\
\text { BACTH assays }\end{array}$ & $\begin{array}{l}\text { Stratagene } \\
\text { Karimova et al., } \\
1998\end{array}$ \\
\hline
\end{tabular}




\begin{tabular}{|c|c|c|c|}
\hline Name & Genotype & Description/Construction & Reference \\
\hline XL1-Blue & 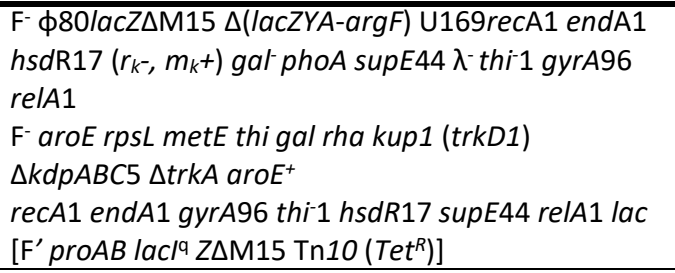 & $\begin{array}{l}\text { PhoA and LacZ assays } \\
\text { Potassium importer defi- } \\
\text { cient strain } \\
\text { Cloning }\end{array}$ & $\begin{array}{l}\text { Sambrook et al., } \\
1989 \\
\text { Stumpe \& Bakker, } \\
1997 \\
\text { Stratagene }\end{array}$ \\
\hline \multicolumn{4}{|c|}{ L. monocytogenes } \\
\hline EGD-e & Wild type & Serotype $1 / 2$ a strain & $\begin{array}{l}\text { Laboratory collec- } \\
\text { tion }\end{array}$ \\
\hline LMJR45 & $\Delta c d a R$ & $\begin{array}{l}\text { Chromosomal deletion of } \\
\text { cdaR }\end{array}$ & $\begin{array}{l}\text { Rismondo et al., } \\
2016\end{array}$ \\
\hline BPL16 & $\Delta c d a R$ attB::P Pelp - lacO-cdaR lacl neo & $\begin{array}{l}\text { IPTG-dependent } c d a R \text { ex- } \\
\text { pression }\end{array}$ & $\begin{array}{l}\text { Rismondo et al., } \\
2016\end{array}$ \\
\hline BPL45 & attB::Phelp-lacO-MCS lacl neo & pIMK3 $\rightarrow$ EGD-e & This work \\
\hline BPL46 & $\Delta c d a R$ attB:: $\mathrm{P}_{\text {help }}-$ lacO-MCS lacl neo & pIMK3 $\rightarrow$ LMJR45 & This work \\
\hline BPL47 & $\begin{array}{l}\Delta c d a R \text { attB::Phelp-lacO-cdaR (aa 32-452; } \Delta \mathrm{TM} \text { ) lacl } \\
\text { neo }\end{array}$ & pBP255 $\rightarrow$ LMJR45 & This work \\
\hline BPL48 & $\begin{array}{l}\Delta c d a R \text { attB::Phelp-lacO-cdaR (aa 1-320; } \triangle \mathrm{YbbR} \text { do- } \\
\text { main 4) lacl neo }\end{array}$ & pBP256 $\rightarrow$ LMJR45 & This work \\
\hline BPL49 & $\begin{array}{l}\Delta c d a R \text { attB::Phelp-lacO-cdaR (aa 1-230; } \Delta \mathrm{YbbR} \text { do- } \\
\text { main 3-4) lacl neo }\end{array}$ & pBP257 $\rightarrow$ LMJR45 & This work \\
\hline BPL50 & $\begin{array}{l}\Delta c d a R \text { attB::Phelp-lacO-cdaR (aa 1-130; } \triangle \mathrm{YbbR} \text { do- } \\
\text { main 2-4) lacl neo }\end{array}$ & pBP258 $\rightarrow$ LMJR45 & This work \\
\hline BPL51 & $\begin{array}{l}\Delta c d a R \text { attB::Phelp-lacO-cdaR (aa 1-33; } \triangle \mathrm{YbbR} \text { do- } \\
\text { main 1-4) lacl neo }\end{array}$ & pBP259 $\rightarrow$ LMJR45 & This work \\
\hline BPL52 & $r s b \cup 128 \mathrm{G}>\mathrm{T}(\mathrm{E} 116 \mathrm{X})$ & $\begin{array}{l}\text { EGD-e suppressor mu- } \\
\text { tant, isolated on LSM + } \\
0.5 \text { M D-sorbitol } \\
\text { EGD-e suppressor mu- }\end{array}$ & This work \\
\hline BPL53 & sigB 178GT>CC 171_175delCAAGT (I71X) & $\begin{array}{l}\text { tant, isolated on LSM + } \\
0.5 \text { M D-sorbitol }\end{array}$ & This work \\
\hline BPL55 & $\Delta c d a R$ pIMK3 rsbU 665C $>$ T (A222V) pdeA 1698C $>\mathrm{T}$ & $\begin{array}{l}\text { BPL46 suppressor mu- } \\
\text { tant, isolated on LSM + } \\
0.25 \mathrm{M} \mathrm{NaCl}\end{array}$ & This work \\
\hline BPL56 & $\Delta c d a R$ pIMK3 Imo1515 261delC (L115X) & $\begin{array}{l}\text { BPL46 suppressor mu- } \\
\text { tant, isolated on LSM + } \\
0.25 \mathrm{M} \mathrm{NaCl}\end{array}$ & This work \\
\hline BPL57 & $\Delta c d a R$ pIMK3 rsbU 532delG (L189X) & $\begin{array}{l}\text { BPL46 suppressor mu- } \\
\text { tant, isolated on LSM + } \\
0.25 \mathrm{M} \mathrm{NaCl}\end{array}$ & This work \\
\hline BPL58 & $\begin{array}{l}\Delta c d a R \text { pIMK3 Imo0892/Imo0893 intergenic } \\
\left(929,827 A>G ; 929,831 \_929,832 \text { insAA; } 929,832 T>A ;\right. \\
\text { 929,836A>G; 929,842T>A; 929,845GT>TA; } \\
\text { 929,854AA>GT; 929,857T>A; 929,861C>T; } \\
\text { 929,866T>A; 929,868C>A; 929,870TA>AG; } \\
\text { 929,879A>G; 929,881GG>TT) Imo1432/Imo1433 in- } \\
\text { tergenic }(1,464,183 C>T)\end{array}$ & $\begin{array}{l}\text { BPL46 suppressor mu- } \\
\text { tant, isolated on LSM + } \\
0.25 \mathrm{M} \mathrm{KCl}\end{array}$ & This work \\
\hline BPL59 & $\begin{array}{l}\text { AcdaR pIMK3 Imo1515 412_422dupTTTTATATTTA; } \\
\text { 423delA (X141fs173) }\end{array}$ & $\begin{array}{l}\text { BPL46 suppressor mu- } \\
\text { tant, isolated on } \mathrm{LSM}+ \\
0.25 \mathrm{M} \mathrm{KCl}\end{array}$ & This work \\
\hline BPL77 & $\Delta c d a A$ & pBP352 $\rightarrow \rightarrow$ EGD-e & This work \\
\hline
\end{tabular}

Plasmid construction - For the determination of the membrane topology of the CdaR protein, plasmids pBP250 (cdaR), pBP251 (cdaR aa 1-33; $\triangle$ YbbR domains), pBP252 (cdaR aa 34-452; $\triangle \mathrm{TM}$ domain), pBP253 (prkA; Imo1820) and pBP254 (prfA; Imo0200) were constructed on the base of the pKTop plasmid (Karimova et al., 2009; plasmids are listed in Tab. S2.1). This plasmid allows the IPTG-dependent expression of fusion proteins with a C-terminal phoA gene and the lacZ $\alpha$ - 
fragment. The genes were amplified using oligonucleotide pairs JH121/122, JH121/124, $\mathrm{JH} 123 / \mathrm{JH} 122, \mathrm{JH} 126 / 127$ and JH128/JH129, respectively (oligonucleotides are listed in Tab. S2.2). The resulting DNA fragments were digested with $\mathrm{BamHI} / \mathrm{Kpnl}$ and ligated to $\mathrm{pKTop}$, digested with the same enzymes. To analyze the effect of the membrane localization and the role of the YbbR domains in vivo, plasmids pBP255 (cdaR aa 32-452; $\triangle \mathrm{TM}$ ), pBP256 (cdaR; aa 1-320; $\triangle \mathrm{YbbR} 4$ ), pBP257 (cdaR; aa 1-230; $\triangle$ YbbR 3-4), pBP258 (cdaR; aa 1-130; $\triangle$ YbbR 2-4), pBP259 (cdaR; aa 1-33; $\triangle$ YbbR 1-4) were constructed on the base of the pIMK3 plasmid (Monk et al., 2008). The pIMK3 plasmid allows for IPTG-dependent, ectopic expression of genes in L. monocytogenes EGD-e by integration of the whole plasmid in the tRNA ${ }^{\text {Arg }}$ locus (Imot17). The genes were amplified using the oligonucleotide pairs $\mathrm{JH} 130 / \mathrm{JH} 22, \mathrm{JH} 21 / \mathrm{JH} 132, \mathrm{JH} 21 / \mathrm{JH} 133, \mathrm{JH} 21 / \mathrm{JH} 134$ and JH21/JH131, respectively. The resulting DNA fragments were digested using $\mathrm{Ncol} / \mathrm{Sall}$ and ligated to $\mathrm{pIMK3}$, digested with the same enzymes. For the analysis of CdaA activity in $E$. coli and the impact of GlmM, CdaR and CdaR variants on its activity, plasmids pBP387 (cdaA-cdaR), pBP388 (cdaA-cdaR-glmM), pBP389 (cdaA-gImM), pBP260 (cdaA-cdaR aa 34-452; $\triangle \mathrm{TM}$ ), pBP261 (cdaA-cdaR aa 1-320; $\triangle \mathrm{YbbR}$ 4), pBP262 (cdaA-cdaR aa 1-230; $\triangle$ YbbR 3-4), pBP263 (cdaA-cdaR aa 1-130; $\triangle$ YbbR 2-4) and pBP264 (cdaA-cdaR aa 1-33; $\triangle T$ T) were constructed based on the pBAD33 plasmid (Guzman et al., 1995). The genes for pBP387, pBP261, pBP262, pBP263 and pBP264 were amplified using oligonucleotide pairs $\mathrm{JH} 51 / \mathrm{JH} 103, \mathrm{JH} 51 / \mathrm{JH} 138, \mathrm{JH} 51 / \mathrm{JH} 139, \mathrm{JH} 51 / \mathrm{JH} 140$ and $\mathrm{JH} 51 / \mathrm{JH} 137$, respectively. The resulting DNA fragments were digested with $\mathrm{Xbal} / \mathrm{Pstl}$ and ligated to pBAD33, digested with the same enzymes. For pBP388 and pBP389, the gImM gene was amplified with the oligonucleotides $\mathrm{JH} 104$ and $\mathrm{JH} 105$ and the resulting DNA fragments digested with Pstl/HindIII. They were ligated to pBP387 (pBAD33-cdaAR) or pBP370 (pBAD33-cdaA) digested with the same enzymes, respectively (Quintana et al., 2019). For the construction of plasmid pBP260, the $c d a A$ gene and the cdaR gene - lacking the first 33 amino acids - were amplified using oligonucleotide pairs $\mathrm{JH} 51 / \mathrm{JH} 135$ and $\mathrm{JH} 136 / \mathrm{JH} 103$, respectively. The two products were fused via splicing by overlap extension PCR (Horton et al., 1990) using oligonucleotides JH51 and JH103. The resulting DNA fragment was digested with $X b a l / P s t l$ and ligated to pBAD33, digested with the same enzymes. To analyze the protein-protein interaction of different proteins of the c-di-AMP metabolism, plasmids pBP359 to pBP362 ( $g / m M$; Imo2118), pBP269 to pBP272 (pdeA; Imo0052), pBP273 to pBP276 (pgpH; Im1466) and pBP277 to pBP280 (kimA; Imo2130) were constructed on basis of the BACTH plasmids pUT18, pUT18C, p25-N and pKT25, respectively (Claessen et al., 2008; Karimova et al., 1998). These plasmids allow the construction of either C- or N-terminal T18 or T25 domains of the cAMP adenylate cyclase to investigate protein-protein interactions in $E$. coli strains with cya mutations, lacking the native CAMP AC gene, like the BTH101 strain (Karimova et al., 1998). The genes for glmM, pdeA, pgpH and kimA were amplified using oligonucleotide pairs FC336/FC337, $\mathrm{JH} 160 / \mathrm{JH} 161, \mathrm{JH} 162 / \mathrm{JH} 163$ and $\mathrm{JH} 164 / \mathrm{JH} 165$, respectively. Resulting DNA fragments were digested with $\mathrm{Xbal} / \mathrm{Kpnl}$ and ligates to pUT18, pUT18C, p25-N or pKT25, which were digested with the same enzymes, respectively. For the chromosomal deletion of the $c d a A$ gene, pBP352 was constructed. The up- and downstream regions of $c d a A$, while sparring the $c d a A$ ORF out, were amplified using oligonucleotide pairs $\mathrm{JH05} / \mathrm{JHO6}$ and $\mathrm{JH07} / \mathrm{JH} 08$, respectively. The resulting $\mathrm{PCR}$ products were fused by SOE PCR using oligonucleotides $\mathrm{JHO5}$ and $\mathrm{JH} 08$, digested with EcoRI and BamHI and ligated to PMAD (Arnaud et al., 2004; Horton et al., 1990), which was digested using the same enzymes. Plasmids pBP223 and pBP131 were constructed for overexpression of N-terminal-Strep-tagged $C d a R(\triangle a a b-28$ ) and PrfA, respectively. The $c d a R$ and $p r f A$ genes were amplified using oligonucleotide pairs JR56/JR28 and FC206/FC205, respectively. The resulting DNA fragments were digested using $S a c l / B a m H I$ and $S a c l / B g / l l$, respectively and ligated to pGP172, digested with Sacl/BamHI (Merzbacher et al., 2004). 
L. monocytogenes strain construction - For the investigation of the impact of CdaR on c-di-AMP synthesis and adaptation to osmotic stress in vivo, L. monocytogenes mutants were constructed as follows. Electrocompetent cells were prepared as described elsewhere (Monk et al., 2008). Shortly, the EGD-e wt or the $\Delta c d a R$ mutant (LMJR45) were cultured in BHI until early exponential phase, the cell wall synthesis was at this point inhibited by $10 \mu \mathrm{g} / \mathrm{ml}$ ampicillin for two hours at $37^{\circ} \mathrm{C}$ and $220 \mathrm{rpm}$. Afterwards, the cells were washed in decreasing amounts of SGW buffer $(10 \%$ $(w / v)$ glycerol, $500 \mathrm{mM}$ sucrose, $\mathrm{pH} 7)$ and incubated with $10 \mu \mathrm{g} / \mathrm{ml}$ of lysozyme for 30 minutes at $37^{\circ} \mathrm{C}$. The cells were washed one more time and frozen as aliquots in liquid nitrogen. For the electroporation of plasmid DNA, $50 \mu \mathrm{l}$ of electrocompetent cells were added on 1-5 $\mu \mathrm{g}$ precipitated DNA, incubated for $10 \mathrm{~min}$ on ice and transferred to a chilled $0.1 \mathrm{~cm}$ gap electroporation cuvette (BioRad). Electroporation was performed at $18 \mathrm{kV} / \mathrm{cm}$ using the MicroPulser electroporator (BioRad). Immediately after the electroporation, $1 \mathrm{ml}$ of to $37^{\circ} \mathrm{C}$ pre-warmed medium was added and the cells incubated for $1.5 \mathrm{~h}$ at $30^{\circ} \mathrm{C}$ without agitation. Eventually, bacteria were plated on selective media and correct chromosomal integration of the PIMK3 derivatives was confirmed via PCR and integrity of the MCS by Sanger sequencing. With this protocol, the following strains were constructed: BPL45 (EGD-e + pIMK3 empty plasmid), BPL46 ( $\Delta c$ cdaR + pIMK3 empty plasmid), BPL47 $(\Delta c d a R+$ pBP255 (pIMK3-cdaR $\Delta \mathrm{TM}))$, BPL48 ( $\triangle c d a R+$ pBP256 (pIMK3-cdaR $\Delta$ YbbR 4)), BPL49 ( $\Delta c d a R+$ pBP257 (pIMK3-cdaR $\triangle \mathrm{YbbR} 3-4))$, BPL50 ( $\Delta c$ daR + pBP258 (pIMK3-cdaR $\Delta$ YbbR 2-4)), BPL51 ( $\triangle c d a R+$ pBP259 (pIMK3-cdaR $\triangle$ YbbR 1-4)). For the chromosomal deletion of the $c d a A$ gene, strain BPL77 was constructed as follows. The EGD-e wt was electroporated with plasmid pBP352 (pMAD- $\triangle c d a A$ ) and plated on LSM medium with erythromycin and X-Gal at $30^{\circ} \mathrm{C}$ for up to $72 \mathrm{~h}$. Single, blue colonies were streaked on the same medium and incubated for up to $72 \mathrm{~h}$ at $42^{\circ} \mathrm{C}$ to force integration into the $c d a A$ locus. Several blue colonies were used to inoculate $5 \mathrm{ml}$ of LSM without antibiotics at $30^{\circ} \mathrm{C}$ for $4 \mathrm{~h}$, temperature was shifted to $42^{\circ} \mathrm{C}$ for $6 \mathrm{~h}$, after which serial dilutions were plated on LSM medium with X-Gal and incubated at $37^{\circ} \mathrm{C}$ for up to $72 \mathrm{~h}$. Erythromycin-sensitive, $\mathrm{X}$-Gal negative bacteria that did grow on LSM medium but not on $\mathrm{BHI}$ were subjected to colony PCR as described elsewhere (Dussurget et al., 2002). cdaA deletion and absence of ectopic suppressor mutations was confirmed by whole genome sequencing (WGS) and Sanger sequencing and the strain designated BPL77.

Phenotypic microarray screening - To screen for a phenotype of the $\Delta c$ daR mutant (LMJR45), a Phenotype MicroArray (PM; Biolog Inc.; Bochner et al., 2001) was employed. The L. monocytogenes wt or the $\triangle c d a R$ mutant were streaked to single colonies on $\mathrm{BHI}$ agar plates $\left(37^{\circ} \mathrm{C}\right.$ overnight). $10 \mathrm{ml}$ of $\mathrm{BHI}$ were incubated from a single colony at $37^{\circ} \mathrm{C}$ and $220 \mathrm{rpm}$ to an $\mathrm{OD}_{600}$ of $0.4-$ $0.5 .9 \mathrm{ml}$ of those cultures were harvested by centrifugation at $3300 \mathrm{~g}$ for $10 \mathrm{~min}$ at $4^{\circ} \mathrm{C}$ and the pellet resuspended in $1 \mathrm{ml} \mathrm{BHI}$ with $25 \%(\mathrm{w} / \mathrm{v})$ glycerol, frozen in liquid nitrogen and stored at $80^{\circ} \mathrm{C}$. A $10 \mu$ inoculation loop was used to freshly re-streak the bacteria from cryo cultures on $\mathrm{BHI}$ agar plates to a bacterial lawn, prior to each PM and incubated for $20 \mathrm{~h}$ at $37^{\circ} \mathrm{C}$. The cells were scratched evenly from the bacterial lawn, resuspended in the manufactures inoculation fluid and adjusted to an $\mathrm{OD}_{600}$ of 0.3 in $1 \mathrm{ml}$ of the inoculation fluid. The remaining treatment was performed according to the recommendations of the manufacturer. The cells were incubated on the different PM 96-well plates (Microtest Plate 96-Well,F, Sarstedt) with $100 \mu \mathrm{l}$ of cells per well for 48 hours at $37^{\circ} \mathrm{C}$ with orbital shaking $(237 \mathrm{cpm}, 4 \mathrm{~mm})$ and the $O D_{590}$ was measured in $30 \mathrm{~min}$ intervals using an Epoch2 multiwell reader, equipped with the Gen5 software (02.09.2001; BioTek Instruments).

Osmotic stress and bacterial growth - To evaluate the osmotic phenotype of the $\Delta c d a R$ mutant (LMJR45), seen in the Biolog PM, the growth of the mutant was compared to the EGD-e wt under osmotic stress. Bacteria were pre-cultured overnight from single colonies in $5 \mathrm{ml} \mathrm{BHI}$ medium at 
$37^{\circ} \mathrm{C}$ and $220 \mathrm{rpm} .10 \mathrm{ml} \mathrm{BHI}$ were inoculated from the pre-cultures to an $\mathrm{OD}_{600}$ of 0.1 and grown at $37^{\circ} \mathrm{C}$ and $220 \mathrm{rpm}$ until they reached an $\mathrm{OD}_{600}$ of $0.4-0.8$. The optical density was adjusted to 0.2 and $100 \mu \mathrm{l}$ of the cell suspension was pipetted into 96-well plates, containing $100 \mu \mathrm{l} \mathrm{BHI}$ medium with or without various concentrations of $\mathrm{NaCl}, \mathrm{KCl}$, or D-sorbitol. The final concentrations were $0,0.25,0.5,0.75,1,1.5$ and $2 \mathrm{M}$ of the different osmolytes. Bacteria were grown at $37^{\circ} \mathrm{C}$ and $237 \mathrm{cpm}(4 \mathrm{~mm})$ agitation using an Epoch 2 multiwell platereader, equipped with the Gen5 software (02.09.2001; BioTek Instruments) and the optical density was determined in 15 min intervals at $600 \mathrm{~nm}$. Growth rates of the exponential phases were determined as described elsewhere (see chapter 3 ) and plotted against the osmolyte concentration.

Protein purification and generation of an anti-CdaR and anti-PrfA antiserum - Plasmids PBP223 and pBP131 were used to overexpress the N-terminally Strep-tagged CdaR ( $\triangle$ aa $1-28)$ and PrfA from L. monocytogenes, respectively, using BL21(DE3) as described previously for CdaA (Rosenberg et al., 2015). The proteins were purified with the Strep-tag II-Strep-Tactin purification system (IBA, Göttingen, Germany) and used for the generation of polyclonal antibodies in rabbits (Seqlab, Göttingen, Germany). The antibodies were diluted 1:1000 for Western blot analyses.

Western blot analysis of CdaR localization - Strains EGD-e (wt), BPL77 ( $\triangle c d a A)$ and LMJR45 ( $\Delta c d a R)$ were cultivated in $100 \mathrm{ml} \mathrm{LSM}$ at $37^{\circ} \mathrm{C}$ and $220 \mathrm{rpm}$ from an $\mathrm{OD}_{600}$ of 0.05 to about 0.5 and harvested by centrifugation for $10 \mathrm{~min}$ at $3300 \mathrm{~g}$ and $4^{\circ} \mathrm{C}$. Pellets were washed once in $10 \mathrm{ml}$ ZAP (50 mM Tris- $\mathrm{HCl}, \mathrm{pH} 7.5,200 \mathrm{mM} \mathrm{NaCl}$ ). Pellets were resuspended in $400 \mu \mathrm{l}$ ZAP with DNase I (0.5 $\mathrm{U} / \mathrm{ml}$; Sigma-Aldrich) and cOmplete EDTA-free Protease Inhibitor Cocktail (1 tablet/50 ml; SigmaAldrich). $200 \mu \mathrm{l}$ of cell suspension were added to $0.5 \mathrm{~g}$ glass beads ( $0.1 \mathrm{~mm}$ diameter; Carl Roth) and disrupted using a Tissuelyserll (Qiagen) for $15 \mathrm{~min}$ at $30 \mathrm{~Hz}$ at $4^{\circ} \mathrm{C} .600 \mu \mathrm{l}$ of the buffer was added to the tubes and incubated for $10 \mathrm{~min}$ on ice. Samples were centrifuged for $2 \mathrm{~min}$ at $20000 \mathrm{~g}$ and $4^{\circ} \mathrm{C}$ and the supernatant transferred to a new tube (whole cell lysate). $1 \mathrm{ml}$ was subjected to ultracentrifugation at $235000 \mathrm{~g}$ for $1 \mathrm{~h}$ at $4^{\circ} \mathrm{C}$. The supernatant was transferred to a new tube (cytosolic fraction) and the pellet resuspended in $1 \mathrm{ml}$ of buffer. After a second ultracentrifugation for $30 \mathrm{~min}$, the supernatant was transferred to a new tube and the pellet resuspended (membrane fraction) in $100 \mu \mathrm{l}$ of the buffer with $17 \mathrm{mM}$ CHAPS (3-[(3-Cholamidopropyl)dimethylammonio]-1propanesulfonate hydrate; Sigma-Aldrich). Protein concentrations were determined by Bradford assay (Sambrook et al., 1989) and $10 \mu \mathrm{g}$ of protein extracts separated on 12\% SDS polyacrylamide gels. Proteins were transferred on a polyvinylidene difluoride membrane (Bio-Rad) by electroblotting. Proteins were detected by specific antibodies against CdaA (Rosenberg et al., 2015), CdaR or PrfA, respectively. The primary antibodies were visualized by using anti-rabbit IgG (immunoglobulin G) AP (alkaline phosphatase) secondary antibodies (Promega) and the CDP* detection system (Roche Diagnostics).

Determination of CdaR membrane topology - To determine the location of the YbbR domains of the CdaR regulatory protein, plasmids pBP250, pBP251, pBP252, pBP253, pBP254 and the pKTop empty plasmid. E. coli $\mathrm{DH} 5 \alpha$ was subsequently transformed with the constructed plasmids. This strain has a deletion of the phoA gene, encoding the alkaline phosphatase PhoA and the $\triangle($ lacZ)M15 mutation that allows complementation of the $\beta$-galactosidase with the LacZ $\alpha$-fragment. The bacteria were grown in LB supplemented with kanamycin from an $\mathrm{OD}_{600}$ of 0.1 to $0.3-$ 0.6 from overnight cultures. The $\mathrm{OD}_{600}$ was adjusted to 0.1 and $5 \mu$ of the cell suspension was streaked out on LB plates containing kanamycin, $1 \mathrm{mM} \mathrm{IPTG}, 80 \mathrm{\mu g} / \mathrm{ml} \mathrm{X-phosphate} \mathrm{(5-Bromo-4-}$ chloro-3-indolyl phosphate disodium salt; Sigma-Aldrich), $100 \mu \mathrm{g} / \mathrm{ml}$ RedGal (6-Chloro-3-indolyl$\beta$-D-galactopyranoside; Sigma-Aldrich) and $50 \mathrm{mM}$ sodium phosphate buffer ( $\mathrm{pH} 7)$. The plates were incubated overnight at $37^{\circ} \mathrm{C}$ (Karimova et al., 2009). For the quantification of the alkaline 
phosphatase and $\beta$-galactosidase activity a slightly modified procedure as described by (Thongsomboon et $a l ., 2018$ ) was applied. DH5 $\alpha$ cells, containing the afore mentioned plasmids, were grown in LB medium containing kanamycin from overnight cultures from an $\mathrm{OD}_{600}$ of 0.1 to 0.5 0.6 at $37^{\circ} \mathrm{C}$ and $220 \mathrm{rpm}$. Four times $1.5 \mathrm{ml}$ cell culture were harvested by centrifugation at $20000 \mathrm{~g}, 4^{\circ} \mathrm{C}$ for $2 \mathrm{~min}$, two of them were washed in $1 \mathrm{ml} \mathrm{Z}$ buffer $\left(60 \mathrm{mM} \mathrm{Na} 2 \mathrm{HPO}_{4}, 40 \mathrm{mM}\right.$ $\mathrm{NaH}_{2} \mathrm{PO}_{4}, 10 \mathrm{mM} \mathrm{KCl}, 1 \mathrm{mM} \mathrm{MgSO}_{4}$ ) and the other two in $1 \mathrm{ml} 10 \mathrm{mM}$ Tris (pH 8). Cell pellets were resuspended in $1 \mathrm{ml}$ of the same buffers and lysed by addition of $50 \mu \mathrm{l} 0.1 \%(w / v)$ SDS, $50 \mu \mathrm{l}$ chloroform, vortexing for $10 \mathrm{~s}$ and incubation at room temperature for $10 \mathrm{~min}$. To determine the $\beta$ galactosidase activity, $200 \mu \mathrm{l}$ of a $4 \mathrm{mg} / \mathrm{ml}$ ONPG (o-Nitrophenyl- $\beta$-D-galactopyranosid; Sigma-Aldrich) in $\mathrm{Z}$ buffer solution was added. Reactions were stopped by addition of $500 \mu \mathrm{l} 1 \mathrm{M} \mathrm{Na}_{2} \mathrm{CO}_{3}$ when samples turned yellow, the time difference was noted and the $\mathrm{OD}_{415}$ of the supernatant was measured after $10 \mathrm{~min}$ of centrifugation at $20000 \mathrm{~g}$. Afterwards the specific $\beta$-galactosidase activity $[\mu \mathrm{mol} / \mathrm{min} / \mathrm{mg}]$ was calculated using the following formula: $\left(3.38 \cdot \mathrm{OD}_{415}\right) /\left(\mathrm{OD}_{600} \cdot \mathrm{V}(1.5) \cdot \Delta \mathrm{t}\right.$ [min]). For the determination of the alkaline phosphatase activity, $800 \mu$ l of lysed cells in $10 \mathrm{mM}$ Tris (pH8) were added to $100 \mu \mathrm{l}$ pNPP solution (SigmaFast solution $1 \mathrm{mg} / \mathrm{ml}$-Nitrophenyl phosphate in $200 \mathrm{mM}$ Tris $\mathrm{pH}$ 8; Sigma-Aldrich). The samples were incubated at $37^{\circ} \mathrm{C}$ until they turned yellow. Reactions were stopped by addition of $100 \mu \mathrm{l} 3 \mathrm{M} \mathrm{NaOH}$, the time was noted and the $\mathrm{OD}_{415}$ of the supernatant after 10 min of centrifugation (20000 g) was measured. Specific alkaline phosphatase activity $[\mu \mathrm{mol} / \mathrm{min} / \mathrm{mg}]$ was calculated using the following formula: $\left(6.46 \cdot \mathrm{OD}_{415}\right) /\left(\mathrm{OD}_{600}\right.$ $\cdot \mathrm{V}(1.5) \cdot \Delta \mathrm{t}[\mathrm{min}])$.

Analysis of the role of CdaR in osmotic adaptation - To assess the role of CdaR and the YbbR domains on adaptation of $L$. monocytogenes to osmotic stress, drop dilution experiments were performed. Strains BPL45, BPL46, BPL47, BPL48, BPL49, BPL50, BPL51 and BPL16 ( $\Delta c d a R+$ pIMK3cdaR) were streaked out on $\mathrm{BHI}$ agar plates containing kanamycin and incubated overnight at $37^{\circ} \mathrm{C}$. Single colonies were used to inoculate $5 \mathrm{ml}$ of LSM medium with kanamycin overnight at $37^{\circ} \mathrm{C}$ and $220 \mathrm{rpm}$. These cultures were used to inoculate $5 \mathrm{ml} \mathrm{LSM}$ medium with kanamycin to an $\mathrm{OD}_{600}$ of 0.1 . The bacteria were grown to an $\mathrm{OD}_{600}$ of $0.3-0.8$, adjusted to 0.1 and $10 \mu \mathrm{l}$ of serial dilutions were drop spotted on LSM agar plates with or without $0.25 \mathrm{M}$ of $\mathrm{NaCl}, \mathrm{KCl}$ or D-sorbitol. The plates were incubated at $37^{\circ} \mathrm{C}$ and photos taken every $24 \mathrm{~h}$.

Isolation of suppressor mutants and WGS - Emerging suppressor mutants were re-streaked twice on the same medium as isolated, cultured overnight and chromosomal DNA isolated as stated above. WG illumina sequencing was performed by the G2L Göttingen, the resulting reads analyzed using the Geneious software (Geneious Prime 2019.0.4 (https://www.geneious.com)) and potential mutations re-sequenced by Sanger Sequencing (Microsynth, Göttingen). The phenotypes of the mutants were confirmed by additional drop dilution assays.

Determination of intracellular c-di-AMP concentration - Strains BPL45, BPL46, BPL16, BPL47 and BPL51 were cultivated from single colonies overnight in 10 LSM with kanamycin. These pre-cultures were used to inoculate $75 \mathrm{ml}$ LSM medium with kanamycin and $1 \mathrm{mM} \mathrm{IPTG}$ to an $\mathrm{OD}_{600}$ of 0.1 . Bacteria were incubated at $37^{\circ} \mathrm{C}$ with agitation $(220 \mathrm{rpm})$ until they reached an $\mathrm{OD}_{600}$ of 0.5 0.6. At this time point $\left(\mathrm{t}_{0}\right)$ two times $10 \mathrm{ml}$ samples for the determination of the c-di-AMP concentration and two times $1 \mathrm{ml}$ samples for the determination of the protein concentration were taken (see below). Two times $27 \mathrm{ml}$ of each culture was transferred into new flasks with either $3 \mathrm{ml}$ of LSM or $3 \mathrm{ml}$ of LSM with $2.5 \mathrm{M} \mathrm{NaCl}$ (- and $+250 \mathrm{mM} \mathrm{NaCl}$, respectively). Cultures were incubated for another 25 minutes and once more samples were taken for c-di-AMP and protein amount determination. The $1 \mathrm{ml}$ samples for protein concentration determination were harvested by centrifugation at $20000 \mathrm{~g}$ for $1 \mathrm{~min}$ at $4^{\circ} \mathrm{C}$ and further processed as described earlier (Rismondo et al., 
2016). The $10 \mathrm{ml}$ samples for determination of the c-di-AMP concentration, were rapidly cooled by swirling in liquid nitrogen, centrifuged for $5 \mathrm{~min}$ at $3300 \mathrm{~g}$ and $4^{\circ} \mathrm{C}$ and the pellets frozen in liquid nitrogen. Samples were further processed, as described elsewhere (Rismondo et al., 2016).

Bacterial two hybrid (BACTH) assay - To investigate possible protein-protein interactions between different enzymes of the c-di-AMP regulatory network, a BACTH assay was employed. Therefore, E. coli BTH101 was transformed with the plasmids pBP232-pBP235 (cdaA), pBP224-pBP227 (cdaR), pBP359-pBP362 (glmM), pBP269-pBP272 (pdeA), pBP273-pBP276 (pgpH), pBP277-pBP280 (kimA) and pGP976-pGP979 $\left(r n y^{B s u}\right)$, which all are derivatives of plasmids pUT18, pUT18C, p25-N and pKT25. pUT18C-zip and pKT25-zip served as controls. E. coli was co-transformed with the plasmids in combinations that always a plasmid harboring the T18 and the T25 domain of the CAMP adenylate cyclase were together. As described in more detail elsewhere (Claessen et al., 2008; Karimova et al., 1998), $5 \mu$ l of bacteria were drop spotted on LB medium with kanamycin, ampicillin, streptomycin, $1 \mathrm{mM}$ IPTG and X-Gal for $24-48 \mathrm{~h}$ at $30^{\circ} \mathrm{C}$.

Impact of GImM, CdaR and variants on CdaA activity in E. coli - Plasmid pBP384 (pWH844-kimA) allows the IPTG dependent expression of the L. monocytogenes potassium transporter KimA (see chapter 3). E. coli LB2003 was co transformed with the plasmid together with the pBAD33-based and plasmids pBP370 (cdaA) or pBP373 (cdaA*) that allow the L-arabinose-inducible expression of the L. monocytogenes DAC CdaA or an inactive mutant CdaA* (D171N; Quintana et al., 2019; Rosenberg et al., 2015). This strain is deficient for potassium transport due to deletions in genes encoding for the three major potassium uptake systems of $E$. coli and is only viable in medium with sufficient potassium or if a potassium transporter is expressed in it (Stumpe \& Bakker, 1997; see chapter 3 ). The plasmids PBP370 and pBP373 served hereby as controls for c-di-AMP production and no c-di-AMP production. In the same manner, E. coli LB2003 was co-transformed with the plasmids pBP387 (cdaA-cdaR), pBP388 (cdaA-cdaR-glmM), pBP389 (cdaA-glmM), pBP260 (cdaA-cdaR aa 34-452; $\triangle \mathrm{TM}$ ), pBP261 (cdaA-cdaR aa 1-320; $\triangle \mathrm{YbbR} 4$ ), pBP262 (cdaA-cdaR aa 1230; $\triangle$ YbbR 3-4), pBP263 (cdaA-cdaR aa 1-130; $\triangle$ YbbR 2-4) or pBP264 (cdaA-cdaR aa 1-33; $\triangle T$ TM) together with pBP384, respectively. For growth of LB2003, the $\mathrm{NaCl}$ was replaced with $1 \%(\mathrm{w} / \mathrm{v})$ $\mathrm{KCl}$ in the LB medium to allow growth. Strains were cultivated in $\mathrm{M} 9$ minimal medium with $0.35 \mathrm{mM} \mathrm{KCl}, 50 \mu \mathrm{M}$ IPTG and $0.005 \%(w / v)$ L-arabinose in an Epoch2 Multiwell reader, equipped with the Gen5 software (02.09.2001; BioTek Instruments), as described in more detail elsewhere (see chapter 3 ). Growth rates of the exponential phases were calculated to depict the impact of the different constructs on CdaA activity and therefore also the potassium import via the c-di-AMP-regulated KimA transporter.

\section{Results}

CdaR is membrane-localized and involved in adaptation to osmotic stress - As previously reported, a L. monocytogenes $\triangle c d a R$ (Imo2119) mutant shows elevated intracellular c-di-AMP concentrations, while expression and localization of the DAC CdaA - with which CdaR interacts - is not influenced (Rismondo et al., 2016). CdaR has therefore been postulated to modulate CdaA activity by unknown environmental stimuli. To identify the triggers that are sensed by the CdaR protein, a phenotype microarray was employed. The Biolog PM plates PM1-10 and PM13B were used to investigate the influence of different carbon sources, nitrogen sources, antibiotics, $\mathrm{pH}$ and osmolytes on the metabolic activity of the L. monocytogenes wild type (wt) and the $\Delta c d a R$ mutant (Bochner et al., 2001). The $\Delta c d a R$ mutant showed an altered metabolic activity with some carbon and nitrogen sources such as $\alpha$-D-lactose or L-phenylalanine, respectively and with di- or tri-peptides - especially if they contain aromatic amino acids (Fig. S2.1), but most strikingly was the 
altered metabolic activity on plate PM9, that contains different osmolytes. The $\Delta c d a R$ mutant shows an altered metabolic activity if challenged with osmolytes, such as increasing amounts of $\mathrm{NaCl}$ (Fig. 2.1, A). To test whether the changes in metabolic activity of a $\Delta c d a R$ mutant also reflect in a growth phenotype, the mutant and the L. monocytogenes wt were grown in $\mathrm{BHI}$ medium with increasing concentrations of the osmolytes $\mathrm{NaCl}, \mathrm{KCl}$ or sorbitol. As shown in Fig. 2.1, B. The altered metabolic activity on plate $\mathrm{pM} 8$, containing different osmolytes, was most remarkable. The $\triangle c d a R$ mutant shows a decreased growth rate in the presence of osmolytes, independent of the nature of the osmolyte (ionic, non-ionic). Since $\mathrm{CdaA}$ and $\mathrm{CdaR}$ showed protein-protein interaction, we also tested if CdaR is like CdaA membrane-localized and if both are expressed in minimal medium, a condition under which the $c d a A$ gene is non-essential (Whiteley et al., 2015, Whiteley et al., 2017).

A

\begin{tabular}{|c|c|c|c|c|c|c|c|c|c|c|c|}
\hline \multicolumn{12}{|c|}{$\mathrm{NaCl}$} \\
\hline $\begin{array}{c}0.17 \mathrm{M} \\
(1 \%)\end{array}$ & $\begin{array}{c}0.34 \mathrm{M} \\
(2 \%)\end{array}$ & $\begin{array}{c}0.51 \mathrm{M} \\
(3 \%)\end{array}$ & $\begin{array}{c}0.68 \mathrm{M} \\
(4 \%)\end{array}$ & $\begin{array}{c}0.86 \mathrm{M} \\
(5 \%)\end{array}$ & $\begin{array}{l}0.94 \mathrm{M} \\
(5.5 \%)\end{array}$ & $\begin{array}{c}1.03 \mathrm{M} \\
(6 \%)\end{array}$ & $\begin{array}{l}1.11 \mathrm{M} \\
(6.5 \%)\end{array}$ & $\begin{array}{c}1.2 \mathrm{M} \\
(7 \%)\end{array}$ & $\begin{array}{c}1.37 \mathrm{M} \\
(8 \%)\end{array}$ & $\begin{array}{c}1.54 \mathrm{M} \\
(9 \%)\end{array}$ & $\begin{array}{c}1.71 \mathrm{M} \\
(10 \%)\end{array}$ \\
\hline & & $\longrightarrow$ & 1 & & 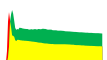 & & & r & & & \\
\hline
\end{tabular}

wt vs. $\triangle c d a R$

$\mathrm{B}$

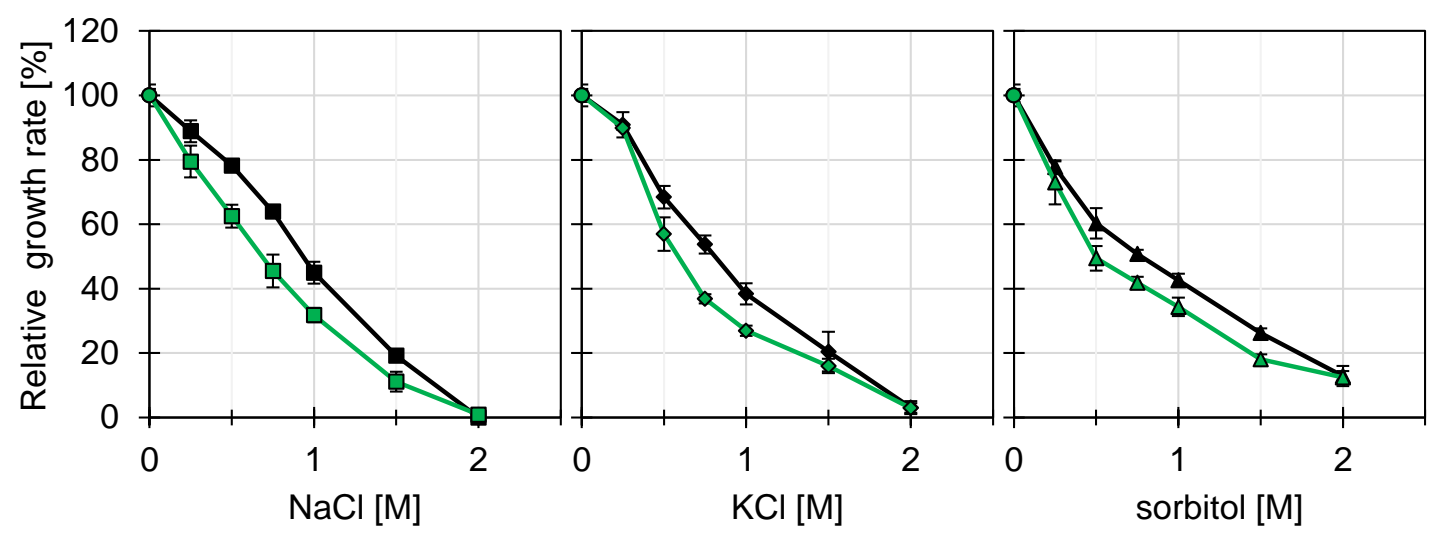

Fig. 2.1 CdaR is important for osmotic adaptation. (A) Phenotype Microarray (PM) of plate PM9 wells A1-A12 of the L. monocytogenes wild type (wt) vs. $\triangle c d a R$ mutant with increasing concentrations of $\mathrm{NaCl}$, shows altered metabolic activity of the $\triangle c d a R$ mutant. (B) A $\Delta c d a R$ mutant shows a decreased growth rate if grown in the presence of osmolytes. The wt and $\triangle c d a R$ mutant were grown in $\mathrm{BHI}$ medium with increasing concentrations of either $\mathrm{NaCl}, \mathrm{KCl}$ or sorbitol. The relative growth rates of exponentially growing bacteria are plotted against the different osmolyte concentrations. Means of biological triplicates and the standard derivations are shown. vs., versus.

The wt, a $\triangle c d a A$ (Imo2120) mutant and a $\triangle c d a R$ mutant were grown in LSM medium. Exponentially growing cells were harvested and lysed. Proteins of whole cell extracts, as well as cytosolic and membrane fractions were separated by SDS-PAGE and protein expression and localization of CdaA, CdaR and the cytosolic transcription factor PrfA (Lmo0200, Mengaud et al., 1991) were investigated by Western blotting, using specific polyclonal antibodies to detect the respective proteins. PrfA is found in the cytosolic fraction and both CdaA and CdaR are enriched in the membrane fraction (Fig. 2.2). CdaR does not impact CdaA expression or localization, as previously reported (Rismondo et al., 2016) and neither CdaA, nor CdaR have an influence on PrfA expression or localization. Furthermore, $\mathrm{CdaR}$ is similar as $\mathrm{CdaA}$ enriched in the membrane fraction, but in contrast to $\mathrm{CdaA}$, the expression of $\mathrm{CdaR}$ seems to be decreased in the $\Delta c d a A$ mutant, probably due to 
secondary effects of the truncated polycistronic cdaA-cdaR mRNA, but once more, localization is not influenced.

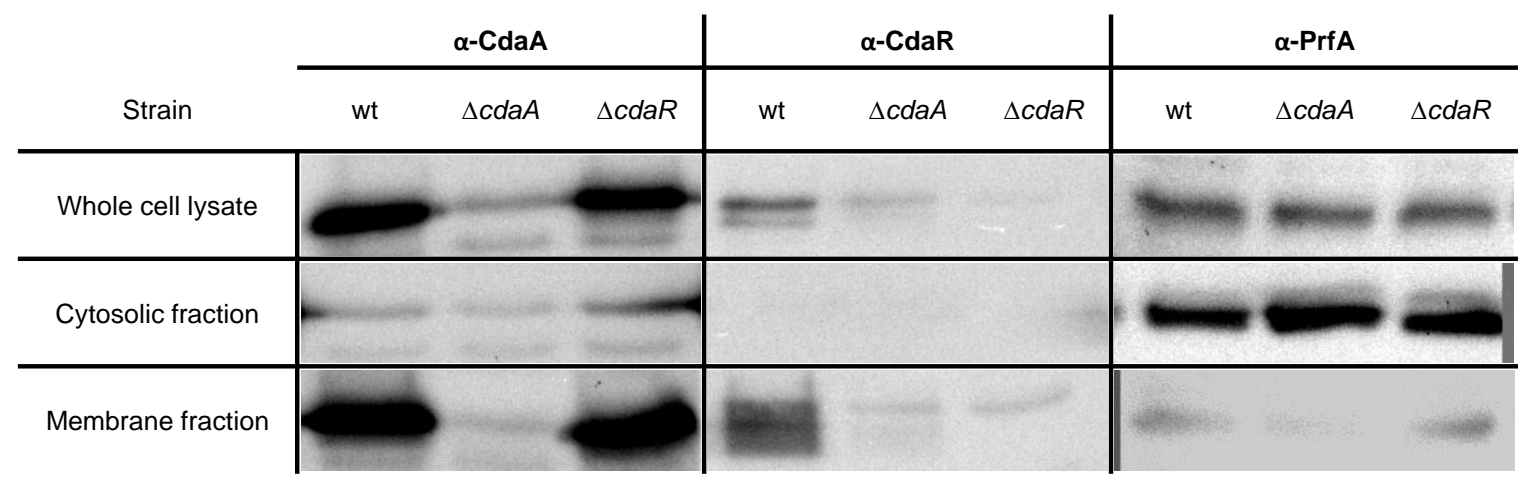

Fig. 2.2 Synthesis and localization of the DAC CdaA and the effector protein CdaR. Expression of $c d a A, c d a R$ and prfA in the L. monocytogenes wild type (wt), a $\triangle c d a A$ mutant and a $\triangle c d a R$ mutant. Bacteria were grown in LSM and expression and localization of the different proteins was investigated for exponential growing cells. Cytosolic and membrane proteins were separated by centrifugation. $10 \mu \mathrm{g}$ of the different protein fractions were separated by SDS-PAGE (12\%) and analysed by Western blotting using CdaA, CdaR or PrfA polyclonal antibodies, respectively.

The YbbR domains are surface exposed - CdaR impacts both, intracellular c-di-AMP concentration and metabolic activity of L. monocytogenes. It is therefore tempting to hypothesis that CdaR senses changes in osmolarity and modulates $\mathrm{CdaA}$ activity accordingly. The CdaR protein consists of an N-terminal TM domain and four YbbR domains of unknown function (Fig. 2.3, A). It has been shown previously that the membrane domain of both, CdaA and CdaR are important for their direct protein-protein interaction (Rismondo et al., 2016), but it is unclear in which part of the cell the YbbR domains are localized and therefore also the signal that might be sensed by them. To analyze the orientation of the CdaR protein in the cell envelope, fusion proteins with C-terminal alkaline phosphatase PhoA and the $\alpha$-fragment of the $\beta$-galactosidase LacZ were constructed using the pKTop vector (Karimova et al., 2009). E. coli DH5 $\alpha$ was transformed with the pKTop empty vector and derivatives for the expression of $\mathrm{CdaR}, \mathrm{CdaR}$ without the $\mathrm{N}$-terminal TM domain ( $\triangle T \mathrm{TM}$, aa 34-452), CdaR without the YbbR domains ( $\triangle \mathrm{YbbR}$, aa 1-33), PrfA as a control for a cytosolic protein and PrkA (Lmo1820, Lima et al., 2011) as a control for a membrane protein. Both, E. coli harboring the pKTop empty vector or the prfA containing derivative show a red coloration on indicator plates and therefore high $\beta$-galactosidase activity and a cytosolic localization of the $C$-terminal part, containing the PhoA and LacZ fusion proteins (Fig. 2.3, B). In contrast, E. coli expressing the PrkA fusion protein show a blue coloration, indicating high alkaline phosphatase activity and therefore a periplasmic localization of the $\mathrm{C}$-terminal PhoA and LacZ fusion proteins. The same is true for full length $\mathrm{CdaR}$, demonstrating an outward localization of the $\mathrm{C}$-terminal YbbR domains. E. coli expressing the $c d a R \Delta T M$ and $\triangle \mathrm{YbbR}$ domain constructs show red and blue colored colonies, respectively, demonstrating that the $\mathrm{N}$-terminal TM domain is both, necessary and sufficient for correct localization of the C-terminal fusion part. To further assess the localization of the YbbR domains and the role of the TM domain for the localization of the CdaR protein, enzymatic assays to determine the alkaline phosphatase and $\beta$-galactosidase activity of the different constructs were performed (Fig. 2.3, C). The enzymatic assays confirm the phenotype of the E. coli plate assay, CdaR is membrane localized in dependency of the TM domain and the YbbR domains are surface exposed. 
A

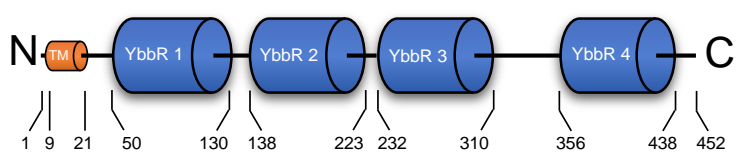

B
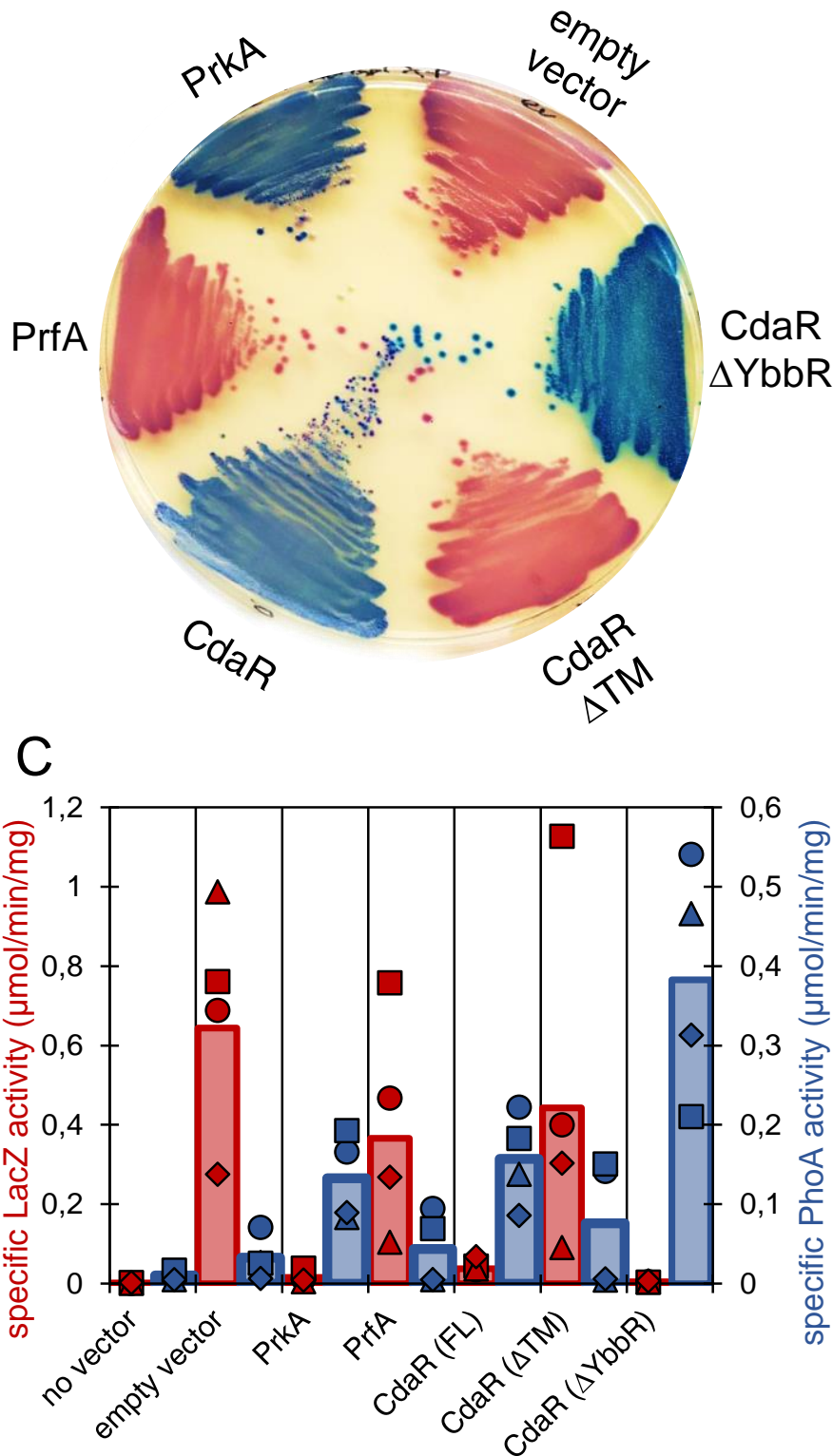

Fig. 2.3 CdaR topology analysis. (A) Domain architecture of the CdaR protein. CdaR consists of four YbbR domains and an $\mathrm{N}$-terminal transmembrane (TM) domain. (B) Determination of the topology of CdaR. E. coli $\mathrm{DH} 5 \alpha$ cells expressing CdaR, CdaR $\triangle T M$ (aa 34-452), CdaR $\triangle$ YbbR (aa 1-33), PrfA (cytosolic control), or PrkA (membrane control) with PhoA and the LacZ alpha fragment fused to the C-terminus or harboring the pKTop empty vector were plated on LB plates with Red-Gal (for $\beta$-galactosidase activity) and X-Pho (for phosphatase activity). Blue colored colonies (phosphatase activity) indicates a periplasmic localization of the C-terminus, while a red coloration ( $\beta$-galactosidase activity) indicates a cytosolic localization. (C) Quantification of PhoA and LacZ activities of protein extracts of E. coli $\mathrm{DH} 5 \alpha$ and $\mathrm{DH} 5 \alpha$ harboring the same fusion proteins as described above. Cells were grown in LB medium and logarithmic growing bacteria were harvested. Cells were lysed and alkaline phosphatase and $\beta$-galactosidase activity of whole cell lysates determined using pNPP and ONPG as substrates, respectively. Specific enzymatic activities of four biological replicates are shown.

CdaR is important for survival of $\mathrm{L}$. monocytogenes upon continuous exposure to increased osmolarity - To further assess the role of CdaR, its membrane localization and the role of the YbbR domains on the ability of $L$. monocytogenes to adapt to osmotic stress, several mutants were constructed. Using the integrative pIMK3 vector that allows IPTG-dependent expression, L. monocytogenes strains were constructed, in the background of the cdaR deletion, to express CdaR without its membrane localization ( $\triangle T \mathrm{TM}$, aa 32-452) and consecutive truncations of the YbbR domains ( $\triangle \mathrm{YbbR} 4$, aa 1-320; $\Delta \mathrm{YbbR} 3-4$, aa 1-230; $\Delta \mathrm{YbbR} 2-4$, aa 1-130; $\Delta \mathrm{YbbR} 1-4$, aa 1-33). As controls the pIMK3 empty vector (ev) was integrated into the EGD-e wt chromosome and into that of the $\triangle c d a R$ mutant and investigated together with a CdaR full-length expressing complementation 
strain (Rismondo et al., 2016). The strains were cultivated in LSM medium and ten-fold serial dilutions drop spotted on LSM agar plates with or without addition of osmolytes. The $\Delta c d a R$ mutant and to a lesser extend the $\triangle c d a R$ mutant expressing CdaR $\Delta T M$ showed slightly decreased growth if challenged with osmolytes (data not shown). After prolonged incubation the $\Delta c d a R$ mutant starts to lyse and suppressor mutants appear (to a lesser extend also in the wt background; Fig. 2.4). In contrast, consecutive truncations of the YbbR domains led to more robust growth of L. monocytogenes. The phenotypes were equally for $\mathrm{NaCl}$ and $\mathrm{KCl}$ as osmolytes and less pronounced with sorbitol (data not shown). Two suppressor mutants of the wt strain, isolated from LSM plates supplemented with $0.5 \mathrm{M}$ sorbitol and 5 suppressor mutants in the $\Delta c d a R$ pIMK3 background from LSM plates supplemented with $0.25 \mathrm{M} \mathrm{NaCl}$ (three) or $0.25 \mathrm{M} \mathrm{KCl}$ (two) were isolated and subjected to WGS. Interestingly, mutations were found in the genes $r b s U$ and $r b s V$ that are involved in the regulatory network leading to inactivation of the stress sigma factor SigB and a mutation was found directly in the $\operatorname{sig} B$ gene. Further mutations were identified in pdeA (silent mutation), Imo1515, encoding a homolog to $B$. subtilis CymR that is a pleiotropic regulator of sulfur metabolism and in Imo1432 that encodes for a protein of unknown function that was also identified in a transposon study searching for salt sensitive L. monocytogenes mutants (Evens et al., 2006; Gardan et al., 2003; see Tab. 2.1).

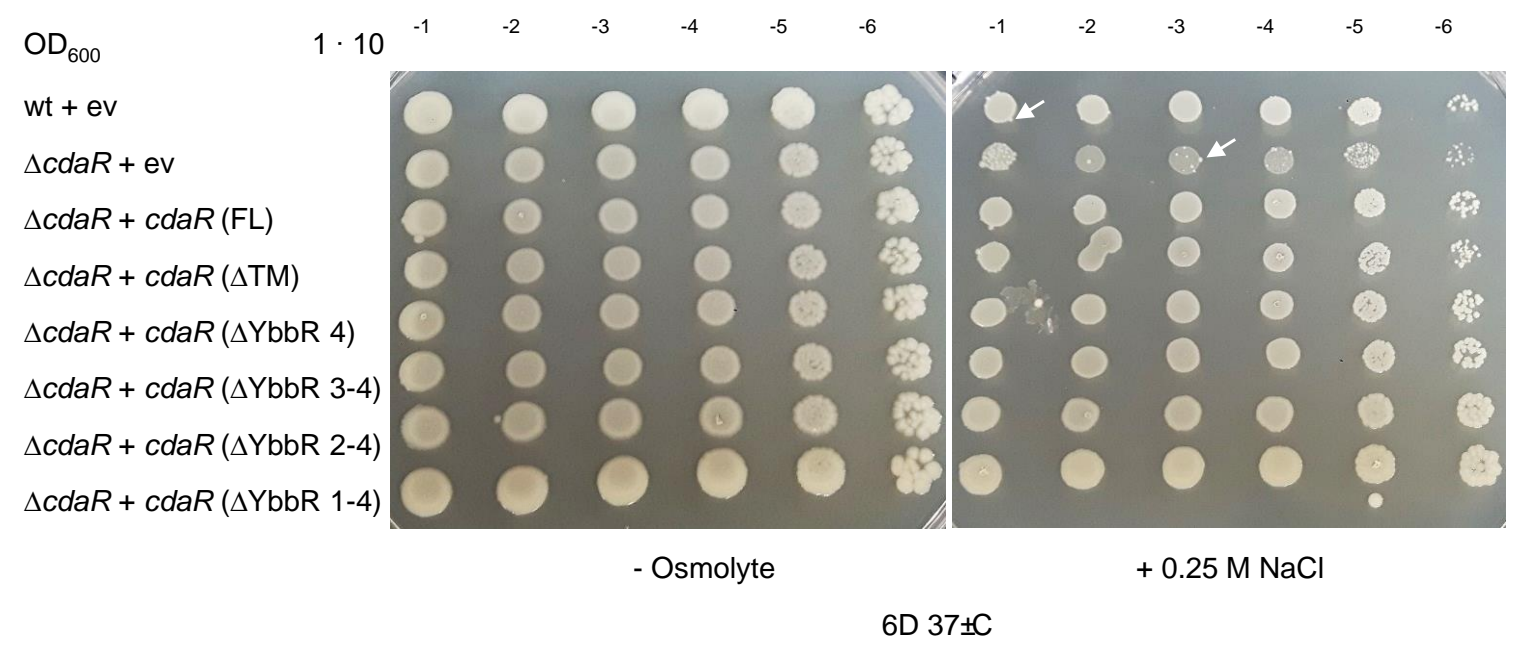

Fig. 2.4 Impact of CdaR, its membrane domain and the YbbR domains on growth under increased osmolarity. The L. monocytogenes wild type (wt) harboring the pIMK3 empty vector (ev), the $\Delta c d a R$ mutant harboring the ev or pIMK3 derivatives for the expression of the full length (FL) CdaR protein, $\mathrm{CdaR}$ without the transmembrane domain ( $\triangle \mathrm{TM}$, aa 32-452) or consecutive truncations of the YbbR domains ( $\triangle \mathrm{YbbR} 4$, aa 1-320; $\Delta \mathrm{YbbR} 3-4$, aa 1-230; $\Delta \mathrm{YbbR} 2-4$, aa 1-130; $\triangle$ YbbR 1-4, aa 1-33) were analyzed for their growth and long term adaptation under increased osmolarity. Bacteria were grown in LSM and ten-fold serial dilutions plated on LSM plates with or without $0.25 \mathrm{M} \mathrm{NaCl}$ at $37^{\circ} \mathrm{C}$ growth was observed every 24 hours. Image shows plate after six days (6D). Arrows indicate appearance of suppressor mutants in the $\triangle c d a R$ mutant and to a lesser extend also in the wt background.

Impact of CdaR on the intracellular c-di-AMP concentration upon osmotic stress - To assess the impact of CdaR, its membrane localization and the YbbR domains on the concentration of c-di-AMP inside L. monocytogenes cells, the wt and the $\triangle c d a R$ mutant (harboring the pIMK3 ev) and $\triangle c d a R$ mutants harboring pIMK3 derivatives for the expression of full-length $C$ daR and CdaR without the membrane localization $(\triangle T M)$ or without the YbbR domains $(\triangle \mathrm{YbbR})$ were investigated. Bacteria were grown in LSM media and the bacteria stressed by the addition of $0.25 \mathrm{M}$ $\mathrm{NaCl}$. Samples before and $25 \mathrm{~min}$ after osmotic challenge (as well as control samples without addition of osmolytes) were analyzed. In all cases, c-di-AMP concentrations decreased after osmotic stress. In the $\triangle c d a R$ mutant and the strain expressing CdaR without the TM domain, c-di-AMP concentrations were lower than in the wild type and c-di-AMP changes less pronounced (Fig. 2.5). 
In contrast, the complementation strain expressing the $c d a R$ gene from an IPTG-dependent promoter, showed increased c-di-AMP concentrations and a greater range of change. Interestingly, the $\triangle c d a R$ complemented with CdaR without the YbbR domains, showed very low c-di-AMP concentrations.

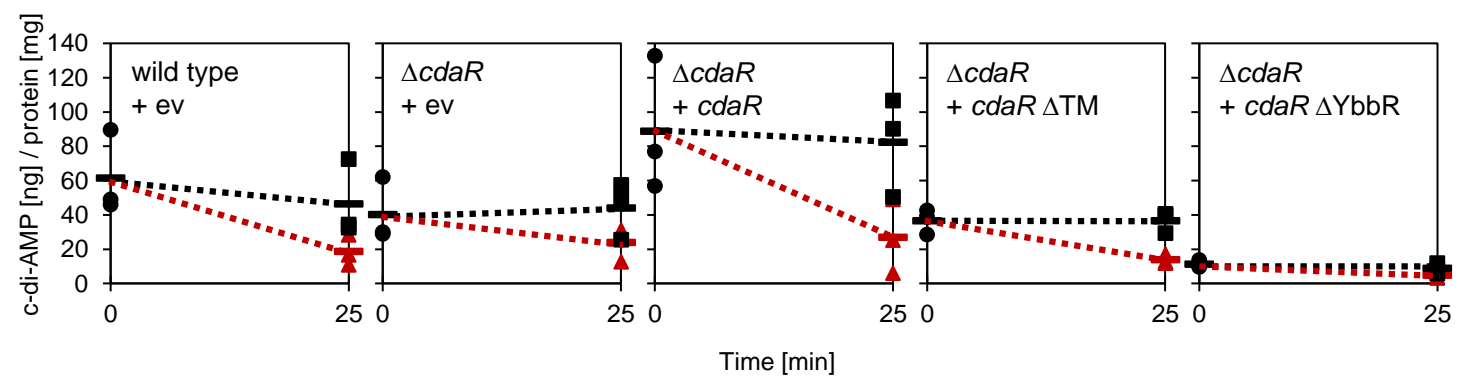

Fig. 2.5 Roles of CdaR, its transmembrane domain and the YbbR domains on intracellular c-di-AMP concentrations during osmotic stress. L. monocytogenes wild type (wt), harboring the pIMK3 empty vector (ev), the $\Delta c d a R$ mutant harboring the ev or derivatives for the expression of full-length CdaR, CdaR without the transmembrane domain $(\triangle T M$, aa 32-452) or without the YbbR domains ( $\triangle \mathrm{YbbR}$, aa 1-33) was grown in LSM at $37^{\circ} \mathrm{C}$. Samples of logarithmic growing cells were taken ( $\mathrm{t}_{0}$; black circles) and the cultures split. Half was subjected to osmotic stress, by the addition of $0.25 \mathrm{M}$ $\mathrm{NaCl}$, while the other half was treated with the same volume of LSM without salt addition. After 25 minutes, additional samples ( $\mathrm{t}_{25}$ ) of osmotic stressed (red triangles) and non-stressed cells (black squares) were taken. Intracellular c-di-AMP levels were determined and normalized to the cellular protein amounts. Data of biological triplicates are shown.

Analysis of protein-protein interactions of components involved in c-di-AMP signaling - To investigate interactions between different parts of the c-di-AMP regulatory network, the DAC CdaA and its two effector proteins ( $\mathrm{CdaR}$ and the phosphoglucosamine mutase GImM), the two PDEs PdeA and $\mathrm{PgpH}$ and the c-di-AMP regulated potassium transporter KimA (see chapter 3 ) bacterial two hybrid (BACTH) assays were employed (Karimova et al., 1998). The leucine zipper protein Zip served as a control and additionally the $5^{\prime} \rightarrow 3^{\prime}$-exoribonuclease RNase $Y$ from $B$. subtilis was employed as an additional control (Commichau et al., 2009). The BACTH analysis (Fig. 2.6) shows interactions between CdaA and CdaR, GlmM, PdeA and PgpH and between CdaR and CdaA, CdaR, $\mathrm{PdeA}, \mathrm{PgpH}$ and KimA, demonstrating protein-protein interactions between synthesis and degradation machinery. Strong self-interaction of the GImM protein, the PdeA and the PgpH PDEs is seen, additionally. Interestingly, also strong interactions between the two PDEs. Furthermore, interactions between the PDEs and KimA, as well as self-interactions of KimA can be seen. None of the proteins interacts with the Zip control, but the second control protein, RNase $Y$, seems to interact with every protein, including the cytosolic GImM and the Zip control.

CdaR and GImM influence CdaA activity - Two investigate the impact of CdaR and CdaR variants lacking membrane localization or YbbR domains and the influence of the GlmM enzyme on CdaA activity, a previously established reporter system was used (see chapter 3 ). Hence, the potassium transporter deficient E. coli strain LB2003 (Stumpe and Bakker, 1997) harboring the pWH844based plasmid for expression of the c-di-AMP inhibited KimA potassium transporter was used. The strain was transformed with pBAD33 plasmids for the expression of CdaA or an inactive mutant (D171N; Rosenberg et al., 2015) as controls for c-di-AMP synthesis and non-synthesis (Quintana et al., 2019). Synthesis of c-di-AMP leads to inhibition of KimA, a decrease in potassium import and therefore reduced growth of the bacteria. To investigate the influence of the effector proteins on CdaA activity, independent of other proteins of the c-di-AMP metabolism, the genes encoding the CdaR protein, the GImM protein or both, were cloned together with CdaA into the pBAD33 vector. In a similar approach, co-expression vectors of $\mathrm{CdaA}$ together with CdaR $\triangle \mathrm{TM}$ (aa 34-452) or CdaR with consecutive truncations of the YbbR domains ( $\triangle \mathrm{YbbR} 4$, aa1-320; $\Delta \mathrm{YbbR} 3-4$, aa 1- 
230; $\triangle \mathrm{YbbR} 2-4$, aa 1-130; $\triangle \mathrm{YbbR} 1-4$, aa 1-33) were constructed. The cells were grown in $\mathrm{M9}$ minimal medium with a defined potassium concentration of $0.35 \mathrm{mM}\left(\mathrm{K}_{\mathrm{M}}\right.$ of KimA), expression of the CdaA operons was induced and growth monitored. High activity of CdaA leads to a decrease in growth, while inhibition of CdaA leads to an increase in growth.
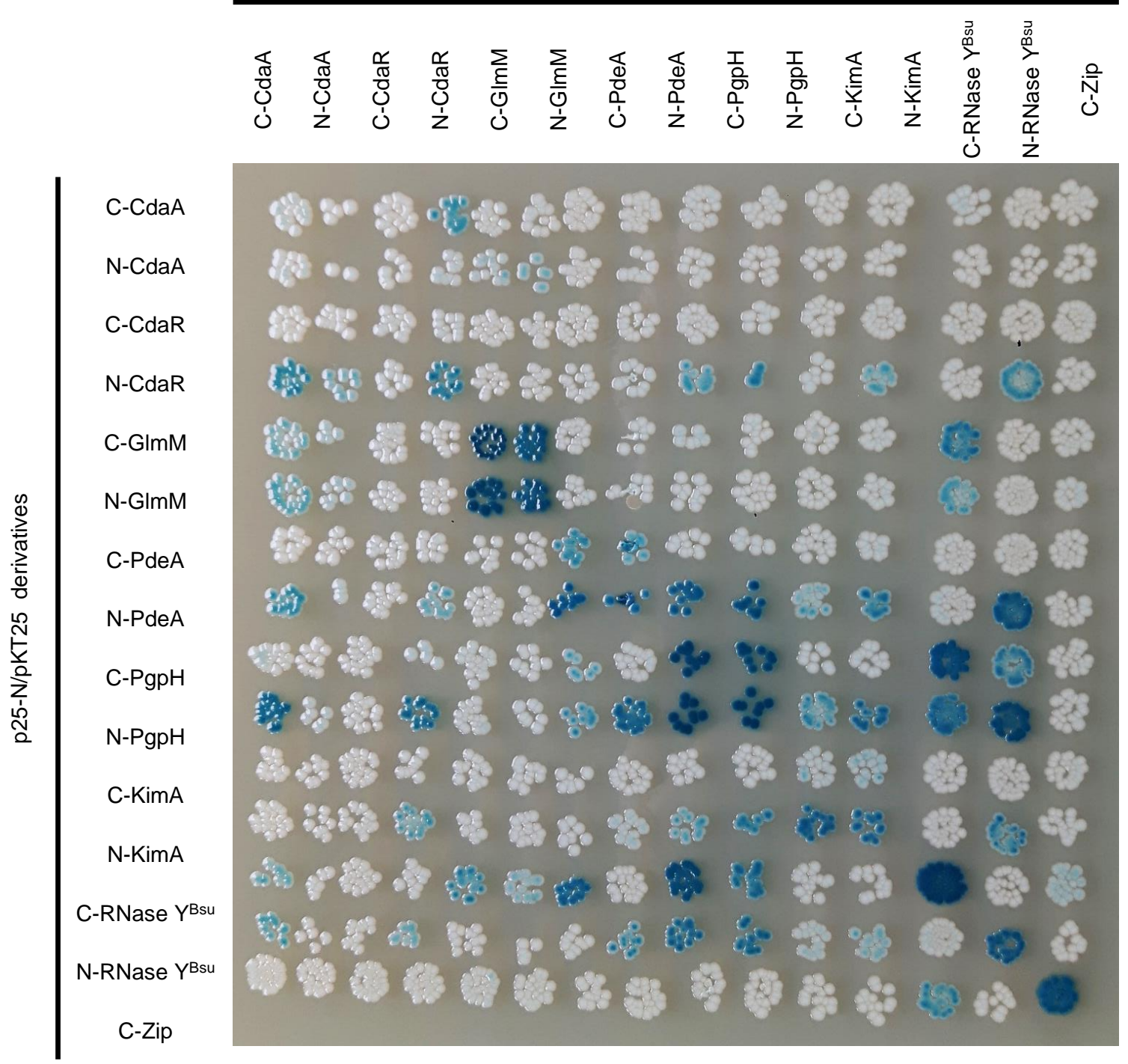

Fig. 2.6 Analysis of protein-protein interactions between proteins of the c-di-AMP synthesis and degradation machinery and the KimA potassium transporter. E. coli BTH101 cells were co-transformed with pUT18/pUT18C derivatives and p25-N/pKT25 derivatives for the expression of fusion proteins of CdaA, CdaR, GImM, PdeA, PgpH or KimA from L. monocytogenes, as indicated. The leucine zipper protein (Zip) served as a control. As an additional control the RNase $Y$ protein from $B$. subtilis was included. Cells were plated on selective LB agar plates supplemented with 1 mM IPTG for induction of protein expression and $100 \mu \mathrm{g} / \mathrm{ml} \mathrm{X-Gal} \mathrm{as} \mathrm{a} \mathrm{colorimetric} \mathrm{substrate} \mathrm{for} \mathrm{visualization} \mathrm{of} \beta$-galactosidase activity and incubated at $30^{\circ} \mathrm{C}$ for 36 hours.

Growth rates of the different strains were determined and as shown in Fig. 2.7, CdaR and to a lesser extend GImM restore the growth rate, compared to the strain synthesizing CdaA alone. If both are present, growth is also substantially restored. Expression of CdaR lacking the TM domain shows the most similar growth rate compared to CdaA alone, indicating that membrane localization is important for CdaR to act on CdaA. Interestingly, the truncations of the YbbR domains have no impact on the growth rate, compared to the full-length variant, as long as one domain is still 
present. The strain expressing only the TM domain of CdaR shows an intermediate phenotype, indicating that the TM domain alone is able to influence CdaA activity to some degree.

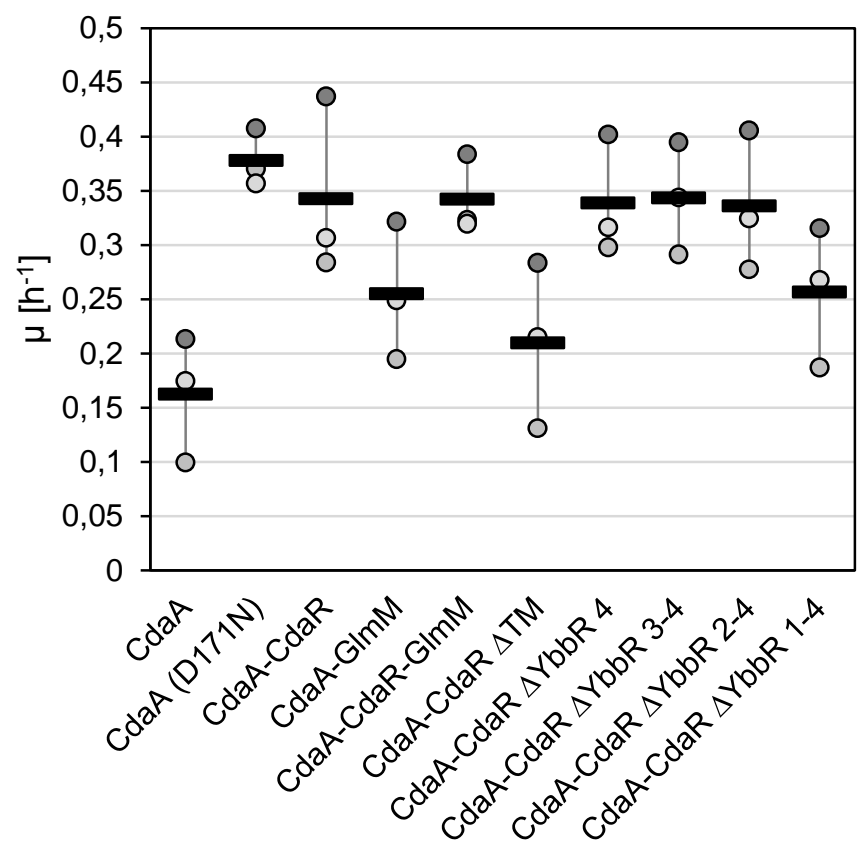

Fig. 2.7 Effect of CdaA and the effector proteins GImM and CdaR growth of E. coli expressing the c-di-AMP regulated KimA potassium transporter. The potassium transporter deficient E. coli strain LB2003 was transformed with a plasmid for the expression of the c-di-AMP-inhibited potassium transporter KimA from L. monocytogenes. Using pBAD33 derivatives, $\mathrm{CdaA}$, an inactive mutant (D171N), CdaA together with either CdaR, GImM or both, or CdaA together with $\mathrm{CdaR}$ variants ( $\triangle \mathrm{TM}$, aa 34-452; $\triangle \mathrm{YbbR} 4$, aa 1-320; $\triangle$ YbbR 3-4, aa 1-230; $\Delta$ YbbR 2-4, aa 1-130; $\triangle$ YbbR 1-4, aa 1-33) were co-expressed together with KimA. Cells were grown in M9 minimal medium with a defined potassium concentration of $0.35 \mathrm{mM}\left(\mathrm{K}_{\mathrm{M}}\right.$ of $\left.\mathrm{KimA}\right)$, $50 \mu \mathrm{M}$ IPTG for KimA induction and $0.005 \%$ $(w / v)$ L-arabinose for induction of the different CdaA operons. Growth rates $(\mu)$ of three biological replicates and means (black bars) are shown.

\section{Discussion}

CdaR impacts osmoregulation in L. monocytogenes - It has been previously reported that CdaR impacts the intracellular c-di-AMP concentration for different bacteria, such as B. subtilis, L. monocytogenes or S. aureus (Bowman et al., 2016; Mehne et al., 2013; Rismondo et al., 2016; Zhu et al., 2016). Interestingly, in $S$. aureus a $\Delta c d a R$ ( $y b b R$ ) mutant was also more susceptible to acidic stress and required suppressor mutations that increased the c-di-AMP concentration, among others in the DAC encoding gene dacA (Bowman et al., 2016). Due to the genetic conservation of $c d a R$ with the CdaA-type encoding genes and direct protein-protein interactions and the influence of CdaR on CdaA activity, CdaR has been proposed to be a modulator of CdaR activity with the YbbR domains of unknown function as sensory domains (Mehne et al., 2013; Rismondo et al., 2016). Here, we show that the sensing of changes in osmolarity might be the trigger that CdaR senses to modulate CdaA activity. CdaR influences the metabolic activity of $L$. monocytogenes in the presence of osmolytes and leads to a decreased growth rate (Fig. 2.1).

The outwards localized YbbR domains and the membrane localization are important for the function of CdaR - The next question we asked was whether CdaR is like CdaA also a membrane-bound protein and if the putative sensory YbbR domains are located in the cytoplasm or outside of the cell. Western blot analysis of CdaR expression and localization shows that CdaR is enriched in the membrane fraction. In the $\triangle c d a A$ mutant, $C d a R$ seems to be less abundant than in the wt, but this effect might be due to secondary effects of the truncation of the polycistronic cdaA-cdaR mRNA (Fig. 2.2; Mehne et al., 2013). To address the questions of the localization of the YbbR domains and the role of the N-terminal TM domain (Fig. 2.3, A), the pKTop system, described by Karimova et al., was used (Karimova et al., 2009). One hypothesis was that the YbbR domains might sense the intracellular concentration of osmolytes, such as potassium ions, and confer that information directly to the cytoplasmic DAC domain of CdaA. The YbbR domains share some structural 
similarity with the C-terminal domains of TL5 and L25 ribosomal proteins (Barb et al., 2010; Fedorov et al., 2001; Lu \& Steitz, 2000). Even though the role of those proteins is not yet fully understood, potassium and magnesium ions are crucial for proper function of the ribosome and T25 of E. coli has been crystallized binding RNA and five magnesium ions (Lu \& Steitz, 2000). The second hypothesis was that the YbbR domains are located in the extracellular space and sense either integrity or composition of the bacterial cell wall, interact with other proteins or ligands, sense the extracellular osmolarity or sense turgor changes by self-interaction of the YbbR domains (Rismondo et al., 2016). The sensing of turgor changes would thereby be sensed as changes in the YbbR domain self-interactions through lateral forces when the turgor changes. In this case, the only interaction surface between CdaR and CdaA would be the TM domains. In the past, c-di-AMP and cell wall biosynthesis have been linked by many observations (Kaplan Zeevi et al., 2013; Luo \& Helmann, 2012). Many of the reported phenotypes could however be indirect phenotypes due to the effects of c-di-AMP on osmotic homeostasis (Commichau et al., 2018). The PhoA and LacZ activity assays (Fig. 2.3, B \& C) demonstrate that the TM domain is both, necessary and sufficient for periplasmic localization of the C-terminus in E. coli and the YbbR domains are located the cell surface. Following the closer analysis of the membrane topology of CdaR, we wanted to analyze the effect of localization and presence of the YbbR domains on the ability of L. monocytogenes to adapt to osmotic stress and their role in adapting the c-di-AMP concentration upon osmotic stress. As shown in Fig. 2.4, deletion of cdaR or lack of the TM domain, leads to lysis of bacteria after prolonged growth on LSM plates supplemented with osmolytes. CdaR seems to be important for survival under osmotic stress. Interestingly, truncations of the YbbR domains increased L. monocytogenes capability to thrive under the same conditions. Analyzing the c-di-AMP concentration in these mutants, the $\triangle c d a R$ mutant and the mutant lacking the TM domain showed a decreased c-di-AMP content and a decreased range comparing the changes of osmotic stressed and unstressed cells (Fig. 2.5). In contrast, the complementation mutant, in which the cdaR full-length gene is expressed under a strong IPTG-dependent promoter (Monk et al., 2008), shows elevated c-di-AMP concentration and an increased range between stressed and unstressed cells. This demonstrates the role of CdaR in sensing osmotic changes and conferring those on the intracellular c-di-AMP concentration. The strain expressing CdaR without the TM domain behaved most similar to the $\triangle c d a R$ mutant, while the mutant lacking all four YbbR domains showed in general very low concentrations of c-di-AMP. This demonstrates that the YbbR domains - their proper localization and presence - are crucial for the correct function of CdaR. To isolate potential effects of other parts of the c-di-AMP metabolic network, such as degradation by PDEs, or efflux by multi drug efflux system, the effect of CdaR and CdaR mutants, as well as the GImM enzyme was investigated in E. coli (Kaplan Zeevi et al., 2013; Mehne et al., 2013). Using the growth dependency of an E. coli strain lacking native potassium transporter on the activity of the c-di-AMP regulated potassium transporter KimA from L. monocytogenes as a readout, we investigated the direct effect on the ability of CdaA to produce c-di-AMP as a screening system for DAC function (see chapter 3). The growth phenotypes demonstrate that CdaR and to a lesser extend GImM can inhibit CdaA activity in vivo. While the TM domain for CdaR seems to be important for CdaR function, only one YbbR domain seems to be required for proper function. CdaR consisting only of the TM domain, however, still seems to have some impact on CdaA activity (Fig. 2.7). Intriguingly, the effect of CdaR lacking all YbbR domains is the opposite in E. coli for what we observe in L. monocytogenes (Fig. 2.5). The reason for this remains elusive, but it could either be a consequence of the different structure of the Gram-positive and Gram-negative cell envelope or a possible crosstalk between synthesis and degradation machinery in L. monocytogenes. To investigate this possibility, a BACTH assay was employed to analyze protein-protein interactions between different components of the c-di-AMP metabolic network. The assay demonstrated potential interactions 
between CdaA and CdaR and the PDEs. A possible influence of CdaA or CdaR on PDE activity could be the reason for the phenotype discrepancy between E. coli and L. monocytogenes and should be the subject of future studies. A recent publication by Pham et al. demonstrates the effects of osmotic up- and downshifts on the c-di-AMP concentration of various bacteria, including L. monocytogenes (Pham et al., 2018). They demonstrated that an osmotic downshift, a hypoosmotic shock, leads to a rapid increase of the c-di-AMP concentration, while an osmotic upshift, a hyperosmotic shock, leads to a rapid decrease. As depicted in the model in Fig. 2.8, the regulation of synthesis and degradation have to be coordinated to increase the c-di-AMP concentration for preventing further increase of osmolytes during a hypoosmotic stress and to decrease the c-di-AMP concentration to allow influx of osmolytes during a hyperosmotic stress. The CdaR protein might regulate CdaA activity, as depicted, by interacting with itself via the YbbR domains and with CdaA via the TM domains of the two proteins, acting as a buffer for CdaA self-interaction and therefore activity in a positive or negative manner, depending on the forces in the cell envelope during osmotic driven turgor changes (Fig. 2.8).

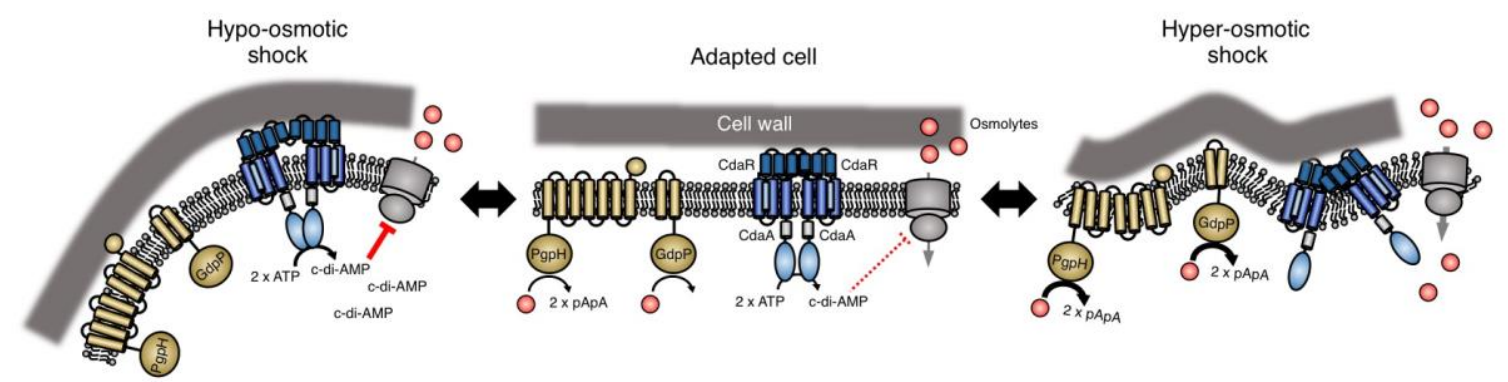

Fig. 2.8 Model depicting the control of c-di-AMP synthesis and degradation in dependence of the external osmolarity. Bacteria that are adapted to the osmotic state in the environment live in an equilibrium of c-di-AMP synthesis and degradation and osmolyte import and export to maintain the cellular turgor. Sudden changes in external osmolarity change this equilibrium. A decrease in external osmolarity, a hypoosmotic shock, leads to rapid influx of water and osmotic swelling. Under these conditions, c-di-AMP has to be synthesized to prevent excess accumulation of osmolytes. Hence, PDE activity has to decreases, while DAC activity has to be increased. The CdaR protein could function as a stabilator of $\mathrm{CdaA}$ interaction. The opposite, a hyperosmotic shock, e.g. rapid efflux of water to dilute an outside excess of osmolytes and shrinkage of cellular volume leads to membrane invaginations. To counteract, c-di-AMP synthesis has to be de- and PDE activity increased. The CdaR protein could function in this context as a buffer to decrease CdaA selfinteraction.

The c-di-AMP synthesis and degradation machineries might build up interacting networks L. monocytogenes $\Delta c d a R$ mutant colonies on minimal media (LSM) agar plates supplemented with osmolytes became translucent after prolonged incubation and suppressor mutants appeared. To a lesser extent, suppressor mutants also appeared in the wt background without changes in colony morphology. Five mutants in the $c d a R$ background and two mutants in the wt background were subsequent subjected to WGS. The majority of mutations were found in the regulatory pathway, regulating activity of the stress sigma factor SigB. There also mutations were found in genes of unknown function, involved in regulation of cysteine metabolism, a silent mutation in the PDE encoding gene $p d e A$, mutations in genes regulating activity of the sigma factor SigB and in the gene sigB itself (see Tab. 2.1). The mutations affecting SigB, either truncated SigB directly or affected genes necessary for activation of SigB, all presumably inactivating SigB. Interestingly, it has been previously reported for $L$. monocytogenes that inactivation of SigB leads to hyperresistance against oxidative stress (Boura et al., 2016). The reason why inactivation of SigB, which should activate expression of genes to cope with various stresses, such as oxidative or osmotic stress, causes an increased resistance of $L$. monocytogenes is fascinating but needs further investigations. It seems, however, be independent of the c-di-AMP metabolism, since similar mutations were 
identified in both, the wt and the $\triangle c d a R$ mutant. To conclude, we could show that $\mathrm{CdaR}$ is important for adaptation to osmotic stress, by regulating CdaA activity. Localization and presence of the outwards and self-interacting YbbR domains (Rismondo et al., 2016) is important for proper CdaR function. Another way for L. monocytogenes to cope with osmotic stress is the inactivation of SigB that leads to increased resistance against osmotic stress. Finally, we showed that components of the synthesis and degradation machinery interact with each other, demonstrating potential regulatory cross-talk inside the c-di-AMP regulatory network.

\section{Acknowledgements}

We are grateful to Jonathan Rosenberg, Lisa Maria Schulz, Sandra Klama and Veronika Lutz for help with some experiments and for Anna-Lena Hagemann and Annette Garbe for technical assistance This work was supported by the grant CO 1139/2-1 from the Deutsche Forschungsgemeinschaft via the Priority Program SPP1879, the Fonds der Chemischen Industrie and the Max-Buchner-Forschungsstiftung (MBFSt-Kennziffer 3381) to FMC. 
Cyclic di-AMP and osmoregulation in Listeria monocytogenes 


\title{
3. Characterization of c-di-AMP-controlled potassium trans- porters of Listeria monocytogenes
}

Johannes Gibhardt, Gregor Hoffmann, Vincent T. Lee, and Fabian M. Commichau

Author contribution:

JG and GH performed the experiments. VTL performed the DRaCALA binding experiments. JG and FMC wrote the manuscript.

\begin{abstract}
Many bacteria and some archaea produce the second messenger cyclic diadenosine monophosphate (c-di-AMP). c-di-AMP was shown to be essential for growth of Firmicutes, including the human pathogen Listeria monocytogenes because it controls the uptake of osmolytes such as glycine betaine and potassium ions. The cellular c-di-AMP levels have to be tightly regulated for optimal growth in environments with changing osmolarities. Here, we have identified and characterized the high- and low-affinity potassium transporters KimA and KtrCD of L. monocytogenes, respectively. We also show that Staphylococcus aureus contains a homolog of KimA, which mediates potassium transport. The KimA and KtrCD transporters from L. monocytogenes are both inhibited by c-di-AMP in vivo. The C-terminal domain of KimA seems to be involved in the c-di-AMP-dependent regulation of the transporter. The nucleotide also binds to the cytoplasmatic regulatory subunit KtrC of the KtrCD potassium transporter in vitro. Thus, many phylogenetically related bacteria use c-di-AMP for controlling the uptake of osmolytes such as potassium, which is essential to adjust the cellular turgor to the environment.
\end{abstract}




\section{Introduction}

Bacteria use complex signal transduction systems to adjust the cellular turgor to the environmental osmolarity (Sleator and Hill, 2002; Wood, 1999; Wood, 2011). Under hyperosmotic growth conditions, potassium ions are imported to prevent water efflux from the cytosol and to increase the cellular turgor (Kempf and Bremer, 1998). The potassium ions are thereupon often replaced by compatible solutes such as glycine betaine and ectoine; osmolytes that do not disturb essential cellular processes (Kempf and Bremer, 1998). Depending on the external osmolarity, the import and export of osmolytes have to be tightly controlled to prevent osmotic swelling and shrinking of the cell, respectively (Commichau et al., 2018; Gundlach et al., 2017; Wood, 1999). Although osmoregulation has been intensively studied, it is still rather unclear how cell sense the environmental osmolarity to adjust the turgor accordingly. The second messenger c-di-AMP, which is produced by specific diadenylate cyclases (DACs), plays a key role in regulating the turgor in Firmicute bacteria because it controls the uptake and export of osmolytes including potassium (see below; Bai et al., 2014; Chin et al., 2015; Corrigan et al., 2013; Gundlach et al., 2017; Huynh et al., 2016; Pham et al., 2018; Schuster et al., 2016; Whiteley et al., 2015; Whiteley et al., 2017; Zeden et al., 2018).

c-di-AMP was discovered during the structural characterization of DNA integrity scanning protein DisA, which is involved in DNA damage response and in controlling sporulation initiation in the Gram-positive bacterium Bacillus subtilis (Bejerano-Sagie et al., 2006; Gándara and Alonso, 2015; Raguse et al., 2017; Valenzuela-García et al.,2018; Witte et al., 2008). DisA is present in sporeforming Firmicutes, in actinobacteria (Corrigan and Gründling, 2013) and in hyperthermophilic bacteria (Witte et al., 2008). While DisA is the only c-di-AMP-producing enzyme in actinobacteria, bacteria like $B$. subtilis also contain the DACs CdaA and CdaS, of which the latter is required for efficient spore germination (Mehne et al., 2013; Commichau et al., 2019). CdaA is attached to the membrane and DisA and CdaS are soluble proteins (Mehne et al., 2013; Rismondo et al., 2016; Witte et al., 2008). CdaA is the most abundant DAC and many prominent apathogenic and pathogenic Gram-positive bacteria, such as Lactococcus lactis, L. monocytogenes, S. aureus, Streptococcus agalactiae, rely only on this DAC for c-di-AMP synthesis (Corrigan and Gründling, 2013). Since c-di-AMP is essential for growth of these bacteria (Devaux et al., 2018; Woodward et al., 2010; Zeden et al., 2018), the DAC CdaA is an interesting target for novel antibiotics.

c-di-AMP is degraded intracellular by specific phosphodiesterases (PDEs), which can be assigned to three different groups (Commichau et al., 2019; Huynh and Woodward, 2016). The GdpP-and $\mathrm{PgpH}$-type PDEs consist of domains that are involved in signaling and enzyme catalysis. Both PDEs are attached to the membrane, suggesting that the enzymes may sense and respond to extracellular cues. The DhhP-type PDEs, which are located in the cytosol, form the third group of c-di-AMPdegrading enzymes (Huynh and Woodward, 2016). Since the DACs and the PDEs determine the cellular c-di-AMP levels that are required for optimal growth in environments with changing osmolarities (Gundlach et al., 2017; Pham et al., 2018), the activities of the enzymes have to be tightly regulated. Recently, it has been observed that the phosphoglucosamine mutase GImM inhibits the DAC CdaA in L. lactis, suggesting a link between c-di-AMP metabolism and cell wall biosynthesis (Zhu et al., 2016). However, the molecular mechanisms by which the DACs, the PDEs and GlmM sense the environmental osmolarity are unknown. Moreover, the sensing mechanisms may vary among the enzymes due to the different domain composition and cellular localization.

One important aspect of understanding c-di-AMP signaling was the identification of targets of c-di-AMP. Several have been identified, so far: c-di-AMP activates the DNA-binding activity of the 
transcription factor DarR in Mycobacterium smegmatis (Zhang et al., 2013). In L. monocytogenes, c-di-AMP inhibits the pyruvate carboxylase PycA (Choi et al.,2017; Sureka et al.,2014). c-di-AMP also binds to the cystathione-beta-synthase domain-containing (CBS) proteins $\mathrm{CbpA}$ and $\mathrm{CbpB}$ and the PII-like signal transduction DarA in this organism (Choi et al.,2015; Sureka et al.,2014). The DarA homologs from B. subtilis and S. aureus have also been structurally and biochemically characterized (Campeotto et al., 2015; Gundlach et al., 2015a; Müller et al., 2015a). While the biological functions of $\mathrm{CbpA}, \mathrm{CbpB}$ and DarA remain to be elucidated, several c-di-AMP targets are involved in the transport of osmolytes such as potassium, glycine betaine and carnitine (Corrigan et al.,2013; Huynh et al.,2016; Schuster et al.,2016; Whiteley et al.,2017; Zeden et al.,2018). c-di-AMP inhibits the KtrCD/KtrCB and CabP-TrkH potassium uptake systems from S. aureus and S. pneumoniae, respectively, by binding to the membrane-associated gating proteins (Bai et al.,2014; Corrigan et al.,2013; Kim et al.,2015). In S. aureus, the synthesis of the KdpFABC potassium transporter, is also inhibited by c-di-AMP (Moscoso et al.,2016). In the same organism, c-di-AMP stimulates the potassium and sodium transporter CpaA (Chin et al.,2015). In B. subtilis, the expression of the ktrAB and kimA genes, encoding the potassium transporters KtrAB and KimA, respectively, is negatively regulated by c-di-AMP (Gundlach et al.,2017). c-di-AMP also controls the uptake of the compatible solutes carnitine in L. monocytogenes and S. aureus (Schuster et al.,2016; Whiteley et al.,2017). Moreover, the DNA-binding transcription factor BusR represses the genes involved in glycine betaine uptake in S. agalactiae and L. lactis depending on the cellular c-di-AMP concentration (Devaux et al.,2018; Pham et al.,2018).

We are interested in the c-di-AMP-dependent control of osmolyte homeostasis in the food-borne pathogen L. monocytogenes (Rolhion and Cossart, 2017). The ability of L. monocytogenes to thrive under adverse conditions including high osmolarity depends on the c-di-AMP-dependent control of osmolyte transport such as carnitine (Whiteley et al.,2017). However, the involvement of c-di-AMP in potassium uptake or homeostasis in L. monocytogenes has remained elusive. Here, we show that the L. monocytogenes KimA (Lmo2130) and KtrCD (Lmo1023 and Lmo0993) proteins are high- and low-affinity potassium transporters, respectively. We also show that the transporters are inhibited by c-di-AMP and that unregulated activity leads to rapid osmotic swelling. The interaction between c-di-AMP and KtrC was also confirmed in vitro. Moreover, the C-terminal domain of KimA is important for the c-di-AMP-dependent regulation of potassium uptake.

\section{Experimental procedures}

Bacterial strains and growth conditions - The bacterial strains are listed in Tab. 3.1. The Escherichia coli strains XL1-Blue (Stratagene), Rosetta (DE3) (Novagen) and T7 Express $1^{q}$ (NEB) were used for cloning and protein overproduction. E. coli was grown in LB medium and transformants were selected on LB plates ( $15 \mathrm{~g} / \mathrm{l}$ Bacto agar (Difco)) containing kanamycin $(50 \mathrm{\mu g} / \mathrm{ml})$, ampicillin, carbenicillin $(100 \mu \mathrm{g} / \mathrm{ml})$ or chloramphenicol $(30 \mu \mathrm{g} / \mathrm{ml})$. The L. monocytogenes wild type strain EGD-e (laboratory strain collection) was cultivated in BHI medium (Sigma-Aldrich) The B. subtilis wild type strain 168 (laboratory strain collection) was cultivated in LB medium. Potassium transporter deficient $E$. coli strains LB650 and LB2003 were cultivated in LB-K medium ( $\mathrm{NaCl}$ substituted by $1 \% \mathrm{KCl}(w / v))$ (Stumpe \& Bakker, 1997). M9 medium was used for $E$. coli growth experiments with the following composition: $37.85 \mathrm{mM} \mathrm{Na}_{2} \mathrm{HPO}_{4}, 22.05 \mathrm{mM} \mathrm{KH}_{2} \mathrm{PO}_{4}, 18.75 \mathrm{mM} \mathrm{NH}_{4} \mathrm{Cl}, 1 \mathrm{mM}$ $\mathrm{MgSO}_{4}, 0.1 \mathrm{mM} \mathrm{CaCl}_{2}, 0.5 \mu \mathrm{M} \mathrm{FeCl}_{3}, 28 \mathrm{mM}$ D-glucose or glycerol as sources of carbon. For the E. coli strain LB650 the M9 medium was supplemented with amino acids L-valine, L-isoleucine, Lmethionine, L-proline, L-serine (each $0.02 \%(w / v))$ and $3 \mu \mathrm{M}$ Thiamine. For the E. coli strain 
LB2003 the M9 medium was supplemented with $0.0066 \%(w / v)$ casein hydrolysate (acid) (Oxoid), $0.004 \%(w / v)$ L-proline and $3 \mu \mathrm{M}$ Thiamine. For experiments with defined potassium concentrations, the $\mathrm{KH}_{2} \mathrm{PO}_{4}$ salt was replaced by $\mathrm{NaH}_{2} \mathrm{PO}_{4}$ and $\mathrm{KCl}$ was added as indicated. If not specified different, IPTG was used at a concentration of $50 \mu \mathrm{M}$ and L-arabinose at $0.005 \%(w / v)$.

DNA manipulation - Transformation of E. coli was performed using standard procedures (Sambrook et al., 1989). Plasmid DNA was extracted using the NucleoSpin Plasmid Kit (Macherey and Nagel). Commercially available restriction enzymes, T4 DNA ligase and DNA polymerases were used as recommended by the manufacturers. DNA fragments were purified using the PCR purification kit (Qiagen). DNA sequences were determined by the dideoxy chain termination method (Microsynth, Göttingen, Germany). Chromosomal DNA of L. monocytogenes or B. subtilis was isolated using the NucleoSpin Microbial DNA Kit (Macherey and Nagel). Chromosomal DNA of S. aureus $\mathrm{COL}$ was a kindly provided by Dr. Jan Pané-Farré (University of Greifswald, Germany). Oligonucleotides were purchased from Sigma-Aldrich (Germany).

Tab. 3.1 Strains

\begin{tabular}{|c|c|c|c|}
\hline Name & Genotype & Description & Reference \\
\hline \multicolumn{4}{|l|}{ E. coli } \\
\hline LB2003 & $\begin{array}{l}\mathrm{F}^{-} \text {aroE rpsL metE thi gal rha kup1 (trkD1) } \\
\triangle k d p A B C 5 \triangle \operatorname{trk} A \operatorname{aroE}^{+}\end{array}$ & Potassium uptake studies & $\begin{array}{l}\text { Stumpe \& Bakker, } \\
1997\end{array}$ \\
\hline LB650 & $\begin{array}{l}\mathrm{F}^{-} \text {thi lacZ gal rha kup1 (trkD1) } \triangle k d p A B C 5 \\
\Delta t r k H\left(\mathrm{Cm}^{\mathrm{R}}\right) \Delta \operatorname{trkG}_{\left(\mathrm{Kan}^{\mathrm{R}}\right)}\end{array}$ & Potassium uptake studies & $\begin{array}{l}\text { Stumpe \& Bakker, } \\
1997\end{array}$ \\
\hline $\begin{array}{l}\text { NEB T7 Ex- } \\
\text { press } 19\end{array}$ & $\begin{array}{l}\text { MiniF lacla }\left(\mathrm{Cm}^{\mathrm{R}}\right) / \text { fhuA2 lacZ::T7 gene1 } \\
\text { [lon] ompT gal sulA11 R(mcr- } \\
\left.\text { 73::miniTn10--Tet }{ }^{\mathrm{S}}\right) 2[\mathrm{dcm}] \mathrm{R}(\mathrm{zg} b- \\
\left.\text { 210::Tn10--Tet }{ }^{\mathrm{S}}\right) \text { endA1 } \Delta(\mathrm{mcrC}- \\
\text { mrr)114::IS10 }\end{array}$ & $\begin{array}{l}\text { Protein expression and } \\
\text { DRaCALA }\end{array}$ & New England Biolabs \\
\hline Rosetta (DE3) & $\begin{array}{l}\mathrm{F}^{-} \text {ompT hsdS }\left(\mathrm{r}_{\mathrm{B}}^{-} \mathrm{m}_{\mathrm{B}^{-}}\right) \mathrm{gal} d \mathrm{dcm}(\mathrm{DE} 3) \\
\text { pRARE }\left(\mathrm{Cm}^{\mathrm{R}}\right)\end{array}$ & Protein expression & Novagen \\
\hline XL1-Blue & $\begin{array}{l}\text { recA1 endA1 gyrA96 thi-1 hsdR17 } \\
\text { supE44 relA1 lac [F' proAB lacla } Z \Delta M 15 \\
\left.\text { Tn10 }\left(\text { Tet }^{r}\right)\right]\end{array}$ & Cloning & Stratagene \\
\hline \multicolumn{4}{|l|}{ B. subtilis } \\
\hline 168 & $\operatorname{trpC2}$ & Wild type & Laboratory collection \\
\hline \multicolumn{4}{|c|}{ L. monocytogenes } \\
\hline EGD-e & Wild type & Serotype $1 / 2$ a strain & Laboratory collection \\
\hline
\end{tabular}

Plasmid construction - The genes encoding putative potassium transporters were introduced into the vector pWH844 allowing IPTG-dependent expression in E. coli (Schirmer et al., 1997). The $k_{i m A} A^{L m o}$ and $k i m A^{S a u}$ genes were amplified using the oligonucleotide pairs JH95/JH96 and $\mathrm{JH} 97 / \mathrm{JH} 98$, respectively (Tab. 3.2). The PCR products were digested with EcoRI and BamHI and ligated to $\mathrm{pWH} 844$ that was cut with the same enzymes. The resulting plasmids were designated as pBP384 and pBP385 (Tab. 3.3). To study the role of the C-terminal domain of KimA ${ }^{\text {Lmo }}$, we generated the plasmid pBP396. The truncated $k i m A^{L m o}$ gene was amplified using oligonucleotide pair $\mathrm{JH} 95 / \mathrm{JH} 120$, digested with EcoRI and BamHI and ligated to pWH844 cut with the same enzymes. The plasmid pBP371 for the expression of the L. monocytogenes ktrCD (Imo1023 and Imo0993) genes was constructed as follows. The $k \operatorname{tr} C$ and $k \operatorname{tr} D$ genes were amplified using the oligonucleotide pairs $\mathrm{JH} 59 / \mathrm{JH} 60$ and $\mathrm{JH} 61 / \mathrm{JH} 62$, respectively and fused by Splicing by Overhang Extension (SOE) PCR using primer pair JH59/JH62 (Horton et al., 1990). The resulting PCR product was digested with $\mathrm{EcORI}$ and $\mathrm{BamHI}$ and ligated to $\mathrm{pWH} 844$ cut with the same enzymes. The plasmids 
pBP370 and pBP373 were constructed for producing the wild type CdaA enzyme and the inactive D171N variant (Rosenberg et al., 2015). The $c d a A$ gene was amplified using the oligonucleotide pair JH51/JH52 and introduced into the Xbal/Pstl sites of pBAD33 (Guzman et al., 1995; Quintana et al., 2019). For the construction of plasmid pBP373, we used the oligonucleotide pair JH51/JH52 together with the 5'-phosphorylated oligonucleotide JR18 to introduce the D171N mutation via the combined chain reaction (Bi \& Stambrook, 1997; Quintana et al., 2019). The pBAD33 and pWH844 expression vectors have compatible selection markers and origin of replications allowing the co-expression of potassium transporter genes (from pWH844) and $c d a A$ variants (from pBAD33). The plasmids pBP345, pBP346 and pBP347 were constructed to study the binding of c-di-AMP to KtrC, the cytosolic domains of $\operatorname{KimA}^{\mathrm{Lmo}}$ (aa 452-607) and $\mathrm{KimA}^{\text {Sau }}$ (aa 452-609). The respective genes were amplified using the oligonucleotide pairs GH5/GH6, GH7/GH8 and GH9/GH10, digested with BamHI/Sall and ligated to pWH844 cut with the same enzymes. The genes encoding the full-length $\mathrm{KimA}^{\mathrm{Lmo}}$ and $\mathrm{Kim}^{\mathrm{Sau}}$ proteins as well as the $\mathrm{C}$-terminally truncated $\mathrm{KimA}^{\mathrm{Lmo}}$ variant (aa 1-455) were amplified using oligonucleotide pairs JH142/JH96, JH143/JH98 and JH142/JH120, respectively. The PCR products were digested with Sacl/BamHI and ligated to pGP172 (Merzbacher et al., 2004) cut with the same enzymes. The resulting plasmids were designated as pBP265 ( $\left.k i m A^{L m o}\right)$, pBP267 ( $k i m A^{\text {Sau }}$ ) and pBP266 ( $k i m A^{L m o} \Delta C$-terminus). The plasmids are suitable for the IPTG-dependent overproduction of the transporters with an N-terminal Strep-tag II in the E. coli strain Rosetta (DE3).

\section{Tab. 3.2 Oligonucleotides}

\begin{tabular}{|c|c|c|}
\hline Name & $\begin{array}{l}\text { Restriction sites are underlined, complementary regions are in bold, se- } \\
\text { quences } 5^{\prime} \rightarrow 3^{\prime}\end{array}$ & Purpose \\
\hline GH5 & AAAGGATCCATGAAAGAAGGATTTGCAGTCATCGGTCTTG & Fwd. $k \operatorname{tr} C^{\mathrm{Lmo}}($ BamHI) \\
\hline GH6 & TTTGTCGACTTATTGAATTTTTTTCTTGTAGTCGTTCAATGTCATCATCC & Rev. $k \operatorname{trC} C^{\mathrm{Lmo}}(\mathrm{Sall})$ \\
\hline $\mathrm{GH} 7$ & AAAGGATCCCATTACCGGAAAGTTGGACCACAACTTAG & $\begin{array}{l}\text { Fwd. } \operatorname{kim}^{\mathrm{Lmo}}(\text { aa } 452-\mathrm{x}) \\
\text { (BamHI) }\end{array}$ \\
\hline GH8 & TTTGTCGACTTATTCTTTTAAATGATAAGGATATGTGGAAACTACTACATCC & Rev. $\operatorname{kim}^{\mathrm{Lmo}}(\mathrm{Sall})$ \\
\hline GH9 & AAAGGATCCCATTATCGAGATATCGCAGAACAATTACGTTCTG & $\begin{array}{l}\left.\text { Fwd. } k i m A^{\text {Sau }} \text { (aa } 452-x\right) \\
\text { (BamHI) }\end{array}$ \\
\hline GH10 & $\begin{array}{l}\text { TTTGTCGACCTATTTTTTTAAGTTTAAATGGAATTGTACAT- } \\
\text { ACGTTAACATTCTTTTTG }\end{array}$ & Rev. $k i m A^{\text {Sau }}($ Sall) \\
\hline JH51 & AAATCTAGACACGGAGGTGAAGTGATGGATTTTTCCAATATGTCGATATTGCAT & $\begin{array}{l}\text { Fwd. } c d a A \text { (Xbal), Quintana } \\
\text { et al., } 2019\end{array}$ \\
\hline JH52 & TTTCTGCAGTCATTCGCTTTTGCCTCCTTTCCA & $\begin{array}{l}\text { Rev. cdaA (Pstl), Quintana } \\
\text { et al., } 2019\end{array}$ \\
\hline JH59 & AAAGAATTCAAGGAGGTAACGTACACATGAAAGAAGG & Fwd. $k \operatorname{trC} C^{\mathrm{Lmo}}(E c o \mathrm{RI})$ \\
\hline $\mathrm{JH} 60$ & АATCTTCTGCTAAGTACGGCTTTTTATTGAATTTTTTTCTTGTAGTCGTTCAATG & Rev. $k \operatorname{tr} C^{\mathrm{Lmo}}$ \\
\hline JH61 & CAATAAAAAGCCGTACTTAGCAGAAGATTAAAGCTTGTTTTGGCACG & Fwd. $k \operatorname{tr} D^{\mathrm{Lmo}}$ \\
\hline JH62 & TTTGGATCCTTAACCAGTAATAATTTTCTCTTTTGGTAAACGAATC & Rev. $k t r D^{\mathrm{Lmo}}($ BamHI) \\
\hline JH95 & AAAGAATTCAAAGGTAGGGAATACAATGGCTTCGCC & Fwd. $k_{i m} A^{\text {Lmo }}(E c o R I)$ \\
\hline JH96 & TTTGGATCCCTCTTGTTATTCTTTTAAAATGATAAGGATATGTGGAAAC & Rev. $\operatorname{kimA}^{\mathrm{Lmo}}($ BamHI) \\
\hline JH97 & AAAGAATTCAAAGGAATAGGAGATTATGTTCAATCAATTTAAAAGAC & Fwd. $k i m A^{\mathrm{Sau}}(E c o R I)$ \\
\hline $\mathrm{JH} 98$ & TTTGGATCCGAATCTATTTTTTAAGTTTAAATGGAATTGTACATACGTTAAC & Rev. kimA $A^{\text {Sau }}($ BamHI) \\
\hline $\mathrm{JH} 120$ & TTTGGATCCTTATTTCCGGTAATGATGTCTTGTACGATGGAAAAC & $\begin{array}{l}\text { Rev. } \text { kimA Amo }^{\text {Lmo }} \text { (a x-455) } \\
\text { (BamHI) }\end{array}$ \\
\hline $\mathrm{JH} 142$ & AAAGAGCTCGATGGCTTCGCCGCTAAAAAGACTATTAATCG & Fwd. $\operatorname{kim}^{\mathrm{Lmo}}(\mathrm{Sacl})$ \\
\hline $\mathrm{JH} 143$ & AAAGAGCTCGATGTTCAATCAATTTAAAAGACTTATTATAGGGCAACC & Fwd. $k i m A^{\text {Sau }}($ Sacl $)$ \\
\hline JR18 & P-GAATACACCGCTTCATAATGGAGCAGTTATTATTAA & $\begin{array}{l}\text { 5'-phosphorylated } \\
\text { GAT } \rightarrow \text { AAT (CdaA D171N), } \\
\text { Rosenberg et al., } 2015\end{array}$ \\
\hline
\end{tabular}


Drop dilution assay - Single colonies of the $E$. coli strain LB650 harboring the plasmids pWH844, pBP371, pBP372, pBP384, pBP385 or pBP396 were taken from LB-K plates and used to inoculate $4 \mathrm{ml}$ LB-K medium supplemented with kanamycin, ampicillin and chloramphenicol. The cultures were incubated at $37^{\circ} \mathrm{C}$ and $220 \mathrm{rpm}$. The pre-cultures were used to inoculate $4 \mathrm{ml} \mathrm{M9}$ medium supplemented with glucose, antibiotics and $50 \mathrm{mM} \mathrm{KCl}$ to an $\mathrm{OD}_{600}$ of 0.001 . The cultures were incubated for about $16 \mathrm{~h}$ at $37^{\circ} \mathrm{C}$. Next day, the cultures were used to inoculate $10 \mathrm{ml}$ of the same medium to an $\mathrm{OD}_{600}$ of 0.1 . At an $\mathrm{OD}_{600}$ between 0.3 and 0.5 the cells were harvested by centrifugation at $3300 \mathrm{~g}$ for $10 \mathrm{~min}$ at room temperature. The cell pellets were washed twice in $10 \mathrm{ml}$ of $\mathrm{M} 9$ medium lacking $\mathrm{KCl}$. The cell suspension was adjusted to an $\mathrm{OD}_{600}$ of 0.1 and $5 \mu \mathrm{l}$ of the diluted cells were spotted onto M9 minimal media plates, which were incubated for $24 \mathrm{~h}$ at $37^{\circ} \mathrm{C} . \mathrm{M} 9$ plates were prepared by mixing $2 \times \mathrm{M} 9$ medium (pre-warmed to $37^{\circ} \mathrm{C}$ ) and $2 \times$ Bacto agar (prewarmed to $70^{\circ} \mathrm{C}$ before mixing). The finial medium contained glucose as a carbon source, $10 \mathrm{mM}$ $\mathrm{KCl}$, and $50 \mu \mathrm{M}$ IPTG if required.

Tab. 3.3 Plasmids

\begin{tabular}{|c|c|c|}
\hline Name & Insert/Features & Reference \\
\hline pBAD33 & $\mathrm{P}_{\mathrm{BAD}}$ cat araC & Guzman et al., 1995 \\
\hline pWH844 & $\mathrm{P}_{\mathrm{T} 5} b l a$ & Schirmer et al., 1997 \\
\hline pGP172 & $\mathrm{P}_{\mathrm{T} 7}$ bla & Merzbacher et al., 2004 \\
\hline pBP265 & pGP172-Strep-tag II-kimA Lmo & This work \\
\hline pBP266 & pGP172-Strep-tag II- kimA $A^{\text {Lmo }} \Delta \mathrm{C}$-terminus & This work \\
\hline pBP267 & pGP172-Strep-tag II-kimA $A^{\text {sau }}$ & This work \\
\hline pBP345 & pWH844-His $6-k t r C^{L m o}$ & This work \\
\hline pBP346 & pWH844-His 6 - kimA ${ }^{\text {Sau }}$ C-terminal domain & This work \\
\hline pBP347 & pWH844-His6-kimA ${ }^{\text {Sau }}$ C-terminal domain & This work \\
\hline pBP370 & pBAD33-cdaA & Quintana et al., 2019 \\
\hline pBP371 & pWH844-ktrC Lmo-ktrD Lmo & This work \\
\hline pBP372 & pWH844-ktrAB & Gundlach et al., 2017 \\
\hline pBP373 & pBAD33-cdaA (D171N) & Quintana et al., 2019 \\
\hline pBP384 & pWH844-kimA $A^{L m o}$ & This work \\
\hline pBP385 & pWH844-kimA & This work \\
\hline pBP396 & pWH844-kimA $A^{\text {Lmo }}(\Delta \mathrm{C}$-terminus) & This work \\
\hline
\end{tabular}

Determination of kinetic parameters of the potassium transporters - To determine the growth characteristics of the $E$. coli strain LB650 synthesizing potassium transporters from L. monocytogenes and $S$. aureus, the bacteria were grown until the early exponential phase, harvested by centrifugation at $3300 \mathrm{~g}$ for $10 \mathrm{~min}$. The pellet was resuspended in $10 \mathrm{ml} \mathrm{M9}$ medium with glucose, ampicillin, $50 \mu \mathrm{M}$ IPTG and without $\mathrm{KCl}$. The cells were incubated for $1 \mathrm{~h}$ at $37^{\circ} \mathrm{C}$, harvested by centrifugation and washed twice. The cultures were adjusted to an $\mathrm{OD}_{600}$ of 0.2 and $50 \mu \mathrm{l}$ were used to inoculate a 96-well plate (Microtest Plate $96-W e l l, F$, Sarstedt) containing $50 \mu l$ of M9 medium with glucose, ampicillin, $50 \mu \mathrm{M}$ IPTG and $\mathrm{KCl}$ concentrations ranging from 0 to $100 \mathrm{mM}$. The 96-well plate was incubated at $37^{\circ} \mathrm{C}$ with medium orbital shaking at $237 \mathrm{cpm}(4 \mathrm{~mm})$ in an Epoch 2 Microplate Spectrophotometer, equipped with the Gen5 software (02.09.2001; BioTek Instruments) and growth $\left(\mathrm{OD}_{600}\right)$ measured in 15 min intervals. The growth rates were calculated $(\mu=$ $\left.\left(2.303 \cdot\left(\log \left(\mathrm{OD}_{2}\right)-\log \left(\mathrm{OD}_{1}\right)\right)\right) /\left(\mathrm{t}_{2}-\mathrm{t}_{1}\right)\right)$, plotted against the $\mathrm{KCl}$ concentrations and fitted to the Michaelis-Menten equation using the solver tool of Excel 2013 (Microsoft), to calculate $V_{\max }\left(\mu\left[h^{-}\right.\right.$ $\left.{ }^{1}\right]$ ) and the apparent $\mathrm{K}_{\mathrm{M}}[\mathrm{mM} \mathrm{KCl}]$. 
c-di-AMP in vivo inhibition assay - The potassium transporter deficient E. coli strain LB2003 was co-transformed with the plasmid pWH844 or derivatives (pBP371, pBP384, or pBP396) and the pBAD33 derivatives (pBP370 or pBP373) on LB-K plates containing $0.5 \%(w / v)$ glucose, ampicillin and chloramphenicol. Single colonies were used to inoculate $4 \mathrm{ml}$ LB-K medium containing $0.2 \%$ $(w / v)$ glucose, ampicillin and chloramphenicol and the exponentially growing cultures were used to inoculate M9 medium containing $0.2 \%(w / v)$ glycerol and $0.02 \%(w / v)$ glucose to an $\mathrm{OD}_{600}$ of 0.001 . The cultures were incubated overnight at $37^{\circ} \mathrm{C}$ and used to re-inoculate the same medium (without glucose) to an $\mathrm{OD}_{600}$ of 0.1 . After reaching early exponential phase $\left(\mathrm{OD}_{600} 0.3-0.5\right)$, the cells were washed and $100 \mu \mathrm{l}$ of the suspensions were used to inoculate a 96-well plate. The M9 medium was supplemented with glycerol, $50 \mu \mathrm{M}$ IPTG, ampicillin, chloramphenicol, $\mathrm{KCl}$ and with or without $\mathrm{L}$-arabinose. Final concentrations of $\mathrm{KCl}$ were equal to the determined $\mathrm{K}_{\mathrm{M}}$ values (see Tab. 3.4) and no or $0.005 \%(w / v)$ L-arabinose was present, as indicated. Growth was monitored in an Epoch 2 Microplate Spectrophotometer, equipped with the Gen5 software (02.09.2001; BioTek Instruments), as described above.

Protein expression and DRaCALA - The binding of c-di-AMP to the potassium transporters was analyzed using the $E$. coli strain Rosetta (DE3). Single colonies were used to inoculate $10 \mathrm{ml}$ LB-K medium containing carbenicillin and chloramphenicol. After incubation overnight at $30^{\circ} \mathrm{C}$, the precultures were used to inoculate $1.5 \mathrm{ml}$ of LB-K medium to an $\mathrm{OD}_{600}$ of $0.1 .1 \mathrm{mM}$ IPTG was added at an $\mathrm{OD}_{600}$ of $1.0-1.5$ to induce gene expression. After incubation for $4 \mathrm{~h}$, the cultures were harvested by centrifugation $\left(3300 \mathrm{~g}, 10 \mathrm{~min}, 4^{\circ} \mathrm{C}\right.$ ), the cell pellets were resuspended in $150 \mu \mathrm{l}$ Tris$\mathrm{NaCl}$ buffer (10 mM Tris, pH 8.0, $100 \mathrm{mM} \mathrm{NaCl}$ ). Cells are lysed by three freeze-thaw cycles of $-80^{\circ} \mathrm{C}$ and room temperature. The Differential radial capillary action of ligand assay (DRaCALA) was performed by mixing $1 \mu \mathrm{L}$ of ${ }^{32} \mathrm{P}$-c-di-AMP with $20 \mu \mathrm{L}$ of cell lysates. After an incubation for one minute, $2 \mu \mathrm{L}$ of the mixture was spotted on dry nitrocellulose, dried, exposed to phosphorimager screen and imaged using FLA-7000 phosphorimager. The fraction bound was calculated using the inner and total areas and intensities as described previously (Roelofs et al., 2011).

Microscopic analysis - Derivatives of the LB650 strain harboring the plasmids pWH844 (empty plasmid), pBP372, or pBP384 were cultivated in 4 ml LB-K medium containing ampicillin, kanamycin and chloramphenicol at $37^{\circ} \mathrm{C}$. Next day, the cultures were washed twice and used to inoculate $10 \mathrm{ml} \mathrm{M9}$ medium (containing $22.05 \mathrm{mM} \mathrm{KH}_{2} \mathrm{PO}_{4}$ ) with or without $1 \mathrm{mM}$ IPTG to an $\mathrm{OD}_{600}$ of 0.1 . Cells were transferred to standard microscope slides (Carl Roth) and examined using an Axioskop $40 \mathrm{FL}$ fluorescence microscope, equipped with an Axio-Cam MRm digital camera, objectives of the Neofluar series at 1,000-fold magnification and the AxioVision Rel 4.8.2 software (Carl Zeiss). Images were later on equally processed using the ImageJ 1.48 software (Schneider et al., 2012).

\section{Results}

In silico identification of potassium transporters from L. monocytogenes - Both, B. subtilis and S. aureus contain well-described potassium uptake systems. B. subtilis uses the high-affinity transporters KtrAB and KimA and the low-affinity transporter KtrCD (Gundlach et al.,2017; Holtman et al.,2003). By contrast, S. aureus relies on the high-affinity transporter KdpFABC, whose synthesis and activity is controlled by the two-component system KdpDE and c-di-AMP, respectively (Gründling, 2003; Moscoso et al.,2015; Price-Whelan et al.,2013). S. aureus also contains the low-affinity potassium transport systems KtrCB and KtrCD, sharing the accessory protein KtrC (Price-Whelan et al.,2013). A BLASTp sequence analysis revealed that the L. monocytogenes genome codes for the KdpABCDE (Lmo2682-Lmo2678) and KtrCD (Lmo1023, Lmo0993) proteins, which show about 
$31-56 \%$ and $51-64 \%$ overall amino acid identity, respectively, with the homologs from S. aureus and $B$. subtilis. The $k d p F$ gene does not exist in the genome of $L$. monocytogenes. A homolog of the high-affinity potassium transporter KimA from B. subtilis is also present in L. monocytogenes and S. aureus (Gundlach et al.,2017). The KimA homologs from B. subtilis, L. monocytogenes and S. aureus are from now on designated as KimA ${ }^{\mathrm{Bsu}}$, KimA ${ }^{\mathrm{Lmo}}\left(\mathrm{Lmo}^{2130}\right)$ and $\mathrm{KimA}^{\mathrm{Sau}}$ (Sacol2443), respectively. KimA $A^{\mathrm{Lmo}}$ and $\mathrm{Kim}^{\mathrm{Sau}}$ show about $59 \%$ and $57 \%$ overall amino acid identity, respectively, with the $B$. subtilis homolog (Fig. 3.1, A). The membrane topology was illustrated using the web-based tool Protter (Gründling, 2013). Like KimA ${ }^{\text {Bsu }}$ also KimA ${ }^{\text {Lmo }}$ contains an N-terminal extracellular domain, 11 transmembrane (TM) helices and a C-terminal intracellular domain, which might be important for activity control of the transporter (Fig. 3.1, B). To conclude, although B. subtilis, L. monocytogenes and S. aureus are phylogenetically related, each species uses a different set of transporters for potassium uptake.

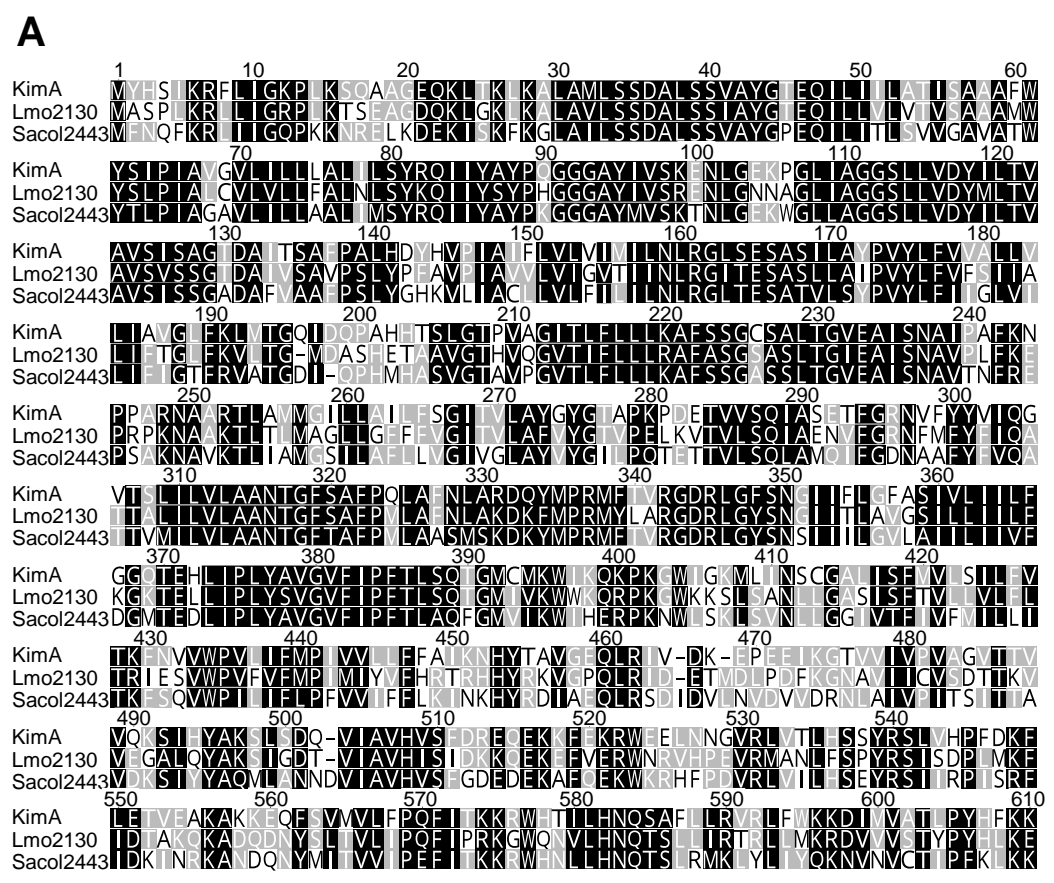

B

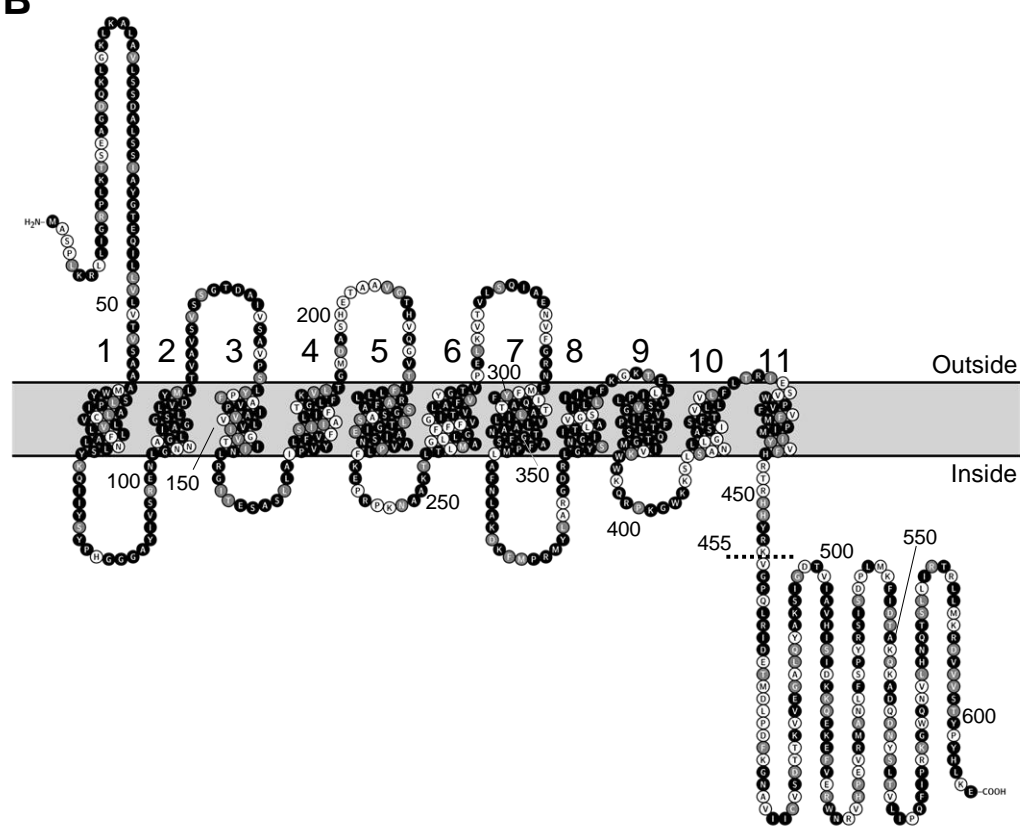

Fig. 3.1 Alignment of KimA homologs and domain organization of the $\operatorname{Kim}^{\mathrm{Lmo}}$ protein. $A$, MUSCLE alignment of the KimA ${ }^{\text {Bsu }}$, KimA ${ }^{\text {Lmo }}$ and $\operatorname{KimA}^{\mathrm{Sau}}$ homologs from B. subtilis, L. monocytogenes (Lmo2130) and S. aureus (Sacol2443), respectively, generated with Geneious (46). Amino acids in black, grey and white have an amino acid similarity of over $80 \%, 60-80 \%$, or less than $60 \%$, respectively $B$, predicted membrane topology of $\mathrm{KimA}^{\mathrm{Lmo}}$ overlaid with a MUSCLE alignment between $\operatorname{KimA}^{\text {Bsu }}$ and $\operatorname{KimA}^{\text {Lmo }}$. The dashed line indicates the position at which the KimA ${ }^{\text {Lmo }}$ protein was truncated. Amino acids in black are identical; amino acids in grey are similar and amino acids in white are non-similar. 
In vivo activities of KimA and KtrCD potassium transporters - To assess whether KimA $A^{\operatorname{Lmo}}$ and $K \operatorname{trCD}$ from L. monocytogenes are active in potassium transport, we cloned the $k i m A$ and $k \operatorname{trCD}$ genes using the plasmid pWH844, which allows IPTG-dependent expression of heterologous genes in E. coli (Schirmer et al.,1997). We also cloned a truncated kimA $^{\text {Lmo }}$ gene encoding the $\Delta C$-KimA ${ }^{\text {Lmo }}$ variant lacking 152 amino acids of the $\mathrm{C}$-terminal cytosolic domain. Furthermore, we cloned the $\mathrm{kimA}^{\mathrm{Sau}}$ gene from S. aureus, to evaluate whether KimA homologs from other Firmicutes are involved in potassium uptake. The resulting plasmids were used to transform the $E$. coli strain LB650 that is unable to take up potassium via the native uptake systems Kup, KdpABC, TrkG and TrkH (Stumpe and Bakker, 1997). The strain is suitable to study potassium transporters because it is only viable in minimal medium supplemented with potassium concentrations above $15 \mathrm{mM} \mathrm{KCl}$ (see Fig. 3.4). The empty plasmid and a plasmid encoding the $B$. subtilis ktrAB genes, which were previously shown to mediate potassium transport in E. coli LB650 (Gundlach et al., 2017), served as negative and positive controls, respectively. The cells were grown during the day in M9 medium with $50 \mathrm{mM} \mathrm{KCl}$ and without IPTG induction, collected by centrifugation, washed in potassiumfree medium and propagated on M9 minimal medium plates without and with $10 \mathrm{mM}$ IPTG. As shown in Fig. 3.2, with the exception of the strain harboring the plasmid for $k i m A^{\text {Sau }}$ expression, the bacteria could not grow in the absence of IPTG. The weak growth of the cells containing the $k i m A^{\text {Sau }}$ gene could be due to a leaky promoter, the high affinity of the encoded KimA ${ }^{\text {Sau }}$ transporter for potassium and perhaps a stronger translation due to using the natural ribosomal binding sites of the different transporter (see below). By contrast, the strains carrying the $k \operatorname{tr} A B^{B s u}$, $\operatorname{kim} A^{L m o}, k \operatorname{trCD}{ }^{L m o}$, could grow with low amounts of $\mathrm{K}^{+}$when these genes were induced with IPTG (Fig. 3.2).

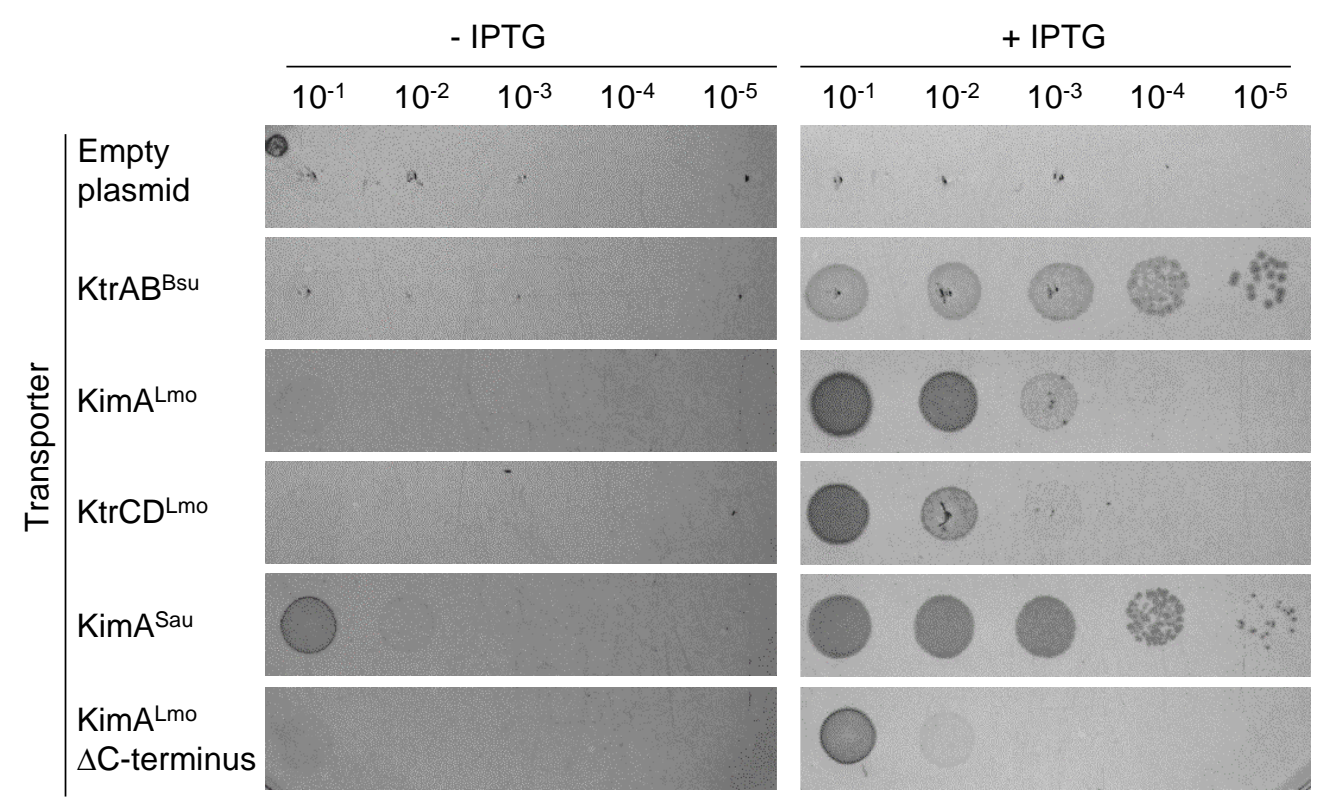

Fig. 3.2 Drop dilution assay to assess the activities of putative potassium transporters. E. coli $\mathrm{LB} 650$ strains harboring plasmids pWH844 (empty plasmid), pBP372 (ktrAB ${ }^{\text {Bsu }}$, positive control), pBP384 (kimA ${ }^{\text {Lmo }}$ ), pBP396 (kimA $A^{\text {Lmo }} \Delta C$-terminus), pBP385 ( Kim $^{\text {Sau }}$ ) and pBP371 ( $\left.k \operatorname{trCD}{ }^{L m o}\right)$ were grown to an $\mathrm{OD}_{600}$ of 0.3-0.5 in M9 minimal medium supplemented with $50 \mathrm{mM} \mathrm{KCl}$. The cells were washed for $1 \mathrm{~h}$ in potassium-free $\mathrm{M} 9$ medium, the $\mathrm{OD}_{600}$ was adjusted to 0.1 , serial tenfold diluted and $5 \mu$ of the diluted cell suspensions were plated on $\mathrm{M} 9$ plates containing $10 \mathrm{mM} \mathrm{KCl}$. IPTG was added to a final concentration of $50 \mu \mathrm{M}$ to induce the expression of the transporter genes. The plates were incubated for $24 \mathrm{~h}$ at $37^{\circ} \mathrm{C}$. 
Moreover, the $k i m A^{L m o}$ variant lacking the C-terminal domain ( $k i m A^{L m o} \Delta C$ ) also restored growth, albeit less well than the strain producing the full-length protein. These results indicate that the $\mathrm{N}$ terminal extracellular domain and the $11 \mathrm{TM}$ helices of KimA ${ }^{\mathrm{Lmo}}$ from L. monocytogenes are sufficient for mediating potassium import in $E$. coli (Fig. 3.2). Expression of $k \operatorname{trA} B^{B s u}$ from B. subtilis restores growth on low potassium concentrations agreeing with previous reports that $\operatorname{Ktr}^{A B}{ }^{B s u}$ is a high-affinity potassium transporter (Gundlach et al., 2017; Holtman, et al., 2003). Expression of $\mathrm{KimA}^{\mathrm{Sau}}$ from S. aureus E. coli strain LB650 resulted in a much better growth than those strains expressing Kim $A^{\mathrm{Lmo}}$ and $\mathrm{KtrCD}$ indicating that KimA ${ }^{\text {Sau }}$ is likely a high-affinity potassium transporter (Fig. 3.2). Thus, KimA ${ }^{\mathrm{Lmo}}$ and $\mathrm{KtrCD}{ }^{\mathrm{Lmo}}$ from L. monocytogenes as well as KimA ${ }^{\text {Sau }}$ from S. aureus are indeed potassium transporters.

Apparent affinities of KimA and KtrCD for potassium - To determine the affinities of KimA ${ }^{\operatorname{Lmo}}$, the $\operatorname{KimA}^{\mathrm{Lmo}} \Delta \mathrm{C}$-terminus variant $\left(\Delta \mathrm{C}\right.$-KimA $\left.{ }^{\mathrm{Lmo}}\right)$ and $\mathrm{KtrCD}$ from L. monocytogenes and KimA ${ }^{\mathrm{Sau}}$ from $S$. aureus, we determined the growth rates of the $E$. coli strain LB650 synthesizing the potassium transporters in $\mathrm{M} 9$ minimal medium supplemented with different amounts of potassium. The strains carrying the empty plasmid and expressing the $B$. subtilis $k t r A B$ genes served as negative and positive controls, respectively. The growth rates were plotted against the potassium concentrations and fitted to the Michaelis-Menten equation (Gundlach et al., 2017). The $V_{\max }$ values and the apparent affinities are summarized in Tab. 3.4. As shown in Fig. 3.3 and 3.4, each E. coli strain required a different concentration of external potassium to reach half-maximal growth: The strains synthesizing the transporters $\mathrm{KtrCD}^{\mathrm{Lmo}}, \mathrm{KimA}^{\mathrm{Lmo}}, \Delta \mathrm{C}-\mathrm{Kim}^{\mathrm{Lmo}}, \mathrm{Kim}^{\mathrm{Sau}}$ and $\mathrm{KtrAB}^{\mathrm{Bsu}}$ required $6.31 \pm 1.8 \mu \mathrm{M}, 0.35 \pm 0.1 \mu \mathrm{M}, 2.99 \pm 0.65 \mu \mathrm{M}, 0.14 \pm 0.02 \mu \mathrm{M}$ and $0.03 \pm 0.01 \mu \mathrm{M}$, respectively.

Tab. 3.4 Michaelis-Menten constants of the potassium transporters

\begin{tabular}{|c|c|c|c|c|c|c|}
\hline & Empty Vector & KtrAB Bsu & $\operatorname{Kim} A^{\text {Lmo }}$ & $\begin{array}{c}\text { KimA }^{\text {Lmo }} \\
\Delta \text { C-terminus }\end{array}$ & KtrCD Lmo & $\operatorname{Kim}^{\mathrm{Sat}}$ \\
\hline $\begin{array}{c}\text { Apparent } \mathbf{K}_{\mathbf{M}} \\
{[\mathrm{mM} \mathrm{KCl}]}\end{array}$ & $\begin{array}{c}\mathbf{5 8 . 2 8} \\
\pm 14.60\end{array}$ & $\begin{array}{c}0.03 \\
\pm 0.01\end{array}$ & $\begin{array}{c}\mathbf{0 . 3 5} \\
\pm 0.10\end{array}$ & $\begin{array}{c}\mathbf{2 . 9 9} \\
\pm 0.65\end{array}$ & $\begin{array}{c}\mathbf{6 . 3 1} \\
\pm 1.80\end{array}$ & $\begin{array}{c}\mathbf{0 . 1 4} \\
\pm 0.02\end{array}$ \\
\hline$V_{\max }\left[\mu\left(h^{-1}\right)\right]$ & $\begin{array}{c}0.82 \\
\pm 0.13\end{array}$ & $\begin{array}{c}0.73 \\
\pm 0.04\end{array}$ & $\begin{array}{c}0.52 \\
\pm 0.04\end{array}$ & $\begin{array}{c}0.40 \\
\pm 0.01\end{array}$ & $\begin{array}{c}0.59 \\
\pm 0.10\end{array}$ & $\begin{array}{c}\mathbf{0 . 6 2} \\
\pm 0.05\end{array}$ \\
\hline
\end{tabular}

These results demonstrate that $\mathrm{KtrCD}^{\mathrm{Lmo}}$ and $\mathrm{KimA}^{\mathrm{Lmo}}$ from L. monocytogenes are transporters with low and moderately high affinities for potassium, respectively. Moreover, the C-terminal intracellular domain of KimA ${ }^{\mathrm{Lmo}}$ is important for full activity of the transporter (Fig. 3.1, B; Fig. 3.4). In contrast to $\mathrm{KtrCD}^{\mathrm{Lmo}}$ and $\mathrm{Kim}^{\mathrm{Lmo}}$, $\mathrm{Kim}^{\mathrm{Sau}}$ from $S$. aureus is a high-affinity potassium transporter, which is in line with the observation that the $E$. coli strain LB650 synthesizing KimA ${ }^{\mathrm{Sau}}$ and KtrAB ${ }^{\text {Bsu }}$ grew comparatively well with low amounts of potassium (Fig. 3.2). 

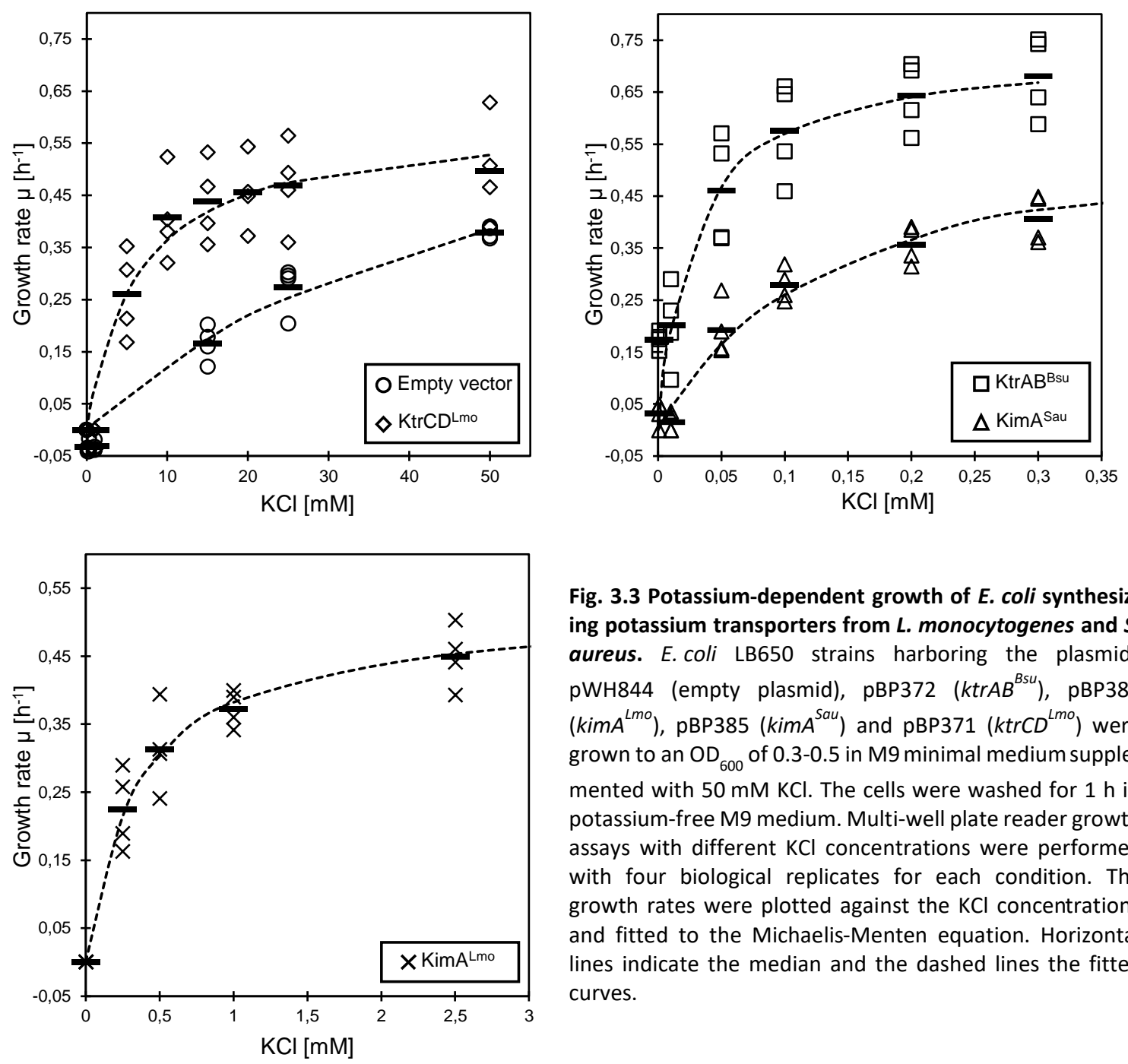

Fig. 3.3 Potassium-dependent growth of $E$. coli synthesizing potassium transporters from $L$. monocytogenes and $S$. aureus. E. coli LB650 strains harboring the plasmids pWH844 (empty plasmid), pBP372 (ktrAB $\left.{ }^{B s u}\right)$, pBP384 $\left(k i m A^{\text {Lmo }}\right)$, pBP385 $\left(k i m A^{\text {Sau }}\right)$ and pBP371 $\left(k \operatorname{trCD} D^{\text {Lmo }}\right)$ were grown to an $\mathrm{OD}_{600}$ of 0.3-0.5 in $\mathrm{M9}$ minimal medium supplemented with $50 \mathrm{mM} \mathrm{KCl}$. The cells were washed for $1 \mathrm{~h}$ in potassium-free $\mathrm{M} 9$ medium. Multi-well plate reader growth assays with different $\mathrm{KCl}$ concentrations were performed with four biological replicates for each condition. The growth rates were plotted against the $\mathrm{KCl}$ concentrations and fitted to the Michaelis-Menten equation. Horizontal lines indicate the median and the dashed lines the fitted curves.

Inhibition of KimA and KtrCD potassium transport activity by c-di-AMP - Several recent studies indicate that c-di-AMP is essential for viability of Gram-positive bacteria such as B. subtilis, L. lactis, L. monocytogenes, S. agalactiae and $S$. aureus because the nucleotide controls the influx of osmolytes like potassium, of which the accumulation leads to cell lysis due to water uptake (Devaux et al., 2018; Gundlach et al., 2017; Pham et al., 2018; Woodward et al., 2010; Zeden et al., 2018). Thus, either synthesis of the potassium transporters their activity or both need to be tightly regulated. As shown in Fig. 3.5, the IPTG-dependent overexpression of the $k \operatorname{trAB} B^{B s u}$ and $\operatorname{kim} A^{\text {Lmo }}$ genes encoding high-affinity potassium transporters $\mathrm{KtrAB}^{\mathrm{Bsu}}$ and $\mathrm{Kim} \mathrm{A}^{\mathrm{Lmo}}$, respectively, in E. coli during growth in $\mathrm{M} 9$ minimal medium caused a strong increase of the cellular volume. Moreover, the growth of $E$. coli synthesizing the higher affinity $\mathrm{KtrAB}^{\mathrm{Bs} u}$ transporter was in addition significantly reduced as illustrated by the decline of the optical density (Fig. 3.5; upper right corners). By contrast, in the absence of the inducer IPTG the growth and the volume of the cells containing the $k \operatorname{tr} A B^{B s u}$ and $k i m A^{L m o}$ genes were indistinguishable from that of the cells carrying the empty vector. Thus, once sufficient potassium has been taken up by the bacteria to cope with the osmolarity of the environment, the activities of osmolyte transporters have to be reduced to prevent further ion uptake and cell lysis. In case of KtrAB from $S$. aureus it has indeed been demonstrated that the activity of the transporter is inhibited by c-di-AMP (Corrigan et al., 2013; Bai et al., 2014). Like $\mathrm{KimA}^{\mathrm{Bsu}}$ from B. subtilis the KimA ${ }^{\mathrm{Lmo}}$ homolog from L. monocytogenes belongs to a novel class of high-affinity potassium transporters (see above; Gundlach et al., 2017). However, whether 
c-di-AMP directly binds to $\mathrm{Kim}^{\mathrm{Lmo}}$ and $\mathrm{KtrCD}^{\mathrm{Lmo}}$ to inhibit the transport activity of the proteins is unknown.

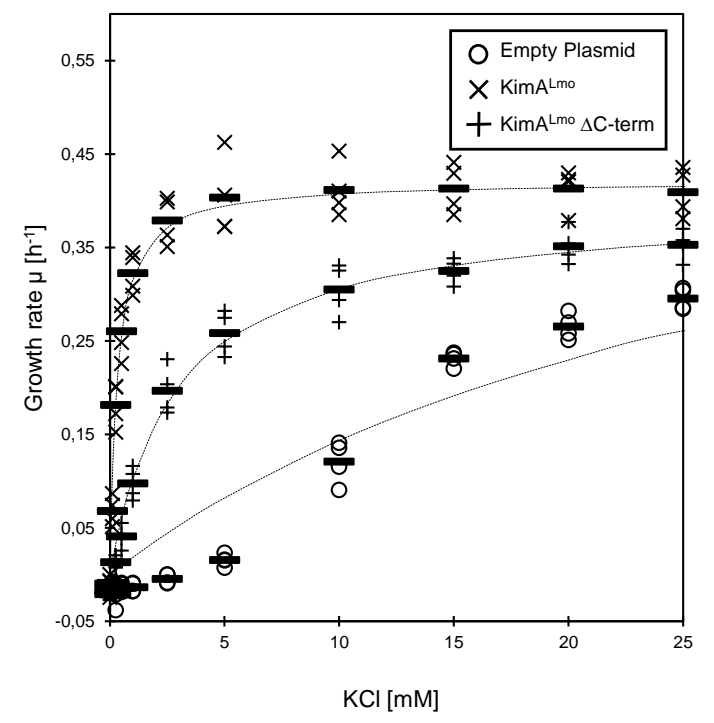

Fig. 3.4 Potassium-dependent growth of $E$. coli synthesizing the full-length and the C-terminally truncated $\operatorname{KimA}^{\text {Lmo }}$ protein. E. coli LB650 strains harboring the plasmids pWH844 (empty plasmid), pBP384 ( $\left(k i m A^{L m o}\right)$ and pBP396 ( $k$ imA ${ }^{\text {Lmo }} \Delta$ C-terminus) were grown to an $\mathrm{OD}_{600}$ of 0.3-0.5 in M9 minimal medium supplemented with $50 \mathrm{mM}$ $\mathrm{KCl}$. The cells were washed for $1 \mathrm{~h}$ in potassium-free $\mathrm{M9}$ medium. Multi-well plate reader growth assays with different $\mathrm{KCl}$ concentrations were performed with four biological replicates for each condition. The growth rates were plotted against the $\mathrm{KCl}$ concentrations and fitted to the Michaelis-Menten equation. Horizontal lines indicate the median and the dashed lines the fitted curves.

To assess whether c-di-AMP affects the activity of KimA ${ }^{\mathrm{Lmo}}$ and $\mathrm{KtrCD}{ }^{\mathrm{Lmo}}$, we established a co-expression system using the $E$. coli strain LB2003, which carries unmarked mutations in the $k d p$, kup and trk genes and enable the use of multiple plasmids encoding chloramphenicol and ampicillin resistance genes (Stumpe \& Bakker, 1997). Like the E. coli strain LB650, LB2003 is deficient in the $\mathrm{Kdp}$, Kup and Trk potassium uptake systems, and is therefore only able to grow at low potassium concentrations when synthesizing a potassium transporter. Moreover, E. coli lacks c-di-AMP-producing and c-di-AMP-degrading enzymes, which is a prerequisite to assess the phenotypic effect of c-di-AMP on the activity of KimA ${ }^{\mathrm{Lmo}}$ and $\mathrm{KtrCD}^{\mathrm{Lmo}}$. The plasmids pBP384 $\left(\mathrm{kimA}^{\mathrm{Lmo}}\right)$, pBP396 $(\Delta C$ $\left.k i m A^{L m o}\right)$ and pBP371 $\left(k \operatorname{tr} C D^{L m o}\right)$ were used for the IPTG-dependent expression of $\triangle C$-KimA ${ }^{\text {Lmo }}$, $\mathrm{KimA}^{\mathrm{Lmo}}$ and $\mathrm{KtrCD}{ }^{\mathrm{Lmo}}$, respectively. The empty plasmid pWH844 served as a negative control. The L. monocytogenes DAC CdaA and the inactive CdaA* variant D171N (Rosenberg et al., 2015) are encoded by the arabinose-inducible plasmids pBP370 and pBP373, respectively (Quintana et al., 2019). The strains carrying pWH844, pBP384, pBP396 and pBP371 as well as either of the two DAC encoding plasmids were grown in $\mathrm{M} 9$ minimal medium supplemented with $30 \mathrm{mM}, 0.35 \mathrm{mM}$, $3 \mathrm{mM}$ and $7 \mathrm{mM} \mathrm{KCl}$, respectively, conditions that allow half-maximal growth of the bacteria. As shown in Fig. 3.6, growth of the strains carrying the empty plasmid pWH844, and synthesizing the active and the catalytically inactive $\mathrm{CdaA}$ and $\mathrm{CdaA}^{*}$ variants, respectively, was not reduced. Thus, neither the DAC proteins nor c-di-AMP affect growth of the $E$. coli strain. By contrast, growth of the bacteria synthesizing $\mathrm{KimA}^{\mathrm{Lmo}}$ and $\mathrm{KtrCD}{ }^{\mathrm{Lmo}}$ was reduced when the active DAC CdaA was coproduced, indicating that c-di-AMP inhibits the transporter with a moderately high affinity for potassium and to a lesser extent also the low-affinity transporter (Fig. 3.6). In case of KtrCD ${ }^{\mathrm{Lmo}}$, a slight growth delay was also observed in the absence of an inducer for expression of wild type CdaA. Growth was not affected in the absence of a functional DAC and thus of c-di-AMP production. Moreover, c-di-AMP did not affect the activity of the C-terminally truncated $\Delta C$-KimA ${ }^{\text {Lmo }}$ variant, indicating that the $\mathrm{C}$-terminal intracellular domain of the transporter contributes to c-di-AMP-dependent regulation (see Discussion). Surprisingly, c-di-AMP did not inhibit the activity of KimA ${ }^{\text {Sau }}$ from S. aureus (data not shown). To conclude, the potassium transporters KimA ${ }^{\text {Lmo }}$ and KtrCD ${ }^{\mathrm{Lmo}}$ from L. monocytogenes are both inhibited by c-di-AMP. 


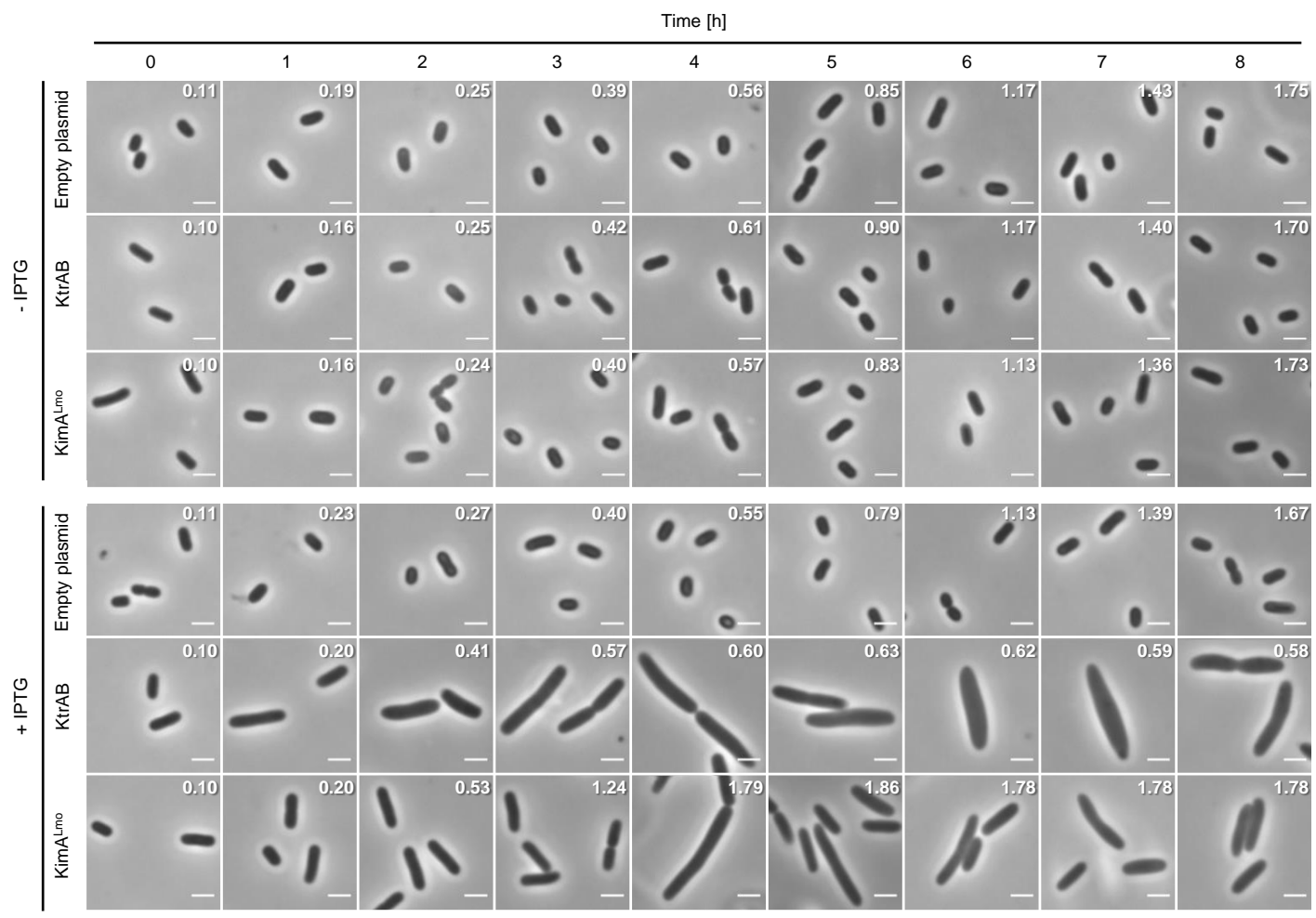

Fig. 3.5 Impact of unregulated potassium import on the cell volume of $E$. coli. Derivatives of the $E$. coli strain LB650 harboring plasmids pWH844 (empty plasmid), pBP372 $\left(k \operatorname{trAB}{ }^{B s u}\right)$ and pBP384 $\left(k i m A^{L m o}\right)$ were grown overnight in LB-K medium. The cells were washed and cultivated in M9 medium without and with $1 \mathrm{mM} \mathrm{IPTG}$ for the induction of the transporter genes. The $\mathrm{OD}_{600}$, which is shown in the right upper corner of the microscopic pictures, was measured in hourly intervals. Scale bar, $2 \mu \mathrm{m}$.

c-di-AMP interaction with the KimA homologs and $K \operatorname{tr} C D^{L m o}$ - To assess the interaction between c-di-AMP and the potassium transporters KimA ${ }^{\text {Lmo }}, \Delta C-K_{i m A}{ }^{\text {Lmo }}$, KimA $A^{\text {Sau }}$, and $K \operatorname{trCD}{ }^{\text {Lmo }}$ we performed a DRaCALA (see Experimental procedures). We also tested the interaction between c-di-AMP and the 156 and 158 amino acid long cytosolic C-terminal domains of KimA ${ }^{\text {Lmo }}$ and $\mathrm{Ki}$ $\mathrm{mA}^{\mathrm{Sau}}$, respectively. This domain could be involved in the c-di-AMP-dependent control of KimA potassium transport activity. The lysate of the E. coli strain DH5 $\alpha$ containing the empty plasmids pWH844 or pGP172 served as negative controls. While the majority of the proteins showed no specific interaction with c-di-AMP in the DRaCALA assay, the potassium transporter KtrCD ${ }^{\mathrm{Lmo}}$ gave a positive result (Fig. 3.7). To conclude, the potassium transport activity of $\mathrm{KtrCD}{ }^{\mathrm{Lmo}}$ from L. monocytogenes is inhibited by c-di-AMP in vivo and the nucleotide binds to the KtrC subunit of the $\mathrm{K} \operatorname{trCD}^{\mathrm{Lmo}}$ transporter in vitro. 


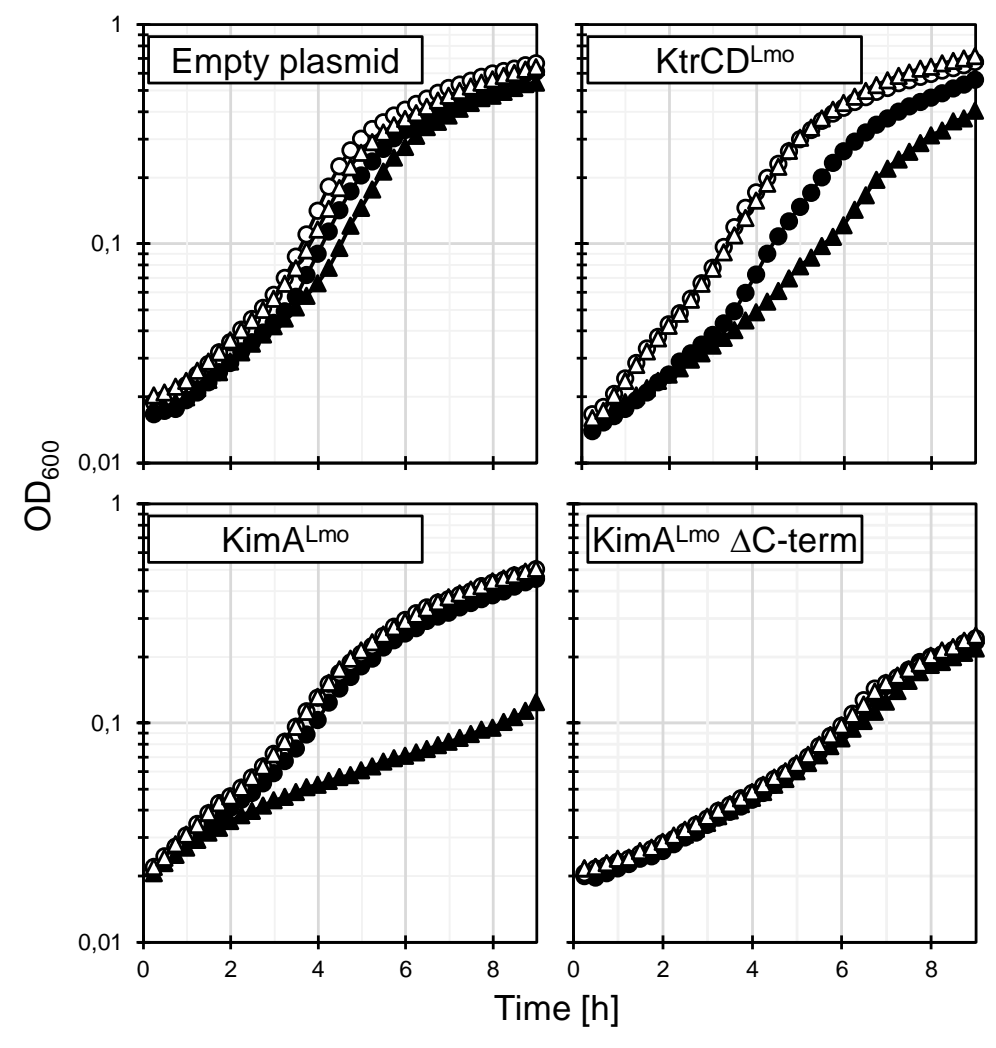

Fig. 3.6 Inhibition of potassium transporters by c-di-AMP. The E. coli strain LB2003 harboring the plasmids pWH844 (empty plasmid), pBP371 $\left(k \operatorname{trCD} D^{\mathrm{Lmo}}\right), \quad$ pBP384 $\left(\right.$ kim $\left.A^{\text {Lmo }}\right)$, pBP396 $\left(k i m A^{\text {Lmo }} \Delta \mathrm{C}\right.$-terminus) and either pBP370 (cdaA; filled symbols) or pBP373 (cdaA D171N; unfilled symbols) were grown to an $\mathrm{OD}_{600}$ of $0.3-0.5$ in $\mathrm{M9}$ medium and washed for one hour in potassium-free M9 medium. The growth assays were performed with (triangles) or without (circles) $0.005 \%(w / v)$ L-arabinose and at $\mathrm{KCl}$ concentrations that are equal to the $\mathrm{K}_{\mathrm{M}}$ values of the transporters. Shown are the mean values of three biological replicates.

\section{Discussion}

In the present study, we have identified and characterized the potassium transporters KtrCD ${ }^{\operatorname{Lmo}}$ and KimA ${ }^{\text {Lmo }}$ from $L$. monocytogenes. We have also demonstrated that $S$. aureus possesses a homolog of KimA (Sacol2443). The KimA homologs from S. aureus and L. monocytogenes belong to a novel class of high-affinity potassium transporters that are active at low external potassium concentrations (Gundlach et al., 2017). By contrast, the KtrCD ${ }^{L m o}$ transporter from L. monocytogenes has a much lower affinity for potassium than the homolog from B. subtilis (Holtmann et al., 2003). As the external concentrations of potassium are rather low, it is tempting to speculate that $L$. monocytogenes possesses an additional high-affinity potassium transporter to be able to compete with other bacteria when the extracellular potassium is scarce. The phylogenetically related bacteria $B$. subtilis and $S$. aureus contain two high-affinity potassium transport systems that are active during growth at low potassium concentrations. $B$. subtilis employs the high-affinity potassium transporters KtrAB and KimA under potassium limiting growth conditions (Gundlach et al., 2017; Holtmann et al., 2003). S. aureus relies on the high-affinity transporter KdpFABC, whose synthesis and activity is regulated by the two-component system KdpDE (Gründling, 2013; Moscoso et al., 2016; Price-Whelan et al., 2013). Moreover, KimA ${ }^{\text {Sau }}$ could be important for growth of $S$. aureus when the extracellular potassium concentrations are low. In L. monocytogenes, additional potassium transport may be mediated by the KdpABCDE (Lmo2678-Lmo2682) system, as it is the case in $S$. aureus. The precise role of the KdpABCDE system and the KimA ${ }^{\text {sau }}$ transporter from L. monocytogenes and $S$. aureus, respectively, in potassium homeostasis and their regulation in these bacteria remains to be investigated. Interestingly, also the $k \operatorname{tr} C D$ genes of $L$. monocytogenes might be subject to regulation on the level of gene expression. $k \operatorname{tr} C$ is located as last gene in an operon with two genes of unknown function and the genes liaFSR. The later three encode a three-component system involved in cell envelope stress response and a deletion of the lias gene leads to higher expression of the operon, including $k \operatorname{trC}$ (Fritsch et al., 2011). 
A

B

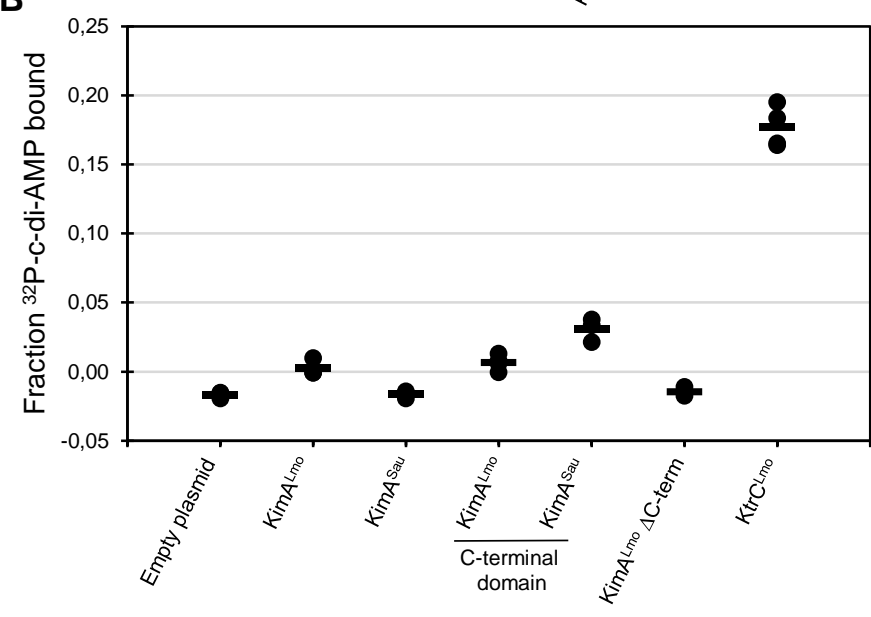

Fig. 3.7 Interaction between c-di-AMP and potassium transporters determined by DRaCALA. A, Autoradiographs showing the interaction between radiolabeled c-di-AMP $\left({ }^{32} \mathrm{P}\right.$-c-di-AMP) and the potassium transporters as well as the truncated variants that are present in whole cell lysates of the E. coli strain T7 Express 19 carrying the plasmids pGP172 (empty plasmid), or the derivatives pBP265 (kimA $\left.{ }^{\text {Lmo }}\right)$, pBP266 (kimA ${ }^{\text {Lmo }}$ $\Delta$ C-terminus), pBP267 (kimA $\left.{ }^{\text {Sau }}\right), \quad$ or pWH844 (empty plasmid), or the derivatives pBP346 (kimA ${ }^{\text {Lmo }}$ C-terminal domain), pBP347 (kimA ${ }^{\text {Sau }}$ C-terminal domain) and pBP345 (ktrC $\left.C^{\perp m o}\right)$. Both empty vectors showed similar non-binding (data not shown). $B$, Fraction bound of ${ }^{32} \mathrm{P}$-c-di-AMP is shown for lysates from $E$. coli induced overnight for the expression of the indicated gene. Data of three biological replicates and their mean values are depicted.

The $k t r D$ gene is also the last gene of an operon, encoding two putative manganese transporters with a $y y b P-y k o Y R N A$ motif in the promoter region of the operon, indicating potential regulation by this RNA motif. It will be interesting to elucidate the regulatory mechanisms of expression and activity of these transporters in L. monocytogenes.

As stated above, during growth under hyperosmotic conditions many bacteria take up potassium ions to prevent water efflux from the cytosol and to increase the cellular turgor (Kempf \& Bremer, 1998). Once the cellular turgor has been adjusted to the environmental osmolarity, the transport of potassium ions across-the cell membrane has to be reduced to prevent osmotic swelling and cell lysis (Wood, 1999; Wood, 2011). A reduction of the ion uptake might be either achieved by proteolytic degradation or by controlling the activity of the transporters through binding of lowmolecular weight ligands. It has indeed been shown that transport systems are rapidly degraded when the respective substrates are not available (Horak \& Wolf, 1997). However, the cellular turgor is a physical variable that changes rapidly and needs to be tightly adjusted (Wood, 1999; Wood, 2011). Therefore, it is obvious that the regulation by inhibition is favorable over degradation, so the bacteria can quickly re-adjust if conditions change without having to depend on de novo synthesis of the transporter. Hence, the tight control of the cellular turgor requires the existence of low-molecular weight ligands, which specifically modulate the activity of potassium transporters and other osmolyte uptake systems. Indeed, in S. aureus it has been shown that the low-affinity potassium transporters KtrCB and KtrCD are inhibited by the second messenger c-di-AMP that binds to the RCK_C (regulator of conductance of $\mathrm{K}^{+}$) domain of the KtrC gating component (Corrigan et al., 2013). It also has recently been shown that B. subtilis KtrA and KtrC are functional redundant for potassium transporter regulation, if expressed in $E$. coli and that $\mathrm{KtrC}$ has a higher affinity for c-di-AMP, as KtrA (Rocha et al., 2019). Moreover, c-di-AMP binds to the CabP protein and prevents potassium uptake by the CabP-TrkH protein complex in S. pneumoniae (Bai et al., 2014). The cytoplasmatic regulatory subunit KtrC of the KtrCD potassium transporter is also bound by c-di-AMP in Mycoplasma pneumoniae (Blötz et al., 2017). Differential regulation would require a kind of local signaling as it has been demonstrated for the second messenger c-di-GMP, which could be achieved by co-localization of synthesis and degradation machineries with the 
targets of c-di-AMP signaling (Sarenko et al., 2017). Here, we have demonstrated for the first time that the potassium transporters $\operatorname{KtrCD}^{\mathrm{Lmo}}$ and $\mathrm{Kim}^{\mathrm{Lmo}}$ from $L$. monocytogenes are inhibited by the second messenger c-di-AMP. This study also shows that the uncontrolled influx of potassium ions via the KtrAB ${ }^{\text {Bsu }}$ and KimA ${ }^{\text {Lmo }}$ results in osmotic swelling of E. coli (Fig. 3.5). In an accompanying paper it has been shown that c-di-AMP inhibits the potassium transport activity of the KimA homolog from B. subtilis (Gundlach et al., 2019). Thus, c-di-AMP is required to reduce potassium uptake to toxic levels. Interestingly, c-di-AMP also controls the uptake of potassium at the level of transcription. For instance, c-di-AMP inhibits the sensor kinase KdpD of the KdpDE two-component system and thus reduces the expression of the $k d p F A B C$ operon encoding the high-affinity KdpFABC potassium transport system from S. aureus (Moscoso et al., 2016; Price-Whelan et al., 2013). Moreover, binding of c-di-AMP to the, in L. monocytogenes absent, ydaO riboswitch in the untranslated region of the ktrAB and kimA mRNAs prevents transcription beyond the riboswitch in $B$. subtilis, thereby reducing expression of the high-affinity potassium transporters KtrAB and KimA, respectively (Gundlach et al., 2017; Nelson et al., 2013). It should be noted that c-di-AMP also inhibits the uptake of other osmolytes such as glycine betaine and carnitine (Devaux et al., 2018; Huynh et al., 2016; Pham et al., 2018; Schuster et al., 2016; Whiteley et al., 2017). Thus, c-di-AMP plays a central role in controlling the activities of potassium transporters and other osmolyte uptake systems, and the c-di-AMP-dependent regulation can occur at two different levels in a variety of bacteria. In the future, it will be interesting to investigate whether the synthesis of the $L$. monocytogenes potassium transporters $\mathrm{KimA}^{\mathrm{Lmo}}$ and $\mathrm{KtrCD}{ }^{\mathrm{Lmo}}$, which are both inhibited by c-di-AMP, is also regulated by the nucleotide. Moreover, it might be worth to study the role of the C-terminal cytosolic domain in the activity control of the KimA potassium transporter. Unfortunately, we could not find an interaction between c-di-AMP and the C-terminal domain of KimA ${ }^{\text {Lmo }}$ in E. coli cell lysates. However, this domain seems to be important for the c-di-AMP-mediated regulation of KimA ${ }^{\mathrm{Lmo}}$ since the C-terminally truncated variant lacking 156 amino acids did not respond to the nucleotide in vivo.

Recently, it has been demonstrated that the control of potassium uptake is an essential function of c-di-AMP in B. subtilis (Gundlach et al., 2017). A B. subtilis strain lacking all c-di-AMP-producing enzymes was only viable in medium containing low potassium concentrations. c-di-AMP is also essential in bacteria like L. monocytogenes, S. agalactiae and S. aureus to prevent the uptake of osmolytes to toxic levels (Devaux et al., 2018; Pham et al., 2018; Whiteley et al., 2015). However, in these bacteria the control of glycine betaine and amino acid uptake seems to be the essential function of c-di-AMP. Thus, phylogenetically related bacteria have evolved species-specific mechanisms to regulate the cellular turgor, but they all use c-di-AMP in this essential process (Commichau et al., 2018). It remains to be elucidated how c-di-AMP controls potassium homeostasis in L. monocytogenes. Moreover, it will be crucial to identify the osmo-signal sensing mechanism of the c-di-AMP system, which could be conserved among different bacteria (Commichau et al., 2018; Pham et al., 2018).

\section{Acknowledgements}

We thank Jasmin Gömann for the help with some experiments. We are grateful to Sabine Lentes for technical support. This work was supported by the grant CO 1139/2-1 from the Deutsche Forschungsgemeinschaft via the Prioritiy Program SPP1879, the Fonds der Chemischen Industrie and the Max-Buchner-Forschungsstiftung (MBFSt-Kennziffer 3381) to FMC. 


\title{
4. Global effects of c-di-AMP on gene expression and protein biosynthesis in Listeria monocytogenes
}

Johannes Gibhardt, Samuel Hauf, Alexander Reder, Andrea Thürmer, Uwe Völker, Sven Halbedel and Fabian M. Commichau

Author contribution:

$J G$ performed the experiments and data evaluation, SH and AT and SH performed the RNA-Seq, AR and UV performed the protein quantifications, JG and FMC wrote the manuscript.

\begin{abstract}
The nucleotide second messenger cyclic di-AMP (c-di-AMP) has been associated with various crucial cellular functions. c-di-AMP is essential in Firmicutes under most growth conditions and has been extensively investigated in the model bacterium Bacillus subtilis and in pathogenic bacteria, like Listeria monocytogenes, Staphylococcus aureus or Streptococcus pneumoniae. It is synthesized by diadenylate cyclases (DACs) and degraded by specific phosphodiesterases (PDEs) and has been shown to regulate activity of proteins, regulate translation by binding to RNA structures and to control gene expression by regulation of transcriptional regulators. Thus, c-di-AMP can act on all levels of protein synthesis. The second messenger has been associated with regulation of osmotic homeostasis by regulation of potassium and compatible solute import, cell wall biosynthesis, DNA repair mechanisms, regulation of central carbon and nitrogen metabolism and to modulate other nucleotide second messenger signaling pathways, like the stringent response. To elucidate the impact of c-di-AMP on the overall cellular function, we investigated changes in transcriptome and proteome of a cdaA deletion mutant, encoding the sole DAC in L. monocytogenes under defined growth conditions in Listeria synthetic medium (LSM). Changes in gene expression and protein biosynthesis revealed a total of 95 differently regulated genes and 21 proteins with different abundance. The deregulated influences important processes as the central carbon metabolism, nitrogen metabolism, cell wall and lipid biosynthesis, ion transport or expression of motility associated genes. The present report will help to identify novel targets of the c-di-AMP regulatory network and deepen the understanding about c-di-AMP signaling.
\end{abstract}




\section{Introduction}

Bacteria have to adapt to various stimuli and compete with other organisms to thrive in their environment. It is therefore important for cells to have a repertoire of sensing and signal modulation machineries to integrate these stimuli and respond properly. One key feature is the modulation of gene expression and protein biosynthesis to react to immediate changes in conditions or the long-term adaptation by acquisition of genome amplifications and evolution of mutations (Andersson \& Hughes, 2009; López-Maury et al., 2008). The fastest response, despite modulating gene expression or protein biosynthesis, is the modulation of protein activity by direct influence of stimuli or through ligand binding. One example for a direct influence is the regulation of mechanosensitive channels that directly react on changes in turgor upon a hypoosmotic shock, before other cellular processes catch up with the changes in external osmolarity (Hoffmann, et al., 2008). An important tool that has evolved for the integration of environmental clues are second messenger molecules. Among them, nucleotide second messenger molecules play important role in adaptation to manifold environmental stimuli (Newton et al., 2016). c-di-AMP is one of those nucleotide second messenger molecules (Commichau et al., 2015; Corrigan et al., 2013). It is synthesized and degraded by different set ups of DACs and PDEs, respectively, in different bacterial species (Commichau et al., 2019). c-di-AMP has been connected to several important cellular functions, ranging from DNA damage repair, to cell wall metabolism, regulation of central carbon and nitrogen metabolism or lifestyle changes in different bacteria, like B. subtilis, L. monocytogenes, S. aureus, Lactococcus lactis or Mycobacterium smegmatis (Corrigan et al., 2011; Kaplan Zeevi et al., 2013; Luo \& Helmann, 2012; Mehne et al., 2013; Oppenheimer-Shaanan et al., 2011; Whiteley et al., 2013; Witte et al., 2008; Witte et al., 2013; Zhang \& He, 2013; Zhu et al., 2016). In the recent years however, it has been shown that the main function of c-di-AMP and the reason for its essentiality and toxicity, under different growth conditions (Gundlach et al., 2015), is the regulation of osmohomeostasis (Devaux et al., 2018; Gundlach et al., 2017; Huynh et al., 2016; Pham et al., 2018; Schuster et al., 2016; Whiteley et al., 2015; Whiteley et al., 2017; Zeden et al., 2018). It was therefore also proposed that many phenotypes of mutants that affect c-di-AMP signaling, especially the cell wall-associated ones that are seen in an altered resistance to $\beta$-lactam antibiotics, might be at least in part indirect effects due to the role of c-di-AMP as major regulator of osmotic homeostasis in firmicutes (Commichau et al., 2018). A link between c-di-AMP and cell wall metabolism however, seems to be established due to the regulation of CdaA activity, the sole DAC in many pathogenic firmicutes, by the phosphoglucosamine mutase GImM that is involved in peptidoglycan precursor synthesis and able to inhibit CdaA activity (Tosi et al., 2019; Zhu et al., 2016). c-di-AMP has furthermore been shown to be involved in cross-regulation with other nucleotide second messenger signaling pathways, such as the stringent response (Corrigan et al., 2015; Huynh et al., 2015; Rao et al., 2010; Whiteley et al., 2015). Due to this cross-talk c-di-AMP probably also influences other, still unknown pathway, like regulation of the pleiotropic transcriptional regulator CodY and thereby processes like the import of oligopeptides via the Opp transporter that are important for L. monocytogenes as osmolytes (Geiger \& Wolz, 2014; Whiteley et al., 2017). To understand these indirect and direct effects of c-di-AMP is crucial for understanding the regulation of those important cellular processes. It has for example been shown that a $B$. subtilis gdpP deletion mutant, which accumulates higher intracellular concentrations of c-di-AMP, leads to an inhibition of biofilm formation and facilitates expression of motility associated genes (Gundlach et al., 2016). Analysis of whole cell changes in gene expression and protein biosynthesis can therefore be a powerful tool to find novel direct and indirect processes that are regulated by a second messenger, such as c-di-AMP. We therefore investigated the impact of a $c d a A$ deletion that encodes the only c-di-AMP synthesizing enzyme in L. monocytogenes under defined conditions. To the best 
of our knowledge, this study is the first, to investigate the impact of lack of c-di-AMP on global changes in gene expression and protein biosynthesis. The transcriptomic and proteomic analyses revealed a broad impact of c-di-AMP on cellular functions and highlights possible new genes and whole operons that are directly or indirectly regulated by c-di-AMP, hence deepen our understanding of c-di-AMP signaling.

\section{Experimental procedures}

Bacterial strains and growth conditions - L. monocytogenes EGD-e and its derivatives were cultivated in $\mathrm{BHI}$ medium (Sigma-Aldrich) at $37^{\circ} \mathrm{C}$ and $220 \mathrm{rpm}$ if not specified otherwise. Escherichia coli was grown in LB medium at $37^{\circ} \mathrm{C}$ and $220 \mathrm{rpm}$ (strains see Tab.4.1). For agar plates, medium was supplemented with $15 \mathrm{~g} / \mathrm{l}$ Bacto Agar (Difco). Antibiotics and medium supplements were used with the following concentrations, if indicated: erythromycin $(5 \mu \mathrm{g} / \mathrm{ml})$, ampicillin $(100 \mu \mathrm{g} / \mathrm{ml}), \mathrm{X}$ Gal ( $100 \mu \mathrm{g} / \mathrm{ml}$; 5-bromo-4-chloro-3-indolyl- $\beta$-D-galactopyranoside; Sigma-Aldrich). As a minimal medium for L. monocytogenes the LSM medium (Whiteley et al., 2017) was used with previously described changes (see chapter 2). For pouring minimal medium agar plates, $2 \mathrm{X}$ concentrated medium was pre-warmed to $37^{\circ} \mathrm{C}$ and mixed with to $70^{\circ} \mathrm{C}$ pre-warmed $2 \times$ Bacto agar, directly before pouring the plates.

Tab. 4.1 Strains

\begin{tabular}{|c|c|c|c|}
\hline Name & Genotype & Description/Construction & Reference \\
\hline \multicolumn{4}{|l|}{ E. coli } \\
\hline XL1-Blue & $\begin{array}{l}\text { recA1 endA1 gyrA96 thi-1 hsdR17 supE44 } \\
\text { relA1 lac }\left[\mathrm{F}^{\prime} \text { proAB lac/a } Z \Delta \mathrm{M} 15 \mathrm{Tn} 10\left(\text { Tet }^{R}\right)\right]\end{array}$ & Cloning & Stratagene \\
\hline \multicolumn{4}{|c|}{ L. monocytogenes } \\
\hline EGD-e & Wild type & Serotype $1 / 2$ a strain & Laboratory collection \\
\hline BPL10 & $\Delta f l i l:: \mathrm{P}_{\text {alf }}-\mathrm{RBS}_{\mathrm{hly}}-\mathrm{mgfp}(\mathrm{A} 206 \mathrm{~K})$ & pBP351 $\rightarrow \rightarrow$ EGD-e & This work \\
\hline BPL23 & $\Delta p g p H$ & pBP355 $\rightarrow \rightarrow$ EGD-e & This work \\
\hline BPL24 & $\Delta p d e A$ & pBP356 $\rightarrow \rightarrow$ EGD-e & This work \\
\hline BPL77 & $\Delta c d a A$ & $\begin{array}{l}\text { Chromosomal deletion of } \\
\text { cdaA }\end{array}$ & Chapter 2 \\
\hline LMJR45 & $\Delta c d a R$ & $\begin{array}{l}\text { Chromosomal deletion of } \\
\text { cdaR }\end{array}$ & Rismondo et al., 2016 \\
\hline
\end{tabular}

DNA manipulation - DNA amplification via PCR and transformation of $E$. coli was performed using standard procedures (Sambrook et al., 1989). DNA fragments were purified using the PCR purification kit (Qiagen) and plasmid DNA was extracted using the NucleoSpin Plasmid Kit (Macherey and Nagel). Commercially available restriction enzymes, T4 DNA ligase and DNA polymerases were used as recommended by the manufacturers. DNA sequences were determined by the dideoxy chain termination method (Microsynth, Göttingen, Germany). Chromosomal DNA of L. monocytogenes was isolated using the NucleoSpin Microbial DNA Kit (Macherey and Nagel). Oligonucleotides were purchased from Sigma-Aldrich (Germany).

Plasmid construction - For the chromosomal replacement of the flil gene by mgfp (A206K) and the chromosomal deletions of the $p g p H$ and pdeA genes, plasmids pBP351, pBP355 and pBP356 were constructed, respectively (plasmids are listed in Tab. 4.2). The $m g f p$ gene was amplified from plasmid pBP43 using oligonucleotides JHO1 and JHO2 (for the oligonucleotides see Tab. 4.3), with JHO1 
attaching the artificial alf4 promoter and the ribosomal binding site of the L. monocytogenes gene hly to facilitate $m g f p$ expression (Cascante-Estepa et al., 2016; Stannek, 2015). The resulting PCR product was digested with EcoRI and ligated to pSH185 (pMAD- $\Delta$ flil; Halbedel et al., 2012), which was digested with the same enzymes and via sequencing a plasmid identified, in which the gfp gene lies in reverse orientation to the operon to minimize effects of the promoter of the operon on the $g f p$ gene and of the alf4 promoter on the following genes of the operon. For construction of pBP355 and pBP356, the up- and downstream regions of the genes $p g p H$ and pdeA were amplified using oligonucleotide pairs $\mathrm{JH} 17 / \mathrm{JH} 18$ and $\mathrm{JH} 19 / \mathrm{JH} 20$ for $p g p H$ and $\mathrm{JH} 13 / \mathrm{JH} 14$ and $\mathrm{JH} 15 / \mathrm{JH} 16$ for $p d e A$, respectively. The resulting PCR products were fused by SOE PCR using oligonucleotide pairs $\mathrm{JH} 17 / \mathrm{JH} 20$ and $\mathrm{JH} 13 / \mathrm{JH} 16$ for $p g p H$ and $p d e A$, respectively (Horton et al., 1990). The resulting up- and downstream fusion PCR products were subsequently digested with EcoRI and BamHI and ligated to pMAD that was digested using the same enzymes (Arnaud et al., 2004).

Tab. 4.2 Plasmids

\begin{tabular}{l|l|l}
\hline Name & Insert/Features & Reference \\
\hline pMAD & bla ermC bgaB & Arnaud et al., 2004 \\
pSH185 & pMAD- $\Delta$ flil & Halbedel et al., 2012 \\
pBP43 & pUS19-mgfp (A206K) & Cascante-Estepa et al., 2016 \\
pBP351 & pMAD- $\Delta f l i l:: P_{\text {alf4-RBS }}$ - $m g f p($ A206K) & This work \\
pBP355 & pMAD- $\Delta p g p H$ & This work \\
pBP356 & pMAD- $\Delta p d e A$ & This work
\end{tabular}

L. monocytogenes strain construction - BPL10 was constructed by the chromosomal replacement of flil by mgfp to have a non-motile and fluorescent strain. To have strains with defects in c-di-AMP degradation and investigate those effects, strains BPL23 and BPL24 were constructed, harboring chromosomal deletions of pgpH or pdeA, respectively. BPL10, BPL23 and BPL24 were constructed as follows. Preparing electrocompetent cells and the electroporation is described elsewhere (Monk et al., 2008; chapter 2). The EGD-e wt was electroporated with plasmids pBP351, pBP355 or pBP356, respectively, and plated on BHI medium with erythromycin and X-Gal at $30^{\circ} \mathrm{C}$ for up to $72 \mathrm{~h}$. Single, blue colonies were streaked on the same medium and incubated for up to $72 \mathrm{~h}$ at $42^{\circ} \mathrm{C}$ to force integration of the plasmids into the different gene loci. Several blue colonies were used to inoculate $5 \mathrm{ml}$ of $\mathrm{BHI}$ medium without antibiotics at $30^{\circ} \mathrm{C}$ for $4 \mathrm{~h}$, temperature was shifted to $42^{\circ} \mathrm{C}$ for $6 \mathrm{~h}$, after which serial dilutions were plated on BHI agar plates with X-Gal and incubated at $42^{\circ} \mathrm{C}$ for up to $72 \mathrm{~h}$. Erythromycin-sensitive, X-Gal negative bacteria were subjected to colony PCR as described elsewhere (Dussurget et al., 2002). flil replacement and pgpH or pdeA deletion was confirmed by Sanger sequencing.

Tab. 4.3 Oligonucleotides

\begin{tabular}{|c|c|c|}
\hline Name & $\begin{array}{l}\text { Restriction sites are underlined, complementary regions are in bold, se- } \\
\text { quences } \mathbf{5}^{\prime} \rightarrow \mathbf{3}^{\prime}\end{array}$ & Purpose and reference \\
\hline JHO1 & $\begin{array}{l}\text { AAAGAATTCTAATTCTTGTCAAGTGAAGGCGCGCTATGCTACAATACAGCTT- } \\
\text { GGAAATCTA- } \\
\text { GAAGGAGAGTGAAACCCATGAGTAAAGGAGAAGAACTTTTCACTGGAG }\end{array}$ & $\begin{array}{l}\text { Fwd. } \quad \mathrm{P}_{\text {alf4 }}-\mathrm{RBS} S_{h l y}-m g f p \\
\text { (A206K) (EcoRI) }\end{array}$ \\
\hline $\mathrm{JH} 02$ & TTTGAATTCTTATTTGTATAGTTCATCCATGCCATGTGTAATC & $\begin{array}{l}\text { Rev. mgfp (A206K) } \\
\text { (EcoRI) }\end{array}$ \\
\hline $\mathrm{JH} 13$ & AAAGAATTCGAATGCCTACACATCAAGGTATGGG & $\begin{array}{l}\text { Fwd. pdeA upstream re- } \\
\text { gion (EcoRI) }\end{array}$ \\
\hline
\end{tabular}




\begin{tabular}{|c|c|c|}
\hline Name & $\begin{array}{l}\text { Restriction sites are underlined, complementary regions are in bold, se- } \\
\text { quences } 5^{\prime} \rightarrow 3^{\prime}\end{array}$ & Purpose and reference \\
\hline $\mathrm{JH} 14$ & TACGCATCAATGTCGACCCCATTTTTTTTACATACCTTTTGCCTG & $\begin{array}{l}\text { Rev. pdeA upstream re- } \\
\text { gion (Sall) }\end{array}$ \\
\hline $\mathrm{JH} 15$ & AAAAATGGGGTCGACATTGATGCGTATTGGAAGGGAGAAAC & $\begin{array}{l}\text { Fwd. } p d e A \text { downstream } \\
\text { region (Sall) }\end{array}$ \\
\hline JH16 & TTTGGATCCGCGCCTCATAAACCGTTAATACATCG & $\begin{array}{l}\text { Rev. pdeA downstream } \\
\text { region }(B a m \mathrm{HI})\end{array}$ \\
\hline JH17 & AAAGAATTCCTGTACAGCATACGACTGCCGT & $\begin{array}{l}\text { Fwd. pgpH upstream re- } \\
\text { gion (EcoRI) }\end{array}$ \\
\hline $\mathrm{JH} 18$ & СTCCTATTTCAGTCGACTCTCTAATTTTGTTTCCCTTCGTAAGCT & $\begin{array}{l}\text { Rev. pgpH upstream re- } \\
\text { gion (Sall) }\end{array}$ \\
\hline JH19 & CAAAATTAGAGAGTCGACTGAAATAGGAGGGCAAAAGATGACG & $\begin{array}{l}\text { Fwd. pgpH downstream } \\
\text { region (Sall) }\end{array}$ \\
\hline $\mathrm{JH} 2 \mathrm{O}$ & TTTGGATCCTCGCTGTGTTCACCATCTCTAGC & $\begin{array}{l}\text { Rev. pgpH downstream } \\
\text { region (BamHI) }\end{array}$ \\
\hline
\end{tabular}

Fwd. $=$ forward, Rev. $=$ reverse

Motility assay - The L. monocytogenes wild type strain, the flil mutant BPL10, the $p g p H$ and pdeA mutants BPL23 and BPL24, respectively, and the cdaA mutant BPL77 (see chapter 2) were used to assess the impact of c-di-AMP on the motility. Strains were cultivated in $5 \mathrm{ml} \mathrm{LSM}$ overnight at $37^{\circ} \mathrm{C}$ and $220 \mathrm{rpm}$. The pre-cultures were used to inoculate $5 \mathrm{ml}$ of LSM medium to an $\mathrm{OD}_{600}$ of 0.1 and the cells were incubated at $37^{\circ} \mathrm{C}$ and $220 \mathrm{rpm}$. Cells were harvested and resuspended in fresh $L S M$ medium after reaching an $O_{600}$ of about 0.5 and $1 \mu \mathrm{I}$ of $\mathrm{OD}_{600} 1.0$ cell suspensions were used to stab inoculate $\mathrm{LSM}$ or $\mathrm{BHI}$ soft agar plates $\left(0.3 \%(\mathrm{w} / \mathrm{v})\right.$ agar). Plates were incubated at $25^{\circ} \mathrm{C}$ or $37^{\circ} \mathrm{C}$, as indicated and imaged every $24 \mathrm{~h}$.

Growth of L. monocytogenes wt and $\Delta \mathrm{cdaA}$ mutant for transcriptomic and proteomic analysis - To analyze the changes in the transcriptome and proteome of $L$. monocytogenes, if the sole c-di-AMP synthesizing enzyme CdaA is not present, the wild type strain EGD-e and the $c d a A$ clean deletion mutant BPL77 (see chapter 2) were cultivated and processed as follows. Single colonies were propagated from LSM agar plates to $10 \mathrm{ml} \mathrm{LSM}$ overnight at $37^{\circ} \mathrm{C}$ and $220 \mathrm{rpm}$. Pre-cultures were used to inoculate $100 \mathrm{ml} \mathrm{LSM}$ medium to an $\mathrm{OD}_{600}$ of 0.05 and incubated at $37^{\circ} \mathrm{C}$ and $220 \mathrm{rpm}$. For the analysis of the proteome $40 \mathrm{OD}_{600}$ units of cells were harvested by rapid cooling in liquid nitrogen to slow down cellular processes and subsequent centrifugation after reaching an $\mathrm{OD}_{600}$ of about 0.5 at $3300 \mathrm{~g}$ and $4^{\circ} \mathrm{C}$ for $10 \mathrm{~min}$. Cell pellets were washed twice in ZAP ( $50 \mathrm{mM}$ Tris pH 7.5 and $200 \mathrm{mM} \mathrm{NaCl}$ ), with centrifugation steps in between. Resulting pellets were frozen in liquid nitrogen and stored at $-80^{\circ} \mathrm{C}$ until further processing. Growth for the analysis of the transcriptome was performed similar. When the cultures reached $\mathrm{OD}_{600}$ of $0.5 \pm 0.05,25 \mathrm{ml}$ were collected and quenched by adding of $25 \mathrm{ml}$ ice cold killing buffer $(20 \mathrm{mM} \mathrm{Tris-HCl} \mathrm{pH} \mathrm{7.5,5} \mathrm{mM} \mathrm{MgCl}, 20 \mathrm{mM}$ $\mathrm{NaN}_{3}$ ). After 5 min incubation on ice, cells were harvested by centrifugation. mRNA extraction, library preparation and RNA sequencing where subsequently performed as described previously in detail (Hauf et al., 2019).

mRNA isolation and RNA sequencing - Shortly, cell pellets were resuspended in $1 \mathrm{ml}$ Lysis Buffer I (25\% sucrose; $20 \mathrm{mM}$ Tris- $\mathrm{HCl} \mathrm{pH} \mathrm{8,0.25} \mathrm{mM} \mathrm{EDTA)} \mathrm{and} 2 \mu \mathrm{l}$ of lysozyme (100 mg ml-1) was added. After $5 \mathrm{~min}$ on ice, the samples were pelleted and resuspended in $300 \mu \mathrm{l}$ Lysis Buffer II ( $3 \mathrm{mM}$ EDTA, $200 \mathrm{mM} \mathrm{NaCl}$ ). This solution was then mixed with $300 \mu$ l Lysis Buffer III ( 3 mM EDTA, $200 \mathrm{mM} \mathrm{NaCl}, 1 \% \mathrm{SDS}$ ) and the mixture was incubated at $95^{\circ} \mathrm{C}$ for 5 more minutes. Six hundred micro liter phenol/chloroform/isoamylalcohol (25:24:1) was added and vigorously mixed at room temperature for $5 \mathrm{~min}$. Phases were separated by centrifugation, the aqueous upper phase was collected and the phenol/chloroform extraction was repeated. Afterward, the aqueous phase was mixed with $600 \mu \mathrm{l}$ chloroform/isoamylalcohol (25:1), shaken vigorously for $5 \mathrm{~min}$ and phases were 
separated by centrifugation. The chloroform extraction was repeated. Finally, RNA was precipitated by addition of $0.1 \times$ volume $3 \mathrm{M}$ sodium acetate $(\mathrm{pH} \mathrm{5.2)}$ and 1.5 volumes $96 \%$ ethanol and incubation at $-20^{\circ} \mathrm{C}$ overnight and pelleted by centrifugation. The pellet was washed with $70 \%$ ethanol and resuspended in $100 \mu \mathrm{l}$ DEPC treated water. Ten micro gram of total RNA were digested with DNAse using the RNase-Free DNase Set (Qiagen). RNA was then purified using RNA clean \& concentrator columns (Zymo Research) for purification for RNA molecules longer than 200 nucleotides. RNA quality was assessed using Agilent Bioanalyzer RNA Nano chips. rRNA depletion was performed using the Ribo-Zero Bacteria Kit (Illumina). A total of approximately $2 \mu \mathrm{g}$ purified RNA was treated with $10 \mu$ l Ribo-ZeroRemoval Solution and RNA concentrations were determined using a Qubit ${ }^{\circledast}$ fluorometer. After rRNA removal, the remaining RNA was pelleted by ethanol precipitation following the recommendations in the Ribo-Zero protocol (Hauf et al., 2019).

RNA libraries were prepared using the TruSeq ${ }^{\circledR}$ Stranded mRNA Kit. The RNA libraries were sequenced in paired-end mode with 2 times 76 cycles on the Illumina MiSeq (MiSeq ${ }^{\circledR}$ Reagent Kit v3; 150 cycles). RNA transcripts were quantified by quasi-mapping of the reads to the L. monocytogenes EGD-e cDNA, provided by the Ensembl Genomes server (Kersey et al., 2018), using the Salmon software (Patro et al., 2017). Average expression from three biological replicates of the mutant divided by the average expression from three biological replicates of the wild type gave the differential expression ratio. $\log _{2^{-}}$transformed transcript counts from three biological replicates were then used to calculate $P$ values using Student's t-test. Significantly differentially expressed genes were defined as having a P-value less than 0.01 and an absolute differential expression factor of more than 2 as well as having an expression level of at least 16 TPM (Hauf et al., 2019).

Protein isolation and proteome analysis - Cell pellets were resuspended in $200 \mu \mathrm{ITT}$ (8 M urea/2 $\mathrm{M}$ thiourea) buffer and immediately released as individual drops directly into a pre-chilled $3 \mathrm{ml}$ Teflon vessel filled with liquid nitrogen. A pre-cooled steel ball ( $8 \mathrm{~mm}$ diameter) was added to the vessel and the frozen cells were mechanically disrupted using the Micro-Dismembrator (Braun, Melsungen) at top speed ( $2600 \mathrm{rpm}$ ) for two minutes. The still frozen powder was instantly taken up in $1800 \mu \mathrm{l}$ UT buffer and then sonified three times for $5 \mathrm{sec}$. at $50 \%$ intensity. Afterwards, the protein extract was centrifuged for $1 \mathrm{~h}$ at $4^{\circ} \mathrm{C}$ and $16000 \mathrm{~g}$. The supernatant was transferred to a fresh tube and the protein concentration was determined with a Bradford assay kit (Pierce, Thermo Scientific, Bonn). Sample aliquots were stored at $-80^{\circ} \mathrm{C}$. Sample aliquots of $2 \mu \mathrm{g}$ were diluted with $20 \mathrm{mM}$ ammonium-bicarbonate $(A B C)$ buffer until a final concentration of less than $1 \mathrm{M}$ urea was reached. Following reduction by $2.5 \mathrm{mM} \mathrm{DTT} \mathrm{(} 30 \mathrm{~min}$ at $37^{\circ} \mathrm{C}$ ) and alkylation by $10 \mathrm{mM}$ lodoacetamide (15 $\mathrm{min}$ at 37 in the dark), trypsin $[20 \mathrm{ng} / \mu \mathrm{l}]$ was added and incubated at $37^{\circ} \mathrm{C}$ overnight. The reaction was stopped with a final concentration of $5 \%$ acetic acid and the peptide mixtures were desalted on $\mathrm{C}-18$ reverse phase material (ZipTip $\mu$-C18 Millipore Corporation, Billerica, MA). Peptides were eluted in 50 and $80 \%$ acetonitrile (ACN) each in $1 \%$ acetic acid. Pooled eluates were dried in a vacuum concentrator and resuspended in $20 \mu \mathrm{l}$ of buffer A (2\% ACN in $0.1 \%$ acetic acid) with a final concentration of $0.1 \mu \mathrm{g} / \mu \mathrm{l}$ protein (tryptic peptides) prior MS analysis.

Mass spectrometry measurements - The tryptic peptides were separated on an Accucore 150-C18 analytical column of $250 \mathrm{~mm}(25 \mathrm{~cm} \times 75 \mu \mathrm{m}, 2,6 \mu \mathrm{m}$ C18 particles, $150 \AA ̊$ pore size, Thermo Fischer Scientific ${ }^{\mathrm{TM}}$, USA) using a Dionex Ultimate 3000 nano-LC system (Thermo Fischer Scientific ${ }^{\mathrm{TM}}$, Germany). The peptide elution was performed at a constant temperature of $40^{\circ} \mathrm{C}$ with a flow rate of $300 \mathrm{~nL} / \mathrm{min}$ with a 60 minutes linear gradient ( $2 \%$ to $25 \%$ ) of buffer (acetonitrile in $0.1 \%$ acetic acid). To design a species-specific spectral library MS/MS data were recorded on a $Q$ Exactive $^{\mathrm{TM}}$ 
HF-X mass spectrometer (Thermo Fischer Scientific ${ }^{\mathrm{TM}}$, Germany) in data-dependent mode (DDA). The DDA-MS measurement parameters are listed in Tab. S4.2. The final MS/MS analyses of samples were performed in data independent mode (DIA) on a Q Exactive ${ }^{\mathrm{TM}} \mathrm{HF}-\mathrm{X}$ mass spectrometer (Thermo Fischer Scientific ${ }^{T M}$, Germany). The DIA-MS measurement parameters are listed in Tab. S4.3.

Analysis of mass spectrometry data - The ion library of L. monocytogenes EGD-e was generated using Spectronaut ${ }^{\mathrm{TM}}$ Pulsar 12 (v12.0.20491.9.26895) software (Biognosys AG, Switzerland). The ion library construction was done using the DDA-MS measurements and the settings summarized in Tab. S4.4. The DIA-MS analysis was also performed using Spectronaut ${ }^{\text {TM }}$ Pulsar 12 with the parameters listed in Tab. S4.5. The DIA-MS data analysis was performed using R (Team, 2014) (version 3.52) and the tidyverse (Vienna, Austria, 2017, n.d.) and PECA (Suomi et al., 2015) package. The raw MS2 total peak area of ions was median normalized on the global median. The median ion quantity was subsequently summed up for each peptide and sample to obtain the peptide intensity. The peptide intensities were used for the statistical ROPECA (reproducibility-optimized peptide change averaging) analysis workflow (Suomi \& Elo, 2017). Proteins with a fold-change $\geq 2$ and $Q$-value $(F D R)<0.05$ were considered as significantly altered in their abundance.

Data analysis - The in gene expression or protein biosynthesis affected genes and proteins were later on analyzed using the Geneious software (Geneious Prime 2019.0.4 (https://www.geneious.com)) and the online resources ListiWiki (http://listiwiki.uni-goettingen.de) and SubtiWiki (http://subtiwiki.uni-goettingen.de), the Listeriomics website (https://listeriomics.pasteur.fr) and the annotation databases RAST and COG (Aziz et al., 2008; Bécavin et al., 2017; Tatusov et al., 2000; Zhu et al., 2018).

\section{Results}

Deletion of cdaA leads to global alteration of gene expression and protein biosynthesis - The L. monocytogenes wild type strain and a clean deletion mutant of the $c d a A$ gene (see chapter 2 ), encoding the sole DAC of L. monocytogenes, were grown under defined conditions in LSM (growth curves are shown in Fig. S4.1) and changes in transcriptome and proteome analyzed (Whiteley et al., 2017). 95 genes were differently expressed (at least a two-fold change and $p<0,01$ ), with 36 down- and 59 upregulated and 21 proteins showed a significant change in abundancy of at least $30 \%$ ( $p$ and $p, f d r<0,01$ ), with 11 showing a lower and 10 a higher abundance (in the $\Delta c d a A$ mutant compared to the wild type strain). Loci of the respective genes and the genes encoding the respective proteins were mapped on the L. monocytogenes EGD-e wt genome. As depicted in Fig. 4.1, differently expressed genes are more or less evenly distributed around the chromosome with some hotspots visible.

Among the most downregulated genes (Tab. 4.4) are genes encoding for proteins involved in cell division (Lmo2506-2507; FtsXE), a putative N-acetylmuramoyl-L-alanine amidase (Lmo1216) and the putative cell wall hydrolase Lmo2522, highlighting the impact of c-di-AMP on cell wall biogenesis and cell wall remodeling. Among others, genes involved in purine biosynthesis (Imo1764Imo1765, Imo1770-Imo1772; purD, purE, purS, purL, purC), in arginine biosynthesis (Imo20902091; $\arg G H)$ and $/ m o 0423$, encoding the in heat stress and cold adaptation involved sigma factor $\sigma^{S}$ are upregulated. This demonstrates the impact c-di-AMP has on central cellular processes, like nucleotide and amino acid biosynthesis and regulation of stress responses. 


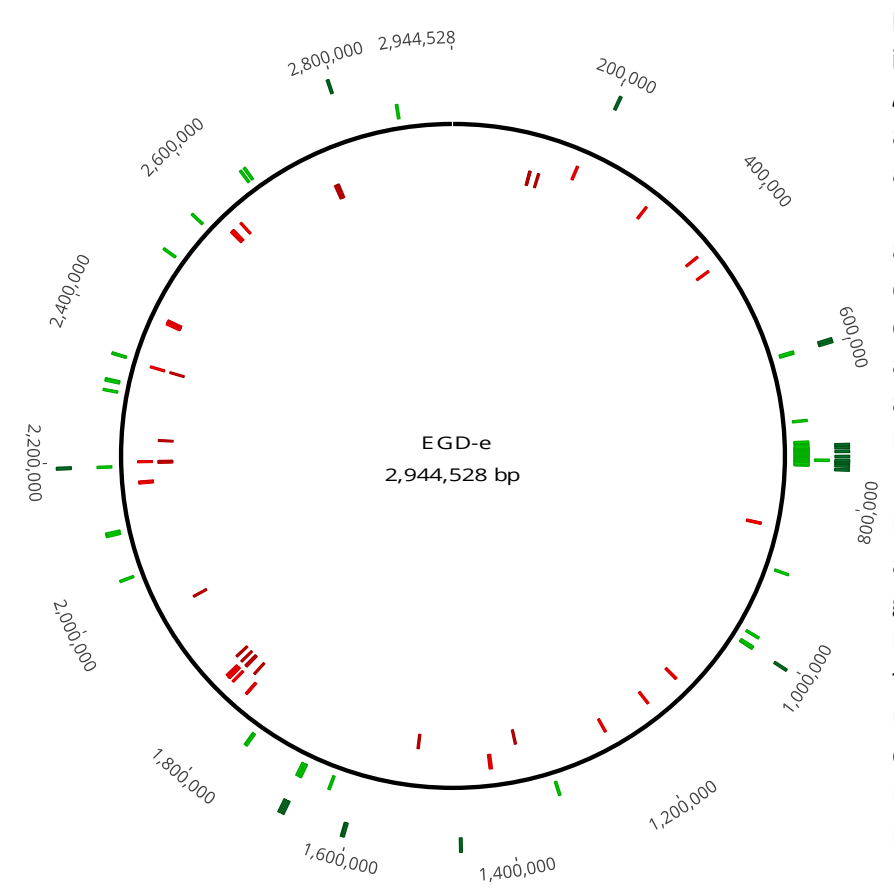

Fig. 4.1 Genome map of $L$. monocytogenes depicting differently expressed genes and proteins in a $\triangle c d a A$ mutant. The L. monocytogenes wt strain and the $\triangle c d a A$ mutant were grown in $L S M$ at $37^{\circ} \mathrm{C}$ and harvested in the exponential growth phase. Differentially expressed genes and proteins were analyzed by RNA-Seq and Mass-spectrometry as described in the experimental procedures. Differentially expressed genes with a $p<0.01$ and at least a two-fold change in expression levels are depicted as light green and light red for up- and downregulated genes, respectively, in the $\Delta c d a A$ mutant. Proteins that show a different abundance with a $p<0,01$ and $a, f d r<0,01$ and a signal to logs ${ }_{2}$ ratio are mapped on their respective gene loci. Dark green and dark red depict proteins with a higher or lower abundance in the $\Delta c d a A$ mutant, respectively. The depiction shows that up- and downregulation of expression is distributed over the whole chromosome with some regions of higher occurrence, e.g. at 3 o'clock.

$\mathrm{p}$, probability value; fdr, false discovery rate.

Tab. 4.4 The twenty most downregulated genes in $\Delta c d a A$

\begin{tabular}{|c|c|c|c|c|}
\hline Locus tag & Operon & Name & RAST info & Fold change \\
\hline Imo2120 & 388 & $c d a A$ & Diadenylate cyclase CdaA & -372.50 \\
\hline Imo1216 & & & $\mathrm{N}$-acetylmuramoyl-L-alanine amidase, family 4 & -17.85 \\
\hline Imo2522 & & yocH & Cell wall-binding protein & -13.04 \\
\hline Imo0186 & & & Cell wall-binding protein & -10.07 \\
\hline Imo1764 & 310 & purD & Phosphoribosylamine--glycine ligase & -4.41 \\
\hline Imo1771 & 310 & purS & Phosphoribosylformylglycinamidine synthase, PurS subunit & -3.86 \\
\hline Imo2506 & 446 & ftsX & Cell division protein FtsX & -3.57 \\
\hline Imo1770 & 310 & purL & $\begin{array}{l}\text { Phosphoribosylformylglycinamidine synthase, glutamine } \\
\text { amidotransferase subunit }\end{array}$ & -3.51 \\
\hline Imo2507 & 446 & ftsE & Cell division transporter, ATP-binding protein FtsE & -3.50 \\
\hline Imo2090 & 381 & $\operatorname{argG}$ & Argininosuccinate synthase & -3.42 \\
\hline Imo1772 & 310 & purC & $\begin{array}{l}\text { Phosphoribosylaminoimidazole-succinocarboxamide syn- } \\
\text { thase }\end{array}$ & -3.37 \\
\hline Imo2254 & & pbuO & $\begin{array}{l}\text { Xanthine/uracil/thiamine/ascorbate permease family pro- } \\
\text { tein }\end{array}$ & -3.22 \\
\hline Imo2344 & 419 & ytnl & glutaredoxin family protein & -3.01 \\
\hline Imo2505 & 446 & spl & Peptidoglycan lytic protein P45 & -2.81 \\
\hline Imo2349 & 419 & tcyk & L-Cystine $A B C$ transporter & -2.76 \\
\hline Imo2091 & 381 & $\operatorname{argH}$ & Argininosuccinate lyase & -2.71 \\
\hline Imo2348 & 419 & tcyl & L-Cystine ABC transporter & -2.69 \\
\hline Imo2345 & 419 & ytmo & $\begin{array}{l}\text { Bacterial luciferase family protein } \mathrm{YtmO} \text {, in cluster with L- } \\
\text { cystine } A B C \text { transporter }\end{array}$ & -2.68 \\
\hline Imo0423 & 74 & & RNA polymerase sigma-70 factor, ECF subfamily & -2.64 \\
\hline Imo1775 & 310 & purE & $\begin{array}{l}\text { Phosphoribosylaminoimidazole carboxylase catalytic subu- } \\
\text { nit }\end{array}$ & -2.64 \\
\hline
\end{tabular}

RAST = Rapid Annotation using Subsystem Technology (database), grey names = homologs of B. subtilis 169 (according to ListiWiki) 
In the absence of c-di-AMP more genes are down- than upregulated and among the most upregulated genes, as shown in Tab. 4.5 are mainly genes involved in motility (see below). Imo2567, encoding together with Imo2568, which was not deregulated, a putative ABC transporter under regulation of the LiaFSR three-component system (Fritsch et al., 2011). This three-component system shows some interesting connections to the c-di-AMP metabolism (see discussion). Two other genes that are more highly expressed in the absence of $c d a A$ are Imo0559 and Imo0560, encoding a putative magnesium and cobalt transporter and the glutamate dehydrogenase, respectively, in L. monocytogenes. Imo0560 is not among the twenty most upregulated genes and can be found with more detailed information and all differentially expressed genes and differently abundant proteins in Tab. S4.1. Interestingly, two sRNAs rli31 and rli32 are located directly in front of Imo0559 and Imo0560, hinting at a regulatory function (see chapter 6).

Tab. 4.5 The twenty most upregulated genes in $\Delta c d a A$

\begin{tabular}{c|c|c|l|c}
\hline Locus tag & Operon & Name & \multicolumn{1}{|c}{ RAST info } & Fold change \\
\hline Imo2567 & & & FlG00774296: hypothetical protein & $\mathbf{1 0 . 8 4}$ \\
Imo0693 & 112 & & Flagellar motor switch protein FliN & $\mathbf{9 . 0 9}$ \\
Imo0691 & 112 & cheY & Chemotaxis regulator & $\mathbf{8 . 9 4}$ \\
Imo0695 & 112 & & hypothetical protein & $\mathbf{8 . 6 6}$ \\
Imo0692 & 112 & cheA & Signal transduction histidine kinase CheA & $\mathbf{8 . 4 3}$ \\
Imo0696 & 112 & flgD & Flagellar basal-body rod modification protein FlgD & $\mathbf{8 . 1 1}$ \\
Imo0694 & 112 & & FlG00774728: hypothetical protein & $\mathbf{7 . 4 4}$ \\
Imo0697 & 112 & flgE & Flagellar hook protein FlgE & $\mathbf{6 . 9 9}$ \\
Imo0699 & 112 & fliM & Flagellar motor switch protein FliM & $\mathbf{6 . 6 9}$ \\
Imo0559 & & & Magnesium and cobalt transport protein CorA & $\mathbf{6 . 6 0}$ \\
Imo0698 & 112 & & Flagellar motor switch protein FliN & $\mathbf{6 . 4 6}$ \\
Imo0974 & 155 & dltA & D-alanine--poly(phosphoribitol) ligase subunit 1 & $\mathbf{6 . 4 0}$ \\
Imo0973 & 155 & dltB & D-alanyl transfer protein DltB & $\mathbf{5 . 7 8}$ \\
Imo0701 & 112 & & FlG00774560: hypothetical protein & $\mathbf{5 . 5 2}$ \\
Imo0702 & 112 & & FlG00774477: hypothetical protein & $\mathbf{5 . 3 8}$ \\
Imo0707 & 112 & flid & Flagellar hook-associated protein FliD & $\mathbf{5 . 3 4}$ \\
Imo0706 & 112 & flgL & Flagellar hook-associated protein FlgL & $\mathbf{5 . 2 5}$ \\
Imo0647 & & & FlG00774323: hypothetical protein & $\mathbf{5 . 2 1}$ \\
Imo0705 & 112 & flgK & Flagellar hook-associated protein FlgK & $\mathbf{5 . 1 5}$ \\
Imo0700 & 112 & & Flagellar motor switch protein FliN & $\mathbf{5 . 0 3}$ \\
\hline RAST = Rapid Annotation using Subsystem Technology (database) &
\end{tabular}

c-di-AMP affects expression of multiple operons - Next, we identified all operons, in which expression of more than one gene was impacted. We identified that eight operons were down- and seven operons were upregulated. The genes Imo1074 and Imo1075 encoding homologs of the essential B. subtilis teichoic acid translocase TagG and TagH, Imo1389-1390 and Imo1738-Imo1739 encoding two $A B C$ transporter of unknown function and putatively for amino acid transport, respectively were identified as downregulated operons. Moreover, nearly the whole operon 419, containing the genes Imo2343-Imo2350 was downregulated (Fig. 4.2). This operon encodes proteins that are homologs of the $B$. subtilis ymt $L$ operon encoding an $A B C$ transporter for cystine uptake and proteins involved in the conversion of S-methyl cysteine to cysteine and thereby involved in amino acid and sulfur metabolism (Burguière, et al., 2005). Other downregulated operons (see Tab. S4.1) 
encode proteins involved in arginine and purine biosynthesis, cell wall biosynthesis and modification of lipo- and wall teichoic acid.

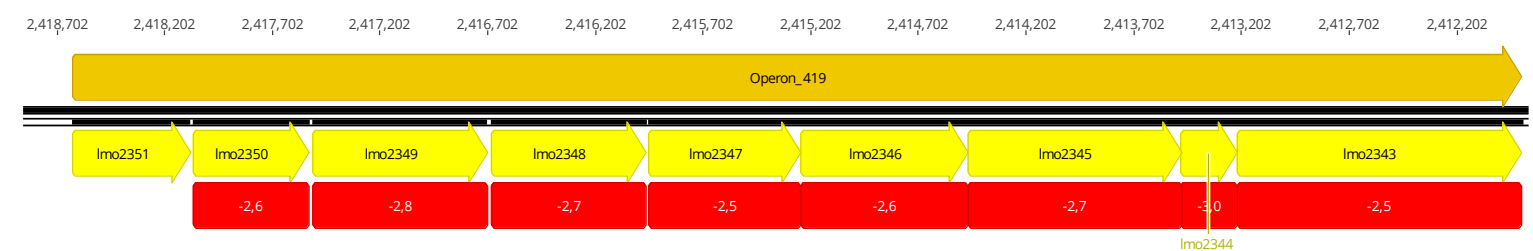

Fig. 4.2 Deletion of $c d a A$ leads to the downregulation of genes of the amino acid metabolism. Depicted is operon 419 of L. monocytogenes EGD-e, consisting of 9 genes (Imo2351-Imo2343). All genes of the operon, except the first one show a 2.5 to 3.0-fold downregulation in the $c d a A$ deletion mutant. The genes in the operon except the not downregulated Imo2351 are homologs of the B. subtilis genes snaA, tcyK, tcyL, tcyM, tcyN, cmoO, cmol and $\mathrm{cmoJ}$ with individual amino acid similarities of the encoded proteins between 65.1 and $77.6 \%$ (ListiWiki). The $B$. subtilis genes encode an $A B C$ transporter for cystine uptake and genes for the conversion of S-methylcysteine to cysteine and are therefore involved in sulfur and amino acid metabolism. The first gene of the operon $I$ mo2351 encodes a protein of unknown function with an FMN reductase domain.

Among the upregulated operons was with operon 281, containing Imo1636 and Imo1637, another $A B C$ transporter encoding operon. Furthermore, operons involved in DNA double strand break repair (Imo2221-Imo2222) and biosynthesis of NAD (Imo2023 and Imo2025) were upregulated, which again highlights a broad influence of c-di-AMP on different cellular processes. Most strikingly however was the upregulation of the operons 111, 112 and 295 as well as other individual genes that all are involved in motility and chemotaxis. As depicted in Fig. 4.3, the flaA gene, encoding the flagellin protein, as well as nearly the whole downstream operon of a size of $23.8 \mathrm{~kb}$ encoding many components of the flagellum was strongly upregulated.

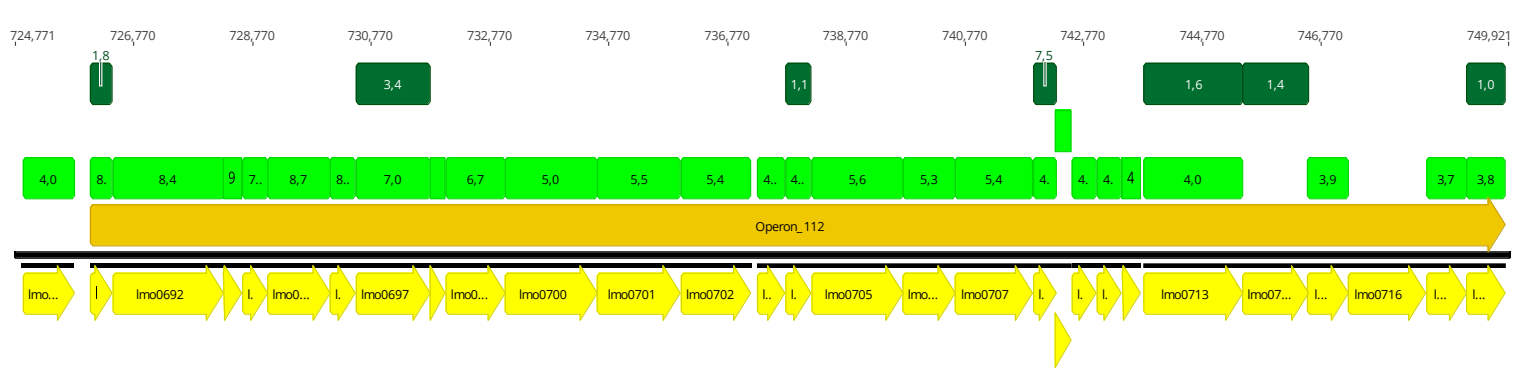

Fig. 4.3 Deletion of $c d a A$ leads to the upregulation of motility associated genes. Depicted is the flaA gene and operon 112 of L. monocytogenes EGD-e. The operon consists of 28 genes (Imo0691-Imo0718). The flagellin encoding flaA and all genes of the operon except fligG, encoding the flagellar basal body protein and flil, encoding a flagellar specific ATPase are 3.7 to 9.1-fold upregulated in the $c d a A$ deletion mutant. Additionally, 7 encoded proteins show a higher abundancy (1-7.5 fold), including FliG.

c-di-AMP impacts synthesis of proteins involved in virulence, antibiotic resistance and motility We also investigated changes in protein expression in the $\triangle c d a A$ strain to find possible regulatory effects on the level of translation, as it has been shown for $y d a O$ mediated regulation of potassium transporter expression in B. subtilis (Gundlach et al., 2017; Nelson et al., 2013). As shown in Tab. 4.6 , the at least 1.5-fold less abundant proteins were the DAC CdaA and its regulator CdaR, which most likely was less abundant due to effects of $c d a A$ deletion on the polycistronic $c d a A-c d a R$ mRNA (ribosomal binding site of $c d a R$ is still present; chapter 2), homologs to the $B$. subtilis PbuO, $\mathrm{Lic} B$ and YefA proteins and the proteins PanB and rpml. Those proteins are putatively involved in transport processes, biosynthesis of coenzyme $A$, rRNA modification and translation. 
Tab. 4.6 At least 1.5-fold downregulated proteins in $\Delta c d a A$

\begin{tabular}{l|c|c|l|c}
\hline Locus tag & Operon & Name & \multicolumn{1}{|c|}{ RAST info } & Fold change \\
\hline Imo2120 & 388 & CdaA & Diadenylate cyclase CdaA & $\mathbf{- 3 . 4 1}$ \\
Imo2119 & 388 & CdaR & Regulator of CdaA activity & $\mathbf{- 3 . 3 5}$ \\
Imo2254 & & PbuO & Xanthine/uracil/thiamine/ascorbate permease family protein & $\mathbf{- 2 . 0 8}$ \\
Imo2683 & 482 & LicB & PTS system, cellobiose-specific IIB component & $\mathbf{- 1 . 9 3}$ \\
Imo1902 & 341 & PanB & 3-methyl-2-oxobutanoate hydroxymethyltransferase & $\mathbf{- 1 . 8 3}$ \\
Imo1751 & 307 & YefA & 23S rRNA (Uracil-5-) -methyltransferase RumA & $\mathbf{- 1 . 7 7}$ \\
Imo1784 & 312 & Rpml & LSU ribosomal protein L35p & $\mathbf{- 1 . 7 1}$ \\
\hline
\end{tabular}

RAST = Rapid Annotation using Subsystem Technology (database), grey names = homologs of B. subtilis 169 (according to ListiWiki)

The at least 1.5-fold more abundant proteins in the $\triangle c d a A$ mutant are listed in Tab. 4.7 (all significantly ( $p$ and $p, f d r<0.01$ ) abundant protein are listed in Tab. S4.1). Among them was the PlcA protein, a phosphatidyl-inositol specific phospholipase that is an important virulence factor of L. monocytogenes for vacuolar escape. Interestingly, the plcA gene (Imo0201) is located in one operon the gene Imo0200 encoding the master virulence regulator PrfA of L. monocytogenes. Expression of prfA and regulation of the ability of PrfA to modulate expression of the virulence genes is quite complex, involving different promoters for prfA expression, regulation by a temperaturedependent riboswitch, modulation of PrfA activity by e.g. glutathione and different affinities of PrfA to the binding regions of genes it regulates (de las Heras et al., 2011; Lobel et al., 2015; Reniere et al., 2015). plcA however is part of the PrfA regulon and therefore also prfA, resulting in a positive feedback loop.

Tab. 4.7 At least 1.5-fold upregulated proteins in $\Delta c d a A$

\begin{tabular}{c|c|c|l|c}
\hline Locus tag & Operon & Name & \multicolumn{1}{|c}{ RAST info } & Fold change \\
\hline Imo0708 & 112 & FliS & Flagellar biosynthesis protein FliS & $\mathbf{7 . 4 5}$ \\
Imo0697 & 112 & FlgE & Flagellar hook protein FlgE & $\mathbf{3 . 4 3}$ \\
Imo0689 & 111 & CheV & Chemotaxis protein CheV & $\mathbf{2 . 1 7}$ \\
Imo0201 & 32 & PlcA & Phosphatidylinositol-specific phospholipase C & $\mathbf{1 . 8 7}$ \\
Imo2115 & 387 & BceB & Bacitracin export permease protein BceB & $\mathbf{1 . 8 7}$ \\
Imo0691 & 112 & CheY & Chemotaxis regulator & $\mathbf{1 . 8 4}$ \\
Imo0713 & 112 & FliF & Flagellar M-ring protein FliF & $\mathbf{1 . 6 3}$ \\
Imo2114 & 387 & YxdL & Bacitracin export ATP-binding protein BceA & $\mathbf{1 . 5 3}$ \\
\hline
\end{tabular}

RAST = Rapid Annotation using Subsystem Technology (database), grey names = homologs of $B$. subtilis 169 (ListiWiki)

Among the other proteins with increased abundance were Lmo2114 and Lmo2115 that are homologs to the $B$. subtilis bacitracin ABC exporter (Clemens et al., 2018), which therefore might also be involved in detoxification and resistance against cell wall acting antibiotics. Interestingly, the genes encoding the ABC transporter, Imo2114 and Imo2115 are in the vicinity of the cdaA-cdaR operon, with about $3 \mathrm{~kb}$ and the genes Imo2116, Imo2115 and the essential $\mathrm{gImM}$ gene that encodes the phosphoglucosamine mutase GImM an enzyme involved in cell wall biosynthesis and regulation of $\mathrm{CdaA}$ activity, between them. As in the transcriptomic analysis, also in the proteomic analysis motility was associated with c-di-AMP. Among the at least 1.5 -fold more abundant proteins were the proteins FliS, FlgE, CheV, CheY and FliF (also see Fig. 4.3). We therefore investigated the motility of the L. monocytogenes wild type, the cdaA deletion mutant, a flil::mgfp (A206K) mutant and deletion mutant of $c d a A, p g p H$ and $p d e A$, with the last three encoding CdaR, the regulator of CdaA and the two PDEs PgpH and PdeA of L. monocytogenes, which degrade c-di-AMP. Strains were assessed on their ability to migrate on LSM or BHI soft agar plates $(0.3 \%(w / v)$ agar $)$ 
at $25^{\circ} \mathrm{C}$ and $37^{\circ} \mathrm{C}$. As shown in Fig. 4.4, the flil mutant was as expected non-motile, the cdaR and $p d e A$ mutants behaved similar to the wild type, whereas the $c d a A$ and $p g p H$ mutant showed a decreased migration radius. L. monocytogenes is usually non-motile at $37^{\circ} \mathrm{C}$, but all strains, except the flil and cdaR mutants showed some, if aberrant, migration with the $c d a A$ mutant having the most uniform spread. Interestingly, on BHI no motility at $37^{\circ} \mathrm{C}$ was observed (data not shown), indicating a temperature-independent regulation of motility.
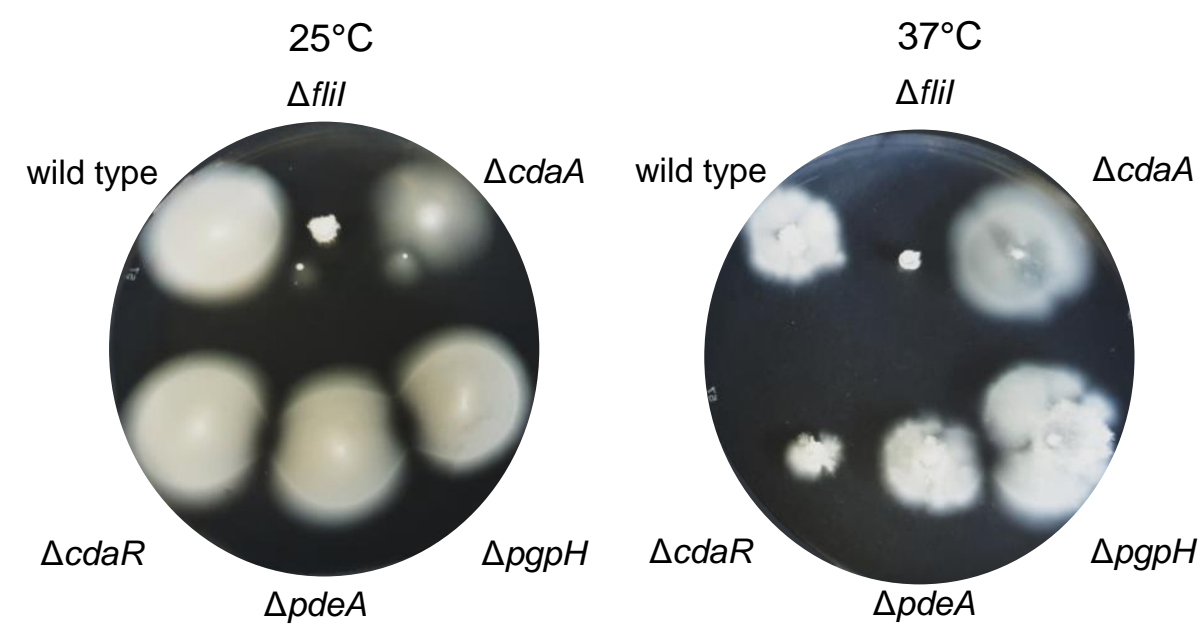

Fig. 4.4 c-di-AMP affects motility of L. monocytogenes. The wild type strain and mutants of $c d a A, c d a R, p d e A, p g p H$ and a $\triangle$ flil::mgfp mutant were investigated for their ability to spread on LSM agar plates. Bacteria were grown in LSM medium and $1 \mu$ l of cells with an $\mathrm{OD}_{600}$ of 1 were stab inoculated in LSM plates containing $0.3 \%(w / v)$ agar. Plates were incubated at $25^{\circ} \mathrm{C}$ and $37^{\circ} \mathrm{C}$ for 3 days.

\section{Discussion}

c-di-AMP influences processes ranging from DNA repair, over cell wall metabolism to the regulation of osmohomeostasis (Corrigan et al., 2011; Gundlach et al., 2017; Luo \& Helmann, 2012; Oppenheimer-Shaanan et al., 2011; Whiteley et al., 2017; Witte et al., 2008; Zeden et al., 2018). We could show that changes in gene expression and protein biosynthesis reflect these phenotypes and present potential novel targets of c-di-AMP signaling on DNA and RNA level. Fig. 4.5 summarizes the differently up- and downregulated genes in functional groups according to the COG database (Tatusov et al., 2009). As shown, most upregulated genes are involved in motility processes or have an unknown function. Among these genes with an unknown function are Imo2567 and Imo2568, encoding a putative ABC transporter. Interestingly, expression of these genes that might comprise a yet not annotated operon, is controlled by the three-component system LiaFSR (Fritsch et al., 2011). The Lia system is known to respond to cell wall-acting peptide antibiotics, like bacitracin and modulate gene expression of lial and liaH that encode proteins important for counteracting cell envelope stress by unknown mechanism. Interestingly the Lia system does not react to antibiotics in cell wall-less $B$. subtilis L-forms and it therefore might not sense antibiotics but their effect on the cell envelope and thereby might act as an indirect cell envelope protection system (Radeck et al., 2017). Moreover, the Lia system is like c-di-AMP signaling conserved amongst firmicutes, which might indicate a functional relationship between those two signal transduction pathways that is further strengthened by the genomic localization of the Imo1023 gene, encoding the c-di-AMP binding protein KtrC, that inhibits c-di-AMP dependent the potassium import of KtrD (see chapter 3). 


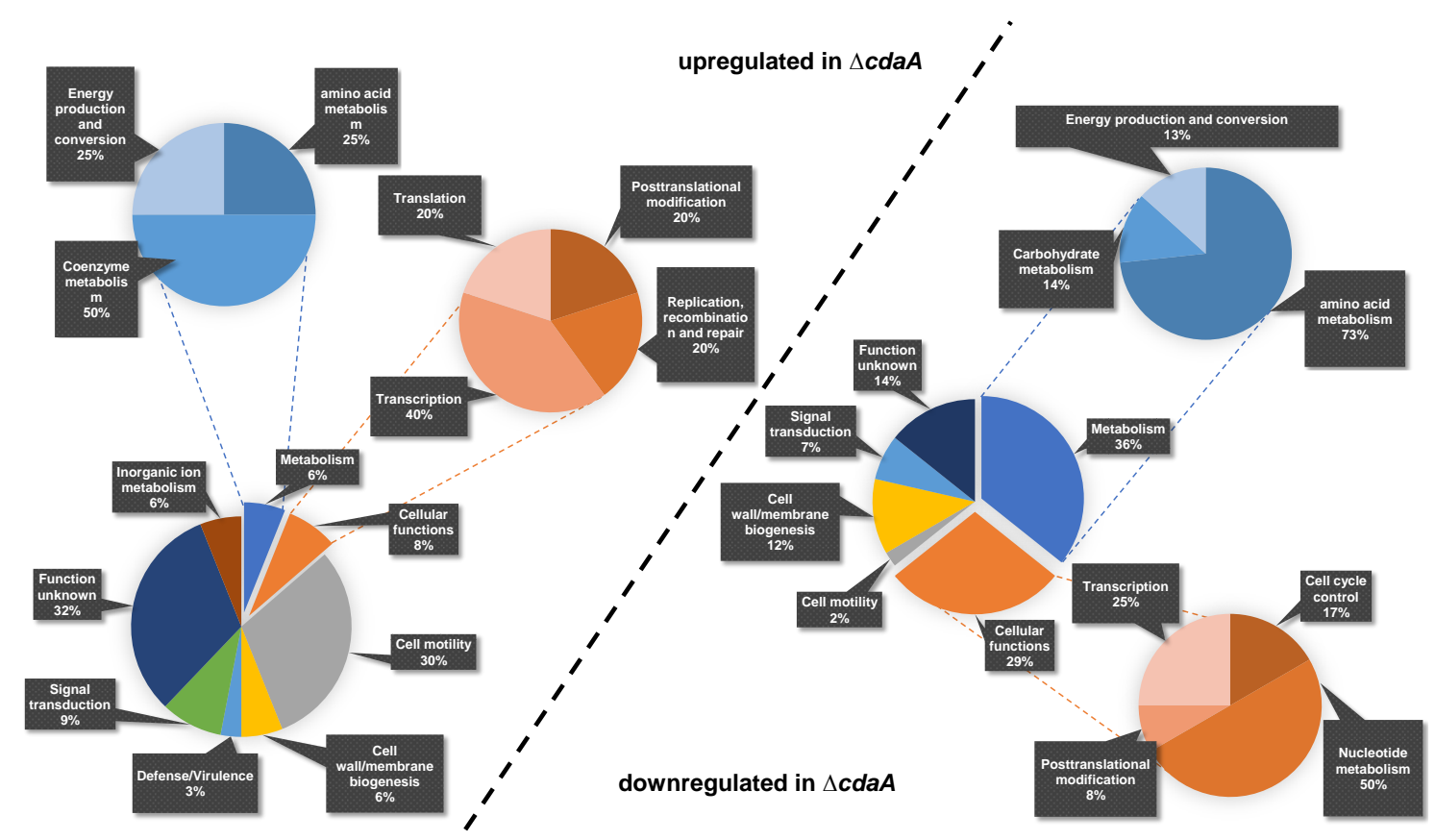

Fig. 4.5 Deletion of $c d a A$ leads to the deregulation of genes involved in all cellular processes. Deletion of the $c d a A$ gene leads to differential expression of 95 genes. 36 genes are down- and 59 are upregulated in the $\Delta c d a A$ mutant compared to the wild type. Down regulated genes encode proteins mainly involved in metabolism, of which the largest part is involved in amino acid metabolism, and proteins involved in cellular functions, such as nucleotide metabolism or transcription. Genes that code for proteins involved in cell wall and membrane biogenesis, signal transduction or with an unknown function make up the remaining third of downregulated genes. The genes that are upregulated in the $\triangle c d a A$ mutant are mainly involved in cell motility or have an unknown function. The remaining third are genes involved in cell wall or membrane biogenesis, signal transduction processes, cellular functions, metabolism, ion transport or virulence associated.

Imo1023 is the last gene in an operon consisting of Imo1018-Imo1022 and Imo1023, with Imo10201022 being liaFSR. Fritsch and colleagues could show that deletion of lias leads to upregulation of the operon, including the $k \operatorname{tr} C$ gene, which might indicate regulation of $k \operatorname{tr} C$ expression in dependency of the LiaFSR three-component system and thereby connecting c-di-AMP regulated potassium import to sensing of cell envelope damage (Fritsch et al., 2011). Interestingly, also the genes Imo2114 and Imo2115, encoding an ABC transporter that are in direct vicinity of the cdaA-cdaR operon are involved in bacitracin resistance and are deregulated in the cdaA mutant. Imo2115 is nearly 4-fold higher expressed in the cdaA mutant and both proteins, Lmo2114 and Lmo2115 have an over 1.5-fold higher abundancy, again highlighting interconnections between c-di-AMP and cell envelope stress responsive systems.

The most striking group of upregulated genes and overproduced proteins are motility associated. It is here interesting to note that in $B$. subtilis a gdpP PDE mutant has a similar phenotype than the cdaA mutant in L. monocytogenes, highlighting that c-di-AMP-dependent regulation of motility might be contrary in those two closely related bacteria (Gundlach et al., 2016). Regulation of motility in L. monocytogenes strongly depends on the ambient temperature. Expression of the main flagellar component, flagellin, is repressed at physiological temperatures $\left(37^{\circ} \mathrm{C}\right)$ and it involves multiple regulatory networks, including the orphan response regulator DegU that binds the promoter of the motB operon encoding among others the gmaR gene (see below) and is therefore essential for synthesis of flagellar components (Dons et al., 1992; Gueriri et al., 2008; Mauder et al., 2008; Peel et al., 1988). Interestingly, DegU is also important in the temperature-dependent expression of flagellar genes, including flaA expression, by antagonizing the transcriptional repressor MogR (Gueriri et al., 2008; Gründling et al., 2004). The ability of MogR to repress gene 
expression is inhibited at $30^{\circ} \mathrm{C}$ and below by the moonlighting protein $\mathrm{GmaR}$ that acts as antirepressor and has furthermore enzymatic activity as a glycosyltransferase that is important for Olinked $\mathrm{N}$-acetylglucosamine modification of flagellin (Shen et al., 2006) Activity of GmaR, however, has no influence on its regulatory properties, which would make GmaR a trigger enzyme (Commichau \& Stülke, 2008; Shen et al., 2006). MogR has been shown to inhibit transcription of flagellar genes at $37^{\circ} \mathrm{C}$, including the GmaR protein. The GmaR protein is a thermosensor protein that changes its conformation upon changes in temperature. At elevated temperatures it is more prone to proteolytic degradation, while on low temperatures it interacts with MogR, is more stable and prevents MogR repression of motility genes. At the same time, DegU acts as a transcriptional activator of GmaR expression in a temperature independent manner, meaning the temperature-dependent phenotype of a degU mutant is only due to the lack of MogR anti-repression by GmaR. If the temperature rises, GmaR-MogR interaction becomes weaker, GmaR is degraded and MogR represses GmaR and other flagellar genes again and the stimulating effect of DegU is not strong enough to overcome the inhibition of gmaR expression by MogR (Kamp \& Higgins, 2008; Kamp \& Higgins, 2011). Interestingly, the 5'-UTR of the mogR mRNA (which is expressional regulated by $\sigma^{\mathrm{B}}$ ) is also an antisense RNA (anti0677) that hybridizes with the mRNA of Imo0675Imo0677, encoding FliNPQ and thereby expression of mogR inhibits translation of the encoded genes (Toledo-Arana et al., 2009). It has also been shown that the genes encoding the flagellar body components FlhB, FliM and FliY are required for expression of other flagellar components and deletion mutant of the respective genes also lead to repression of gene expression of other genes encoding flagellar components in a MogR and GmaR independent matter, suggesting further regulatory mechanisms of expression of flagellar components (Chen et al., 2018). L. monocytogenes possesses also a c-di-GMP signaling network, which in contrast to $B$. subtilis has at least been shown to inhibit motility by regulating the synthesis of a cell aggregation-promoting exopolysaccharide (Chen et al., 2014; Gao et al., 2013; Köseoğlu et al., 2015). It is unknown how c-di-AMP effects expression and biosynthesis of flagellar genes. Neither MogR, nor DegU, nor GmaR seem to be affected, but fliM and fliY are deregulated (together with nearly all genes in operon 112, which makes it unlikely that they are directly affected by c-di-AMP but more likely a consequence of deregulation of the whole operon. We therefore hypothesis that c-di-AMP might have a more direct role in regulation of motility gene expression or indirectly controls yet unknown regulatory mechanisms. The aberrant motility L. monocytogenes shows on LSM minimal medium soft agar plates at $37^{\circ} \mathrm{C}$ might be a hint that also nutrient conditions and thereby altered metabolism and gene expression might influence motility and allow motility at $37^{\circ} \mathrm{C}$. It is, furthermore, interesting to note that the $\mathrm{pgpH}$ mutant showed an inconsistent phenotype with sometimes increased and sometimes decreased motility, whereas the $c d a A$ mutant showed consistently increased motility at $37^{\circ} \mathrm{C}$, but not at $25^{\circ} \mathrm{C}$ (Fig. 4.4). Possible reasons are the slower growth of the cdaA mutant compared to the wild type, which might be more pronounced at $25^{\circ} \mathrm{C}$ or that gene expression might be different, compared to $37^{\circ} \mathrm{C}$. Moreover, it is unknown if the flagellar themselves show an altered activity. There are some indications that c-di-AMP affects the electron motive force, which would also affect ATP synthesis and flagellar activity (Manson et al., 1977; Zeden et al., 2018).

Genes that are downregulated in the $c d a A$ mutant are mainly involved in carbon nitrogen metabolism, nucleotide metabolism and cell wall biogenesis. Most interesting is the lesser abundancy of the KdpD protein (Lmo2681) in the $c d a A$ mutant. The L. monocytogenes KdpABC proteins are homologs to the $S$. aureus potassium importer. In $S$. aureus, the KdpDE two-component system regulates expression of this high affinity potassium transporter. The response regulator KdpE is not phosphorylated upon binding of c-di-AMP to the sensor kinase KdpD and therefore does not 
facilitate $k d p(F) A B C$ in $S$. aureus. Hence, c-di-AMP inhibits upregulation of the $k d p(F) A B C$ genes (Corrigan et al., 2013; Moscoso et al., 2015). Interestingly, we did not see a significant effect on the expression of $k d p A B C$, but it is nevertheless likely that c-di-AMP also acts as an inhibitor of $k d p A B C$ expression in L. monocytogenes and the higher abundancy of $K d p D$ is an indicator that this is indeed the case. There might however be unknown regulatory mechanisms at play. Among the other downregulated processes were pathways involved in arginine, purine and cysteine metabolism that might hint at an imbalance in central metabolic pathways. The higher abundancy of the TCA cycle proteins CitZ, CitB and CitC that are the citrate synthase, aconitate hydratase and isocitrate dehydrogenase of $L$. monocytogenes, respectively, and the ability of c-di-AMP to inhibit the pyruvate carboxylase PycA indicates an involvement of c-di-AMP on regulation of central carbon metabolism, where the TCA cycle is an important intersection (Choi et al., 2017; Sureka et al., 2014; Whiteley et al., 2017). Interestingly the citrate cycle of $L$. monocytogenes is incomplete, due to an absence of the genes encoding the $\alpha$-ketoglutarate $\mathrm{DH}$, succinyl-CoA synthetase and malate dehydrogenase. L. monocytogenes therefore utilizes a glutamate carboxylase to synthesize $\gamma$ amino butyric acid (GABA), which is converted together with $\alpha$-ketoglutarate (the product of $\mathrm{CitC}$ ) by a transaminase to glutamate and succinic semialdehyde (SSA). SSA is subsequently oxidized by a dehydrogenase to succinate which flows back into the TCA cycle (Feehily et al., 2013). The pyruvate carboxylase is hereby the main source of oxaloacetate in L. monocytogenes (Schär et al., 2010). Lack of PycA inhibition and overproduction of CitZBC would therefore lead to an increase flux and lead an increased accumulation of glutamate (Sureka et al., 2014), which could as a consequence lead to an imbalance in amino acid and purine biosynthesis. Whiteley and colleagues could show that citZ mutations restored growth of a $\triangle c d a A$ mutant on complex medium, but interestingly mutations in citB and citC did not phenocopy this effect, which is why the authors hypothesized that accumulation of citrate is toxic for L. monocytogenes lacking c-di-AMP (Whiteley et al., 2017). While it seems established that c-di-AMP regulates the flux of the TCA cycle, it is not yet fully understood which regulatory mechanisms, besides PycA inhibition, contribute to this phenotype. One possibility would be regulation of enzymes involved in the TCA cycle by a c-di-AMP responsive transcriptional modulator. Interestingly, the c-di-AMP binding protein $\mathrm{CbpB}$ is in the same operon with the protein $\mathrm{CcpA}$, which is indeed a transcriptional regulator of the TCA cycle and it is therefore tempting to assume a relationship between c-di-AMP, $\mathrm{CbpB}, \mathrm{CcpC}$ and the upregulation of CitZBC in the $L$. monocytogenes $\triangle c d a A$ mutant.

In summary, we show for the first time, the effect of a deletion of the sole DAC encoding gene cdaA in L. monocytogenes. c-di-AMP effects many cellular processes, ranging from cell wall metabolism, osmoregulation, nitrogen and central carbon metabolism and virulence determinants like motility on the level of gene expression and protein biosynthesis. This study provides interesting new perspectives and highlights potential new regulatory mechanisms of c-di-AMP signaling and will eventually lead to a deeper understanding of the regulatory network.

\section{Acknowledgements}

This work was supported by the grant CO 1139/2-1 from the Deutsche Forschungsgemeinschaft via the Priority Program SPP1879, the Fonds der Chemischen Industrie and the Max-Buchner-Forschungsstiftung (MBFSt-Kennziffer 3381) to FMC. 
Cyclic di-AMP and osmoregulation in Listeria monocytogenes 


\title{
5. The inhibitory effect of DNA on the activity of the di- adenylate cyclase DisA of Bacillus subtilis is relieved by ions
}

Johannes Gibhardt, Anna-Lena Hagemann, Annette Garbe, Volkhard Kaever and Fabian M. Commichau

Author contribution:

$J G$ performed the experiments. $A H, A G$ and $V K$ performed the c-di-AMP measurements. JG and FMC wrote the manuscript.

\begin{abstract}
Signal transduction by nucleotide second messenger molecules is an important tool for bacteria to sense and react to changing environmental conditions or stress conditions. Cyclic di-adenosine monophosphate (c-di-AMP) is unique among bacterial second messenger, as it is both toxic and essential for many bacteria that synthesize it. Synthesis and degradation are performed by diadenylate cyclases (DACs) and specific phosphodiesterases (PDEs), respectively. The soil bacterium Bacillus subtilis possesses three DACs, the sporulation-specific CdaS, the membrane-bound CdaA and the DNA-integrity scanning protein DisA. The DisA cyclase has been shown to bind DNA and move along the chromosome, scanning it for DNA damage and being inhibited in DAC activity upon binding of branched nucleic acids, such as holiday junctions. c-di-AMP has furthermore been shown to regulate potassium and compatible solute homeostasis, which is the reason for its essentiality. We therefore aimed to investigate the role of DisA as a DAC in the context of osmoregulation. We demonstrate that DisA is inhibited by chromosomal DNA (CDNA) in vitro and that this inhibitory effect is counteracted by monovalent cations, such as potassium. We further show that a DisA G334E mutant that has been shown to be less-responsive to branched nucleic acids, is inhibited by cDNA similar to wild type DisA, but the inhibition is more easily counteracted by potassium ions. In vivo, a B. subtilis mutant that expresses DisA wild type or G334E mutant variant, as sole DAC, is able to adapt to osmotic stress. We further show that c-di-AMP concentrations decrease after salt stress with sodium chloride and not with potassium chloride, indicating a discrimination in vivo. We further show that DisA G334E is more active in vivo and beneficial over the wild type variant for adaptation to osmotic stress. In summary, we demonstrate regulation of DisA DAC activity in the context of osmoregulation.
\end{abstract}




\section{Introduction}

Adaptation to changing conditions and danger signals is one of the most important mechanisms in living organisms and facilitated by sophisticated cellular machineries that often involves signal integration by second messenger molecules. An important nucleotide second messenger in bacteria is c-di-AMP (Corrigan \& Gründling, 2013). It has been shown to be involved in many cellular processes, like DNA damage repair, lifestyle changes, cell wall metabolism, regulation of central metabolic processes and regulation of potassium ion and compatible solute homeostasis (Corrigan et al., 2011; Gundlach et al., 2016 Gundlach et al., 2017; Oppenheimer-Shaanan et al., 2011; Sureka et al., 2014; Witte et al., 2008; Whiteley et al., 2017). c-di-AMP is also a unique second messenger, because it is essential in firmicute bacteria, like B. subtilis, Lactococcus lactis, Listeria monocytogenes, or Staphylococcus aureus (Commichau et al., 2015) and furthermore toxic for the bacteria if it accumulates (Gundlach et al., 2015b). It is synthesized by a variety of different DAC domain containing proteins and PDEs (Commichau et al., 2019). In B. subtilis, degradation c-di-AMP is degraded by the PDEs GdpP and PgpH (Gundlach et al., 2015b; Huynh et al., 2015; Mehne et al., 2013; Rao et al., 2010). For the synthesis, B. subtilis expresses the DACs CdaA and DisA, while the third DAC, CdaS, is sporulation-specific expressed (Mehne et al., 2013; Mehne et al., 2014). In other organisms, like the minimal organism Mycoplasma pneumoniae or the archaeon Methanocaldococcus jannaschii, novel types of DACs, CdaM and CdaZ, respectively, have recently been identified, suggesting a widespread conserved function of c-di-AMP in prokaryotes (Blötz et al., 2018; Kellenberger et al., 2015). CdaA interacts and is regulated by the proteins CdaR and the phosphoglucosamine mutase GImM in B. subtilis and related bacteria, like L. monocytogenes, $S$. aureus or L. lactis and is the most abundant type of DAC that is present in many pathogenic firmicutes (Gundlach et al., 2015b; Mehne et al., 2013; Rismondo et al., 2016; Tosi et al., 2019; Zhu et al., 2016). The DisA-type of cyclase is the second most abundant type of DAC and present in spore-forming firmicutes, like B. subtilis or Clostridium difficile, in Actinobacteria the hyperthermophilic bacterium Thermotoga maritima or the Archaeon M. jannaschii (Commichau et al., 2019; Kellenberger et al., 2015; Witte et al., 2018). DisA forms homooctamers, with the monomers consisting of an $\mathrm{N}$-terminal DAC domain that requires $\mathrm{Mg}^{2+}$ as a cofactor and a C-terminal helix-hairpin-helix $(\mathrm{HhH})$ domain that typically bind the phosphate backbone of DNA (Witte et al., 2008). Moreover, DisA strongly binds branched nucleotide substrates that resemble holiday junctions and is inhibited in DAC activity upon binding and a mutation of glycine 334 to glutamate (G334E), a putative DNA-binding residue of DisA, reduced its binding affinity to the branched nucleotide substrates and thereby their inhibitory effect, without an effect on DAC activity in vitro in the absence of DNA (Witte et al., 2008). DisA has also been shown to move as foci along the DNA and in the cytoplasm as a GFP fusion protein and that it is required for the delay of spore formation and germination in response to DNA damage. (Bejerano-Sagie et al., 2006; Campos et al., 2014; Gándara \& Alonso, 2015; Oppenheimer-Shaanan et al., 2011). Moreover, DisA has been shown to inter act in vitro with the DNA segregation-associated protein RacA and the branch migration transferase RadA (Bejerano-Sagie et al., 2006; Ben-Yehuda et al., 2003; Zhang \& He, 2013). Interaction with RadA furthermore inhibits DisA activity in vitro and is expressed in the same operon, but they only seem to co-localize transiently in vivo (Gándara et al., 2017; Zhang \& He, 2013). Intriguingly, c-di-AMP has been shown to be essential due to its regulation of potassium and osmolyte homeostasis in several bacteria, including B. subtilis, L. lactis, L. monocytogenes, S. aureus and Streptococcus agalactiae (Devaux et al., 2018; Gundlach et al., 2017; Pham et al., 2018; Whiteley et al., 2015; Whiteley et al., 2017; Zeden et al., 2018). It even has been proposed that many c-di-AMP associated phenotypes, especially those affecting cell wall-acting antibiotics may be indirectly caused by the impact of c-di-AMP on osmohomeostasis (Commichau et al., 2018). 
Interestingly, it has been shown for $B$. subtilis that none of the DAC encoding genes is essential by itself, rather the presence of c-di-AMP itself. It is sufficient if the CdaS cyclase is expressed with a promoter that is transcribed in vegetative cells (Mehne et al., 2013). Even more so, a mutant with a deletion of all three DAC encoding genes does not show increased occurrence of suppressor mutations or DNA damage repair associated phenotypes, as long as it is grown under osmotically stable and favorable conditions in minimal medium containing a low potassium concentration (Gundlach et al., 2017). We therefore challenged the current model of DisA reporting DNA damage by its DAC activity and investigated the role of DisA in an osmoregulatory context. Using in vitro assays, we show that DisA is inhibited by chromosomal DNA and that inhibition is counteracted by monovalent ions. Furthermore, a G334E mutant that is less inhibited by branched nucleotides in vitro (Witte et al., 2008) is less inhibited by cDNA in the presence of potassium ions and inhibits increased activity in vivo, but not in vitro. Finally, we show that DisA as sole DAC is not only sufficient for survival of $B$. subtilis (Mehne et al., 2013), but also sufficient for $B$. subtilis to adapt to salt stress. Moreover, the G334E mutant has a growth advantage under these conditions, which likely is due to an altered responsiveness in its DNA/ion interaction that affect its DAC activity.

\section{Experimental Procedures}

Bacterial strains and growth conditions $-B$. subtilis 168 and its derivatives were cultivated in LB medium (Sigma-Aldrich) at $37^{\circ} \mathrm{C}$ and $220 \mathrm{rpm}$ if not specified otherwise. Escherichia coli was grown in LB medium at $37^{\circ} \mathrm{C}$ and $220 \mathrm{rpm}$ (strains see Tab. 5.1). As solid medium for $B$. subtilis SP medium was used $\left(8 \mathrm{~g} / \mathrm{l}\right.$ nutrient broth, $0.25 \mathrm{~g} / \mathrm{l} \mathrm{MgSO} \cdot 7 \mathrm{H}_{2} \mathrm{O}, 1 \mathrm{~g} / \mathrm{l} \mathrm{KCl}, 0.5 \mathrm{mM} \mathrm{CaCl}, 10 \mu \mathrm{M} \mathrm{MnCl}$, $4.4 \mathrm{mg} / \mathrm{l}$ iron ferric ammonium citrate). For agar plates, medium was supplemented with $15 \mathrm{~g} / \mathrm{l}$ Bacto Agar (Difco). Antibiotics and medium supplements were used with the following concentrations, if indicated. For B. subtilis erythromycin $(2 \mu \mathrm{g} / \mathrm{ml})$, lincomycin $(25 \mu \mathrm{g} / \mathrm{ml})$, kanamycin $(5 \mu \mathrm{g} / \mathrm{ml})$, chloramphenicol $(5 \mu \mathrm{g} / \mathrm{ml})$ were used and for E. coli ampicillin or carbenicillin $(100 \mu \mathrm{g} / \mathrm{ml})$, chloramphenicol $(30 \mu \mathrm{g} / \mathrm{ml})$, IPTG $(1 \mathrm{mM}$ or $50 \mu \mathrm{M}$; Isopropyl $\beta$-D-1-thiogalactopyranoside; Sigma-Aldrich) and L-arabinose $(0.005 \%(w / v))$ were used.

DNA manipulation - DNA amplification via PCR and transformation of E. coli was performed using standard procedures (Sambrook et al., 1989). DNA fragments were purified using the PCR purification kit (Qiagen) and plasmid DNA was extracted using the NucleoSpin Plasmid Kit (Macherey and Nagel). Commercially available restriction enzymes, T4 DNA ligase and DNA polymerases were used as recommended by the manufacturers. DNA sequences were determined by the dideoxy chain termination method (Microsynth, Göttingen, Germany). Chromosomal DNA of B. subtilis, L. monocytogenes or E. coli were isolated using the NucleoSpin Microbial DNA Kit (Macherey and Nagel). Oligonucleotides were purchased from Sigma-Aldrich (Germany).

Tab. 5.1 Strains

\begin{tabular}{|c|c|c|c|}
\hline Name & Genotype & Description/Construction & Reference \\
\hline \multicolumn{4}{|l|}{ E. coli } \\
\hline BL21(DE3) & 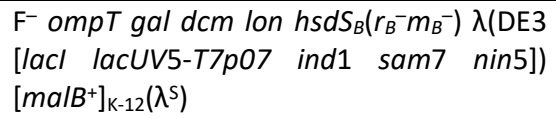 & Protein expression & Stratagene \\
\hline $\begin{array}{l}\text { Rosetta } \\
\text { (DE3) }\end{array}$ & $\begin{array}{l}\mathrm{F}^{-} \quad o m p T h s d S_{B}\left(r_{B^{-}} m_{B^{-}}\right) \text {gal } d c m \text { (DE3) } \\
\text { pRARE (cat) }\end{array}$ & Protein expression & Novagen \\
\hline
\end{tabular}




\begin{tabular}{|c|c|c|c|}
\hline Name & Genotype & Description/Construction & Reference \\
\hline XL1-Blue & $\begin{array}{l}\text { recA1 endA1 gyrA96 thi-1 hsdR17 supE44 } \\
\text { relA1 lac }\left[\mathrm{F}^{\prime} \text { proAB lacla } Z \Delta \mathrm{M} 15 \text { Tn10 }\right. \\
\left.\left(\text { Tet }^{r}\right)\right]\end{array}$ & Cloning & Stratagene \\
\hline \multicolumn{4}{|l|}{ B. subtilis } \\
\hline 168 & $\operatorname{trpC2}$ & Wild type & Laboratory collection \\
\hline GP2032 & $\operatorname{trp} C 2 \Delta c d a S:: e r m C \triangle c d a A:: c a t$ & Deletion of $c d a S$ and $c d a A$ & Gundlach et al., 2015 \\
\hline BP170 & $\operatorname{trpC2} \operatorname{dis} A(\mathrm{wt}) a p h A 3$ & $\operatorname{dis} A-a p h A 3(w t)_{L F H} \rightarrow 168$ & This work \\
\hline BP171 & $\operatorname{trpC2} \operatorname{dis} A(\mathrm{G} 334 \mathrm{E})$ aphA3 & $\operatorname{disA}-a p h A 3(\mathrm{G} 334 \mathrm{E})_{\mathrm{LFH}} \rightarrow 168$ & This work \\
\hline BP172 & $\begin{array}{l}\operatorname{trpC2} \Delta c d a S: \text { ermC } \triangle c d a A:: c a t \text { disA (wt) } \\
\text { aphA3 }\end{array}$ & $\mathrm{BP} 170 \mathrm{cDNA} \rightarrow \mathrm{GP} 2032$ & This work \\
\hline BP173 & $\begin{array}{l}\operatorname{trpC2} \Delta c d a S:: e r m C \quad \Delta c d a A:: c a t \text { disA } \\
\text { (G334E) aphA3 }\end{array}$ & $\mathrm{BP} 171_{\mathrm{CDNA}} \rightarrow \mathrm{GP} 2032$ & This work \\
\hline \multicolumn{4}{|c|}{ L. monocytogenes } \\
\hline EGD-e & Wild type & Serotype $1 / 2$ a strain & Laboratory collection \\
\hline
\end{tabular}

Plasmid construction - For the expression and purification of $\mathrm{N}$-terminal His ${ }_{6}$-tagged DisA (G334E), plasmid pBP392 was constructed as follows (plasmids are listed in Tab. 5.2). The disA gene was amplified from $B$. subtilis CDNA using the oligonucleotide pair FX111/FX112 with the addition of the 5'-phosphorylated oligonucleotide JH106 (oligonucleotides are listed in Tab. 5.3). It introduces the G334E mutation, which reduces inhibition of DisA by branched nucleotides, via the combined chain reaction. The resulting PCR product was digested using $N$ del and $X b a l$ and ligated to $\mathrm{pET} 19 \mathrm{~b}$ that was cut with the same enzymes. (Bi \& Stambrook, 1997; Witte et al., 2008). Plasmids pBP394 and pBP395 allow L-arabinose inducible expression of DisA and DisA (G334E) in E. coli, respectively. The disA wt gene was amplified from $B$. subtilis CDNA and the G334E mutant allele from the plasmid pBP392 using oligonucleotides JH 109 and JH110. The resulting PCR product was digested with $K p n l$ and $X b a l$ and ligated to pBAD33 that was cut with the same enzymes.

Tab. 5.2 Plasmids

\begin{tabular}{|c|c|c|}
\hline Name & Insert/Features & Reference \\
\hline pBAD33 & $\mathrm{P}_{\mathrm{BAD}}$ cat araC & Guzman et al., 1995 \\
\hline pET19b & $\mathrm{P}_{\mathrm{T} 7} \mathrm{~N}-\mathrm{His}_{6}-\mathrm{MCS}$ bla & Novagen \\
\hline pDG670 & MCS aphA3 MCS bla & Guérout-Fleury et al., 1995 \\
\hline pGP172 & $\mathrm{P}_{\mathrm{T} 7} \mathrm{~N}-$ Strep-tag $\|$ bla & Merzbacher et al., 2004 \\
\hline pGP2563 & pET19b-disA & Mehne et al., 2013 \\
\hline pGP2593 & pGP172-cdaS (B. cereus) & Mehne, 2014 \\
\hline pBP392 & pET19b-disA (G334E) & This work \\
\hline pBP394 & pBAD33-disA & This work \\
\hline pBP395 & pBAD33-disA (G334E) & This work \\
\hline
\end{tabular}

bla $=\mathrm{amp}^{\mathrm{R}}\left(100 \mu \mathrm{g} / \mathrm{ml}\right.$ ampicillin or carbenicillin), ermC $=\operatorname{ery}^{\mathrm{R}}(5 \mu \mathrm{g} / \mathrm{ml}$ erythromycin $), c a t=\mathrm{cm}^{\mathrm{R}}(30 \mu \mathrm{g} / \mathrm{ml}$ chloramphenicol $(E$. coli $)), \mathrm{MCS}=\mathrm{multiple}$ cloning site

Transformation of $B$. subtilis - To induce natural competence of $B$. subtilis, cells were overnight in $4 \mathrm{ml} \mathrm{LB}$ medium at $28^{\circ} \mathrm{C}$ and the precultures used to inoculate $10 \mathrm{ml}$ MNGE medium (), supplemented with $0.1 \%(w / v) C A A$ and incubated at $37^{\circ} \mathrm{C}$ and $220 \mathrm{rpm}$ from $\mathrm{OD}_{600} 0.1$ to 1.3 . Cells were diluted 1:1 with pre-warmed MNGE medium, lacking CAA to induce nutrient starvation. Growth was continued for another hour. $400 \mu \mathrm{l}$ of the cells were mixed with DNA and incubated for 30 minutes at $37^{\circ} \mathrm{C}$. Afterwards $100 \mu \mathrm{l}$ of expression mix $(2.5 \%(w / v)$ yeast extract, $2.5 \%(w / v)$ 
CAA and $250 \mu \mathrm{g} / \mathrm{ml}$ tryptophan) was added and the cells incubated for another hour at $37^{\circ} \mathrm{C}$ and 220rpm. Eventually, cells were plated on selective SP agar plates.

B. subtilis strain construction - To investigate the influence of the G334E mutant of DisA in B. subtilis, strains BP170 to BP173 were constructed. About $1 \mathrm{~kb}$ of disA or the disA G334E mutant gene, including its stop codon, were amplified from the $B$. subtilis wt CDNA or pBP392, respectively. The aphA3 gene, encoding the kanamycin resistance, was amplified from pDG670, without promoter or terminator using oligonucleotides JH116 and JH117 (Guérout-Fleury et al., 1995). About $1 \mathrm{~kb}$ downstream of the disA gene was amplified using oligonucleotides $\mathrm{JH} 118$ and $\mathrm{JH} 119$. The three PCR fragments were fused by long-flanking homology PCR as described elsewhere (Wach, 1996). B. subtilis 168 was subsequently transformed with the LFH PCR products to construct BP170 and BP171, containing the wt or G334E mutant allele introduced into the natural disA locus with a downstream apha3 gene integrated into the operon and expression driven by the promoter of the operon. Therefore, selection was performed using 5 instead of $10 \mu \mathrm{g} / \mathrm{ml}$ kanamycin and it was only used as selection marker on plates and in the pre-cultures to prevent an effect of selective pressure of aphA3 expression on disA expression. Correct sequences were confirmed by sanger sequencing. Strains BP172 and BP173 were constructed by transformation of the $c d a A$ and $c d a S$ mutant GP2032, with the CDNA of strains BP172 and BP173, respectively. The correct sequence of the disA locus and absence of $c d a A$ and $c d a S$ was verified by sequencing and PCRs, respectively. BP172 and BP173 express DisA wt or the G334E mutant as sole DACs, respectively.

Tab. 5.3 Oligonucleotides

\begin{tabular}{|c|c|c|}
\hline Name & $\begin{array}{l}\text { Restriction sites are underlined, complementary regions or mutations are in } \\
\text { bold, sequences } 5^{\prime} \rightarrow 3^{\prime}\end{array}$ & Purpose and reference \\
\hline FX111 & AAACATATGGAAAAAGAGAAAAAAGGGGCGAAACACG & $\begin{array}{l}\text { Fwd. disA (Ndel), Mehne } \\
\text { et al., } 2013\end{array}$ \\
\hline FX112 & TTTGGATCCTCACAGTTGTCTGTCTAAATAATGCTTCTCTTG & $\begin{array}{l}\text { Rev. disA (BamHI), Mehne } \\
\text { et al., } 2013 \\
\text { Fwd. B. subtilis ktrAB (E- }\end{array}$ \\
\hline $\mathrm{JH} 53$ & AAAGAATTCAAGGGAGATATGAACATTGGGAAGAATTAAAAATAAG & $\begin{array}{l}\text { coRI), Gundlach et al., } \\
2017\end{array}$ \\
\hline $\mathrm{JH} 54$ & TTTGGATCCTCACCCTGTAAACACTTCGCCATCA & $\begin{array}{l}\text { Rev. B. subtilis ktrAB } \\
(\text { BamHI), Gundlach et al., } \\
2017\end{array}$ \\
\hline JH106 & P-GGCGAGTGCAGAAGAATTAGATGAAGTAGAGGAAATCGGTGAAGTACGAGCCC & $\begin{array}{l}\text { 5'-phosphorylated } \\
\text { GGA } \rightarrow \text { GAA (DisA G334E) }\end{array}$ \\
\hline JH109 & AAAGGTACCTTAGGAGGATAATAGATGGAAAAAGAGAAAAAAGGG & Fwd. RBS $_{\text {disA }}-$ disA (Kpnl) \\
\hline $\mathrm{JH} 110$ & TTTTCTAGATCACAGTTGTCTGTCTAAATAATGCTTCTCTTGC & Rev. $\operatorname{dis} A(X b a l)$ \\
\hline JH114 & CTGTCATCTATATTGCAGTTTGTTGCTCCG & Fwd. disA (intragenic, LFH) \\
\hline $\mathrm{JH} 115$ & ССTTCCTCTGAAGATGTGTCACAGTTGTCTGTCTAAATAATGCTTCTCTTGC & Rev. $\operatorname{dis} A$ (LFH) \\
\hline JH116 & $\begin{array}{l}\text { CAACTGTGACACATCTTCAGAGGAAGGAAATAATAAATGGCTAAAATGAGAA- } \\
\text { TATCAC }\end{array}$ & $\begin{array}{l}\text { Fwd. } \mathrm{RBS}_{a p h A 3}-a p h a 3(\mathrm{w} / \mathrm{o} \\
\text { promoter) }\end{array}$ \\
\hline JH117 & $\begin{array}{l}\text { TAACCGAAACGCGAAGATGTGCTAAAACAATTCATCCAGTAAAATATAA- } \\
\text { TATTTTATTTTCTCCCAATC }\end{array}$ & $\begin{array}{l}\text { Rev. apha3 (w/o termina- } \\
\text { tor) }\end{array}$ \\
\hline JH118 & $\begin{array}{l}\text { TTGTTTTAACACATCTTCGCGTTTCGGTTAAAACCTTATGAATACGGG- } \\
\text { TATATTAATGTTG }\end{array}$ & $\begin{array}{l}\text { Fwd. disA downstream re- } \\
\text { gion, } \mathrm{LFH} \text { ) }\end{array}$ \\
\hline JH119 & CAGGAAGCACAACAGGCTTAACCGCATTGG & $\begin{array}{l}\text { Rev. disA downstream re- } \\
\text { gion, } \mathrm{LFH} \text { ) }\end{array}$ \\
\hline
\end{tabular}

Protein production, purification and dialysis - To assess DAC activity, E. coli BL21 was transformed with plasmids pGP2563 (pET19b-disA) and pGP2593 (pGP172-cdas ${ }^{\mathrm{Bce}}$ ). For expression of DisA G334E (PBP392) E. coli Rosetta (DE3) was used, since expression in BL21 was suboptimal. Bacteria 
were cultivated overnight in $20 \mathrm{ml} \mathrm{BHI}$ medium (Sigma-Aldrich) at $28^{\circ} \mathrm{C}$ and $220 \mathrm{rpm}$ and precultures used to inoculate 1 I BHI medium containing the appropriate antibiotics, $0.5 \%(\mathrm{w} / \mathrm{v})$ glycerol and $50 \mu \mathrm{l}$ Antifoam Y-30 (Sigma-Aldrich) in $2 \mathrm{I}$ baffled flasks to an $\mathrm{OD}_{600}$ of 0.1 . Bacteria were incubated at $37^{\circ} \mathrm{C}$ and $220 \mathrm{rpm}$ until an $\mathrm{OD}_{600}$ of 0.8-1.2 and protein expression induced with $1 \mathrm{mM}$ IPTG. Growth was resumed for another 3 hours and bacteria harvested by centrifugation. Bacterial cells were disrupted in $15 \mathrm{ml}$ of the buffers ZAP (50 mM Tris- $\mathrm{HCl} \mathrm{pH7.5,} 200 \mathrm{mM} \mathrm{NaCl}$ ) or buffer W (100 mM Tris-HCl pH 8, $150 \mathrm{mM} \mathrm{NaCl}, 1 \mathrm{mM}$ EDTA) with the addition of $15 \mu \mathrm{l}$ DNasel

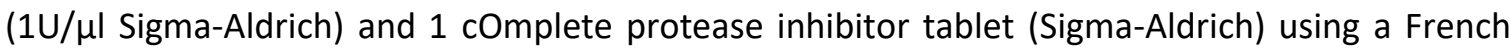
press system with 18000 psi. Cell disruption and purification of the His- and Strep-tagged proteins was performed using $\mathrm{Ni}^{2+}$-NTA and Streptavidin columns (IBA, Göttingen) with imidazole or desthiobiotin (Sigma-Aldrich), as previously described (Mehne et al., 2014; Rosenberg et al., 2015). Protein purification was assessed using coomassie-stained SDS-PAGE (Sambrook et al., 1989) and pooled fractions of enriched protein of interest were dialyzed overnight in the 1000-fold volume at $4^{\circ} \mathrm{C}$ in $10 \mathrm{mM}$ Tris- $\mathrm{HCl} \mathrm{pH} \mathrm{7.5,5 \% (w/v)} \mathrm{glycerol} \mathrm{and} 1 \mathrm{mM}$ 1,4-dithiothreitol (DTT, Sigma-Aldrich), to have the DACs in a salt-free environment.

In vitro DAC assays $-100 \mathrm{nM}$ of freshly dialyzed DACs were incubated in $10 \mathrm{mM}$ Tris $\mathrm{pH} 7.5$ with $0.1 \%(w / v)$ bovine serum albumin, $100 \mu \mathrm{M}$ ATP, $10 \mathrm{mM} \mathrm{MgCl}_{2}$ in a total volume of $200 \mu \mathrm{l}$ at $37^{\circ} \mathrm{C}$ for the indicated time frame. Reactions were stopped by freezing the reaction tubes in liquid nitrogen and subsequent heated for $10 \mathrm{~min}$ at $95^{\circ} \mathrm{C}$, followed by the extraction of c-di-AMP (see next part). For assays where DNA, ions or both were added, the reactions were stopped after 30 min. If indicated, chromosomal DNA of $B$. subtilis 168 , E. coli XL-1blue or L. monocytogenes EGDe or PCR product of $B$. subtilis $k t r A B(\mathrm{JH} 53 / \mathrm{JH} 54)$ was added in the indicated concentration. The additives $\mathrm{NaCl}, \mathrm{KCl}$, sucrose or potassium phosphate were added as indicated. The potassium phosphate solution was prepared by titration of $1 \mathrm{M} \mathrm{KH}_{2} \mathrm{PO}_{4}$ to $1 \mathrm{M} \mathrm{K}_{2} \mathrm{HPO}_{4}$ (approximately 4 to 1 ratio) to $\mathrm{pH} 7.5$ at $37^{\circ} \mathrm{C}$ and dilution to $0.55 \mathrm{M}$ that equals a molarity of $1 \mathrm{M}$ potassium in the solution.

Determination of the c-di-AMP concentration - The stopped reactions were centrifuged for $10 \mathrm{~min}$ at $20000 \mathrm{~g}$ and $4^{\circ} \mathrm{C}$ after the heating step and $175 \mu \mathrm{l}$ of the supernatant was added to $800 \mu \mathrm{l} \mathrm{ex}-$ traction mix (acetonitril:methanol (1:1)) and incubated overnight at $-20^{\circ} \mathrm{C}$. Afterwards the mixtures were centrifuged again and the supernatants were transferred to new tubes and evaporated using SpeedVac at $60^{\circ} \mathrm{C}$ for about $2 \mathrm{~h}$. Extract pellets were resuspended in $200 \mu \mathrm{l} \mathrm{H}_{2} \mathrm{O}$ and ${ }^{13} \mathrm{C},{ }^{15} \mathrm{~N}$ c-di-AMP as internal standard added and the samples analyzed by LC-MS/MS, as described elsewhere (Rismondo et al., 2016).

Salt shock and determination of the intracellular c-di-AMP concentration - B. subtilis strains BP172 and BP173 were grown in $10 \mathrm{ml} \mathrm{LB}$ medium with the appropriate antibiotics overnight at $28^{\circ} \mathrm{C}$ and $220 \mathrm{rpm}$. Main cultures (100 ml LB) were inoculated to an $\mathrm{OD}_{600}$ of 0.05 and incubated at $37^{\circ} \mathrm{C}$ and $220 \mathrm{rpm}$. When cells reached the exponential growth phase $\left(\mathrm{OD}_{600} 0.5 \pm 0.1\right)$, samples were taken $\left(t_{0}\right)$ and the salt shock induced as follows. $25 \mathrm{ml}$ culture were added to $25 \mathrm{ml}$ pre-warmed LB medium without osmolytes or with $\mathrm{NaCl}$ or $\mathrm{KCl}$ to reach a final concentration of $1.5 \mathrm{M}$ salt. Growth was continued and additional samples taken $30 \mathrm{~min}\left(\mathrm{t}_{1}\right)$ and $3 \mathrm{~h}\left(\mathrm{t}_{2}\right)$ after the salt stress. Determination of the intracellular c-di-AMP concentration in DAC expressing $E$. coli was performed similar. E. coli XL1-blue harboring plasmids pBAD33 or the derivatives pBP394 or pBP395 for the expression of DisA or DisA G334E, respectively, were grown overnight in $5 \mathrm{ml}$ LB with $0.02 \%(w / v) \mathrm{D}$ glucose at $28^{\circ} \mathrm{C}$ and $220 \mathrm{rpm}$. Precultures were used to inoculate $50 \mathrm{ml} \mathrm{LB}$ medium with to an $\mathrm{OD}_{600}$ of 0.05 and bacteria grown until the early exponential phase $\left(\mathrm{OD}_{600} 0.2 \pm 0.05\right)$ and expression of DACs was induced by addition of $0.005 \%(w / v)$ L-arabinose. After an additional 1.5 hours samples 
were taken to determine the protein and c-di-AMP concentrations. Sampling, cell disruption, c-di-AMP extract, measuring of the c-di-AMP concentration and normalization to the protein concentration were exactly performed as previously described (chapter 2; Rismondo et al., 2016). To assess the growth behavior after osmotic stress, bacteria were incubated as described above, or using the Synergy Mx multiwell platereader, equipped with the Gen5 software (02.09.2001, BioTek Instruments) Cultures were handled the similar, but the exponential growing overday cultures were adjusted to an $\mathrm{OD}_{600}$ of 0.1 in LB and $200 \mu$ added to the wells of a 96-well plate (Microtest Plate $96-$ Well,F, Sarstedt). Bacteria were incubated for 1.25 hours at $37^{\circ} \mathrm{C}$ with medium shaking and the $\mathrm{OD}_{600}$ measured in $15 \mathrm{~min}$ intervals. Subsequently, either $200 \mu \mathrm{l}$ of the cultures were transferred to empty wells or $100 \mu$ added to LB medium, or to $100 \mu \mathrm{l} \mathrm{LB}$ medium containing $3 \mathrm{M}$ $\mathrm{NaCl}, \mathrm{KCl}$ or sucrose, to obtain a final osmolyte concentration of $1.5 \mathrm{M}$, respectively. Eventually, growth was resumed using the same settings.

\section{Results}

DisA is inhibited by chromosomal DNA - The DAC cyclase DisA, with an N-terminal His 6 -tag, was purified from $E$. coli and dialyzed overnight in salt-free buffer. DisA was incubated with its cofactor $\mathrm{Mg}_{2+}$ and its substrate ATP, as described in the experimental procedures and c-di-AMP extracted after different time points. As shown in Fig. 5.1, A, the enzyme shows a similar activity in the first $120 \mathrm{~min}$ and we therefore performed all subsequent DAC assays for $30 \mathrm{~min}$.

Next, we assessed the ability of chromosomal DNA to inhibit DAC activity of DisA. Therefore, we incubated DisA with different amounts of $B$. subtilis CDNA and also tested the impact of CDNAs from other bacteria, like E. coli or L. monocytogenes. Furthermore, we analyzed if cDNA has an effect on DAC activity of the sporulation-specific DAC CdaS from $B$. subtilis, which was purified via Strep-tag purification. As expected, DAC activity of CdaS was not influenced by CDNA, while DisA was strongly inhibited in a concentration dependent matter and inhibition was independent of the cDNA donor organism (Fig. 5.1, B).

A

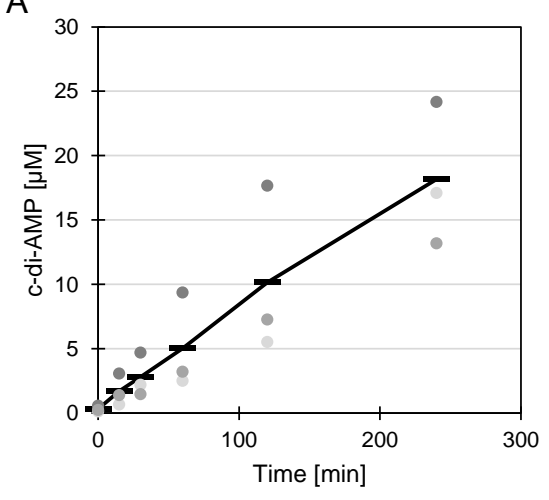

B

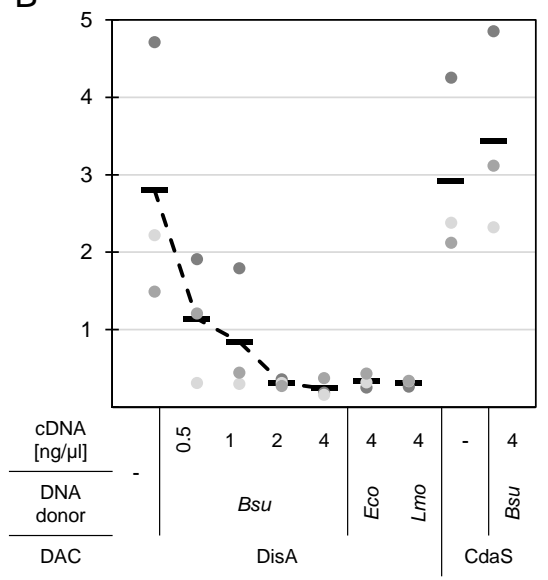

Fig. 5.1 DisA is inhibited by chromosomal DNA. The DACs DisA of $B$. subtilis and CdaS of B. cereus were purified via Hisand Strep-tag purification, respectively. Proteins were dialyzed overnight in $10 \mathrm{mM}$ Tris- $\mathrm{HCl} \mathrm{pH} \mathrm{7.5,5 \% (w/v)} \mathrm{glycerol}$ and $1 \mathrm{mM}$ DTT at $4^{\circ} \mathrm{C} .100 \mathrm{nM}$ of DAC was incubated in $10 \mathrm{mM}$ Tris- $\mathrm{HCl} \mathrm{pH} 7.5$ with $100 \mu \mathrm{M} \mathrm{ATP}, 10 \mathrm{mM} \mathrm{MgCl}$ and 0.1 $\%(w / v) B S A$ at $37^{\circ} \mathrm{C}$. (A) DisA activity over time. Reactions were stopped after the indicated time points and c-di-AMP extracted. (B) Impact of cDNA on DAC activity. DisA or CdaS were incubated without or with the indicated concentrations of cDNA. The reactions were stopped after $30 \mathrm{~min}$ of incubation and c-di-AMP was extracted. Data of three biological replicates are shown as the mean of two technical replicates per measurement point. Horizontal lines depict the mean of the three biological replicates. $c D N A=$ chromosomal DNA, Bsu $=B$. subtilis, $E c o=E$. coli, Lmo $=L$. monocytogenes. 
Addition of ions counteract the inhibitory effect of CDNA - Next, we wanted to analyze if osmolytes have an effect on the inhibitory effect of CDNA. We therefore incubated DisA with or without $1 \mathrm{ng} / \mu \mathrm{l}$ B. subtilis cDNA, as indicated, and added $0,1,10,50$ or $100 \mathrm{mM}$ of the osmolytes $\mathrm{KCl}, \mathrm{NaCl}$ or sucrose to the mixture and analyzed c-di-AMP synthesize after $30 \mathrm{~min}$ of incubation. As depicted in Fig. 5.2, addition of osmolytes does not influence DAC activity of DisA in the absence of DNA. Furthermore, in the presence of cDNA, the non-ionic osmolyte sucrose has no effect on DisA activity, but the ionic osmolytes $\mathrm{KCl}$ and $\mathrm{NaCl}$ counteract the inhibitory effect of cDNA at concentrations greater than $10 \mathrm{mM}$. We also tested if double stranded DNA (dsDNA) has an impact on DisA activity in our assay conditions and incubated DisA with either 0.25 or $1 \mathrm{ng} / \mu \mathrm{l}$ of a $2 \mathrm{~kb} P C R$ product of the $B$. subtilis $k t r A B$ operon, with or without $50 \mathrm{mM}$ of $\mathrm{KCl}$ and compared the effects to the effect of cDNA. While we could observe an effect of dsDNA on DisA activity and higher activity if potassium ions were present, but the effect was not as pronounced, as with cDNA and we therefore continued the experiments using CDNA (Fig. S5.1).

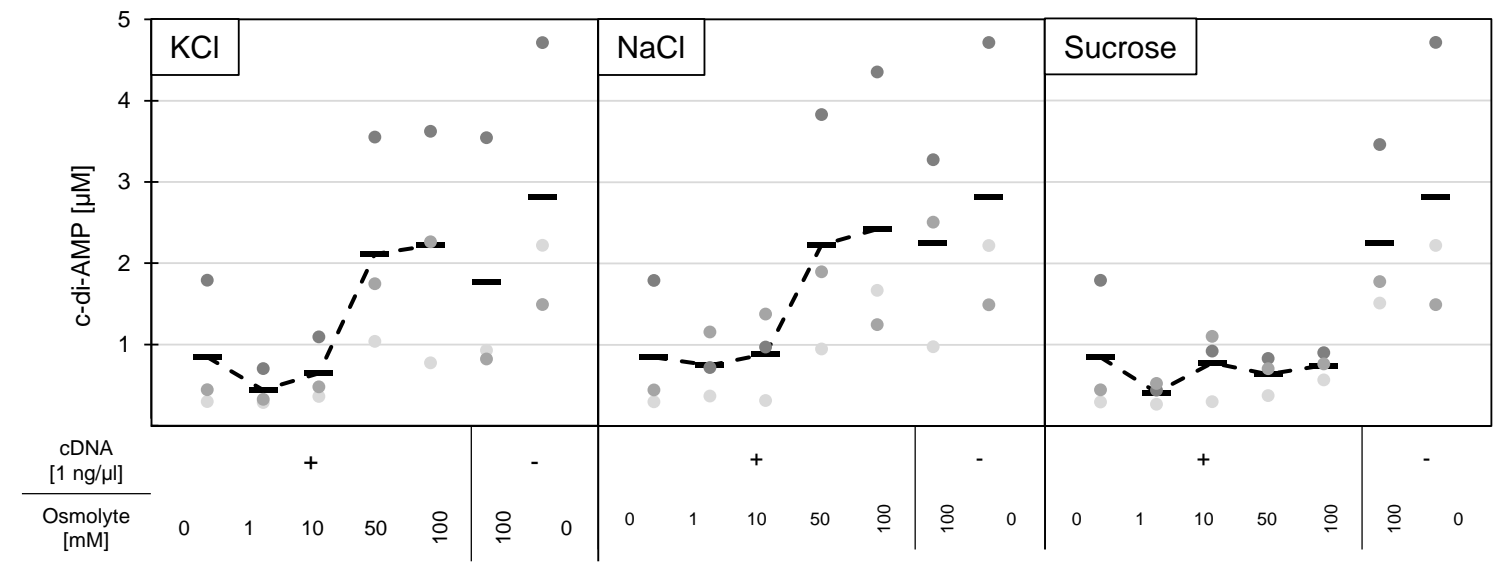

Fig. 5.2 DNA-dependent inhibition of DisA is counteracted by monovalent ions. The DACs DisA of $B$. subtilis was purified via His-tag purification and subsequently dialyzed overnight in $10 \mathrm{mM}$ Tris- $\mathrm{HCl} \mathrm{pH} \mathrm{7.5,5 \% (w/v)} \mathrm{glycerol} \mathrm{and}$ $1 \mathrm{mM}$ DTT at $4^{\circ} \mathrm{C} .100 \mathrm{nM}$ of DisA was incubated in $10 \mathrm{mM}$ Tris- $\mathrm{HCl} \mathrm{pH} 7.5$ with $100 \mu \mathrm{M} \mathrm{ATP}, 10 \mathrm{mM} \mathrm{MgCl}$ and $0.1 \%$ $(w / v)$ BSA at $37^{\circ} \mathrm{C}$ for $30 \mathrm{~min}$ when the reactions were stopped and c-di-AMP extracted. Samples were incubated with or without $1 \mathrm{ng} / \mu \mathrm{l}$ of $B$. subtilis CDNA and with or without the indicated concentration of the two ionic osmolytes $\mathrm{NaCl}$ or $\mathrm{KCl}$ or the non-ionic osmolyte sucrose. Data of three biological replicates are shown as the mean of two technical replicates per measurement point. Horizontal lines depict the mean of the three biological replicates. $c D N A=$ chromosomal DNA.

DisA G334E mutant shows similar activity and inhibition by CDNA in vitro - To assess whether similar effects can be observed for DisA with an altered DNA-binding, we purified and dialyzed DisA with an amino acid exchange of glycine 334 to glutamate (G334E), which has been shown to have a decreased binding capability to branched nucleotides and to be less inhibited by them (Witte et al., 2008). We first analyzed c-di-AMP formation over time and compared it to the wild type DisA. As described by Witte and colleagues, DisA G334E has the same activity as the wild type enzyme in vitro (Witte et al., 2008; Fig. 5.3, A). Next, we assessed the effect of cDNA of DisA G334E activity and observed a similar inhibitory effect of cDNA on the mutant as we saw for the wild type enzyme (Fig. 5.3, B). Therefore, we compared the effects of addition of potassium ions on the activity of the mutant enzyme. 
A

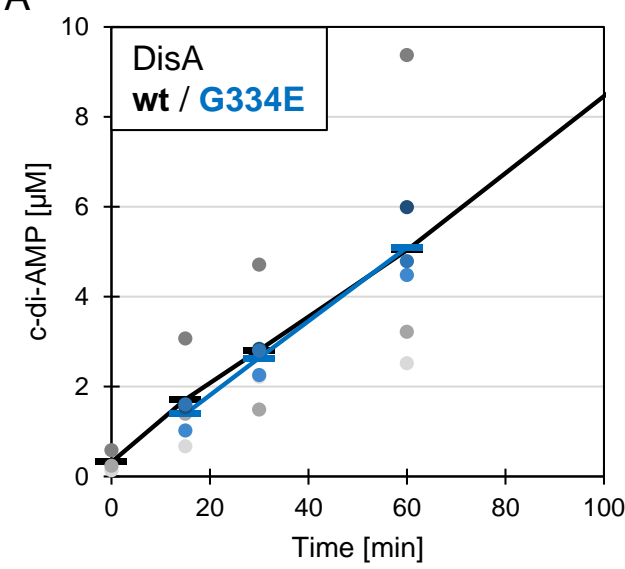

B

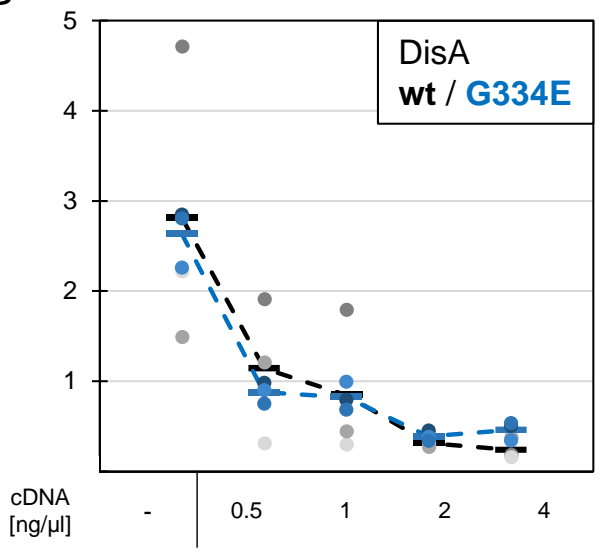

Fig. 5.3 DisA G334E is also inhibited by chromosomal DNA in vitro. The G334E mutant of $B$. subtilis DisA was purified via His-tag purification and subsequently dialyzed overnight in $10 \mathrm{mM}$ Tris- $\mathrm{HCl} \mathrm{pH} \mathrm{7.5,5 \% (w/v)} \mathrm{glycerol} \mathrm{and} 1 \mathrm{mM}$ DTT at $4^{\circ} \mathrm{C} .100 \mathrm{nM}$ of DisA G334E was incubated in $10 \mathrm{mM}$ Tris- $\mathrm{HCl} \mathrm{pH} 7.5$ with $100 \mu \mathrm{M} \mathrm{ATP}, 10 \mathrm{mM} \mathrm{MgCl}$ and $0.1 \%(w / v)$ $\mathrm{BSA}$ at $37^{\circ} \mathrm{C}$. (A) DisA G334E activity over time. Reactions were stopped after the indicated time points and c-di-AMP extracted. Data of the mutant in red is overlaid on the measurements previously obtained for wt DisA in black, as a comparison. (B) Impact of CDNA on DAC activity of DisA G334E. The DAC was incubated without or with the indicated concentrations of $B$. subtilis CDNA. The reactions were stopped after $30 \mathrm{~min}$ of incubation and c-di-AMP was extracted. Data of three biological replicates are shown as the mean of two technical replicates per measurement point. Horizontal lines depict the mean of the three biological replicates. $c D N A=$ chromosomal DNA.

As shown in Fig. 5.4, addition of potassium does not increase activity of DisA G334E in the absence of CDNA, only in the presence of it. Moreover, the inhibition of DAC activity seems to be slightly better counteracted by potassium ions at lower concentrations, compared to the wild type, with similar activities at higher concentrations. Eventually, we also assessed the DAC activities of DisA wild type and G334E mutant in vivo, to see whether the mutation might have an effect under more natural conditions. Indeed, we observed higher activity of DisA G334E, compared to the wild type enzyme (about 1.5-fold), if expressed in E. coli, which does not possess c-di-AMP (Fig. S5.2). This suggests that the mutant has a higher activity in vivo, but not in vitro.

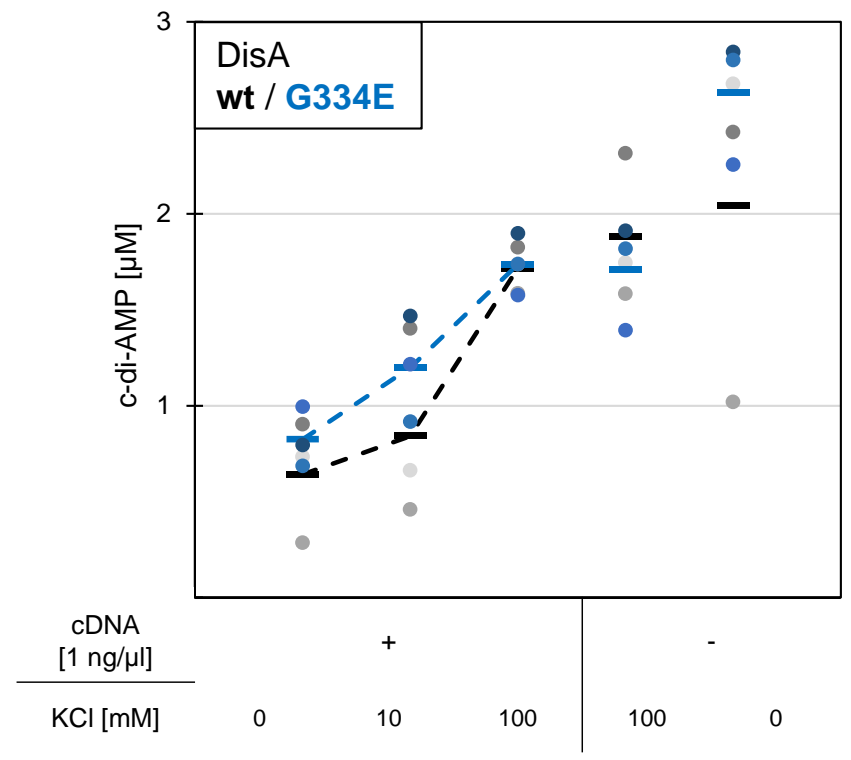

Fig. 5.4 DNA-dependent inhibition of DisA G334E is released at lower potassium concentrations than wild type DisA. The $B$. subtilis DisA wild type and G334E mutant were purified via His-tag purification and subsequently dialyzed overnight in $10 \mathrm{mM}$ Tris- $\mathrm{HCl} \mathrm{pH} \mathrm{7.5,} \mathrm{5 \%}(w / v)$ glycerol and $1 \mathrm{mM}$ DTT at $4^{\circ} \mathrm{C} .100 \mathrm{nM}$ of DAC was incubated in $10 \mathrm{mM}$ Tris- $\mathrm{HCl}$ pH 7.5 with $100 \mu \mathrm{M}$ ATP, $10 \mathrm{mM}$ $\mathrm{MgCl}_{2}$ and $0.1 \%(w / v) B S A$ at $37^{\circ} \mathrm{C}$ for 30 min when the reactions were stopped and c-di-AMP extracted. Samples were incubated with or without 1 $\mathrm{ng} / \mu \mathrm{l}$ of $B$. subtilis CDNA and with or without the indicated concentration of $\mathrm{KCl}$. Data of three biological replicates are shown as the mean of two technical replicates per measurement point. Horizontal lines depict the mean of the three biological replicates. cDNA = chromosomal DNA.

DisA G334E mutation affects B. subtilis ability to adapt after salt stress - To investigated the effect of DisA and the G334E mutation on C-di-AMP concentrations and the capability of $B$. subtilis to adapt after a hyperosmotic stress in vivo, we constructed strains harboring DisA or the G334E 
mutant as sole DACs (BP172 and BP173, respectively). We grew the strains, referred to as DisA (wt) and DisA (G334E), in LB medium until exponential growth and shocked them by the addition of $\mathrm{LB}$ containing $\mathrm{NaCl}$ or $\mathrm{KCl}$ to a final concentration of $1.5 \mathrm{M}$ and compared the intracellular c-di-AMP concentration before the salt shock $\left(t_{0}\right)$, to $30 \mathrm{~min}\left(t_{1}\right)$ after it and 3 hours $\left(t_{2}\right)$ after it. We also compared the effects of salt shocked to non-salt shocked conditions (-osmolytes). As shown in Fig. 5.5, without osmolytes c-di-AMP levels do not change much from $t_{0}$ to $t_{1}$, but rise from $t_{1}$ to $t_{2}$, indicating higher c-di-AMP concentrations in later growth phases, which is consistent with previous reports (Corrigan et al., 2015).
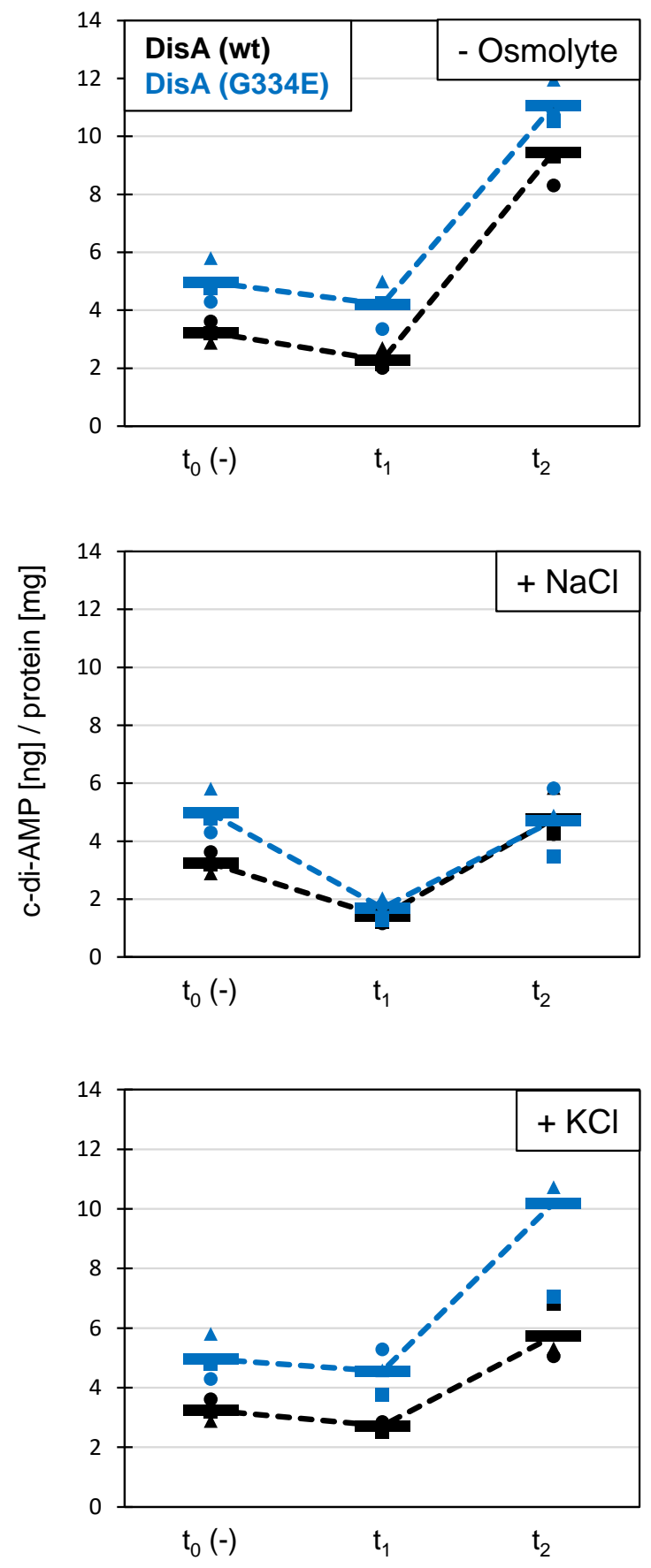

Fig. 5.5 DisA DNA-binding affects c-di-AMP concentration after osmotic stress in vivo. $B$. subtilis containing either the wild type DisA or the G334E mutant as sole DAC was grown in LB medium at $37^{\circ} \mathrm{C}$ until exponential growth. Samples for determination of the intracellular c-di-AMP concentration were taken $\left(t_{0}\right)$ and the cultures split to flasks with prewarmed LB medium with or without the indicated osmolytes and growth was resumed. The final concentrations of $\mathrm{NaCl}$ or $\mathrm{KCl}$ were $1.5 \mathrm{M}$. Samples for c-di-AMP concentration measurements were again taken after $30 \min \left(t_{1}\right)$ and 3 hours $\left(t_{2}\right)$. Data of three biological replicates are shown as the mean of two technical replicates per measurement point. Horizontal lines depict the mean of the three biological replicates.

Interestingly, in the G334E mutant, a higher c-di-AMP concentration is observed, with is in agreement with the higher observed concentration, if expressed in E. coli (Fig. S5.2). In NaCl-shocked cells, c-di-AMP concentrations rapidly decrease $\left(t_{0}\right.$ to $\left.t_{1}\right)$ and normalize afterwards $\left(t_{1}\right.$ to $\left.t_{2}\right)$, with 
no difference between the DisA wild type and DisA G334E expressing strain (Fig. 5.5). Interestingly, osmotic shock with $\mathrm{KCl}$ did not affect the initial c-di-AMP concentration ( $t_{0}$ to $\left.t_{1}\right)$, but was followed with an increase of intracellular c-di-AMP levels $\left(t_{1}\right.$ to $\left.t_{2}\right)$, with the mutant reaching about 2-fold higher c-di-AMP concentrations three hours after the salt shock (Fig. 5.5). This suggests that the cells are able to distinguish between the sources of the osmotic stress and adapt accordingly. Since we did not see a difference in the effects of $\mathrm{NaCl}$ and $\mathrm{KCl}$ in vitro, this effect is likely contributed to the PDEs, or the different - transport specific - abilities of the salts to accumulate intracellular, or a combination of both. It also demonstrates that DisA as sole cyclase is able to regenerate the c-di-AMP pool after osmotic stress and that the DNA-binding mutation of G334E can influence this ability. To assess whether the inhibitory effect of CDNA on DisA activity can be influenced by other potassium containing compounds, we took another look at the activity of DisA in vitro. We compared effects of $\mathrm{KCl}$ and potassium hydrogen phosphate (see experimental procedures) on the inhibitory effect on CDNA and surprisingly not only detected that inhibition by DNA was completely abolished in the presence of already $10 \mathrm{mM}$ potassium hydrogen phosphate (in terms of the potassium ions), but also activity of DisA seemed to be stimulated. In the presence of potassium hydrogen phosphate alone, the measured c-di-AMP concentration after $30 \mathrm{~min}$ was about 10 -fold higher, as in the untreated samples or the potassium chloride treated samples, indicating an effect of phosphate on DisA activity (Fig. S5.3).

Finally, we also tested whether a B. subtilis mutant containing DisA as sole DAC is able to recover after an osmotic stress and if the G334E mutant affects this ability. Similar to the c-di-AMP experiments, the strains BP172 (DisA (wt)) and BP173 (DisA (G334E)) were incubated in LB and shocked with the addition of $1.5 \mathrm{M}$ of the osmolytes $\mathrm{NaCl}, \mathrm{KCl}$ or sucrose and the growth was observed. As shown in Fig. 5.6, growth was inhibited after the osmotic shock for about two to three hours in all samples. In the case of sucrose, wt and G334E mutant displayed similar abilities to recover, with the mutant showing a slightly faster adaptation. Interestingly, in the case of the ionic osmolytes $\mathrm{NaCl}$ and $\mathrm{KCl}$, the mutant was able to adapt much faster, especially if the cells were shocked with $\mathrm{KCl}$, compared to the wild type. The wild type was also able to adapt, as all cultures had a similar $\mathrm{OD}_{600}$ after 20 hours (see Fig. S5.4). This indicates that DisA as sole cyclase is sufficient for B. subtilis to survive a hyperosmotic stress and that DNA-binding of DisA that seems to effect intracellular c-di-AMP concentrations is involved in the ability of DisA to fulfill this task.
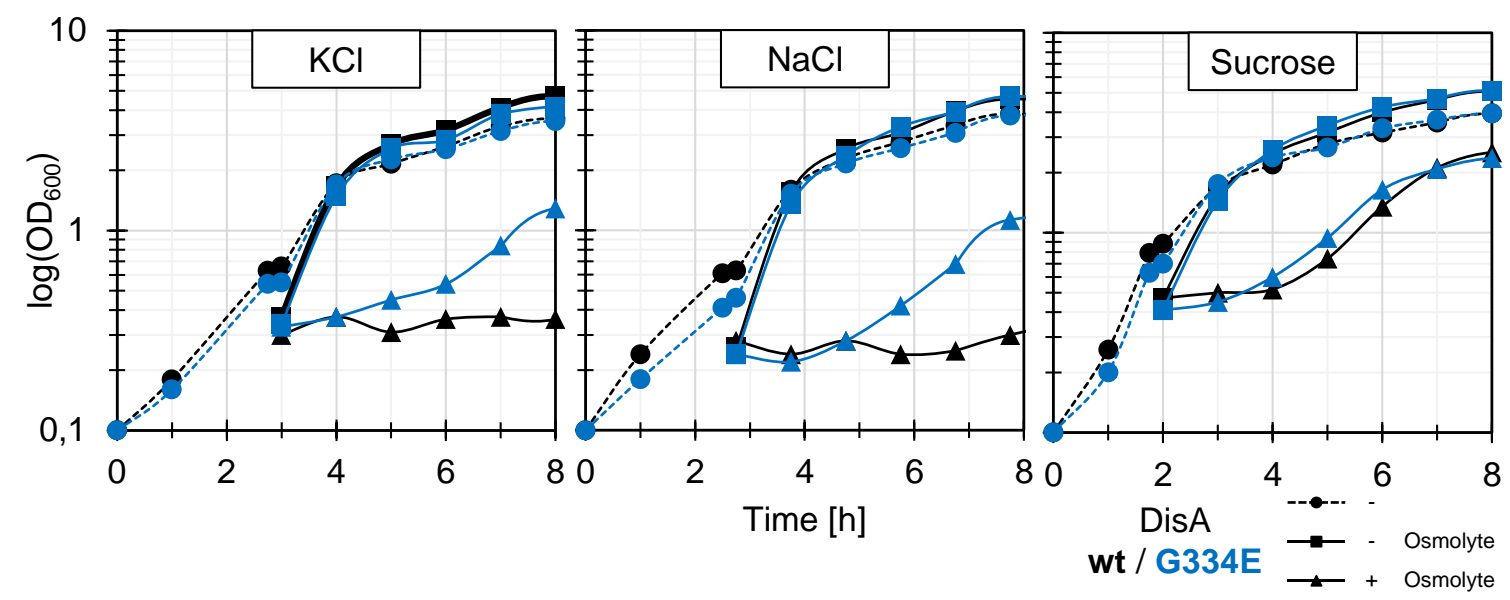

Fig. 5.6 The DisA G334E mutation affects resistance of $B$. subtilis to salt stress. B. subtilis containing either DisA (wt) or DisA (G334E) as sole DAC was grown in LB medium at $37^{\circ} \mathrm{C}$. Cultures were splitted in the exponential growth phase and either further incubated without change (-) or with after the addition of $1.5 \mathrm{M}$ of the indicated osmolytes (+ osmolytes) or an equivalent volume of LB without osmolytes (- osmolytes). Depicted are exemplary growth curves with hourly measurement of the $\mathrm{OD}_{600}$. 


\section{Discussion}

c-di-AMP has been shown to be essential due to its regulation of homeostasis of potassium and other osmolytes in a variety of bacteria (Devaux et al., 2018; Gundlach et al., 2017; Pham et al., 2018; Whiteley et al., 2015; Whiteley et al., 2017; Zeden et al., 2018). This discovery, the absence of DisA in many firmicutes and the apparent lack of DNA damage repair defects, which would be noticeable by increased accumulation of gene unspecific mutations and strain instability, lead us to challenge the current models of DisA regulation. The ability of DisA of being inhibited in DAC activity upon binding has been described simultaneously with the discovery of c-di-AMP and DisA as first DAC (Witte et al., 2008). Interestingly, Witte and colleagues did not see an effect of double or single stranded DNA on DisA activity, while in other studies also an effect of ssDNA has been observed (Gándara et al., 2017). Since we aimed to investigate the inhibitory effect of DNA on DisA activity in an osmoregulatory context, we chose to use chromosomal DNA as a DisA inhibitor, because it should in principle contain every possible conformation, from a bacterial population, including strand breaks and nicks from the purification and, furthermore, allows sequence independent observations. As shown in Fig. 5.1, DisA is indeed strongly inhibited by CDNA, in a concentration dependent matter and the effect is DisA-specific, as CdaS another DAC did not show an altered activity in the presence of cDNA. We wondered if dsDNA is also able to inhibit DisA activity in our monovalent cation-free assay setup and indeed, we saw an effect, although cDNA has a stronger inhibitory effect (Fig. S5.1). Interestingly, if we compare our experimental setup with previously published setups, it is likely that no inhibitory effects of dsDNA were observed in those studies due to $\mathrm{NaCl}$ concentrations of $50 \mathrm{mM}$ or above (Gándara et al., 2017; Witte et al., 2008). Interestingly, the study where $50 \mathrm{mM} \mathrm{NaCl}$ was in the reaction mixture observed an influence of ssDNA on DisA activity, which was not observed in the study using $100 \mathrm{mM}$, highlighting that assay ion concentrations can greatly influence behavior of DNA-binding proteins (Gándara et al., 2017; Witte et al., 2008). Indeed, it has also been reported that increase in extracellular potassium and the resulting increase of macromolecular crowding impacts the DNA-binding ability of the RNA polymerase (Cagliero \& Jin, 2013). Intriguingly, it has also been reported that a DisA mutant that interferes with dimerization of DisA molecules and therefore should not form octamers but tetramers via tetramerization of the HhH domains, showed salt sensitive dissociation of the tetramers with already $10 \mathrm{mM} \mathrm{NaCl}$ (Müller et al., 2015b). This further indicates that interaction of cations with DisA might impact its conformational arrangement and thereby may affect its DNA-binding properties.

Next, we assessed the influence of osmolytes on DisA activity in the presence of cDNA and did only observe increased DAC activity in the presence of sufficient amounts of the ionic osmolytes $\mathrm{NaCl}$ and $\mathrm{KCl}$, but not the non-ionic osmolyte sucrose in vitro (Fig. 5.2). Interestingly the ions $\mathrm{Mg}^{2+}$, $\mathrm{Ca}^{2+}, \mathrm{Na}^{+}$and $\mathrm{K}^{+}$are the main cationic ions associated with the negatively charged phosphate backbone of the DNA helix, the proposed binding site of DisA (Pasi et al., 2015; Witte et al., 2008). Since the DNA purification most likely does not result in salt-free DNA and the assay contains $10 \mathrm{mM}$ of $\mathrm{Mg}^{2+}$ ions, the effect of additional monovalent ions may be specific, but this has to be investigated in further studies. Remarkably, DisA activity was strongly increased in the presence of potassium hydrogen phosphate, which may indicate competition of the DNA phosphate backbone with free phosphate for the DisA DNA-binding domain or secondary, unknown effects (Fig. S5.3).

When assessing the in vitro role of the G334E mutation of DisA, which has been shown to reduce binding to branched nucleic acids and their inhibitory effect, we only observed a slight improve in potassium dependent release of DNA inhibition, but no change in DNA inhibition itself (Fig. 5.3 \& 
Fig. 5.4; Witte et al., 2008). Interestingly, the mutant showed the same activity as DisA wild type in vitro, but a higher activity in vivo if expressed in E. coli (Fig. S5.2). This indicates that activities of wild type and G334E mutant are not altered, but that the mutant is less-stronger inhibited as the wild type enzyme in vivo. Looking at the DisA octameric structure (Witte et al., 2008), it is obvious that DNA-binding domains of the two tetramers are facing opposite directions. We hypothesize that DisA interacts with dsDNA strands of the folded and condensed chromosome in vivo and that this is also the reason why strong effects of dsDNA have not been observed so far, since the used PCR products may be too short to allow the necessary folding for double-sided DisA interaction.

In vivo we demonstrated that DisA as sole DAC is able to take part in modulation of the intracellular c-di-AMP pool and adaptation to osmotic stress. Furthermore, the G334E mutant probably leads to a faster adaptation after an osmotic stress by contributing to a faster replenishment of the c-di-AMP pool, because it is less strongly inhibited by DNA (Fig. 5.5 \& Fig. 5.6). We therefore propose a model, in which DisA-DNA interaction is regulated by the osmotic conditions in dependency of cell size and ion concentration.

After a hyperosmotic shock, water will flow out of the cell, leading to shrinkage of the cellular volume, which results in a transiently higher concentration of DNA (Wood, 1999; Wood, 2011; Wood et al., 2001). DisA that has to compete with other DNA-binding proteins and affected by intracellular ion concentrations might now show a tighter binding to DNA or is more compressed between two DNA strands, resulting in inhibition of DAC activity. c-di-AMP is probably further degraded by the PDEs, allowing import of osmolytes that fist lead to an increase in internal potassium, followed by rehydration and finally import or de novo synthesis of compatible solutes. The first two might influence DisA binding to DNA, increasing c-di-AMP synthesis again to inhibit osmolyte importer again and prevent an accumulation that would be toxic for the cell (Gundlach et al., 2015; Gundlach et al., 2017). This might also explain the difference we saw in intracellular c-di-AMP levels, in dependency whether the osmolyte was $\mathrm{NaCl}$ or $\mathrm{KCl}$. For $\mathrm{NaCl}$, the above-mentioned model could be true, but for $\mathrm{KCl}$, c-di-AMP synthesis should not be lowered to much, since it would lead to a too fast and too high accumulation of intracellular potassium. The cells must therefore have evolved mechanisms to distinguish the source of the osmotic stress. While the CdaA cyclase may play a role as a general turgor sensor, DisA may act as a sensor for macromolecular crowding and intracellular ion concentration (see chapter 2). If the cells are exposed to a sudden upshift with potassium, the intracellular concentration would probably also rise immediately to a certain level due to active potassium import systems that are an important determinant for the intracellular turgor and thereby cell expansion (Whatmore \& Reed, 1990; Wood, 1999). This would result in a higher initial intracellular concentration of monovalent cations, opposed to $\mathrm{NaCl}$ and DisA would therefore presumably be less inhibited. The different effect of sucrose in vivo is probably due to $B$. subtilis ability to metabolize it and thereby quicker reduce its concentrations and the resulting osmotic pressure (Gay et al., 1983). For a hypoosmotic shock, c-di-AMP levels should be stable or rise to prevent further increase in intracellular osmolytes, with the simultaneously release of osmolytes to decrease the osmotic gradient. Here, DisA should stay active or even increase in activity, due to osmotic swelling and a subsequent decrease in DNA concentration (Wood, 1999) and it will be interesting to elucidate DisA function under those conditions.

On the basis of our findings, we think that it is compelling to postulate that the main regulator of DisA activity is not the DNA damage repair function, which our findings do not exclude, but that it is more likely a side function of DisA or that the related phenotypes are of secondary nature due to increased osmotic stress, as it has been proposed for other phenotypes (Commichau et al., 
2018). There are three main reasons for that. First, many bacteria produce c-di-AMP and its important role in osmoadaptation has been shown by different studies and many of the studied bacteria that are closely related to B. subtilis do not have a DisA homolog (Commichau et al., 2019; Devaux et al., 2018; Gundlach et al., 2017; Pham et al., 2018; Whiteley et al., 2015; Whiteley et al., 2017; Zeden et al., 2018). This makes it unlikely that DNA damage repair is a main function of DisA. Secondly, B. subtilis is viable without any obvious effects, like increased accumulation of mutations if only one DAC is present, independent of which or if any DAC is present at all, providing that the cells are grown under osmotically stable conditions, with a low potassium concentration (Gundlach et al., 2015; Gundlach et al., 2017; Mehne et al., 2013). Finally, it has been shown that this is also true for other bacteria. L. monocytogenes CdaA can be replaced by B. subtilis DisA, without any severe consequences, demonstrating that the role of DACs as synthesizer of c-di-AMP, as an osmolyte responsive second messenger, is interchangeable even between species, indicating a conserved role and source of regulation between DAC enzymes (Whiteley et al., 2017).

In the present study, we demonstrated inhibition of the enzyme DisA by cDNA on its DAC activity and the anti-inhibitory effect of ionic osmolytes on this effect in vitro. We furthermore could show that a G334E mutant is as inhibited by CDNA, but inhibition is easier relieved by potassium ions. Finally, we showed that $B$. subtilis with DisA as sole cyclase is able to adapt after an osmotic stress and that DisA is sufficient to modulate c-di-AMP concentrations accordingly. The G334E mutant thereby shows increased DAC activity in vivo and positively effects $B$. subtilis ability to adapt after a hyperosmotic shock by faster replenishing the intracellular c-di-AMP pool. We therefore show a DNA damage-independent role of regulation of DisA activity in the broader context of c-di-AMP as major regulator of osmohomeostasis in $B$. subtilis.

\section{Acknowledgements}

This work was supported by the grant CO 1139/2-1 from the Deutsche Forschungsgemeinschaft via the Priority Program SPP1879, the Fonds der Chemischen Industrie and the Max-Buchner-Forschungsstiftung (MBFSt-Kennziffer 3381) to FMC. 


\title{
6. The sRNA rli31 affects lysozyme resistance, motility and gene expression in Listeria monocytogenes
}

Johannes Gibhardt, Julian Schwanbeck, Samuel Hauf, Alexander Reder, Andrea Thürmer, Uwe Völker, Sven Halbedel and Fabian M. Commichau

Author contribution:

$J G$ and JS performed the experiments, JG analyzed the data, SH and AT and SH performed the RNASeq, AR and UV performed the protein quantifications, JG and FMC wrote the manuscript.

\begin{abstract}
Pathogenic bacteria have to overcome the host immune system and natural defensive barriers to successfully thrive inside the host. One important part of the innate immune system is the peptidoglycan (PG) hydrolyzing enzyme lysozyme. It hydrolyzes 1,4- $\beta$-linkages between $\mathrm{N}$-acetylmuramic acid (NAM) and $\mathrm{N}$-acetyl-D-glucosamine (NAG), leading to a weakening of the cell wall and subsequently lysis of the bacteria due to the high intracellular turgor pressure. Bacteria protect themselves against lysozyme by modifying their PG. Listeria monocytogenes produces the enzymes OatA, PgdA and PbpX that confer lysozyme resistance. The second messenger cyclic diadenosine monophosphate (c-di-AMP) has been linked to cell wall biogenesis and previous studies have linked the diadenylate cyclase (DAC) CdaA and its regulatory protein CdaR to increased resistance towards lysozyme. We evolved L. monocytogenes to become lysozyme resistant and identified suppressor mutations in the promoter region of the small RNA rli31. Interestingly, expression of $\mathrm{PbpX}$ and PgdA has been shown to be influenced by the small RNA rli31. We demonstrate that L. monocytogenes rapidly adapts towards lysozyme through acquisition of rli31 promoter mutations and show by RNA sequencing that the SRNA affects gene expression of genes involved in cell wall modifications, ion transport and motility and discuss possible cross-talk between rli31 and c-di-AMP signaling in L. monocytogenes.
\end{abstract}




\section{Introduction}

Pathogenic bacteria have to overcome several natural barriers to successfully infect their host, including physical barriers, like the epidermis or the acidic environment of the stomach, competition with organisms of the host's microbiota and evading detection by the immune system. The first response to a bacterial infection is facilitated by the humoral and cell-mediated response of the innate immune system, followed by responses of the adaptive immune system (Spiering, 2015). The glycoside hydrolase lysozyme is one of the first defenses bacteria encounter upon contact to the host. The enzyme class itself, however, is also important for bacteria to actively remodel the structure and composition of their cell wall. It hydrolyses the 1,4- $\beta$-linkage between the main PG glycan sugars NAM and NAG and has also pore forming properties. Through the weakening of the protective cell wall and the high turgor pressure, the bacteria will eventually lyse (Callewaert et al., 2010; Vollmer et al., 2008). Lysis and fragmentation of the PG furthermore leads to the release of components, like lipoteichoic acids that are subsequently detected by components of the innate immune system, leading to an inflammatory response (Ragland et al., 2017). Lysozyme is found ubiquitous in nature and can be found in higher animals, as well as, in invertebrates, highlighting its early evolutionary origin (Callewaert et al., 2010). In mammals, lysozyme is found in high concentrations on the skin, in the blood, liver, urine, milk, tear fluid, on mucosal surfaces and it can reach high concentrations of about $1 \mathrm{mg} / \mathrm{ml}$ (Callewaert et al., 2010; Ragland et al., 2017). Bacteria have therefore evolved mechanisms to counteract lysozyme. Counteracting a bactericidal compound is in general achieved by several different mechanism: degradation of the compound, secretion if it acts intracellular, biofilm formation as a diffusion barrier or modulating the target of the compound either through regulatory processes or genetically by acquisition of mutations or horizontal gene transfer, as it is often the case for the development of antibiotic resistances (Munita \& Arias, 2016). Interestingly, recent studies have shown that bacteria may have adapt to lysozyme in a way that it can be beneficial for surviving antibiotic treatment. It has been shown that bacteria can adapt to a cell wall-less life style, called L-forms, under osmotically stabilizing conditions, as they are for example present in a host cell. Treatment of lysozyme promote transition to the L-form lifestyle and renders the bacteria thereby immune against cell wall acting antibiotics, like $\beta$-lactam antibiotics, which could be a clinically relevant new mode of persistence against antibiotic treatment (Burke, 2018; Kawai et al., 2018). In case of lysozyme, the modification of its target, the PG is the prevalent mechanism, but there are also bacteria, like Pseudomonas aeruginosa and Escherichia coli that synthesize lysozyme inhibitors (Clarke et al., 2010; Monchois et al., 2001; Ragland et al., 2017). Bacteria synthesize for example the enzymes PgdA, OatA and PbpX that N-deacetylate NAG, O-acetylate NAM or carboxylase the PG, respectively, and therefore decrease the binding affinity of lysozyme to it, rendering the cells immune (Burke et al., 2014; Ragland et al., 2017). Synthesis of these enzymes has to be regulated in order for the bacteria to adapt to their environment. In case of L. monocytogenes, the transcriptional activator PrfA is the major regulator of virulence determinants. Environmental signals, such as the temperature, and molecules like glutathione influence PrfA amount and activity, resulting in a fine-tuned expression of virulence related genes (de las Heras, et al., 2011; Lobel et al., 2015; Reniere et al., 2015; Scortti et al., 2007). In a similar manner L. monocytogenes has to detect the presence of lysozyme and other cell wall-acting compounds and adapt its gene expression accordingly. For L. monocytogenes the regulation of gene expression or translation via cis-acting RNA elements, like the temperature-depended prfA riboswitch that controls expression of PrfA or via trans-acting RNA elements, like small RNAs (sRNAs), play an important role. They can modulate translation by binding to mRNA, protein activity by RNA-protein interaction (Mellin \& Cossart, 2012; Sesto et al., 2014 Thorsing et al., 2018). One of these sRNAs is rli31. It has been identified in 
a transposon mutagenesis study by Burke and colleagues to regulate expression of the PG modulating enzymes PgdA and PbpX (Burke et al., 2014). The mode of action of rli31 however remained elusive, as no direct interaction of it has been identified, so far. Interestingly, rli31 interacts with the protein SpoVG and also with the mRNA encoding it, but the consequence of this interaction is unknown. SpoVG has been proposed to be an RNA-binding posttranscriptional regulator (Burke et al., 2016). Another impact factor on the bacterial cell wall is the nucleotide second messenger c-di-AMP. It is synthesized by DACs and degraded by specific phosphodiesterases (PDEs) and it has been shown to be essential in firmicutes (Commichau et al., 2015). c-di-AMP has been implicated in regulation of various cellular processes, ranging from DNA damage repair, over the regulation of lifestyle changes and the modification of the cell wall, to the regulation of osmotic homeostasis, by regulation of ion and compatible solute transport processes (Corrigan et al., 2011; Gundlach et al., 2016; Gundlach et al., 2017; Oppenheimer-Shaanan et al., 2011; Pham et al., 2018; Whiteley et al., 2015; Whiteley et al., 2017; Witte et al., 2008; Witte et al., 2013; Zeden et al., 2018). It has been suggested that many cell wall related phenotypes of c-di-AMP, such as the decreased resistance of DAC mutants to cell wall-acting antibiotics, may just be indirect effects due to c-di-AMPs role as major regulator of osmohomeostasis (Commichau et al., 2018). It is however established that there is some cross-talk between c-di-AMP signaling and cell wall biogenesis. The gene encoding the only DAC in many pathogenic firmicutes $c d a A$ is co-transcribed with the gene cdaR, encoding a regulator of CdaA activity and this operon is genetically conserved with the $\mathrm{g} / \mathrm{mM}$ gene, encoding the phosphoglucosamine mutase GlmM (Gundlach et al., 2015; Mehne et al., 2013; Rismondo et al., 2016). Interestingly GImM is both, an essential enzyme that converts glucosamine-6-phosphate to glucosamine-1-phosphate, one of the earliest precursor molecules for NAM and NAG biosynthesis and a repressor of CdaA activity (Tosi et al., 2019; Zhu et al., 2016; see chapter 2). As shown in chapter 4, a cdaA mutant shows altered gene expression and protein biosynthesis of pathways involved in cell wall metabolism, which further suggest a direct connection between c-di-AMP signaling and cell wall metabolism. Furthermore, we previously identified that a cdaR deletion mutant is increased resistant towards lysozyme and another study identified, among others, mutations in the $c d a A$ gene upon evolving Streptococcus suis towards lysozyme resistance (Rismondo et al., 2016; Wichgers Schreur et al., 2012). We therefore set out to evolve lysozyme resistant $L$. monocytogenes, to elucidate the connection between the osmoregulatory function of c-di-AMP signaling and its interconnections to the cell wall metabolism. Strikingly, we found, as previously studies reported, a connection between the sRNA rli31 and lysozyme resistance and could further elucidate the role of the sRNA on regulation of gene expression and highlight possible interconnections to the c-di-AMP signaling network.

\section{Experimental Procedures}

Bacterial strains and growth conditions - L. monocytogenes 10403S, EGD-e and EGD-e mutants were cultivated in $\mathrm{BHI}$ medium (Sigma-Aldrich) at $37^{\circ} \mathrm{C}$ and $220 \mathrm{rpm}$ if not specified otherwise. E. coli was grown in LB medium at $37^{\circ} \mathrm{C}$ and $220 \mathrm{rpm}$ (strains see Tab.6.1). For agar plates, medium was supplemented with $15 \mathrm{~g} / \mathrm{l}$ Bacto Agar (Difco). Antibiotics and medium supplements were used with the following concentrations, if indicated: erythromycin $(5 \mu \mathrm{g} / \mathrm{ml}), \mathrm{X}-\mathrm{Gal}(100 \mu \mathrm{g} / \mathrm{ml} ; 5-$ bromo-4-chloro-3-indolyl- $\beta$-D-galactopyranoside; Sigma-Aldrich). Lysozyme from chicken egg white (15000 units/mg; Serva) and penicillin G (Serva) were used in the indicated concentrations.

DNA manipulation - DNA amplification via PCR and transformation of $E$. coli was performed using standard procedures (Sambrook et al., 1989). DNA fragments were purified using the PCR 
purification kit (Qiagen) and plasmid DNA was extracted using the NucleoSpin Plasmid Kit (Macherey and Nagel). Commercially available restriction enzymes, T4 DNA ligase and DNA polymerases were used as recommended by the manufacturers. DNA sequences were determined by the dideoxy chain termination method (Microsynth, Göttingen, Germany). Chromosomal DNA of L. monocytogenes was isolated using the NucleoSpin Microbial DNA Kit (Macherey and Nagel). Oligonucleotides were purchased from Sigma-Aldrich (Germany).

Tab. 6.1 Strains, Plasmids and Oligonucleotides

\begin{tabular}{|c|c|c|c|}
\hline \multicolumn{4}{|l|}{ Strains } \\
\hline Name & Genotype & Description/Construction & Reference \\
\hline \multicolumn{4}{|l|}{ E. coli } \\
\hline XL1-Blue & $\begin{array}{l}\text { recA1 endA1 gyrA96 thi-1 hsdR17 supE44 relA1 } \\
\left.\text { lac }\left[\mathrm{F}^{\prime} \text { proAB lac/q } Z \Delta \mathrm{M} 15 \mathrm{Tn} 10 \text { (Tet }{ }^{r}\right)\right]\end{array}$ & Cloning & Stratagene \\
\hline \multicolumn{4}{|c|}{ L. monocytogenes } \\
\hline $10403 \mathrm{~S}$ & Wild type & Serotype $1 / 2$ a strain & Laboratory collection \\
\hline EGD-e & Wild type & Serotype $1 / 2$ a strain & Laboratory collection \\
\hline BPL10 & $\Delta f$ liil:: $\mathrm{P}_{\text {alf4}}-\mathrm{RBS}$ hly $-m g f p(\mathrm{~A} 206 \mathrm{~K})$ & $\begin{array}{l}\text { flil and reverse oriented } \\
\text { replacement by mgfp }\end{array}$ & Chapter 4 \\
\hline BPL23 & EGD-e $\Delta p g p H$ & $\begin{array}{l}\text { Chromosomal deletion of } \\
\text { pgpH }\end{array}$ & Chapter 4 \\
\hline BPL24 & EGD-e $\Delta p d e A$ & $\begin{array}{l}\text { Chromosomal deletion of } \\
\text { pdeA }\end{array}$ & Chapter 4 \\
\hline LMJR45 & EGD-e $\Delta c d a R$ & $\begin{array}{l}\text { Chromosomal deletion of } \\
\text { cdaR }\end{array}$ & Rismondo et al., 2016 \\
\hline BPL25 & $\begin{array}{l}\text { EGD-e } P_{\text {rli31 }} \quad\left(G_{597,748} A\right) \quad C_{1,224,119} T \quad \text { (Imo1199, } \\
\text { silent) }\end{array}$ & $\begin{array}{l}\text { Suppressor } 2-1 \quad(2 \mathrm{mg} / \mathrm{ml} \\
\text { lysozyme) }\end{array}$ & This work \\
\hline BPL26 & $\begin{array}{l}\text { EGD-e Prli31 }\left(T_{597,758} T T\right) \quad G_{2,348,663} A \quad \text { (Imo2622, } \\
\text { H232Y) }\end{array}$ & $\begin{array}{l}\text { Suppressor } 2-11(2 \mathrm{mg} / \mathrm{ml} \\
\text { lysozyme) }\end{array}$ & This work \\
\hline BPL27 & EGD-e $P_{r l i 31}\left(T_{597,758} T T\right)$ & $\begin{array}{l}\text { Suppressor } 4-I(4 \mathrm{mg} / \mathrm{ml} \\
\text { lysozyme) }\end{array}$ & This work \\
\hline BPL28 & EGD-e $P_{r l i 31}\left(G_{597,759} A\right)$ & $\begin{array}{l}\text { Suppressor } 4-1 \mathrm{l}(4 \mathrm{mg} / \mathrm{ml} \\
\text { lysozyme) }\end{array}$ & This work \\
\hline BPL34 & EGD-e $\Delta r l i 31$ & pBP340 $\rightarrow \rightarrow$ EGD-e & This work \\
\hline \multicolumn{4}{|l|}{ Plasmids } \\
\hline Name & Insert/Features & & Reference \\
\hline pMAD & bla ermC bgaB & & Arnaud et al., 2004 \\
\hline pBP340 & pMAD- $\Delta$ rli31 & & This work \\
\hline \multicolumn{4}{|c|}{ Oligonucleotides } \\
\hline Name & \multicolumn{2}{|c|}{$\begin{array}{l}\text { Restriction sites are underlined, complementary regions are in bold, se- } \\
\text { quences } 5^{\prime} \rightarrow 3^{\prime}\end{array}$} & Purpose \\
\hline JSL08 & \multicolumn{2}{|c|}{$\begin{array}{l}\text { AAACTCGAGCACATTTTTTCGCGTGCTGGAAAGCATAATTATGTAGAAATAGA- } \\
\text { GATGCTCTGAA }\end{array}$} & $\begin{array}{l}\text { Fwd. rli31 downstream } \\
\text { region (Xhol) }\end{array}$ \\
\hline JSL09 & \multicolumn{2}{|c|}{ TTTGGATCCCGTATTCAAAGTGAGCACGTTCATTTCTATCAATC } & $\begin{array}{l}\text { Rev. rli31 downstream } \\
\text { region (BamHI) }\end{array}$ \\
\hline JSL10 & \multicolumn{2}{|l|}{ TTTGAATTCCGATAGATACCTTGGCTCTCGGCTTTG } & $\begin{array}{l}\text { Fwd. rli31 upstream re- } \\
\text { gion (EcoRI) }\end{array}$ \\
\hline JSL11 & \multicolumn{2}{|c|}{$\begin{array}{l}\text { GCTTTCCAGCACGCGAAAAATGTGCTCGAGTTTTCTCTATGGGA- } \\
\text { TAAGTATATCTTACATTACTTTTATG }\end{array}$} & $\begin{array}{l}\text { Rev. rli31 upstream re- } \\
\text { gion }(X h o l)\end{array}$ \\
\hline
\end{tabular}

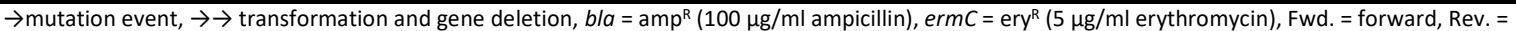
reverse 
Plasmid construction - For the chromosomal deletion of the rli31 gene, pBP340 was constructed. The up- and downstream regions of the 144nt large sRNA rli31 (Imos23; genome position 597,806$597,949)$, while sparring out the rli31 ORF and leaving promoter and downstream regions intact (deletion from 597,822-597,941), were amplified using oligonucleotide pairs JSL10/JSL11 and JSL08/JSL09, respectively. The resulting PCR products were fused by SOE PCR using oligonucleotides JSL10 and JSL09, digested with ECoRI and BamHI and ligated to pMAD (Arnaud et al., 2004; Horton et al., 1990), which was digested using the same enzymes (plasmids and oligonucleotides are listed in Tab. 6.1).

L. monocytogenes strain construction - For the chromosomal deletion of the rli31 gene, strain BPL34 was constructed as follows. Electrocompetent cells were prepared as described by Monk and colleagues and electroporation performed as previously described (Monk et al., 2008; chapter 2). The EGD-e wt was electroporated with plasmid pBP340 (pMAD- $\Delta r l i 31$ ) and plated on BHI medium with erythromycin and X-Gal at $30^{\circ} \mathrm{C}$ for up to $72 \mathrm{~h}$. Single, blue colonies were streaked on the same medium and incubated for up to $72 \mathrm{~h}$ at $42^{\circ} \mathrm{C}$ to force integration into the rli31 locus. Several blue colonies were used to inoculate $5 \mathrm{ml}$ of $\mathrm{BHI}$ without antibiotics at $30^{\circ} \mathrm{C}$ for $4 \mathrm{~h}$, temperature was shifted to $42^{\circ} \mathrm{C}$ for $6 \mathrm{~h}$, after which serial dilutions were plated on BHI medium with $\mathrm{X}$-Gal and incubated at $37^{\circ} \mathrm{C}$ for up to $72 \mathrm{~h}$. Erythromycin-sensitive, X-Gal negative bacteria were subjected to colony PCR as described elsewhere (Dussurget et al., 2002). rli31 deletion was confirmed by Sanger sequencing and the strain designated BPL34.

Evolution experiment, isolation of lysozyme resistant suppressor mutants and WGS - L. monocytogenes EGD-e was grown in $\mathrm{BHI}$ medium from different single colonies overnight at $37^{\circ} \mathrm{C}$ and $220 \mathrm{rpm}$. Pre-cultures were used to inoculate $20 \mathrm{ml} \mathrm{BHI}$ medium to an $\mathrm{OD}_{600}$ of 0.05 with or without 2 or $4 \mathrm{mg} / \mathrm{ml}$ of lysozyme. Bacteria were incubated for 18 hours at $37^{\circ} \mathrm{C}$ and $220 \mathrm{rpm}$, the $\mathrm{OD}_{600}$ measured and the cells re-inoculated into fresh medium with the same lysozyme concentration. This was repeated for 4 passages until improved growth in the presence of lysozyme was visible and cultures with lysozyme reached similar $\mathrm{OD}_{600}$ after 18 hours as the wild type without lysozyme. Emerging suppressor mutants were re-streaked twice on $\mathrm{BHI}$ agar plates and two independent colonies of each concentration and the EGD-e wild type strain were subjected to WGS. WG illumina sequencing was performed by the G2L Göttingen, the resulting reads analyzed using the Geneious software (Geneious Prime 2019.0.4 (https://www.geneious.com)) and potential mutations re-sequenced by Sanger Sequencing (Microsynth, Göttingen). The resulting suppressor mutants were designated BPL25, BPL26, BPL27 and BPL28, which are clone I and II, respectively of the evolution with $2 \mathrm{mg} / \mathrm{ml}$ lysozyme (BPL25 \& BPL26) and with $4 \mathrm{mg} / \mathrm{ml}$ (BPL27 \& BPL28). BPL27 was further investigated and is here also referred to as $\mathrm{P}_{\text {rli31 }}{ }^{*}$ (concept based on Barrick \& Lenski, 2013).

Motility assay - The L. monocytogenes wild type strain, the flil mutant BPL10 (see chapter 4), rli31 mutant BPL34 and the $\mathrm{P}_{\text {rli31 }}{ }^{*}$ mutant BPL27 were used to assess the impact of rli31 on the motility. Strains were cultivated in $5 \mathrm{ml} \mathrm{BHI}$ overnight at $37^{\circ} \mathrm{C}$ and $220 \mathrm{rpm}$. The pre-cultures were used to inoculate $5 \mathrm{ml}$ of $\mathrm{BHI}$ medium to an $\mathrm{OD}_{600}$ of 0.05 and the cells were incubated at $37^{\circ} \mathrm{C}$ and $220 \mathrm{rpm}$. Cells were harvested and resuspended in fresh $\mathrm{BHI}$ medium after reaching an $\mathrm{OD}_{600}$ of about 0.5 and $1 \mu \mathrm{l}$ of $\mathrm{OD}_{600} 1.0$ cell suspensions were used to stab inoculate $\mathrm{BHI}$ soft agar plates $\left(0.3 \%(w / v)\right.$ agar). Plates were incubated at $25^{\circ} \mathrm{C}$ or $37^{\circ} \mathrm{C}$, as indicated and imaged every $24 \mathrm{~h}$.

Lysozyme autolysis assay - To assay if the isolated suppressor mutants were indeed more lysozyme resistant and the possible effect of c-di-AMP on lysozyme resistance, the L. monocytogenes wild type strains EGD-e and 10403S, as well as the EGD-e based mutants of $p g p H, p d e A, c d a R$ (BPL23, BPL24, LMJR45) and the evolved lysozyme resistant suppressor mutants (BPL25-BPL28) 
were analyzed for their ability to withstand lysozyme. Bacteria were inoculated from overnight cultures in $10 \mathrm{ml} \mathrm{BHI}$ medium to an $\mathrm{OD}_{600}$ of 0.05 and grown until they reached an $\mathrm{OD}_{600}$ of 0.8 \pm 0.1 . Cells were harvested by centrifugation $\left(3300 \mathrm{~g}, 4^{\circ} \mathrm{C}, 10 \mathrm{~min}\right)$. The cells were washed in $50 \mathrm{mM}$ Tris- $\mathrm{HCl}, \mathrm{pH} 8$ and finally adjusted in the same buffer to an $\mathrm{OD}_{600}$ of $1.2 .10 \mu \mathrm{g} / \mathrm{ml}$ of lysozyme, $25 \mu \mathrm{g} / \mathrm{ml}$ penicillin $\mathrm{G}$ or nothing was added and decline of $\mathrm{OD}_{600}$ observed using $100 \mu \mathrm{lsam}$ ples in 96-well plates (Microtest Plate $96-$ Well,F, Sarstedt) at $37^{\circ} \mathrm{C}$ and $237 \mathrm{cpm}(4 \mathrm{~mm})$ using an Epoch2 multiwell plate reader, equipped with the Gen5 software (02.09.2001; BioTek Instruments). The $\mathrm{OD}_{600}$ was normalized to the $\mathrm{OD}_{600}$ at time point 0 (\% of initial $\mathrm{OD}_{600}$ ) and mean values of four biological replicates were used to assess the different strains (modified from Rismondo et al., 2016).

Growth of L. monocytogenes $\Delta$ rli31 and $P_{\text {riiz1 }}{ }^{*}$ mutants for transcriptomic and proteomic analysis - To analyze the changes in the transcriptome and proteome of $L$. monocytogenes between the deletion and promoter mutant of rli31, strains BPL34 and BPL27 were cultivated and processed as follows. Single colonies were propagated from $\mathrm{BHI}$ agar plates to $10 \mathrm{ml} \mathrm{BHI}$ medium overnight at $37^{\circ} \mathrm{C}$ and $220 \mathrm{rpm}$. Pre-cultures were used to inoculate $100 \mathrm{ml} \mathrm{BHI}$ medium to an $\mathrm{OD}_{600}$ of 0.05 and incubated at $37^{\circ} \mathrm{C}$ and $220 \mathrm{rpm}$. For the analysis of the proteome $40 \mathrm{OD}_{600}$ units of cells were harvested by rapid cooling in liquid nitrogen to slow down cellular processes and subsequent centrifugation after reaching an $\mathrm{OD}_{600}$ of about 0.5 at $3300 \mathrm{~g}$ and $4^{\circ} \mathrm{C}$ for $10 \mathrm{~min}$. Cell pellets were washed twice in ZAP ( $50 \mathrm{mM}$ Tris $\mathrm{pH} 7.5$ and $200 \mathrm{mM} \mathrm{NaCl}$ ), with centrifugation steps in between. Resulting pellets were frozen in liquid nitrogen and stored at $-80^{\circ} \mathrm{C}$ until further processing. Growth for the analysis of the transcriptome was performed similar. When the cultures reached $\mathrm{OD}_{600}$ of $0.5 \pm 0.05,25 \mathrm{ml}$ were collected and quenched by adding of $25 \mathrm{ml}$ ice cold killing buffer (20 mM Tris- $\mathrm{HCl} \mathrm{pH} \mathrm{7.5,} 5 \mathrm{mM} \mathrm{MgCl}_{2}, 20 \mathrm{mM} \mathrm{NaN}_{3}$ ). After 5 min incubation on ice, cells were harvested by centrifugation. mRNA extraction, library preparation and RNA sequencing where subsequently performed as described previously in detail (Hauf et al., 2019).

$m R N A$ isolation and RNA sequencing - Further processing was performed as previously described (see chapter 4).

Protein isolation and proteome analysis - Further processing was performed as previously described (see chapter 4).

Data analysis - The in gene expression or protein biosynthesis affected genes and proteins were later on analyzed using the Geneious software (Geneious Prime 2019.0.4 (https://www.geneious.com)) and the online resources ListiWiki (http://listiwiki.uni-goettingen.de) and SubtiWiki (http://subtiwiki.uni-goettingen.de), the Listeriomics website (https://listeriomics.pasteur.fr) and the annotation databases RAST and COG (Aziz et al., 2008; Bécavin et al., 2017; Tatusov et al., 2000; Zhu et al., 2018).

\section{Results}

L. monocytogenes rapidly adapts to lysozyme - To elucidate the ability of L. monocytogenes to adapt to lysozyme, the EGD-e wild type strain was evolved in the presence of this muralytic enzyme. The bacteria were cultivated in BHI without or with 2 or $4 \mathrm{mg} / \mathrm{ml}$ lysozyme and passaged every 18 hours. As depicted in Fig. 6.1 , the $\mathrm{OD}_{600}$ after 18 hours of growth increased in the cultures treated with lysozyme already in the second passage and reached wild type levels in passage three and four. This evolution experiment demonstrates on the one hand that even in the first two 
passage L. monocytogenes is able to withstand quite high lysozyme concentrations and moreover that the bacteria can rapidly adapt to lysozyme stress and after grow like the wild type even with lysozyme concentrations four times higher as have been reported in human liquids (Ragland et al., 2017). From the last passage putative suppressor mutants were isolated and subjected to WGS.

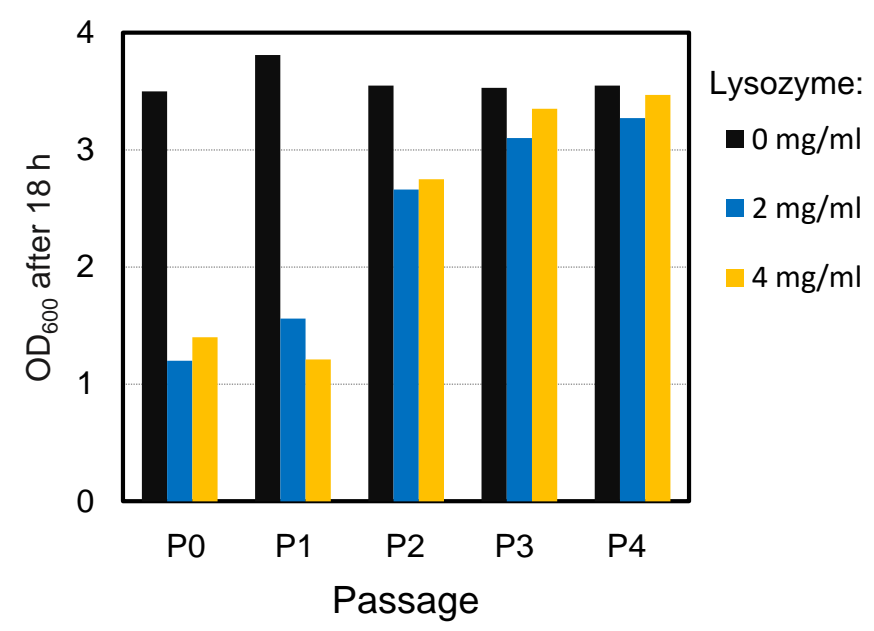

Fig. 6.1 L. monocytogenes rapidly adapts to lysozyme. The $L$. monocytogenes EGD-e wild type strain was inoculated in $\mathrm{BHI}$ medium and grown at $37^{\circ} \mathrm{C}$. Pre-cultures were used to inoculate $\mathrm{BHI}$ fresh medium to an $\mathrm{OD}_{600}$ of 0.05 without or with the indicated concentration of lysozyme. Every $18 \mathrm{~h}$ the $\mathrm{OD}_{600}$ was measured and fresh medium inoculated to an $\mathrm{OD}_{600}$ of 0.05 . Passage four was subsequently used to isolate lysozyme resistant suppressor mutants

L. monocytogenes EGD-e adapts to lysozyme by acquisition of mutations in the promoter of the sRNA rli31 - WGS of the suppressor mutants revealed that all four isolates acquired mutations upstream of the gene Imos23 that encodes the sRNA rli31 (Fig. 6.2, A). Further analysis revealed that the mutations affect a region that shows all characteristics of a prokaryotic promoter region. As depicted in Fig. 6.2, B, suppressor mutants 2-I and 2-II, which were evolved in the presence of $2 \mathrm{mg} / \mathrm{ml}$ lysozyme have acquired mutations in the spacing region between the -35 and -10 regions (guanine to adenine exchange and a thymine insertion, respectively). Suppressor mutants 4-I has acquired the same mutation as mutant 2-II and suppressor mutant $4-I I$ has a mutation in the -10 region, leading to a guanine to adenine exchange. Interestingly, the mutation of isolate 4-II seems to be a natural occurring variant in L. monocytogenes, as it is the wild type allele of the $10403 \mathrm{~S} \mathrm{wt}$ strain. The guanine to adenine mutation in the spacing region probably leads to a lower melting temperature and the thymine insertion mutations to an improved spacing between the -35 and 10 region. The mutation in the -10 region results in an optimal TATAAT sequence. We therefore assumed that all mutations increased the expression of the adjacent sRNA rli31. The expression of the promoter and rli31 probably also influence the expression of the downstream located gene Imo0559, encoding a putative magnesium or cobalt transporter. Predictions of transcriptional start sites and terminators suggest that Imo0559 does not have its own promoter region and that, although transcriptional terminators are predicted between rli31 and Imo0559, they are in part co-transcribed (Listeriomics website, Bécavin et al., 2017; Dar et al., 2016; Wurtzel et al., 2012). It has to be noted that suppressor mutants $2-I$ and $2-I I$ had second site mutations. Mutant 2-I harbored a silent mutation in the gene Imo1199, encoding CbiH a precorrin-3B C17-methyltransferase involved in cobalamin biosynthesis and mutant 2-II had a mutation in the gene Imo2622, leading to a histidine to tyrosine exchange of amino acids 232 in the encoded RpIN, a ribosomal protein (LSU ribosomal protein L14p/L23e). Since all four isolates evolved similar mutations in the promoter of rli31, we assumed that the mutations in isolates 2-I and 2-II were second site mutations and not primary reason for the improved growth in the presence of lysozyme. The mutation could however be due to increased expression of rli31 and therefore be potential targets of the sRNA. 
A

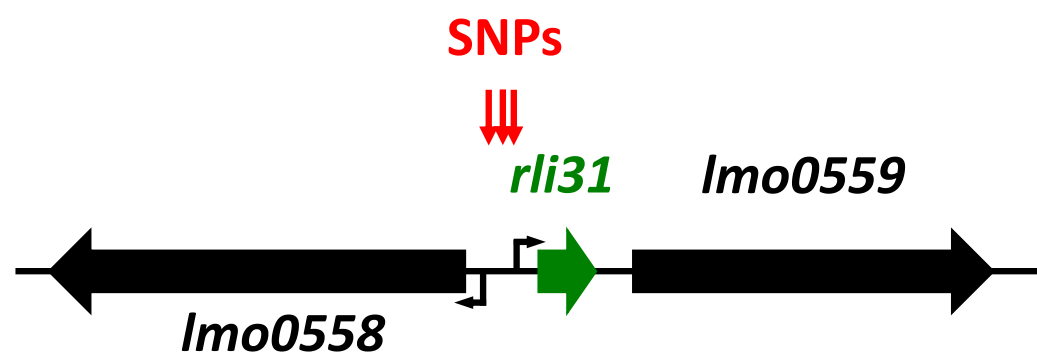

B

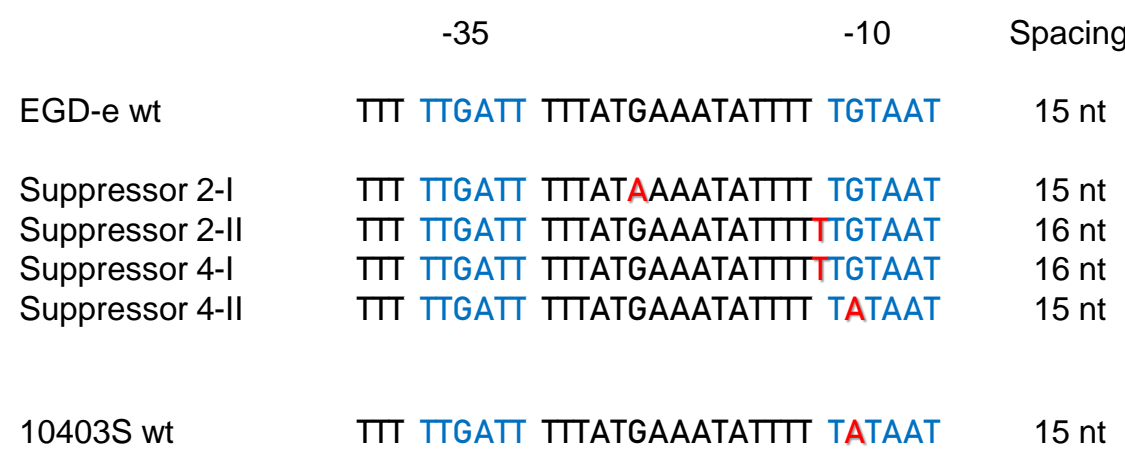

Fig. 6.2 Genome position of the identified SNPs upstream of the sRNA rli31 and comparison of the promoter mutations. WGS revealed SNPS in the same region in all four lysozyme resistant isolates of L. monocytogenes EGD-e. (A) Illustration of the rli31 operon. The SNPs are located between the two genes of unknown function Imo0558 and Imo559. The sRNA rli31 is localized between these two genes. (B) Comparison of the identified mutations. The SNPs were identified as mutations of the rli31 promoter. Suppressor 2-I harbors a guanine to adenine exchange in the spacing region between the -35 and -10 region, suppressor 2-II and 4-I harbor an additional thymine in the spacing region and suppressor 4-II a has evolved a guanine to adenine change in the -10 region. The later mutation is also present as a natural variation in the L. monocytogenes wild type strain 10403s. Suppressor $2 / 4=$ isolated from BHI with 2 or $4 \mathrm{mg} / \mathrm{ml} \mathrm{lyso-}$ zyme, respectively, WGS = whole genome sequencing, SNP = single nucleotide polymorphism, $\mathrm{nt}=$ nucleotide

Evaluation of the lysozyme resistance of the suppressor mutants - To assess the if the isolated suppressor mutants are indeed increased resistant against lysozyme, we performed autolysis assays. The wild type strain, the evolved mutants and the $10403 \mathrm{~S}$ wt strain, with the naturally -10 promoter variant found in suppressor 4-II, where assayed together with mutants affecting the c-di-AMP metabolism to investigate a possible link between c-di-AMP signaling, lysozyme resistance and rli31. We evaluated the previously as increased lysozyme resistant identified $c d a R$ deletion mutant and deletion mutants of the $p d e A$ and $p g p H$ genes, encoding the two c-di-AMP specific PDEs in L. monocytogenes. It was hypothesized previously that the increased intracellular concentration of c-di-AMP in the cdaR mutant is responsible for the increased lysozyme resistance and we assumed the PDEs should behave similar if the resistance is due to c-di-AMP and not caused by the CdaR protein itself (Rismondo et al., 2016). Bacteria were grown in BHI to the late exponential phase and cells of an $\mathrm{OD}_{600}$ of 1.2 were resuspended in $50 \mathrm{mM}$ Tris- $\mathrm{HCl}$, pH 8. Subsequently lysozyme $(10 \mu \mathrm{g} / \mathrm{ml})$, penicillin $\mathrm{G}(25 \mu \mathrm{g} / \mathrm{ml})$ or nothing was added and decline of $\mathrm{OD}_{600}$ at $37^{\circ} \mathrm{C}$ was recorded. Without addition of lysozyme or penicillin $\mathrm{G}$, the $\mathrm{OD}_{600}$ remained quite stable over the measured time period and only no extreme lysis was visible, demonstrating that the different strains do not show lysozyme or penicillin G independent autolysis (Fig. S6.1). With the addition of penicillin $\mathrm{G}$, the $\mathrm{OD}_{600}$ decreased to about $80 \%$ of the initial $\mathrm{OD}_{600}$ and state stable at this range over the measured time, without difference between the strains, demonstrating that resistance against $\beta$-lactam antibiotics is not influenced by rli31 or the c-di-AMP mutants under the tested conditions (Fig. S6.1). 
As shown in Fig. 6.3, the $\mathrm{OD}_{600}$ of the EGD-e wild type strain rapidly decreased in the presence of lysozyme, indicating lysis. In contrast to that, 10403S wild type strain lysed with a much slower rate, indicating a higher resistance towards lysozyme. The $c d a R$ mutant showed as previously reported an increased resistance towards lysozyme (Rismondo et al., 2016). The pdeA mutant showed an intermediate phenotype between wt and $c d a R$, while the $p g p H$ mutant showed the same rate of lysis as the wt.

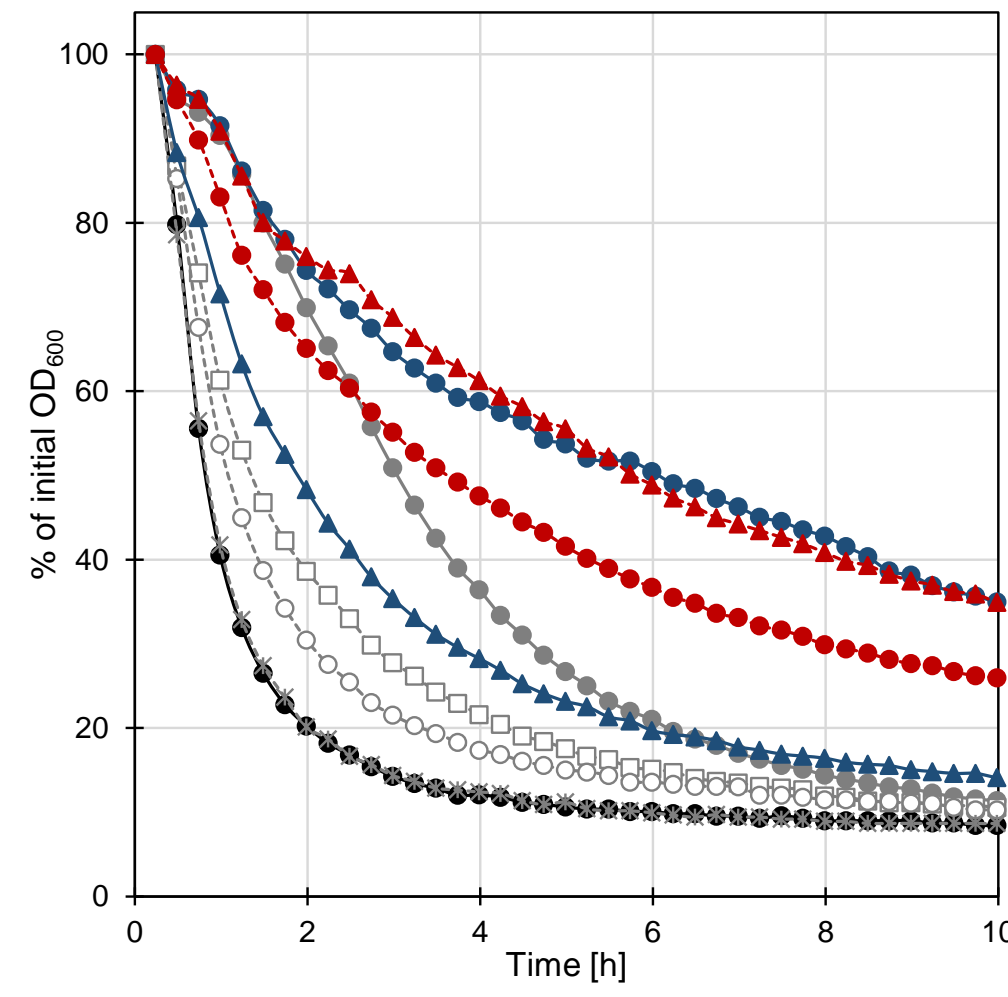

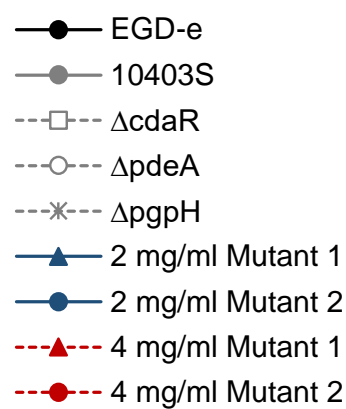

Fig. 6.3 The $P_{\text {rli31 }}$ suppressor mutants are resistant against lysozyme. The depicted strains were grown in $\mathrm{BHI}$ at $37^{\circ} \mathrm{C}$ until they reached an $\mathrm{OD}_{600}$ between of about 0.8. Cells were washed in $50 \mathrm{mM}$ Tris $\mathrm{pH} 8$ and the suspensions adjusted to an $\mathrm{OD}_{600}$ of $1.2 .10 \mu \mathrm{g} / \mathrm{ml}$ lysozyme was added and the cell suspensions incubated at $37^{\circ} \mathrm{C}$ and the decline in $\mathrm{OD}_{600}$ measured in 15 min intervals. Depicted are the mean values of four biological replicates.

Comparing the ability of the evolved suppressor strains to withstand lysozyme, mutant 2-I ( $G \rightarrow A$ in the spacing region) showed the lowest increase of lysozyme resistance, between the cdaR mutant and the $10403 \mathrm{~S}$ wt. Mutant $4-2$, which has the same -10 region sequence as the $10403 \mathrm{~S}$ strain, showed a great increase in resistance compared to the EGD-e strain and also an increased resistance compared to the $10403 \mathrm{~S}$ strain, suggesting that downstream effects, like targets of the sRNA may have evolved apart in these two strains. Finally, mutant 2-II and 4-I, having the improved spacing between the -35 and -10 promoter regions, showed the highest increase in resistance, suggesting highest expression of rli31 in those two isolates. To conclude all four isolates indeed showed increased resistance towards lysozyme. Next, we wanted to identify the regulatory network of the sRNA. We therefore constructed a $\triangle$ rli31 mutant (BPL34) and compared gene expression and protein biosynthesis using RNA sequencing and whole proteome mass spectrometry, respectively. We also investigated strain BPL27 (suppressor mutant 4-I), showing the highest lysozyme resistance and no second site mutations and the wild type EGD-e strain as a reference. The mutant 4-I will be referred to as $\mathrm{P}_{\text {rli31 }}$ * from here on.

rli31 affects expression of peptidoglycan remodeling and motility genes in L. monocytogenes - The analysis of changes in gene transcription between the $\Delta r l i 31$ mutant and the wild type showed no significant change in the gene expression except for a 40-fold decrease in Imo0559 expression, the downstream gene of ri31. This suggests that deletion of rli31 effects expression of the gene. It remains to be elucidated if this effect is a regulatory one or an unwanted side effect. In the $\mathrm{P}_{\text {rliz1 }}{ }^{*}$ mutant, no significant changes were detected that showed a at least 2 -fold change in expression 
compared to the wild type. The less than 2 -fold expression changes are nevertheless shown in Tab. S6.1. We therefore compared expression levels between the rli31 deletion mutant and $\mathrm{P}_{\text {rli31 }}{ }^{*}$, that were significant and showed a greater than 2-fold change. Unfortunately, only the genes Imo0559 and Imo1336, encoding a 5-formyltetrahydrofolate cyclo-ligase, matched those criteria. We therefore lowered the significance cut-off to $p<0.05$ and the cut-off for fold-change to 1.5 to detect possible weak influence of $r$ li31 that may explain the how rli31 leads to lysozyme resistance. The results are shown in Tab. 6.2 and further information, including the p-values and even less sure deregulated genes can be found in Tab. S6.1.

Tab. 6.2 Genes differently regulated in $\Delta$ rli31 compared to $P_{\text {ril31 }}{ }^{*}$

\begin{tabular}{|c|c|c|c|c|}
\hline Locus tag & Operon & Name & RAST info & Fold change \\
\hline Imo05591 & & & Magnesium and cobalt transport protein CorA & -70.92 \\
\hline Imo2805 & 508 & & hypothetical protein & -2.12 \\
\hline Imo0540 & & pbpX & penicillin-binding protein, putative & -1.84 \\
\hline Imo2150 & & & FIG00774336: hypothetical protein & -1.71 \\
\hline Imo0620 & & & FIG00774170: hypothetical protein & -1.60 \\
\hline$\underline{\operatorname{Imo} 1494}$ & 246 & mtn & $\begin{array}{l}\text { 5'-methylthioadenosine nucleosidase / } \\
\text { S-adenosylhomocysteine nucleosidase }\end{array}$ & 1.49 \\
\hline Imo0689 & 111 & cheV & Chemotaxis protein $\mathrm{CheV}$ & 1.51 \\
\hline Imo0688 & 111 & gmaR & glycosyl transferase, group 2 family protein & 1.52 \\
\hline Imo2077 & 378 & ydic & $\begin{array}{l}\text { Inactive homolog of metal-dependent proteases, putative } \\
\text { molecular chaperone }\end{array}$ & 1.54 \\
\hline Imo1047 & 168 & moaA & Molybdenum cofactor biosynthesis protein MoaA & 1.54 \\
\hline Imo1912 & 343 & dgcB & GGDEF domain protein, DGC & 1.55 \\
\hline Imo2758 & & guaB & Inosine-5'-monophosphate dehydrogenase & 1.58 \\
\hline Imo1903 & & & bacteriocin transport accessory protein & 1.59 \\
\hline Imo2667 & 477 & & PTS system, galactitol-specific IIA component & 1.60 \\
\hline Imo0715 & 112 & fliH & Flagellar assembly protein FliH & 1.60 \\
\hline Imo1911 & 343 & $\operatorname{dgc} A$ & GGDEF domain protein, DGC & 1.62 \\
\hline Imo0934 & 144 & yhbA & Epoxyqueuosine (oQ) reductase QueG & 1.62 \\
\hline Imo0710 & 112 & flgB & Flagellar basal-body rod protein FlgB & 1.67 \\
\hline Imo1913 & 343 & ydaJ & Lmo1913 protein & 1.69 \\
\hline $\operatorname{Imo} 2575$ & 462 & CzCD & Cobalt-zinc-cadmium resistance protein CzcD & 1.72 \\
\hline Imo1045 & 168 & & Molybdenum cofactor biosynthesis protein MoaD & 1.74 \\
\hline Imo0585 & & & extracellular protein & 1.76 \\
\hline Imo2586 & 465 & yrhE & Formate dehydrogenase related protein & 1.77 \\
\hline Imo0718 & 112 & & FIG00774152: hypothetical protein & 1.79 \\
\hline Imo0716 & 112 & flil & Flagellum-specific ATP synthase Flil & 1.81 \\
\hline Imo0901 & & gmuC & PTS system, cellobiose-specific IIC component & 1.84 \\
\hline Imo1034 & 166 & glpK & Unknown pentose kinase TM0952 & 1.89 \\
\hline Imo1336 & & yqgN & 5-formyltetrahydrofolate cyclo-ligase & 2.37 \\
\hline Imo0755 & 119 & ypmR & FIG006988: Lipase/Acylhydrolase with GDSL-like motif & 2.44 \\
\hline Imo0324 & & & FIG00774236: hypothetical protein & 2.75 \\
\hline Imo0699 & 112 & flim & Flagellar motor switch protein FliM & 2.77 \\
\hline Imo0349 & 58 & & FIG00774379: hypothetical protein & 3.60 \\
\hline Imo0685 & 111 & motA & Flagellar motor rotation protein MotA & 4.44 \\
\hline
\end{tabular}

RAST= Rapid Annotation using Subsystem Technology (database), grey names=homologs of $B$. subtilis 169 (ListiWiki), gene names in underlined $=$ signifi-

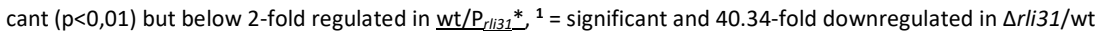


Only four genes, besides Imo0559 showed a slight downregulation in the $\Delta$ rli31 strain compared to the $\mathrm{P}_{\text {rli31 }}{ }^{*}$ suppressor mutant. Three hypothetical proteins of unknown function and Imo0540 encoding the peptidoglycan carboxylase $\mathrm{PbpX}$, that has been shown to be rli31 regulated by an unknown mechanism (Burke et al., 2014). The upregulated genes were fascinatingly mainly associated with motility, like fliM, flil, motA, flgB, fliH, cheV and interestingly the gene encoding the MogR anti-repressor GmaR (Imo0688) that is necessary for expression of flagellar genes at temperatures below the physiological $37^{\circ} \mathrm{C}$ (Gueriri et al., 2008; Gründling et al., 2004). Besides the motility genes, the genes Imo0755 (encoding a putative phospholipase) and Imo2575 (encoding a putative cation exporter) could be of interest in the lysozyme phenotype by their putative actions on the cell envelope and ion transport (and therefore osmoregulatory effects). Interestingly with Imo1911 and Imo1912 two genes are upregulated that encode the c-di-GMP diguanylate cyclases DgcA and DgcB, which might indicate an involvement of c-di-GMP signaling (Chen et al., 2014). Finally, the genes Imo1336 that encodes a protein involved in tetrahydrofolate metabolism, in which cobalamin plays a role were upregulated, as well. In suppressor mutant 2-I a silent mutation in Imo1199 was found, encoding $\mathrm{CbiH}$ that is involved in cobalamin biosynthesis and as seen in Tab. S6.1 the Imo1148 gene, encoding the cobalamin synthase might be differently expressed, in dependency of rli31, suggesting a possible regulatory context between them. We also analyzed possible changes of rli31 on the protein expression, but unfortunately no statistically significant differences could be measured. We nevertheless included the results in the supplementary Tab. S6.1 and annotated for the genes Imo0540, encoding PbpX and Imo0559, which lies downstream of ri31 that in all Prli31* samples peptides were found but no peptides were found in the rli31 mutant, suggesting strong downregulation in this strain. Interestingly, as seen in Tab. S6.1, we also detect, even though not significant a nearly 2-fold change in Imo0415 expression, which encodes the second known target of rli31 (Burke et al., 2014).

rli31 affects motility in L. monocytogenes EGD-e - To assess whether the potential changes in gene expression also lead to phenotypes, we assessed the influence of rli31 on the motility of L. monocytogenes. As expected, no motility was observed at $37^{\circ} \mathrm{C}$ due to the temperature-dependent repression of motility and also the flil mutant showed no motility at $25^{\circ} \mathrm{C}$ (Gueriri et al., 2008; Gründling et al., 2004; Halbedel et al., 2012; see Fig. 6.4). The rli31 deletion mutant showed a similar migration as the wild type and only the $\mathrm{P}_{r l i 31} *$ mutant showed a slight decrease in motility.

$25^{\circ} \mathrm{C}$

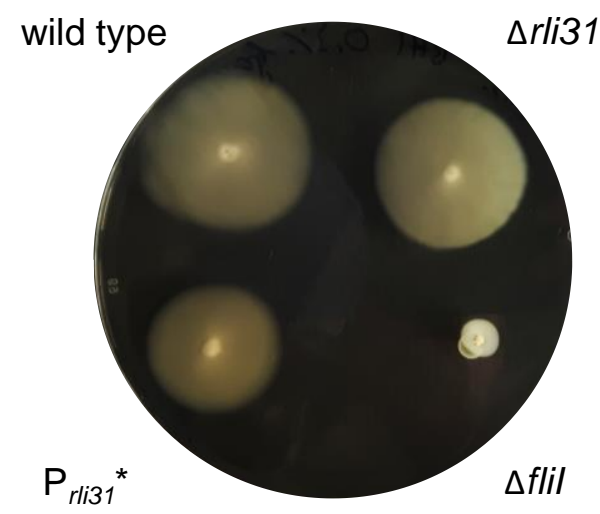

$37^{\circ} \mathrm{C}$

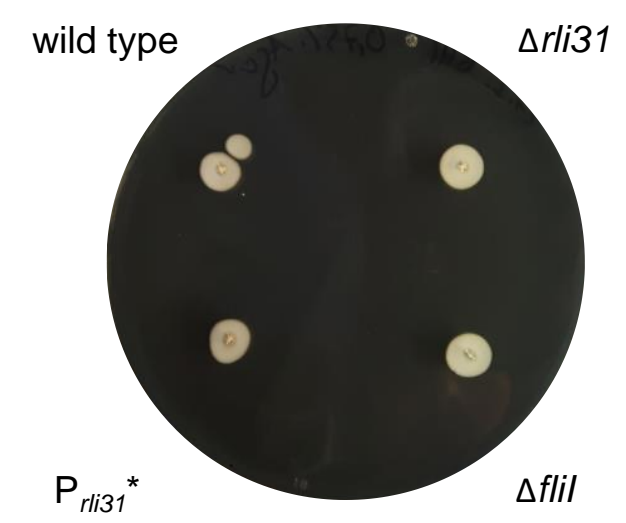

Fig. 6.4 rli31 affects motility of $L$. monocytogenes. The wild type strain, the mutants $\Delta r$ li31, the suppressor mutant 4-I $\left(\mathrm{P}_{\text {rli31 }}{ }^{*}\right)$ and a flil::mgfp mutant were investigated for their ability to spread on $\mathrm{BHI}$ agar plates. Bacteria were grown in $\mathrm{BHI}$ medium and $1 \mu \mathrm{l}$ of cells with an $\mathrm{OD}_{600}$ of 1 were stab inoculated in $\mathrm{BHI}$ plates containing $0.3 \%(w / v)$ agar. Plates were incubated at $25^{\circ} \mathrm{C}$ and $37^{\circ} \mathrm{C}$ for 3 days. 


\section{Discussion}

In the present study, we demonstrated that $L$. monocytogenes EGD-e rapidly adapts lysozyme resistance by acquisition of mutations in the promoter of the sRNA rli31 (Fig. 6.1 \& Fig. 6.2). Interestingly, in a study by Burke and colleagues they found rli31 as a regulator of lysozyme resistance the other way around. They screened for the 104035 wild type using a transposon library for lysozyme sensitive mutants and identified rli31, pgdA, pbpX, degU, wall, dltB, dltD, the vir operon, prsA2, Imo2473 and Imo2768 as insertion loci that lead to lysozyme sensitivity (Burke et al., 2014). Of these only rli31, pgdA, $p b p X$ and $\operatorname{deg} U$ showed lysozyme sensitivity, but no sensitivity against, cationic antimicrobial peptides or cell wall-acting antibiotics, suggesting that those four are involved in a lysozyme specific phenotype. Of those we only see an effect of rli31 on expression of $p g d A$ and $p b p X$, confirming the findings of Burke and colleagues that rli31 affects their expression, even though no direct interaction of rli31 was observed (Burke et al., 2014; Tab. S6.1). So far, only the known binding targets of rli31 are the protein and mRNA of SpoVG in vitro, which they could show to be a global RNA-binding posttranscriptional regulator (Burke et al.,2016). Interestingly, deletion of spovG rescued the lysozyme sensitivity and also the in vivo virulence attenuation of the rli31mutant and a spoVG mutant was non-motile. Remarkably, RNase J1 mutants arose, which could restore swarming motility. These observations lead to the interesting hypothesis that rli31 is indeed involved in regulation of motility. (i) A degU mutant affects lysozyme resistance and DegU is important for regulation of motility gene expression (Burke et al., 2014; Dons et al., 1992; Gueriri et al., 2008; Mauder et al., 2008; Peel et al., 1988). (ii) rli31 binds the spoVG mRNA and the SpoVG protein in vitro and a deletion of spoVG leads to motility defects that require suppressor mutations in an RNA degrading enzyme (RNase J1) to regain swarming motility (Burke et al., 2016). (iii) Motility genes are differently expressed in a rli31 mutant, compared to an evolved $\mathrm{P}_{\text {rli31 }}$ * lysozyme resistant suppressor (Tab. S6.1). (iv) the $\mathrm{P}_{\text {rli31 }}{ }^{*}$ mutant shows decreased swarming motility (Fig. 6.4). It is unknown how rli31 might influence motility gene expression, but the transcriptomic data suggests a few possibilities. On the one hand, expression of c-di-GMP synthesizing enzymes seems to be altered. In E. coli, c-di-GMP is a major regulator of motility, while in B. subtilis no effect of c-di-GMP has been shown, but motility is instead regulated by the interaction of the proteins SIrR and Sinl with SinR and motility genes are upregulated in a high c-di-AMP gdpP PDE mutant (Gundlach et al., 2016; Mukherjee \& Kearns; Vlamakis et al., 2013). The role of c-di-GMP in L. monocytogenes is not well studied, but increased levels lead to higher synthesis of an exopolysaccharide that inhibits motility, suggesting that the situations in B. subtilis and L. monocytogenes are different (Chen et al., 2014; Gao et al., 2013; Köseoğlu et al., 2015). Increased levels of c-di-GMP may also influence gene expression of motility genes or of genes required for lysozyme resistance, but this has yet to be elucidated. The role of c-di-AMP in motility of $B$. subtilis and L. monocytogenes is another interesting aspect. While high c-di-AMP concentrations seem to increase expression of motility genes in $B$. subtilis, low c-di-AMP levels seem to lead to the same phenotype in L. monocytogenes, suggesting fundamental differences in regulation of motility gene expression (Gundlach et al., 2016; chapter 4). In B. subtilis motility is mainly regulated by interaction of Sinl or SlrR with SinR that integrates environmental signals and regulate expression of biofilm and motility genes in a mutual exclusive order. B. subtilis has furthermore the DegSU two component system (Mukherjee \& Kearns; Vlamakis et al., 2013). In contrast to this, L. monocytogenes motility is mainly regulated by the ambient temperature. At physiological temperatures, motility is repressed by MogR, whose mRNA is at the same time an antisense RNA to flilNQ mRNA. If the temperature sinks to $30^{\circ} \mathrm{C}$ and below, the thermosensor protein $\mathrm{GmaR}$, which as a moonlighting enzyme, also modifies the flagellin, becomes stable and less prone to degradation, due to conformational changes and binds MogR. Binding prevents GmaR degradation and allows 
transcription of the motility genes by DegU. DegU is an orphan response regulator, without sensory histidine kinase, in L. monocytogenes and regulation of it is still not fully known. An increase in temperature eventually leads to dissociation of the MogR-GmaR complex, degradation of GmaR and inhibition of motility gene expression, because with MogR present, the effect of DegU is not strong enough (Dons et al., 1992; Gueriri et al., 2008; Kamp \& Higgins, 2008; Kamp \& Higgins, 2011; Mauder et al., 2008; Peel et al., 1988; Shen et al., 2006). Interestingly, expression of gmaR is 1.5-fold up-regulated in the $\Delta$ rli31 mutant compared to $\mathrm{P}_{\text {rli31 }} *$ (Tab. S6.1). This indicated gmaR repression in a rli31-dependent manner, which would lead to a decrease in motility gene expression in L. monocytogenes with an increased expression of rli31 and therefore lysozyme resistance (Fig. 6.4). It has to be noted that these data were gathered at $37^{\circ} \mathrm{C}$ when gmaR expression is usually repressed. It will be interesting to see the effect of rli31 at lower temperatures and to see if gmaR expression is even more elevated. In the context of the pathogenic lifestyle of L. monocytogenes, the downregulation of motility genes upon contact with lysozyme and probable overexpression of rli31 as a consequence would make sense, since flagellin is a potent trigger of the innate immune system (Ragland et al., 2017). As described in chapter 4, c-di-AMP affects motility gene expression, as well. The common grounds of lysozyme resistance of $c d a R, p d e A$ and $P_{r l i 31}$ mutants and altered motility in a c-di-AMP and rli31 dependent matter, led us to reinvestigate the differences in gene expression that have been described previously (chapter 4). Surprisingly, we found overexpression of Imo0559 and Imo0560, the two genes directly downstream of rli31 (see Fig. 6.5)

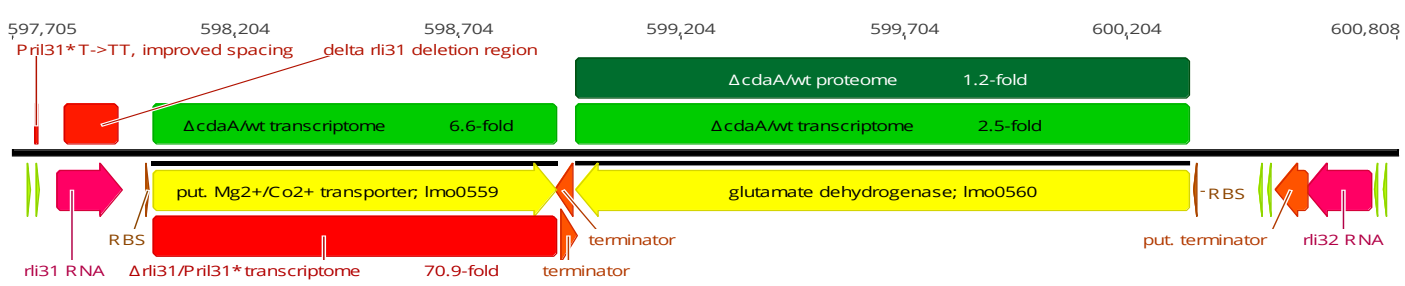

Fig. 6.5 c-di-AMP signaling and rli31. Depicted is the locus of the sRNA rli31. It lies upstream of the Imo0559 gene encoding a putative magnesium or cobalt transporter. Downstream of 1 mo0559 lies the reverse orientated Imo0560 gene encoding the glutamate dehydrogenase of L. monocytogenes, followed by the sRNA rli32. The sites of mutation in $\mathrm{P}_{\text {rli31 }}{ }^{*}$ and the deletion region in $\Delta$ rli31 are depicted, as well as transcriptional changes in gene expression of $\Delta$ rli31 vs. the $\mathrm{P}_{\text {rli31 }} *$ (Imo0559,70.9-fold downregulated). Furthermore, changes in gene expression of $\Delta c d a A$ vs. the wild type (see chapter 4; light green, Imo0559 6.6- and Imo0560 2.5-fold upregulated) and protein abundancy (dark green 1.2-fold higher abundancy of Lmo0560) are shown. $-10 /-35=$ promoter regions, RBS = ribosomal binding site, vs. = versus.

While expression of $I m o 0560$, encoding the glutamate dehydrogenase of L. monocytogenes, may be independent of rli31, it may be influenced by rli32 that lies in front of the gene (although both have their own promoters and terminators). Most interesting is that Imo0559 is upregulated in a cdaA deletion mutant. As described in the results part, expression of Imo0559 probably depends on rli31 expression, due to the lack of an own transcriptional start site. Nucleotides smaller than $200 \mathrm{nt}$ were not included in the performed RNA sequencing, meaning the $144 \mathrm{nt}$ sRNA could not be detected. Assuming that $r l i 31$ is upregulated in a cdaA mutant, it should negatively affect gmaR expression and thereby inhibit motility, suggesting that either an unknown factor is at play or that c-di-AMP and rli31 lead to similar phenotypes utilizing different pathways. A tempting hypothesis is that c-di-AMP can modulate rli31 binding to its targets in addition of regulating its expression, there is however no experimental evidence supporting this hypothesis, at the moment. Nevertheless, an interesting connection and possible cross-regulation of sRNA regulation and c-di-AMP signaling that is worth further analysis in the future. We therefore propose the following model for 
a possible regulation of motility and cell wall remodeling genes by rli31, which is depicted in Fig. 6.6.

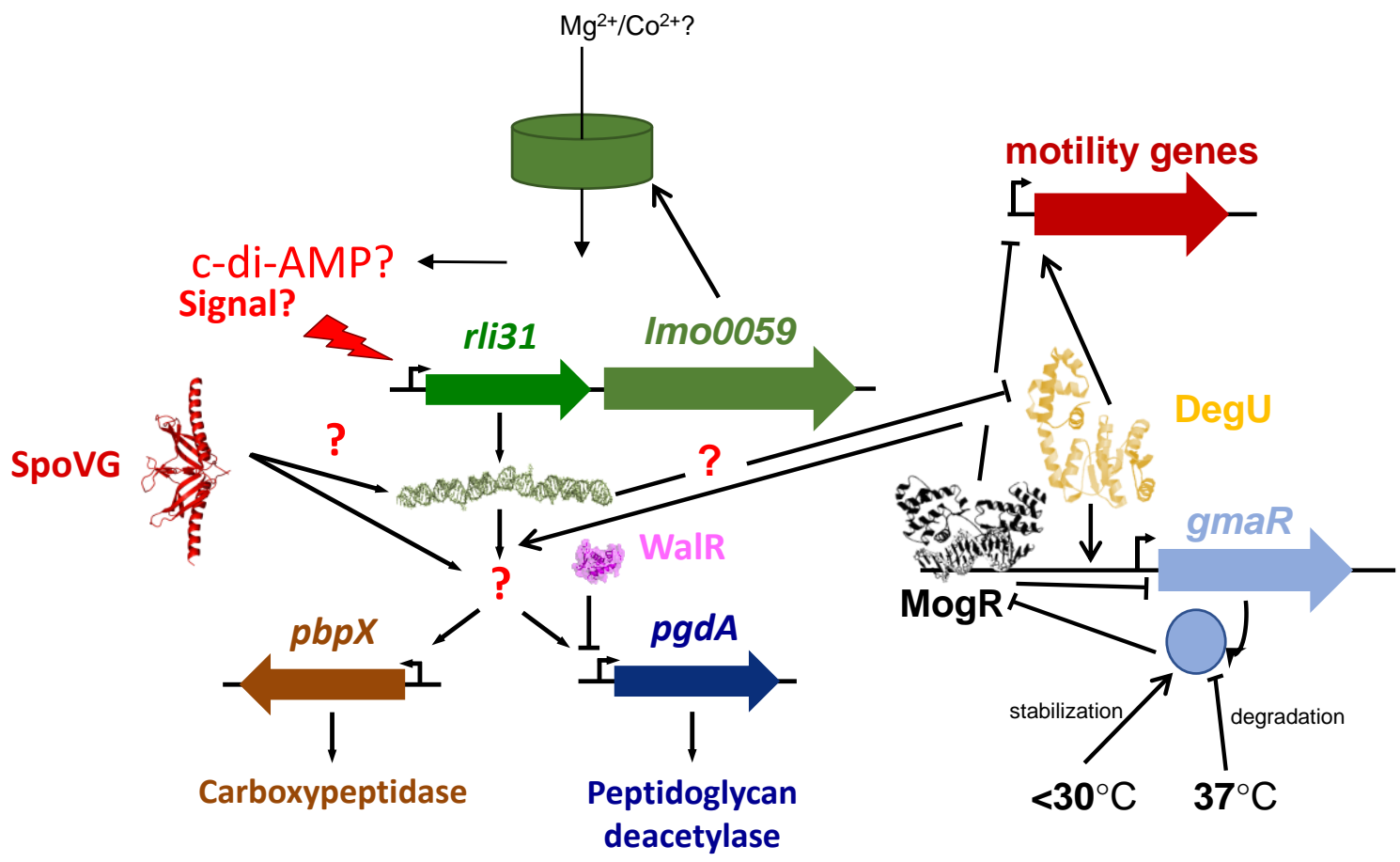

Fig. 6.6 Model depicting the role of rli31 in peptidoglycan remodeling. The sRNA rli31 has been shown to affect the expression of the carboxypeptidase PbpX and the peptidoglycan deacetylase PgdA that is also regulated by the WalR response regulator. The RNA chaperon SpoVG has further been identified as interacting with the sRNA with unknown outcome. So far, neither the signal, nor the direct target are known. Our findings have shown that rli31 is furthermore involved in regulation of motility genes and also the expression of the downstream of rli31 located gene, Imo0059, encoding a putative magnesium or cobalt ion transporter may be influenced by rli31 expression. Interestingly, rli31, leads to higher expression of gmaR that encoded the anti-repressor of MogR that might lead to altered expression of motility genes. DegU, which is also involved in gmaR expression and in lysozyme resistance may be the most promising target for rli31. It is also possible that c-di-AMP or a c-di-AMP-derived signal affects expression of rli31, as the downstream gene Imo0559, which shares the TSS with rli31 is overexpressed in a cdaA mutant.

c-di-AMP or a c-di-AMP-derived signal that indicates cell wall or osmotic stress leads to upregulation of rli31-Imo0559 expression. Putative increased import of magnesium or cobalt ions could influence either DAC activity, as divalent ions are cofactors for c-di-AMP synthesis, with cobalt for L. monocytogenes DAC in vitro (Rosenberg et al., 2015). Or act as osmolytes and thereby alter DAC activity (see chapter 2). Increased expression leads to modulation of DegU in a way that it no longer stimulates gmaR and motility gene expression, but switches to expression of PG remodeling enzymes. Interestingly, Burke et al., found no impact of rli31 on expression or translation of $\operatorname{deg} U$ and vice versa, they did however not investigate RNA-protein interaction, because rli31 deletion or overexpression did not have a motility phenotype in the used 10403s wt strain (Burke et al., 2014). Fascinatingly, in the EGD-e wt strain only the $P_{r l i 31}{ }^{*}$ had a slight influence on motility (Fig. 6.4), suggesting a difference between $10403 S$ and EGD-e in regulation of motility and indeed it has been shown that MogR of $10403 \mathrm{~S}$ does not complement the more stringent temperature phenotype of EGD-e MogR, highlighting possible differences in regulation of motility gene expression in the more pathogenic $10403 \mathrm{~S}$ and the more attenuated EGD-e strain (Glaser et al., 2001; Gründling et al., 2004). Another explanation is that over expression of rli31 only lacks a phenotype in 10403S, because it already has a stronger promoter (Fig. 6.1, B) and further overexpression of rli31 may not increase its effect on motility, but only on lysozyme resistance, if the first one is only a side effect by increased recruitment of DegU to the genes $p g d A$ and $p b p X$ (direct regulation by 
DegU has not been investigated yet and is therefore hypothetical). rli31 may, therefore, act as a modulator of the orphan response regulator DegU, controlling its targets and this in cross-talk with the c-di-AMP signaling network as a possible signal input for cell envelope stress.

Acknowledgements

This work was supported by the grant CO 1139/2-1 from the Deutsche Forschungsgemeinschaft via the Priority Program SPP1879, the Fonds der Chemischen Industrie and the Max-Buchner-Forschungsstiftung (MBFSt-Kennziffer 3381) to FMC. 
Cyclic di-AMP and osmoregulation in Listeria monocytogenes 


\section{Discussion}

\section{Regulation of diadenylate cyclase activity}

In this study the synthesis of the nucleotide second messenger cyclic di-AMP (c-di-AMP), its function on regulating potassium homeostasis in Listeria monocytogenes and its global effect on gene expression are investigated.

c-di-AMP is synthesized by enzymes containing a diadenylate cyclase (DAC) domain and in the recent years it has become increasingly implicated in regulation of osmotic homeostasis in several bacteria (Devaux et al., 2018; Gundlach et al., 2017; Pham et al., 2018; Whiteley et al., 2015; Whiteley et al., 2017; Zeden et al., 2018). In the time before discovering that both, essentiality and major function of c-di-AMP are related to osmotic homeostasis, the regulation of cell wall homeostasis was the most prominent hypothesis for the main function of c-di-AMP signaling (Commichau et al., 2015; Corrigan et al., 2011; Dengler et al., 2013; Luo \& Helmann, 2012; Rismondo et al., 2016; Witte et al., 2013). Regulation of cell wall homeostasis may not be the main function or reason of its essentiality and many cell wall-related phenotypes might be secondary effects, as a result of an altered osmoregulation (Commichau et al., 2018). Nevertheless, osmotic homeostasis and cell wall remodeling need to be adjusted to each other accordingly; cross-talk between them is therefore likely. The most interesting aspect about it, is the regulation of CdaA by the phosphoglucosamine mutase GImM.

GImM has been found to interact with the DAC CdaA in Bacillus subtilis, Lactococcus lactis, Staphylococcus aureus and we could confirm the interaction for L. monocytogenes (Gundlach et al., 2015b; Tosi et al., 2019; Zhu et al., 2016; chapter 2). In all these organisms GImM and CdaA show a physical interaction. To investigate the effect of GImM on CdaA in vivo without impact of CdaR or the PDEs, CdaA and GImM were co-expressed with the c-di-AMP regulated KimA potassium transporter. A potassium transporter deficient strain was grown with specific potassium concentrations and expressed the KimA transporter from L. monocytogenes was expressed (see chapter 3). Growth of Escherichia coli in this screening system is dependent on the uptake of potassium, which can be inhibited by c-di-AMP if a DAC is expressed. We could demonstrate that co-expression of GImM and CdaA impact c-di-AMP synthesis if expressed in E. coli. GImM is able to inhibit CdaA, as seen by the improved growth rate (Fig. 2.7), which has been also shown for L. lactis and S. aureus. In L. lactis, GImM can inhibit CdaA and a suppressor mutation in the c-di-AMP elevated $g d p P$ mutant that restored osmotic resistance were identified to be loss- and gain-of function mutations in $c d a A$ and $g d p P$, respectively. In one case an amino acid exchange in the essential GImM enzyme (I154F) appeared. This mutation had two effects, a decrease in cellular c-di-AMP concentration and at the same time a reduction in the PG precursor UDP-N-acetylglucosamine (UDPNAG). UDP-NAG levels were higher in the $g d p P$ mutant, demonstrating that UDP-NAG concentrations seem to be impact by the c-di-AMP concentration. The authors furthermore could show that the I154F GImM mutant lead to a stronger inhibition of CdaA, directly linking enzymatic activity for cell wall precursor synthesis and osmoregulation by c-di-AMP synthesis (Zhu et al., 2016). Interestingly, the $154 \mathrm{~F}$ mutation is a natural variant in the GImM homologs of B. subtilis, L. monocytogenes and S. aureus, indicating a naturally stronger GImM-dependent inhibition of CdaA activity in these bacteria. The second study in $S$. aureus demonstrated three very interesting details about CdaA regulation: (i) Tosi and colleagues could show that CdaA and GImM both form dimers, interact with each other and that multi-homodimers of CdaA need to interact for enzymatic function. (ii) They furthermore demonstrated that GImM interaction with $\mathrm{CdaA}$ disturbs this 
multimerization and thereby inhibits c-di-AMP synthesis. (iii) the effect of GlmM seems to be stronger than the effect of CdaR on CdaA activity in S. aureus, compared to L. monocytogenes (chapter 2; Tosi et al., 2019). There are however a few questions that remained unanswered and should be addressed in future studies. Co-localization of GImM and CdaA in vivo, effect on GlmM activity on CdaA activity and investigation of $\mathrm{GlmM}$ from other organisms that do not possess c-di-AMP have the same effects. In L. monocytogenes CdaR seems to be able to decrease c-di-AMP concentrations to a level where KimA activity is no longer impaired, while GImM only achieves an intermediate effect, although the strongest effect is seen when CdaR and GImM are present (Fig. 2.7). The most likely reason for this difference may be an evolutionary adaption of c-di-AMP regulation that differs in coccoid bacteria, such as $S$. aureus, Streptococcus pneumoniae or L. lactis from rod-shaped bacteria, such as B. subtilis or L. monocytogenes. In both bacteria, CdaR consists of an amino-terminal transmembrane (TM) domain and four YbbR domains of unknown function. In contrast to that, the coccoid bacteria have CdaR variants with only three or two YbbR domains or mutated pseudogenes, like in L. lactis (Rismondo et al., 2016; Zhu et al., 2016). This hints at a divergent evolution between rod-shaped and coccoid bacteria for the regulation of CdaA activity. Intriguingly the role of $\mathrm{CdaR}$ itself in regulating $\mathrm{CdaA}$ activity might be the key to explain this difference. While GImM seems to play a role in regulation of DAC activity of CdaA, there are still open questions about this regulatory process. In $B$. subtilis, both $\mathrm{G} \operatorname{lmM}$ and $\mathrm{CdaA}$ are expressed equally and constitutively, which indicates that another factor, like enzymatic activity of GImM has to influence the inhibition of CdaA by GlmM. It is also unclear, if enzymatic activity or other factors influence co-localization and interaction of $\mathrm{G} / \mathrm{mM}$ with $\mathrm{CdaA}$ in vivo and needs to be investigated in future studies.

\section{Regulation of CdaA by CdaR}

CdaR has been shown to effect CdaA activity in B. subtilis, L. monocytogenes and to lesser extend also in S. aureus (Mehne et al., 2013; Rismondo et al., 2016; Tosi et al., 2019). CdaR, called YbbR in $S$. aureus, has also been linked to an increased acid susceptibility in this bacterium. In the study by Bowman and colleagues, a cdaR mutant did not show significantly increased c-di-AMP concentrations under the used conditions, but an increased acid sensitivity and accumulated suppressor mutations that circumvented this phenotype (Bowman et al., 2016). Mutations occurred, among others, in genes encoding for a putative proline/betaine transporter or the gene encoding for the DAC CdaA (DacA in S. aureus). Interestingly, growth assays showed, that the cdaR mutant phenocopied the growth defect of a low c-di-AMP cdaA (G206S) mutant under acidic conditions, indicating that under acidic stress a $c d a R$ mutant might show lower c-di-AMP concentrations than the wild type. Surprisingly, although c-di-AMP concentrations did increase with acidic stress, they increased even further in the cdaR mutant and even more so in the suppressor mutants. This increase, however, was observed also at neutral $\mathrm{pH}$, although more prominent for the suppressor strains than the cdaR mutant alone (Bowman et al., 2016). In L. monocytogenes on the other hand, a cdaR mutant showed about $40 \%$ elevated c-di-AMP concentration under growth in complex medium and a $10 \%$ decrease if CdaR was overexpressed, indicating inhibition of CdaR under the used conditions. Surprisingly, a cdaR mutant did not show an altered resistance towards cell wall-acting antibiotics but an increase resistance towards lysozyme (Rismondo et al., 2016). Fascinatingly, in a study by Wichgers Schreur and colleagues, lysozyme resistant mutants were found to have a mutation in the CdaA homolog of Streptococcus suis (Wichgers Schreur et al., 2012). We pursued this interesting link between c-di-AMP metabolism and cell wall biosynthesis by evolving L. monocytogenes to become more lysozyme resistance (chapter 6 ). Interestingly, all suppressor mutants 
evolved mutations in the promoter region of a small RNA (rli31) that is known to effect PG remodeling by regulating abundance of the PG deacetylase PgdA and the putative carboxypeptidase PbpX by a still to be elucidated mechanism (Burke et al., 2014; Burke \& Portnoy, 2016). The cell wall-related phenotypes of c-di-AMP metabolic mutants might, however, be at least in part indirect effects, due to an altered osmoregulation (Commichau et al., 2018). We could show that CdaR on the one hand is indeed a membrane localized protein and that the YbbR domains are located outside of the cell (Fig. 2.2 \& Fig. 2.3). This eliminates the hypothesis that the YbbR domains may interact with the DAC domain directly, as some unusual DAC-YbbR domain hybrid enzymes, like CdaZ of the methanogenic archaeon Methanocaldococcus jannaschii might indicate and is in good accordance with a BACTH study, showing self-interaction of the DAC domain of CdaA and the YbbR domains of $\mathrm{CdaR}$, but only interaction of $\mathrm{CdaA}$ and $\mathrm{CdaR}$ in their full-length, TM domain containing, variants (Commichau et al., 2019; Kellenberger et al., 2015; Rismondo et al., 2016). We could, furthermore, directly link CdaR to metabolic activity and normal growth of $L$. monocytogenes under osmotic stress conditions, demonstrating its putative role as a sensor for changes in osmolarity for regulating CdaA activity (Fig. 2.1). After identifying the role of CdaR on osmoregulation, we set out to elucidate how the protein might act as an osmolarity sensor. There have been three main hypotheses: (i) CdaR might sense cell wall alterations and convey the signal input on CdaA. (ii) CdaR might act as a proper localizer for CdaA, facilitating self-interaction and/or interaction with other proteins. (iii) CdaR might act as a signal amplifier for cell envelope stress and influence proper interaction and localization of CdaA with itself and/or other proteins.

The basis of these models is derived from known mechanisms of cell-wall interacting signal transduction protein, like PrkA, combined with our current knowledge of CdaR and osmotic regulation in general. To further elucidate: (i) CdaR might sense the constitution of the cell wall. Similar functions are known from PrkA-like proteins (PrkC in B. subtilis). PrkA is a TM protein with an intracellular kinase domain and three $\mathrm{C}$-terminal and extracellular PASTA domains (for Penicillin binding proteins and serine/threonine kinase associated; Manuse et al., 2016). Those domains have been shown to bind peptidoglycan (PG) and possible detect specific changes in PG composition and in turn alter activity of the intracellular kinase domain. Increased phosphorylation of its targets WalR, CpgA, YvcK and GpsB leads to increased expression of the walR regulon and alters activity of YvcK, CpgA and GpsB, respectively, eventually leading to an altered cell wall architecture and metabolism. One has to note though that the function of and the signals that are perceived by PASTA domain containing kinases in various organisms are not fully understood and might have evolved differently in different bacteria (Manuse et al., 2016). This might be also true for CdaR. The abovementioned differences in YbbR domain repeats between different bacteria might suggest as much. In addition, structural analysis has shown that YbbR domains share a common fold, while having a rather un-conserved amino acid similarity (Barb et al., 2010). This might hint at a general role of them as PG interacting, or self-interacting proteins with distinct differences from species to species and would be an interesting subject for further studies. This led to the hypothesis that CdaR might act as a membrane anchor or localizer for CdaA (ii). In this model, the YbbR domains would determine the location of $\mathrm{CdaA}$, via protein-protein interaction with the TM domains. The YbbR domains could determine the localization of the complex, e.g. at the septum to facilitate interactions with other proteins, leading to clustering of functional complexes. Interaction of CdaA, but especially CdaR in a two-hybrid assay (Fig. 2.6) would be in favor of this model. There is however the question how alteration of the osmolarity would impact CdaA activity in a CdaR-dependent matter. CdaR could either regulate CdaA self-interaction, if the strain in the cell wall is large enough to alter the putative interaction between the YbbR domains and the cell wall (with CdaR acting as an anchor for CdaA and PDEs, stabilizing complex formation and thereby altering activity) 
or CdaA and the PDEs might have osmosensing abilities that are at least in part independent of $C d a A$. This model could be indeed the case, considering that $L$. lactis has no functional cdaR gene and that the phenotypes of a cdaR mutant in L. monocytogenes on osmotic adaption (Fig. 2.1) are not as strong as it has been shown for PDE mutants (Huynh et al., 2016; Zhu et al., 2016; chapter 2). A possible mechanism for CdaA and the PDEs to at least partially sense osmotic stress on their own is given by the recent proposed model of osmosensing by the PhoQ/PhoP two-component system in E. coli (Yuan et al., 2017). It has been shown that the two-component system responds to an osmotic upshift via the PhoQ kinase. Kinase mutants lacking the periplasmic sensor domain, which is important for PhoQ to detect other environmental clues, were still responsive to changes in osmolarity. The authors could show, that osmotic activation is dependent on the length of the TM helices and lateral pressure through the membrane. In their proposed model, the lateral pressure leads to conformational changes in the TM domain that in the end alter catalytic activity of the cytosolic kinase domain (Yuan et al., 2017). A similar model could be possible for CdaA, PdeA or $\mathrm{PgpH}$ that are all membrane-bound proteins with cytosolic enzymatic domains, with a huge contribution to osmoregulation in Firmicutes. Another possible clue are of course alterations in ion concentrations. Interestingly, in vitro a truncated variant of $L$. monocytogenes or S. aureus CdaA is most active with cobalt or manganese (cobalt predominantly for L. monocytogenes and manganese for $S$. aureus as a cofactor (Rosenberg et al., 2015; Tosi et al., 2019). DACs of the DisA family usually show highest activity with magnesium as a cofactor and CdaA from $S$. aureus also shows some activity with magnesium, rising the questions if the natural cofactor of full-length CdaA variants in vivo might be magnesium after all (Tosi et al., 2019; Witte et al., 2008). If cobalt and/or manganese are the natural cofactors for cyclases of the CdaA type, their availability and competition with the intracellular much higher concentrated magnesium ions could contribute to regulation of DAC activity, depending on their different affinities, concentrations and alterations due to osmotic stress (Foster et al., 2014; Groisman et al., 2013). The third model is a combination of the first two. (iii) CdaR might have two different functions. On the one hand CdaR might determine localization and/interaction of $\mathrm{CdaA}$ with itself and putatively other components of the c-di-AMP metabolism and on the other hand modulate self-sensing properties by acting as an enlarged interaction surface with the cell wall two magnify signal input. In this model CdaA and PDEs would not be fully dependent on CdaR, but presence of CdaR would facilitate the magnitude of DAC activity and finetune it (see Fig. 2.8).

\section{The role of the YbbR domains of the diadenylate cyclase regulator CdaR}

To investigate how CdaR might sense changes in osmolarity and convey these signals to CdaA, to modulate its activity, we took a closer look at the function of the YbbR domains and the membrane localization of CdaR in vivo. As seen in Fig. 2.5, a cdaR mutant is still able to adjust its intracellular c-di-AMP concentration, but shows in general a decrease of c-di-AMP compared to the wild type and the range of adaptation seems to be decreased. A mutant expressing CdaR without its TM domain behaves like the cdaR deletion mutant. Taken together with the topology assays that showed that the TM domain is both, necessary and sufficient for correct membrane localization of the adjacent C-terminus (Fig. 2.3), it can be stated that extracellular localization of the YbbR domains is necessary for proper CdaR-dependent regulation of CdaA activity. This is especially of interest considering the effect of a mutant expressing CdaR without any YbbR domains, meaning only the TM domain. In this mutant we could only measure about a fifth of the c-di-AMP amount compared to the wild type strain. We concluded from this phenotype that expression of the TM domain without the YbbR domains disturbs $\mathrm{CdaA}$ dimerization. Considering the before mentioned 
three hypotheses that CdaR might (i) sense cell wall alterations, (ii) act as a localizer and anchor for $\mathrm{CdaA}$ or (iii) act as a scaffolding protein with cell envelope signal amplifying function, to stabilize CdaA self-interaction, model two or three are in good agreement with the observed phenotypes. If CdaR would sense alterations in cell wall composition, the effect of $\mathrm{CdaR}$ should be slight conformational rearrangements of $\mathrm{CdaR}$ and in consequence of $\mathrm{CdaA}$ and thereby specific changes in activity upon YbbR domain interaction with an altered cell wall. A mutant without the sensory YbbR domains should therefore behave like the wild type, except when a signal is perceived. In contrast to that, expression of YbbR-less CdaR impacts CdaA activity per se. In the other two models, where CdaR is important for proper interaction and/or localization of CdaA, the YbbR domains would be a determinant for self-interaction. It has been shown that the YbbR domains are able to self-interact in the cytosol, if the TM domains are truncated and, furthermore, that CdaA selfinteracts, although weaker in the full-length variant compared to mutants lacking TM domains. Moreover, CdaA and CdaR only interact with each other as full-length proteins, presumably via their TM domains (Rismondo et al., 2016). Hence, it can be hypothesized that CdaA and CdaR selfinteractions might be influenced by their membrane localization in a negative way. Considering this, expression of CdaR TM domain that interacts with the TM domains of CdaA could destabilize CdaA self-interaction, if not stabilized by YbbR domain interaction. The phenotype of the YbbRless CdaR expressing L. monocytogenes strain is in support of this hypothesis (Fig. 2.5). Interestingly, a complementation strain shows the opposite phenotype of the cdaR deletion mutant. Higher c-di-AMP concentrations and a greater change upon osmotic change can be detected, which is in agreement with the proposed model (see Fig. 2.8).

When we compared our experimental findings to what is known about the impact of CdaR on the cellular c-di-AMP concentration in L. monocytogenes, we noticed two interesting differences. On the one hand, effects of $c d a R$ deletion and complementation lead to decreased and increased c-di-AMP level, respectively, which is diametrically opposed to what is known from previous experiments and on the other hand c-di-AMP concentrations were in general lower than previously observed (Rismondo et al., 2016). The experimental procedures of these two experiments were distinct by two factors that may be the root for the differences. In Rismondo et al., the bacteria were cultivated in $\mathrm{BHI}$, a very nutrient rich complex medium and c-di-AMP concentrations were measured at an $\mathrm{OD}_{600}$ of 1 , the late exponential growth phase (the c-di-AMP concentration in the wild type, which was set to $100 \%$ was $99.4 \pm 13.4$ ng c-di-AMP per mg of protein; Gibhardt, 2015; Rismondo et al., 2016). In contrast to this set up, we determined c-di-AMP concentrations in LSM, a defined medium and at an $\mathrm{OD}_{600}$ at the early- and mid-exponential phase. It is not known, how c-di-AMP concentration may change upon osmotic stress in dependency of the growth phase, but it is known from c-di-AMP and also other second messenger molecules, c-di-GMP that plays a role in $E$. coli lifestyle changes from exponential to the stationary growth phase that the growth phase is a determinant for nucleotide second messenger concentrations (Hengge et al., 2015). Similar as for c-di-AMP, the main determinants for the intracellular c-di-GMP concentration are synthesis and degradation by specialized cyclases and PDEs. Interestingly, expression of the PDE PdeH is inhibited and expression of the diguanylate cyclase DgcE increased during the entry into the stationary phase of $E$. coli and together with other factors the increase in c-di-GMP inhibits motility and facilitates adhesion and matrix production of E. coli in the stationary phase (Hengge et al., 2015; Pesavento et al., 2008). For c-di-AMP, there are studies that demonstrate a cross-talk with the (p)ppGpp regulated stringent response, leading to altered concentrations of c-di-AMP, while the ( $\mathrm{p}$ )ppGpp concentration depends on the growth phase and nutrient availability (Corrigan et al., 2015). Corrigan and colleagues could show for $S$. aureus that c-di-AMP levels are indeed rising in late growth phases and that gene expression patterns of cells with a high c-di-AMP 
concentration overlap in parts with those of cells undergoing the stringent response. They furthermore could show that high c-di-AMP levels activate the RelA enzyme, leading to an increase in (p)ppGpp by an indirect and unknown mechanism (Corrigan et al., 2015). Interestingly, other studies have also demonstrated inhibition of c-di-AMP PDEs by (p)ppGpp, indicating a tight crossregulation of those two nucleotide second messenger pathways that might play a role in osmoregulation and will be discussed further below (Corrigan et al., 2015; Huynh et al., 2015; Rao et al., 2010; Whiteley et al., 2015). To conclude, c-di-AMP levels in L. monocytogenes in the early exponential phase might, like in S. aureus, may be lower compared to later growth phases. Fascinatingly, cellular turgor is, through the accumulation of intracellular osmolytes, which is favored by lower c-di-AMP concentrations, a determinant for cell division in Gram-positive bacteria. In the exponential phase osmolyte import in general has to be controlled tighter, to prevent an excessive increase in turgor that might be detrimental and therefore favors higher c-di-AMP levels (Rojas et al., 2018). The second big difference in the experimental set ups was the medium composition. In a complex medium like $\mathrm{BHI}$, in which a multitude of ionic and peptide osmolytes are present. In contrast to that, the Listeria synthetic medium (LSM) features defined amounts of amino acids, trace elements, vitamins and low amounts of potassium $(4.8 \mathrm{mM})$ and, therefore, compared to a complex medium like BHI or LB a limited, defined amount of low complexity osmotic active substances (Whiteley et al., 2017). The key difference might be the presence of oligopeptides in complex media that act as osmolytes in L. monocytogenes and are at least indirectly regulated by the impact of c-di-AMP on the stringent response and thereby expression of oligopeptide permeases (Whiteley et al., 2015; Whiteley et al., 2017). In a minimal medium, lower amounts of c-di-AMP are needed to balance the import of the less abundant osmolytes, like it has been demonstrated for potassium uptake and c-di-AMP levels in B. subtilis (Gundlach et al., 2017).

The different media compositions and or growth phase might also have an impact on the role of $\mathrm{CdaR}$ as a regulator. We therefore hypothesized that $\mathrm{CdaR}$ can act on $\mathrm{CdaA}$ as an inhibitor and activator, meaning it can in general modulate the activity of CdaA, in dependency of different stimuli. Nevertheless, additional experiments in E. coli provided an alternative hypothesis. Using the before mentioned E. coli- and KimA transporter-based c-di-AMP reporter system, we investigated impact of CdaR and CdaR variants on CdaA activity. Interestingly, in E. coli, CdaR seems to inhibit CdaA activity if it is membrane localized and at least one YbbR domain is present. In contrast to the findings in L. monocytogenes, the mutant lacking all YbbR domains did not show a growth phenotype corresponding to low c-di-AMP, but rather showed an intermediate phenotype, similar to GlmM, meaning it is still able to act in part as an inhibitor on CdaA. This difference of phenotype in L. monocytogenes and $E$. coli can be a consequence of the differences between the Gram-positive and Gram-negative cell envelope, since the CdaA-CdaR complex would presumably be located in the inner membrane of $E$. coli and the YbbR domains therefore present in the periplasm. Furthermore, different compositions in the lipid bilayer, composition of PG or interactions with other proteins could influence $\mathrm{CdaA}$ activity and the impact of regulator molecules on its activity (Silhavy et al., 2010). Another difference and is the lack of other proteins of the c-di-AMP signaling pathway in E. coli. Usually this is an advantage for studying DAC activity and the influence of single regulators on cyclases, but in this case we were wondering if other components of the signaling pathway might have an influence on CdaA activity or the role of CdaR. To elucidate this, we employed a bacterial two hybrid protein-protein interaction assay and indeed found protein-protein interactions between synthesis and degradation machineries (Fig. 2.6). It is therefore possible that $\mathrm{CdaA}$ and $\mathrm{CdaR}$ might be influences by PdeA and $\mathrm{PgpH}$ and vice versa and this potential cross-talk and the resulting local signaling should be the topic of further studies. 


\section{Regulation of other diadenylate cyclases}

In contrast to L. monocytogenes, B. subtilis has two other types of cyclases - DisA and CdaS. CdaS is sporulation specific expressed and only implicated in spore formation and germination with a putative role of its autoregulatory $\mathrm{N}$-terminal helices in regulating its activity (Mehne et al., 2013; Mehne et al., 2014; Zheng et al., 2015).

In light of the recent implications of c-di-AMP being a major regulator of osmotic homeostasis in bacteria, we challenged the so far known model of DisA regulation and investigated the hypothesis that DisA activity might me controlled by changes in osmolarity. Interestingly there are several examples of osmotic stress altering the DNA-binding of proteins. It has been shown that interaction of the RNA polymerase with the DNA is released after condensation of the DNA due to macromolecular crowding during the initial phase of a hyperosmotic shock (Cagliero et al., 2013; Wood, 1999). Another example is the interaction between the transcriptional repressor of the glycine betaine importer encoding busAA-busAB operon by BusR. BusR interaction with the RNA polymerase-DNA complex is inhibited by increased ionic strength, e.g. increased potassium ion concentration after the first response to a hyperosmotic stress, allowing expression of the glycine betaine transporter for import of compatible solutes as a secondary response (Romeo et al., 2007). Intriguingly, BusR has also been identified as a c-di-AMP binding protein in S. agalactiae (Devaux et al., 2018), highlighting an additional layer of regulation.

The second vegetative DAC of $B$. subtilis, DisA, is present in vegetative cells and regulated by its interaction with DNA (Witte et al., 2008). It is the second most abundant DAC and present in many spore-forming Firmicutes and as sole DAC in many Actinobacteria, but interestingly, c-di-AMP is not essential in Actinobacteria, like Mycobacterium tuberculosis, containing DisA as a sole cyclase (Yang et al., 2014; Commichau et al., 2015). DisA has been implicated to scan the DNA for damages, like double strand breaks, stall and being inhibited upon contact with such structures (Bejerano-Sagie et al., 2006; Oppenheimer-Shaanan et al., 2011; Witte et al., 2008). Furthermore, its interaction with other proteins involved in DNA repair mechanisms have supported this hypothesis (Gándara et al., 2017; Zhang \& He, 2013). There have, however, been observations that contradict the hypothesis of DisA being a major factor in regulation of DNA damage repair. A B. subtilis mutant can grow normally if only one c-di-AMP producing enzyme is present without showing instability due to accumulation of mutations, indicating that the nucleotide itself is the determinant for normal cellular growth and not the producing enzyme (Mehne et al., 2013; Gundlach et al., 2017). Closely related bacteria, like L. monocytogenes or S. aureus harbor only one cyclase of the CdaA-type (Corrigan et al., 2011; Commichau et al., 2015; Rismondo et al., 2016; Rosenberg et al., 2015). Moreover, as afore mentioned, in Actinobacteria that often harbor DisA as the sole DAC, the corresponding gene is not essential (Commichau et al., 2015; Yang et al., 2014). Taken together, this does not exclude a function of DisA in DNA repair, but it suggests that it is not its main regulatory function.

As described in chapter 5 , interaction of DisA with chromosomal DNA inhibited its activity in a DNA concentration-dependent matter (Fig. 5.1). Additionally, an increase in the concentration of ionic osmolytes, such as potassium or sodium, but not the nonionic saccharide sucrose, released the inhibitory effect of DNA on DisA activity (Fig. 5.2). We could further demonstrate that a DisA variant with a mutation that decreased DNA-binding (G334E; Witte et al., 2008) is more active than the wild type variant in vivo, but not in vitro (Fig. S5.2 \& Fig. 5.3, respectively). Interestingly, in vivo c-di-AMP measurements showed that upon osmotic stress with $\mathrm{NaCl}$ the c-di-AMP concentration first decreases and then increases (Fig. 5.5). This allows the cell to accumulate osmolytes 
as a counter measure and afterwards prevents excessive accumulation. Comparing the G334E mutant with the wild type DisA, there is no difference between time point one (shortly after the hyperosmotic shock) and time point two (when the cells began to resume growth after a stagnation). We presume that the G334E mutant might replenish the c-di-AMP pool faster than the wild type, which seems indeed to be the case if the cells are stressed with $\mathrm{KCl}$ (Fig. 5.5). Interestingly, c-di-AMP levels seem to stay stable after the osmotic stress and then rise, with the mutants reaching higher concentrations compared to the strain harboring wild type DisA. In the case of potassium as a stressor it makes sense for the cells to not lower the c-di-AMP concentration to prevent excessive accumulation of potassium via c-di-AMP regulated potassium transport systems (Gundlach et al., 2017). B. subtilis must therefore have developed mechanisms to distinguish if the osmotic stress is caused by ions or other solutes and in case of ions also between the ions. DisA could be a candidate to discriminate between different ions. It has been shown that the affinity of DNA to the most prevalent ions in the cytoplasm is highest for calcium ions, followed by magnesium, potassium and sodium, with the latter two having similar affinities (Korolev et al., 1999). Interestingly the study also described that under physiological concentrations of the different cations they show similar degrees of DNA-binding. This means that potassium ions can outcompete the stronger binding calcium ions due to the higher concentration of potassium ions, opening the opportunity that changes in intracellular ion concentration due to osmotic stress could transiently change the balance of DNA-ion interaction. This might be the reason why we see a difference on DisA-DNA inhibition and ion addition in vitro, compared to the in vivo measurements. In vitro $\mathrm{NaCl}$ and $\mathrm{KCl}$ addition both release DisA inhibition by DNA, while in vivo the influx of the ions would be a major determinant, meaning the rate of influx of the ions in the cytoplasm. Since potassium and not sodium uptake, as an osmolyte, is known to be the first response of bacteria to a hyperosmotic shock, the influx of potassium should in vivo have a stronger impact on DNA-binding proteins and is in line with our observations (Fig. 5.5). Interestingly, in vitro studies of DisA G334E activity showed that it is as strongly inhibited by chromosomal DNA as the wt DisA variant, but release of inhibition is slightly altered, meaning a lower potassium concentration seems to be sufficient to let DisA dissociate from the DNA (Fig.5.4). A reason for this could be that the G334E mutation affects binding to certain DNA structures more than the general binding to DNA and other factors like macromolecular crowding, competition with other DNA-binding proteins might affect the impact of the mutation in vivo.

Considering that DisA is not the sole cyclase in B. subtilis a system of CdaA sensing turgor changes and DisA sensing changes in macromolecular crowding and DNA-ion effects may have evolved. The CdaA system might be sufficient for bacteria to adapt to osmotic stress, sensing fundamental physical changes that occur independent of the osmotic stressor and the DisA system may have evolved as a fine tuning or special adaptation. Actinobacteria with DisA as a sole DAC might therefore have other, c-di-AMP-independent osmoregulatory systems that detect turgor changes as in Proteobacteria, like E. coli (Sleator \& Hill, 2002; Wood, 1999).

\section{c-di-AMP-regulated osmotic homeostasis in Listeria monocytogenes}

Regulation of osmotic homeostasis has been shown to be the major determinant of c-di-AMP essentiality in Firmicutes (Gundlach et al., 2017; Whiteley et al., 2015; Whiteley et al., 2017; Zeden et al., 2018). Uptake of potassium is the first response of bacteria to a hyperosmotic shock, but the set up and characteristics of potassium transport is not well studied in L. monocytogenes. As described in chapter 3 , homologs of the B. subtilis low affinity KtrCD transporter and the high 
affinity KimA transporter, as well as a homolog to the $S$. aureus two component system KdpDE and the transcriptional controlled KdpABC high affinity transporter were identified. Our main interest in studying the potassium transport processes was the investigation of c-di-AMP-dependent regulation, as it has been shown for B. subtilis KtrAB and the development of a c-di-AMP reporter system, suitable for in vivo antibiotic screening (Corrigan et al., 2013; Kim et al., 2015). We identified that the homologs of KtrCD and KimA of L. monocytogenes are indeed low and high affinity potassium transporters, respectively, with their affinities comparable to previous findings (Gundlach et al., 2017; Holtmann et al., 2003). We also identified KtrC as a novel c-di-AMP binding protein in L. monocytogenes and that both KimA and the KtrCD transporter are inhibited by c-di-AMP in vivo (Fig. 3.7 and Fig. 3.6, respectively). Interestingly, we also observed a strong phenotype if the potassium transporters were overexpressed in E. coli. Overexpression resulted in osmotic swelling of bacteria, highlighting the importance of regulated osmolyte influx (Fig. 3.5). This phenotype is in excellent agreement with previous observations that lack of c-di-AMP leads to osmotic swelling and consequently to lysis of the bacteria, while high c-di-AMP levels lead to an aberrant curli morphology that can be explained by a decrease in intracellular osmolytes and subsequent water-loss (Gundlach et al., 2017; Mehne et al., 2014). These osmoregulation related phenotypes highlight the importance of a well-balanced uptake and regulation of osmolytes, where c-di-AMP plays a major role in Firmicutes. As summarized in Fig. 7.1 and in comparison, to Fig. 1.3, the knowledge about osmoregulatory systems and the role of c-di-AMP in L. monocytogenes and other bacteria have greatly increased.

c-di-AMP has been identified to regulate various osmolyte transport systems in Firmicutes. It regulates potassium uptake in B. subtilis, S. aureus, S. pneumoniae, L. lactis and L. monocytogenes (Bai et al., 2014; Corrigan et al., 2015; Gundlach et al., 2017; Pham et al., 2018; Quintana et al., 2019; chapter 3). Besides the regulation of potassium homeostasis, c-di-AMP has also been implicated in the regulation of glycine betaine, oligopeptide and carnitine uptake in several different bacteria that as compatible solutes all play an important role in osmotic adaptation (Commichau et al., 2018; Devaux et al., 2018; Huynh et al., 2016; Pham et al., 2018; Schuster et al., 2016; Whiteley et al., 2015; Whiteley et al., 2017).

It can therefore be state that c-di-AMP is the major regulator for osmoregulation in Firmicutes. As a second messenger and "essential poison" c-di-AMP levels need to be tightly adjusted (Gundlach et al., 2015b). Due to its regulation of osmotic homeostasis its synthesis and degradation must react to changes in osmolarity. We could show that both DACs CdaA from L. monocytogenes and DisA from $B$. subtilis indeed are regulated by changes in osmolarity (chapters 2 and 5 , respectively). CdaA might thereby be, in dependency of CdaR, a turgor-reactive DAC, while DisA senses macromolecular crowding and the intracellular ion composition. While CdaA seems to connect osmotic adaptation to cell wall biosynthesis via GImM, DisA might connect osmotic stress to cell cycle arrest and facilitating stress responses like DNA repair mechanisms via RadA.

While many aspects still need to be elucidated, the present studies shed new insight in the regulation of c-di-AMP synthesis and might also provide new insight into regulation of degradation and a phenomenon termed local signaling for the second messenger c-di-GMP (Sarenko et al., 2017). Like for c-di-GMP, it can be imagined for c-di-AMP that a certain intracellular concentration is present in dependency of the environmental conditions, with local pools of higher or lower concentrations. Such a local signaling can be achieved by specific co-localization of synthesizing and or degrading enzymes with receptor molecules (DNA, RNA or proteins). It would allow a fine tuning and fast response of membrane transport if diffusion distances for the second messenger would be reduced in this way. 


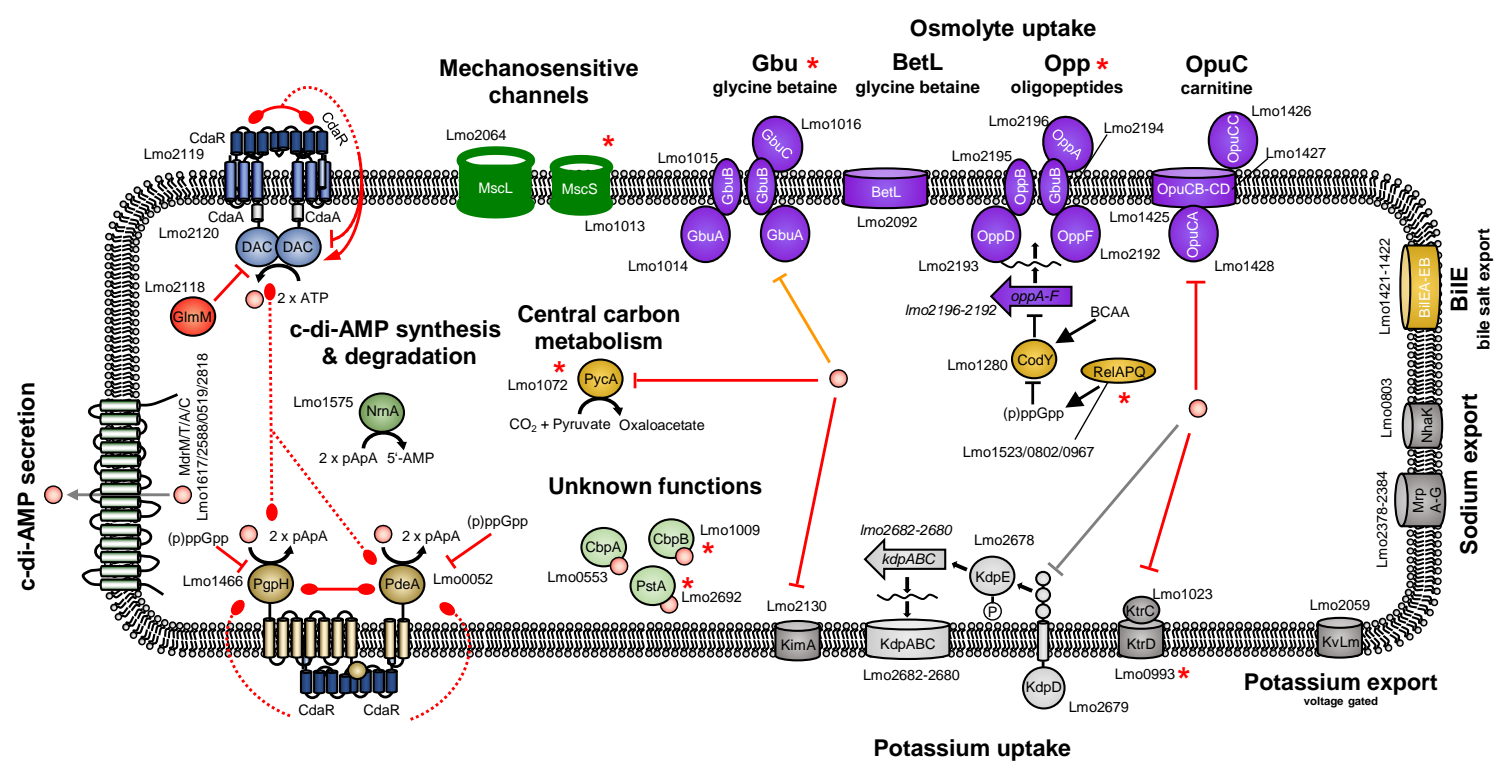

Fig. 7.1 The current knowledge about c-di-AMP signaling and osmoregulation in L. monocytogenes. c-di-AMP is synthesized by the sole DAC of $L$. monocytogenes, CdaA. CdaA is controlled by the two regulatory proteins CdaR and GImM. Our current knowledge is depicted as self-interaction of the YbbR domains modulating CdaA activity in dependency of the strain in the cell envelope. The effects of GImM on CdaA activity seem to be less pronounced than the effects of $\mathrm{CdaR}$ in L. monocytogenes compared to $S$. aureus and vice versa. L. monocytogenes possesses multi drug resistance efflux transporter that can secrete c-di-AMP and might, like the phosphodiesterases (PDEs) PdeA and PgpH contribute to rapid decrease of intracellular c-di-AMP. Protein-protein interaction experiments, furthermore hint to an interaction between PDEs, CdaA and CdaR. L. monocytogenes also possesses homologs of a nano-Rnase (NrnA) and two mechanosensitive channels (MscL and MscS) that might contribute to c-di-AMP degradation and osmoregulation, respectively. c-di-AMP also inhibits the pyruvate carboxylase PycA and binds to the proteins of unknown function $\mathrm{CbpA}, \mathrm{CbpB}$ and PstA. L. monocytogenes furthermore possesses osmolyte uptake system for glycine betaine (Gbu and BetL), oligopeptides (Opp), carnitine (OpuC) and a bile salt specific exporter (BilE). While OpuC has been shown to be directly inhibited by c-di-AMP, Opp and Gbu are indirectly implicated by occurrence of suppressor mutations in L. monocytogenes. For Gbu, regulation by c-di-AMP in other bacteria was observed. The Opp system is also indirectly linked to c-di-AMP homeostasis due to the cross-talk between the stringent response and c-di-AMP and the occurrence of c-di-AMP-dependent suppressor mutations in the RelA enzyme. L. monocytogenes also possesses several homologs of ion transport systems, among them the voltage gated KvLm potassium exporter and potassium import systems. Of those, the KdpD protein is known to bind c-di-AMP in S. aureus and regulate expression of the KdpABC high affinity transporter. KtrAB, is regulated by c-di-AMP on the protein level in S. aureus, as we demonstrate also for KtrCD of L. monocytogenes. Last but not least, we could identify KimA as a potassium uptake system in L. monocytogenes and show for that also this class of potassium transporter is inhibited by c-di-AMP (based on Commichau et al., 2018).

Moreover, this would allow interesting fine-tuning mechanisms where the activity of the receptor molecule would directly impact the activity of DAC or PDE and it would lead to even more complex regulatory networks. Our protein-protein interaction study (chapter 2 ) does indeed hint at the possibility of protein-protein interaction between components of the synthesis, degradation machineries and targets of c-di-AMP signaling and it will be interesting to study the occurrence and possible regulatory consequences of these interactions and the impact of c-di-AMP on novel targets in future studies.

\section{c-di-AMP affects global gene expression and protein biosynthesis}

One important aspect of future studies on c-di-AMP signaling will be the identification and elucidation of the regulation of novel c-di-AMP regulated processes. As described in chapter 4 , we investigated the effect on $L$. monocytogenes if its sole DAC encoding gene $c d a A$ is deleted under 
defined growth conditions. We identified a total of 95 genes and 21 proteins that are at least 2or 1-5-fold differently regulated, respectively, compared to the wild type strain. Among these were many genes involved in cellular processes like replication or transcription, carbon and nitrogen metabolism, signal transduction processes, cell wall and membrane biogenesis, motility and genes of unknown function. As one can immediately deduct from this list, c-di-AMP impacts a lot of different cellular processes. The big remaining question here is if these processes are directly regulated by c-di-AMP or indirect consequences due to the altered osmotic equilibrium this strain probably has.

As described above, c-di-AMP signaling shows cross-talk with other cellular processes, like the stringent response, cell wall biosynthesis or DNA-related mechanism. It is therefore likely that the changes in gene expression and protein biosynthesis are at least in part indirect effects. Interestingly, among the differently expressed genes also 15 operons with at least two impacted genes were differently expressed, hinting at transcriptional regulation of these operons either directly or indirectly by c-di-AMP. Among those are for example genes involved in cystine/cysteine metabolism, cell division and cell wall biosynthesis, purine biosynthesis and two operons involved in synthesis of flagellar subunits. Interestingly motility in L. monocytogenes is regulated by a mainly ambient temperature-dependent system.

The motility assay we performed did show an impact of c-di-AMP on swim behavior in L. monocytogenes with an increase in the $c d a A$ deletion mutants at $37^{\circ} \mathrm{C}$ (Fig.4.4). This phenotype was not observed as pronounced at $25^{\circ} \mathrm{C}$. It is however unclear, if the difference in swim distance is due to the slower growth or decreased motility and how motility genes are expressed in the cdaA mutant at $25^{\circ} \mathrm{C}$. Interestingly, a $B$. subtilis gdpP mutant showed in a transcriptomic study an inhibition of biofilm genes and reduced biofilm formation (Gundlach et al., 2016). In B. subtilis motile and sessile lifestyle are mutual exclusive and regulated by the DNA-binding protein SinR and its interaction with the antagonists Sinl and SIrR (Vlamakis et al., 2013). Indeed, in the high c-di-AMP containing $B$. subtilis $\triangle g d p P$ strain, motility genes, like the gene encoding the flagellin encoding hag were upregulated. Therefore, c-di-AMP may regulate motility differently, between these two closely related bacteria. In other bacteria, like $E$. coli, differentiation between motile and sessile lifestyle is regulated by the "older brother" of c-di-AMP, c-di-GMP that is also present in B. subtilis, but does not control biofilm formation in it (Gao et al., 2013; Hengge, 2009). In L. monocytogenes one important determinant for virulence is also an important determinant for motility. The expression of the flagellin encoding gene, which is called flaA in L. monocytogenes, is repressed at temperatures that signal a host environment $\left(37^{\circ} \mathrm{C}\right)$ and expressed at lower temperatures that signal the presence outside a host organism (Dons et al., 1992; Peel et al., 1988). In this process the orphan response regulator DegU plays an important role (orphan because the gene of the corresponding sensor kinase DegS is absent in the L. monocytogenes genome). DegU inhibits its own expression and it binds the promoter that governs expression of gmaR, encoding the antirepressor to MogR, the repressor of motility genes (including gmaR). The temperature sensitive GmaR protein, as well as the temperature-sensitive GmaR-MogR interaction, eventually regulates motility gene expression (Kamp \& Higgins, 2009; Kamp \& Higgins, 2011; Shen et al., 2006; for more details see chapter 4 and chapter 6 ). The operon of gmaR also contains the motB genes, encoding proteins of the flagellum, which is an additional reason why DegU is essential for motility in L. monocytogenes (Gueriri et al., 2008; Mauder et al., 2008). Interestingly the global regulator CodY that is also implicated in stringent response-dependent regulation of opp expression, has also been shown to regulate, among others, motility genes (Gibhardt, 2015; Lobel \& Herskovits, 2016; Whiteley et al., 2015). It has also been shown that c-di-GMP effects motility in L. monocytogenes. Deletion of all three c-di-GMP PDEs, which leads to higher c-di-GMP levels, lead to increased 
synthesize of an exopolysaccharide that inhibits motility probably by increasing cell aggregation (Chen et al., 2014; Köseoğlu et al., 2015). This, moreover, highlights that the effects of c-di-AMP on regulation of motility gene expression could be different in B. subtilis and L. monocytogenes.

It is unclear, however, if c-di-AMP directly regulates motility expression or if it is via cross-talk with the stringent response or c-di-GMP signaling that both can influence CodY activity by decreasing GTP pool (Elbakush et al., 2018; Geiger \& Wolz, 2014) or if there is a connection between c-di-AMP and the small RNA rli31 that was identified in a suppressor mutant screen on the search for mutants that mimic the effect of CdaR on lysozyme resistance. We could observe an influence of rli31 on motility that may be affected by c-di-AMP signaling (chapter 6 ). Interestingly, while swarming of the CdaR mutant seems to be like the wild type at $25^{\circ} \mathrm{C}$, it was, in contrast to all other investigated strains, strongly inhibited at $37^{\circ} \mathrm{C}$ on LSM plates. Contrarily to previous studies that show strict temperature-dependent motility regulation, the motility assay on LSM plates at $37^{\circ} \mathrm{C}$ displayed a temperature-independent regulation of motility, especially the $c d a A$ deletion strain showed an increased motility (chapter 4). The temperature independent regulation of motility might therefore be influenced by c-di-AMP.

This is just one example of the many research possibilities of finding new c-di-AMP-regulated processes. With the data in hand, we can find new targets of c-di-AMP regulated transcription factors, such as BusR in L. lactis or S. agalactiae or new RNA structures like the ydaO (kimA) riboswitch that governs potassium transporter expression in B. subtilis (Devaux et al., 2018; Nelson et al., 2013; Pham et al., 2018).

Overall, the omics data gathered will help us to expand our understanding of the c-di-AMP signaling network in L. monocytogenes and help uncover yet unknown regulatory pathways. 


\section{Outlook}

We could show that the two most abundant cyclases CdaA and DisA are both regulated by osmoregulatory mechanisms, namely cellular turgor (CdaA with $\mathrm{CdaR}$ ) and molecular crowding and DNA-ion interaction (DisA). We furthermore could show potential cluster building of synthesis and degradation machinery in L. monocytogenes that will be interesting subjects for further studies. As summarized in Fig. 7.2, L. monocytogenes possesses a great repertoire of osmolyte uptake and efflux systems.
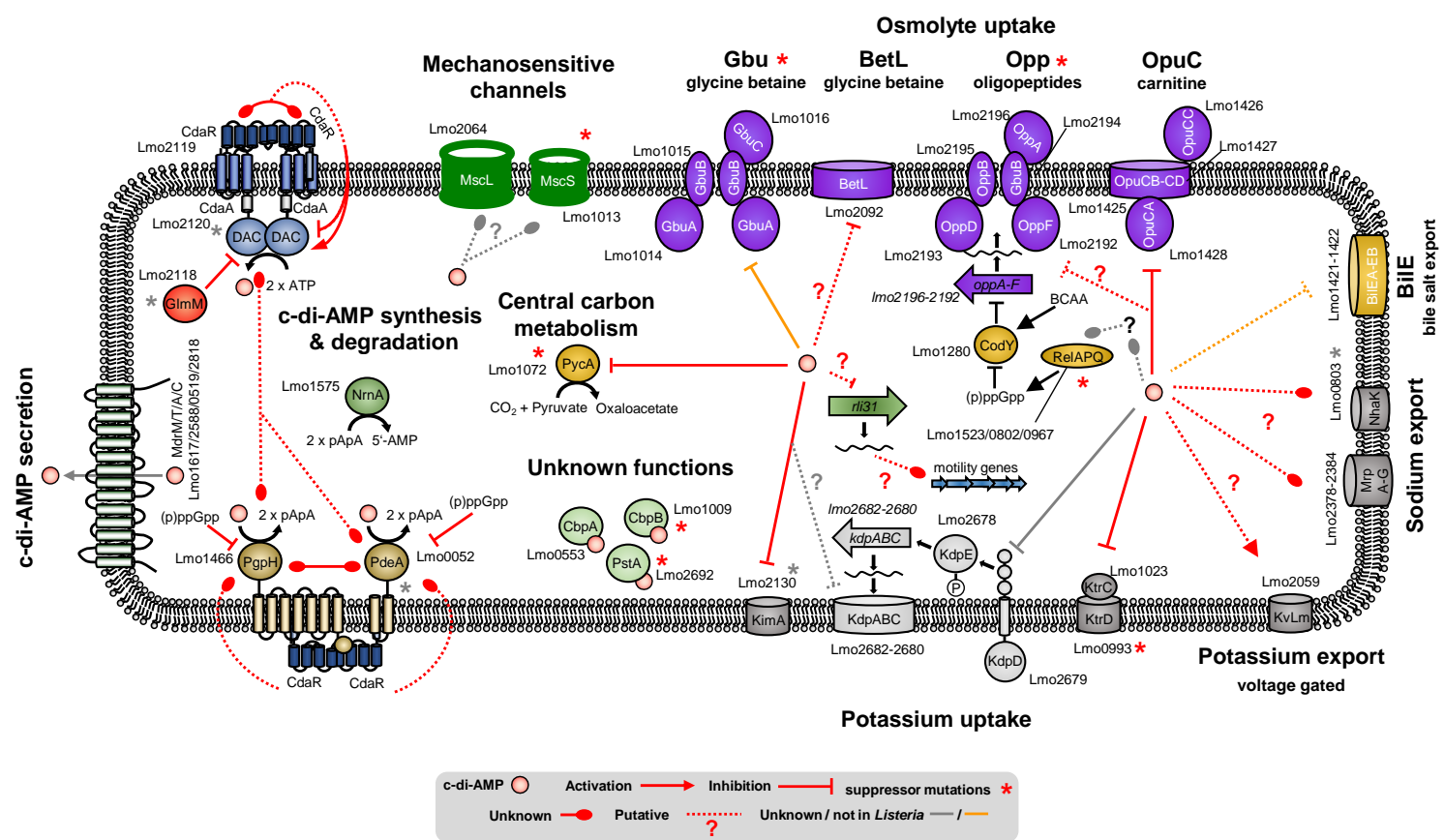

Fig. 7.2 An outlook on c-di-AMP signaling and osmoregulation in L. monocytogenes. In addition to what is discussed and shown in Fig. 7.1, c-di-AMP might affect other osmoregulatory systems in L. monocytogenes that have been connected to c-di-AMP metabolism and or osmoregulation in other organisms or that would make sense to be regulated by c-di-AMP as the major regulator of osmotic homeostasis in Firmicutes. L. monocytogenes possesses two mechanosensitive channels that may be a fitting target for the osmoregulator c-di-AMP. It could, furthermore, regulate glycine betaine transport by Gbu, despite the currently contradicting studies in L. monocytogenes and might also regulate uptake via the BetL system. Furthermore, regulation of the Opp oligopeptide importers is tightly linked to c-di-AMP via the cross-talk with the stringent response and frequent occurrence of suppressor mutations and suggests therefore the possibility of a more direct influence of c-di-AMP on Opp activity. It would, moreover, be interesting to study if c-di-AMP has an effect on BilE activity, despite studies showing no binding so far and to see if it impacts potassium and sodium efflux systems that would physiologically make sense to be c-di-AMP regulated. In future studies it would, furthermore, be interesting to analyze the cross-talk between the stringent response and c-di-AMP pathways. Moreover, it will be interesting to see if $\mathrm{KdpD}$ in L. monocytogenes also regulate $k d p A B C$ expression in dependency of c-di-AMP and if c-di-AMP, as it is the case for other potassium uptake systems, controls KdpABC activity directly. Lastly, the elucidation of the function of yet unknown proteins like $\mathrm{CbpA}, \mathrm{CbpB}$ and $\mathrm{Pst} A$ and the regulation of degradation, synthesis and the potential cross-talk between those two interesting determinants of the intracellular c-di-AMP concentration can be important aspects of future studies of this fascination nucleotide second messenger (modified from Commichau et al., 2018).

It will be interesting in the future to elucidate if other osmoregulatory system in L. monocytogenes or related bacteria are also regulated by c-di-AMP. Regulation could hereby occur on all levels from transcription, over translation, to control of protein activity. Possible targets for such a regulation are yet not studied osmolyte systems, mechanosensitive channels, osmolyte export systems and proteins of other signaling pathways that are non to interfere with c-di-AMP signaling. Analysis of c-di-AMP dependent expression could be analyzed by promoter-reporter gene fusion 
experiments. For the analysis of c-di-AMP protein interactions, DRaCALAs can be performed (see chapter 3). Another important subject will be the study of the Kdp potassium transporter in L. monocytogenes. In S. aureus it is known that its expression is regulated by the KdpDE two-component system, which is also present in L. monocytogenes. Furthermore, given that the Kdp system is also a high-affinity potassium importer, it would make sense to have a two-level regulation, meaning also regulation of the transporter activity, by c-di-AMP, as it is known for KtrAB in $B$. subtilis. The Kdp system could be investigated, similar to the characterization of KtrCD and KimA potassium transporters (see chapter 3 ). It will be also interesting to study the role of the c-di-AMP binding proteins of yet unknown function ( $\mathrm{CbpA}, \mathrm{CbpB}$ and $\mathrm{Pst} A)$ and their regulation by c-di-AMP. Moreover, identifying novel pathways or processes that might be regulated by c-di-AMP will enhance the knowledge about nucleotide signaling and possible open novel research possibilities. The data about differentially expressed genes and c-di-AMP influenced protein abundancy will be a great starting point to elucidate possible new regulatory pathways. As demonstrated in chapter 4 and discussed above, regulation of motility gene expression might already be a new c-di-AMP regulated pathway in L. monocytogenes, with possible implications of the sRNA rli31 (chapter 6).

The most interesting will be the studies of synthesis and degradation of c-di-AMP and the regulation by osmotic changes and possible cross-talk. The next important experiments should be the analysis of the influence of $\mathrm{CdaR}$ on CdaA self-interaction and determination if CdaA-CdaR interaction is transient and subject to regulation or consecutive. Possible experiments to analyze this, are the expression of CdaA-split Gfp fusion proteins or $\mathrm{CdaA} / \mathrm{CdaR}$ fluorophore fusion proteins to analyze CdaA self-interaction or CdaA-CdaR co-localization, respectively. Evaluation of c-di-AMP synthesis in mutants with an increased or decreased cell wall structure, by differential expression of cell wall-metabolic enzymes or the study of c-di-AMP synthesis in cell wall-less bacteria (Lforms) could further help to determine the exact mode of action CdaR has on CdaA activity. For analysis of $\mathrm{CdaA}$ regulation by $\mathrm{GImM}$, in vitro and in vivo studies should be conducted to compare effects of GImM from L. monocytogenes and GImM of non-c-di-AMP synthesizing bacteria, such as $E$. coli on the c-di-AMP concentration.

As discussed above, we could increase the knowledge of DAC regulation and propose novel hypothesis for regulation of DAC activity and a potential interaction between synthesis and degradation machineries. It will be very interesting to study those regulatory mechanisms further in the future. This might ultimately also lead to the discovery of novel antibiotics that target c-di-AMP signaling and thereby osmoregulation. Especially in combination with cell wall-weakening antibiotics of the $\beta$-lactam class, this could lead to novel and putatively effective combination treatments to fight the ever-increasing occurrence of multi-resistant pathogenic bacteria. 


\section{Supplementary Data}

\section{Chapter 2}

Tab. S2.1 Plasmids

\begin{tabular}{|c|c|c|}
\hline Name & Insert/Features & Reference \\
\hline pBAD33 & $\mathrm{P}_{\mathrm{BAD}-\mathrm{mcs} \text { araC cat }}$ & Guzman et al., 1995 \\
\hline pGP172 & $\mathrm{P}_{\mathrm{T} 7}-\mathrm{mcs}$ bla & Merzbacher et al., 2004 \\
\hline pIMK3 & $\mathrm{P}_{\text {help-laco-mcs lacl neo }}$ & Monk et al., 2008 \\
\hline pMAD & bla erm $C$ bgaB & Arnaud et al., 2004 \\
\hline pKTop & $\mathrm{P}_{\text {lac }}$-mcs-phoA $A_{66-1416}-I_{a c Z_{12-180}}$ aphA3 & Karimova et al., 2009 \\
\hline pUT18 & $\mathrm{P}_{l a c}-\mathrm{mcs}-c y a \mathrm{~T} 18$ bla & Karimova et al., 1998 \\
\hline pUT18C & $\mathrm{P}_{l a c}-c y a \mathrm{~T} 18-\mathrm{mcs} b / a$ & Karimova et al., 1998 \\
\hline pUT18C-zip & $\mathrm{P}_{\text {lac }}$-cyaT18-yeast GCN4 leucine zipper bla & Karimova et al., 1998 \\
\hline $\mathrm{p} 25-\mathrm{N}$ & $\mathrm{P}_{\text {lac }}$-mcs-cyaT25 aphA3 & Claessen et al., 2008 \\
\hline pKT25 & $\mathrm{P}_{\text {lac }}$-cyaT25-mcs aphA3 & Karimova et al., 1998 \\
\hline pKT25-zip & 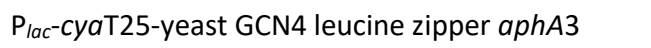 & Karimova et al., 1998 \\
\hline pBP131 & pGP172-prfA (Imo0200) & This work \\
\hline pBP223 & pGP172-cdaR ( $\triangle$ aa 1-28) & This work \\
\hline pBP224 & pUT18-cdaR (Imo2119) & Rismondo et al., 2016 \\
\hline pBP225 & pUT18C-cdaR & Rismondo et al., 2016 \\
\hline pBP226 & $\mathrm{p} 25-\mathrm{N}-c d a R$ & Rismondo et al., 2016 \\
\hline pBP227 & pKT25-cdaR & Rismondo et al., 2016 \\
\hline pBP232 & pUT18-cdaA (Imo2120) & Rismondo et al., 2016 \\
\hline pBP233 & pUT18C-cdaA & Rismondo et al., 2016 \\
\hline pBP234 & $\mathrm{p} 25-\mathrm{N}-c d a A$ & Rismondo et al., 2016 \\
\hline pBP235 & pKT25-cdaA & Rismondo et al., 2016 \\
\hline pBP250 & pKTop-cdaR & This work \\
\hline pBP251 & pKTop-cdaR (aa 1-33; $\Delta$ YbbR domains) & This work \\
\hline pBP252 & pKTop-cdaR (aa 34-452; $\Delta \mathrm{TM}$ domain) & This work \\
\hline pBP253 & pKTop-prkA (Imo1820) & This work \\
\hline pBP254 & pKTop-prfA & This work \\
\hline pBP255 & pIMK3-cdaR (aa 32-452; $\Delta$ TM domain) & This work \\
\hline pBP256 & pIMK3-cdaR (aa 1-320; $\Delta$ YbbR domain 4) & This work \\
\hline pBP257 & pIMK3-cdaR (aa 1-230; $\Delta$ YbbR domain 3-4) & This work \\
\hline pBP258 & pIMK3-cdaR (aa 1-130; $\Delta$ YbbR domain 2-4) & This work \\
\hline pBP259 & pIMK3-cdaR (aa 1-33; $\Delta$ YbbR domain 1-4) & This work \\
\hline pBP260 & pBAD33-cdaA-cdaR (aa 34-452; $\triangle \mathrm{TM}$ domain) & This work \\
\hline pBP261 & pBAD33-cdaA-cdaR (aa 1-320; $\Delta \mathrm{YbbR}$ domain 4) & This work \\
\hline pBP262 & pBAD33-cdaA-cdaR (aa 1-230; $\Delta \mathrm{YbbR}$ domain 3-4) & This work \\
\hline pBP263 & pBAD33-cdaA-cdaR (aa 1-130; $\Delta \mathrm{YbbR}$ domain 2-4) & This work \\
\hline pBP264 & pBAD33-cdaA-cdaR (aa 1-33; $\Delta$ YbbR domain 1-4) & This work \\
\hline pBP269 & pUT18-pdeA (Imo0052) & This work \\
\hline pBP270 & pUT18C-pdeA & This work \\
\hline pBP271 & p25-N-pdeA & This work \\
\hline pBP272 & pKT25-pdeA & This work \\
\hline pBP273 & pUT18-pgpH (Imo1466) & This work \\
\hline
\end{tabular}


Cyclic di-AMP and osmoregulation in Listeria monocytogenes

\begin{tabular}{|c|c|c|}
\hline Name & Insert/Features & Reference \\
\hline pBP274 & pUT18C-pgpH & This work \\
\hline pBP275 & $\mathrm{p} 25-\mathrm{N}-\mathrm{pgpH}$ & This work \\
\hline pBP276 & pKT25-pgpH & This work \\
\hline pBP277 & pUT18-kimA (Imo2130) & This work \\
\hline pBP278 & pUT18C-kimA & This work \\
\hline pBP279 & p25-N-kimA & This work \\
\hline pBP280 & pKT25-kimA & This work \\
\hline pBP352 & pMAD- $\triangle c d a A$ ( $c d a A$ up- and downstream region) & This work \\
\hline pBP359 & pUT18-g/mM (Imo2118) & This work \\
\hline pBP360 & pUT18C-glmM & This work \\
\hline pBP361 & p25-N-glmM & This work \\
\hline pBP362 & pKT25-glmM & This work \\
\hline pBP370 & pBAD33-cdaA & Quintana et al., 2019 \\
\hline pBP373 & pBAD33-cdaA D171N & Quintana et al., 2019 \\
\hline pBP384 & pWH844-kimA & Chapter 3 \\
\hline pBP387 & pBAD33-cdaA-cdaR & This work \\
\hline pBP388 & pBP387-g/mM & This work \\
\hline pBP389 & pBP370-g/mM & This work \\
\hline pGP976 & pUT18-rnYBsu & Commichau et al., 2009 \\
\hline pGP977 & pUT18C-rnYBsu & Commichau et al., 2009 \\
\hline pGP978 & $\mathrm{p} 25-\mathrm{N}-r n Y^{B s u}$ & Commichau et al., 2009 \\
\hline pGP979 & pKT25-rnYBsu & Commichau et al., 2009 \\
\hline
\end{tabular}

Tab. S2.2 Oligonucleotides

\begin{tabular}{|c|c|c|}
\hline Name & $\begin{array}{l}\text { Restriction sites are underlined, complementary regions are in bold, se- } \\
\text { quences } 5^{\prime} \rightarrow 3^{\prime}\end{array}$ & Purpose and reference \\
\hline FC205 & TTTAGATCTTTAATTTAATTTTCCCCAAGTAGCAGGACATGC & Rev. prfA (Bg/lI) \\
\hline FC206 & AAAGAGCTCGATGAACGCTCAAGCAGAAGAATTCAAA & Fwd. prfA (Sacl) \\
\hline FC336 & AAATCTAGAGATGGGTAAATATTTCGGTACGGATGGAGTTAG & Fwd. $g \operatorname{lmM}(X b a l)$ \\
\hline FC337 & TTTGGTACCCGATCGTTAAGTGCCATTTCTGAACGAACAACCG & Rev. $g$ ImM (Kpnl) \\
\hline JH05 & AAAGAATTCAGAATTGCGTTCCACGGATACATTAAAAC & $\begin{array}{l}\text { Fwd. } c d a A \text { upstream re- } \\
\text { gion (EcoRI) }\end{array}$ \\
\hline JH06 & CCTCCTTTCGTCGACGTGCCTCTTGAAAACCATTTATAATCAC & $\begin{array}{l}\text { Rev. } c d a A \text { upstream re- } \\
\text { gion }(S a c l)\end{array}$ \\
\hline $\mathrm{JH} 07$ & AAGAGGCACGTCGACGAAAGGAGGCAAAAGCGAATGATG & $\begin{array}{l}\text { Fwd. cdaA downstream } \\
\text { region (Sacl) }\end{array}$ \\
\hline JH08 & TTTGGATCCCACTTTCCGGCGTGCCTTCTTG & $\begin{array}{l}\text { Rev. } c d a A \text { downstream } \\
\text { region (BamHI) }\end{array}$ \\
\hline $\mathrm{JH} 21$ & AAACCATGGATCGAATTTTAAATAATAAATGGTCGATTC & $\begin{array}{l}\text { Fwd. cdaR (Ncol), Ris- } \\
\text { mondo et al., } 2016\end{array}$ \\
\hline $\mathrm{JH} 22$ & TTTGTCGACTTATGTGCTTTTGGAAGGTACTTCAATGG & $\begin{array}{l}\text { Rev. cdaR (Sall), Ris- } \\
\text { mondo et al., } 2016\end{array}$ \\
\hline JH51 & AAATCTAGACACGGAGGTGAAGTGATGGATTTTTCCAATATGTCGATATTGCAT & $\begin{array}{l}\text { Fwd. cdaA (Xbal), Quin- } \\
\text { tana et al., } 2019\end{array}$ \\
\hline $\mathrm{JH} 103$ & TTTCTGCAGTTATGTGCTTTTGGAAGGTACTTCAATGGATG & Rev. cdaR (Pstl) \\
\hline $\mathrm{JH} 104$ & AAACTGCAGAGAAGGAGAGTAATGAAATGGGTAAATATTTCG & Fwd. $g / m M(P s t \mid)$ \\
\hline
\end{tabular}




\begin{tabular}{|c|c|c|}
\hline Name & $\begin{array}{l}\text { Restriction sites are underlined, complementary regions are in bold, se- } \\
\text { quences } 5^{\prime} \rightarrow 3^{\prime}\end{array}$ & Purpose and reference \\
\hline $\mathrm{JH} 105$ & TTTAAGCTTTTAATCGTTAAGTGCCATTTCTGAACGAACAAC & Rev. glmM (HindIII) \\
\hline JH121 & AAAGGATCCCATGGATCGAATTTTAAATAATAAATGGTCGATTCGAAT & Fwd. $c d a R(B a m H I)$ \\
\hline JH122 & TTTGGTACCGCTGTGCTTTTGGAAGGTACTTCAATGGATGC & Rev. cdaR (Kpnl) \\
\hline JH123 & AAAGGATCCCATGACGACTTTTTTCTACGACGTCTTCTAGTGATTC & $\begin{array}{l}\text { Fwd. cdaR ( } \triangle \text { aa 1-33; } \\
\text { BamHI) }\end{array}$ \\
\hline JH124 & TTTGGTACCGCGGCGTTATTATTATTTGCATTAACTGATGTAAAAAGG & $\begin{array}{l}\text { Rev. cdaR ( } \triangle \text { aa 34-452; } \\
\text { Kpnl) }\end{array}$ \\
\hline JH126 & AAAGGATCCCATGATGATTGGTAAGCGATTAAGCGATCG & Fwd. prkA (BamHI) \\
\hline $\mathrm{JH} 127$ & TTTGGTACCGCATTTGGATAAGGGACTGTACCTTCATCG & Rev. prkA (Kpnl) \\
\hline $\mathrm{JH} 128$ & AAATCTAGACATGAACGCTCAAGCAGAAGAATTCAAAAAATATTTAG & Fwd. prfA (Xbal) \\
\hline JH129 & TTTGGTACCGCATTTAATTTTCCCCAAGTAGCAGGACATGC & Rev. prfA (Kpnl) \\
\hline $\mathrm{JH} 130$ & AAACCATGGCCACGACTTTTTTCTACGACGTCTTCTAG & $\begin{array}{l}\text { Fwd. cdaR ( } \triangle \text { aa 1-31; } \\
\text { Ncol) }\end{array}$ \\
\hline JH131 & TTTGTCGACTTAGGCGTTATTATTATTTGCATTAACTGATGTAAAAAGG & $\begin{array}{l}\text { Rev. cdaR ( } \triangle \text { aa 34-452; } \\
\text { Sall) }\end{array}$ \\
\hline JH132 & TTTGTCGACTTAGTTATTTGCTTCAGACTTTTTTACGGTCTTAATC & $\begin{array}{l}\text { Rev. cdaR ( } \triangle \text { aa 321-452; } \\
\text { Sall) }\end{array}$ \\
\hline JH133 & TTTGTCGACTTACTTGCCGACTTTTTCAACTGGCACG & $\begin{array}{l}\text { Rev. cdaR ( } \triangle \text { aa 231-452; } \\
\text { Sall) }\end{array}$ \\
\hline $\mathrm{JH} 134$ & TTTGTCGACTTATTCTTGTACATTTACGTTGACTGTTGCTGGATTC & $\begin{array}{l}\text { Rev. cdaR ( } \triangle \text { aa 131-452; } \\
\text { Sall) }\end{array}$ \\
\hline JH135 & CGTAGAAAAAGTCGTCATCATTCGCTTTTTGCCTCCTTTCCATTTAG & Rev. cdaA \\
\hline JH136 & CAAAAGCGAATGATGACGACTTTTTCTACGACGTCTTCTAGTGATTC & Fwd. cdaR ( $\triangle$ aa 1-33) \\
\hline $\mathrm{JH} 137$ & TTTCTGCAGTTAGGCGTTATTATTATTTGCATTAACTGATGTAAAAAGG & $\begin{array}{l}\text { Rev. cdaR ( } \triangle \text { aa 34-452; } \\
\text { Pstl) }\end{array}$ \\
\hline $\mathrm{JH} 138$ & TTTCTGCAGTTAGTTATTTGCTTCAGACTTTTTTACGGTCTTAATC & $\begin{array}{l}\text { Rev. cdaR ( } \triangle \text { aa 321-452; } \\
\text { Pstl) }\end{array}$ \\
\hline JH139 & TTTCTGCAGTTACTTGCCGACTTTTTCAACTGGCACG & $\begin{array}{l}\text { Rev. cdaR ( } \triangle \text { aa 231-452; } \\
\text { Pstl) }\end{array}$ \\
\hline $\mathrm{JH} 140$ & TTTCTGCAGTTATTCTTGTACATTTACGTTGACTGTTGCTGGATTC & $\begin{array}{l}\text { Rev. cdaR ( } \triangle \text { aa 131-452; } \\
\text { Pstl) }\end{array}$ \\
\hline $\mathrm{JH} 160$ & AAATCTAGAGATGTCAGGCTATTTTCAAAAACGAATGCTTAAATATCC & Fwd. pdeA (Xbal) \\
\hline JH161 & TTTGGTACCCGTGTTTCTCCCTTCCAATACGCATCAATGG & Rev. pdeA (Kpnl) \\
\hline JH162 & AAATCTAGAGGTGAAACTAGCCAAGAAATGGAGAGATTGG & Fwd. $p g p H(X b a l)$ \\
\hline JH163 & TTTGGTACCCGATCTTTGTCATCAGGATATTGAATTCGTTGATGATATATTC & Rev. $p g p H(K p n l)$ \\
\hline JH164 & AAATCTAGAGATGGCTTCGCCGCTAAAAAGACTATTAATCG & Fwd. $\operatorname{kimA}(X b a l)$ \\
\hline JH165 & TTTGGTACCCGTTCTTTTAAATGATAAGGATATGTGGAAACTACTACATCC & Rev. $\operatorname{kimA}(K p n l)$ \\
\hline JR28 & TTTGGATCCTTATGTGCTTTTGGAAGGTAC & Rev. cdaR (BamHI) \\
\hline JR56 & AAAGAGCTCGAATAATAATAACGCCACGACTTTTTTCTACG & $\begin{array}{l}\text { Fwd. cdaR ( } \triangle \text { aa 1-28; } \\
\text { Sacl) }\end{array}$ \\
\hline
\end{tabular}




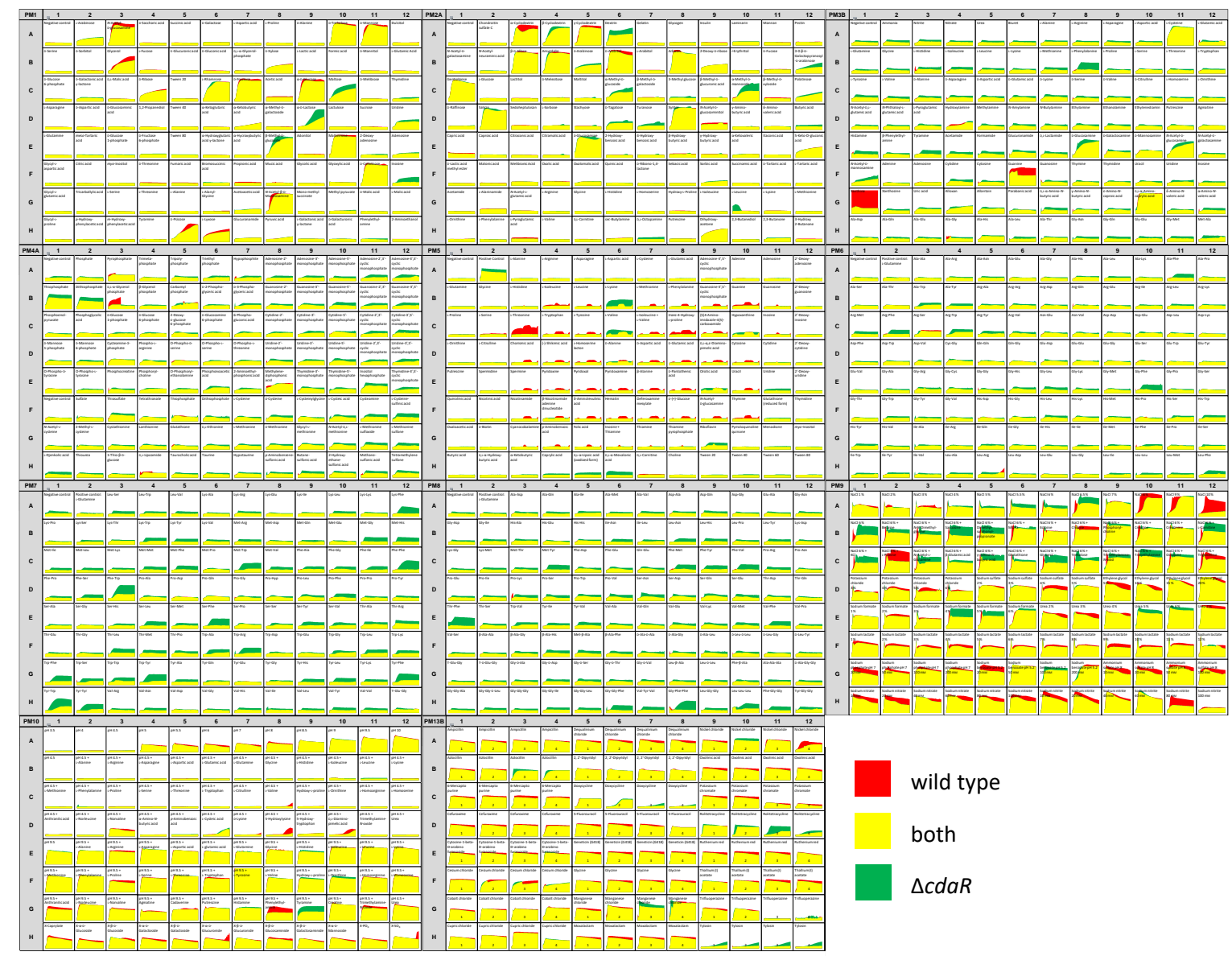

Fig. S2.1 Biolog Phenotype Microarray (PM). Images of plate PM1-10 and PM13B. Metabolic activity of the wild type is shown in red and of the $\triangle c d a R$ mutant in green. Overlapping regions between them are shown in yellow.

\section{Chapter 4}

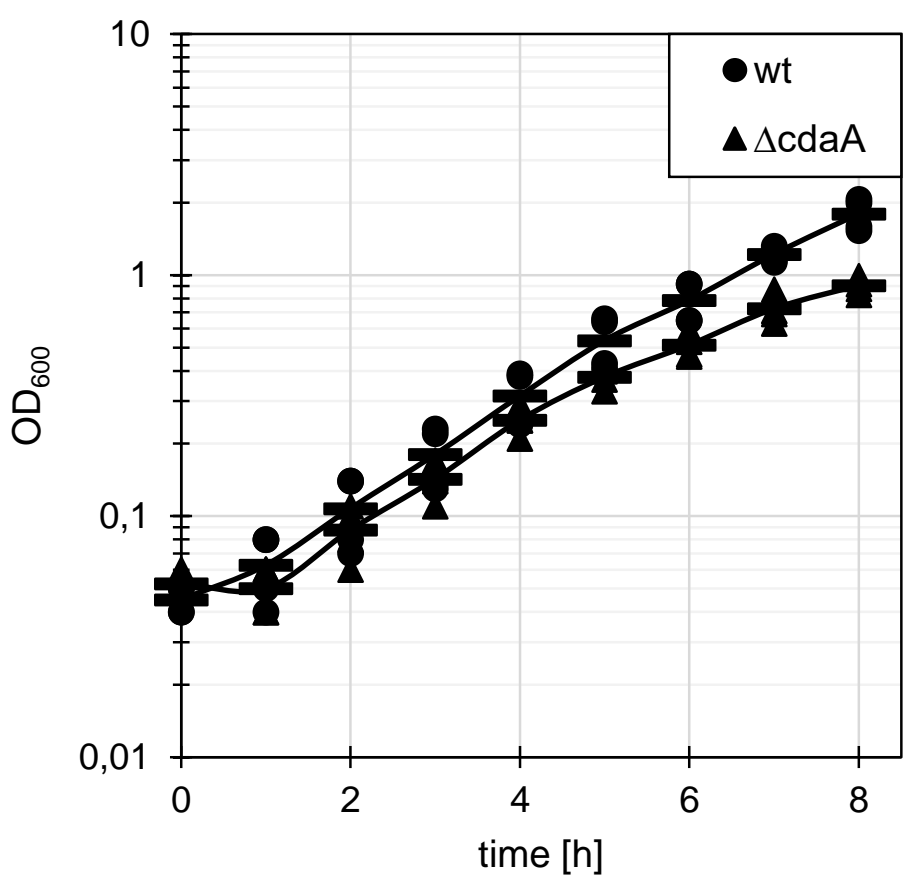

Fig. S4.1 The cdaA deletion strain grows similar to the wild type in LSM. The L. monocytogenes wt and the $\triangle c d a A$ mutant (BPL77) were cultivated overnight in $5 \mathrm{ml} \mathrm{LSM}$ at $37^{\circ} \mathrm{C}$ and $220 \mathrm{rpm}$ from single colonies. Preculture were used to inoculate $10 \mathrm{ml} \mathrm{LSM}$ to an $\mathrm{OD}_{600}$ of 0.05 and cultures incubated at $37^{\circ} \mathrm{C}$ and $220 \mathrm{rpm}$. The $\mathrm{OD}_{600}$ was measured hourly. Shown are measurements of four biological replicates and the mean $\mathrm{OD}_{600}$ values as horizontal lines. 
Tab. S4.1 Complete list of differentially expressed genes or synthesized proteins in $\Delta c d a A$ vs. the wt

\begin{tabular}{|c|c|c|c|c|c|c|c|c|c|}
\hline \multirow{2}{*}{ Locus tag } & \multirow{2}{*}{ Operon } & \multirow{2}{*}{ Name } & \multirow{2}{*}{ RAST info } & \multirow{2}{*}{ Function (COG) } & \multicolumn{2}{|c|}{$\begin{array}{c}\text { Transcriptome } \\
\Delta c d a A / w t\end{array}$} & \multicolumn{3}{|c|}{$\begin{array}{l}\text { Proteome } \\
\Delta c d a A / w t\end{array}$} \\
\hline & & & & & $\begin{array}{c}\text { Fold } \\
\text { change }\end{array}$ & $\mathbf{p}$ & $\begin{array}{c}\text { Fold } \\
\text { change }\end{array}$ & $\mathbf{p}$ & $p, f d r$ \\
\hline Imo0118 & 20 & $\operatorname{ImaA}$ & hypothetical protein & Function unknown & & & -1.32 & $0.0 \mathrm{E}+00$ & $6.0 \mathrm{E}-04$ \\
\hline Imo0119 & 20 & & $\begin{array}{l}\text { FIG00774706: hypothetical pro- } \\
\text { tein }\end{array}$ & Not in COGs & & & -1.46 & $1.0 \mathrm{E}-04$ & $1.6 \mathrm{E}-03$ \\
\hline Imo0186 & & & Cell wall-binding protein & Function unknown & -10.07 & $0.0 \mathrm{E}+00$ & & & \\
\hline Imo0201 & 32 & plcA & $\begin{array}{l}\text { Phosphatidylinositol-specific } \\
\text { phospholipase C }\end{array}$ & $\begin{array}{l}\text { Defense/virulence mecha- } \\
\text { nisms }\end{array}$ & & & 1.87 & $0.0 \mathrm{E}+00$ & $0.0 E+00$ \\
\hline Imo0286 & & ykrV & $\begin{array}{l}\text { Glutamine-dependent 2-keto- } \\
\text { 4-methylthiobutyrate transam- } \\
\text { inase }\end{array}$ & $\begin{array}{l}\text { Amino acid transport and } \\
\text { metabolism }\end{array}$ & -2.37 & $4.9 \mathrm{E}-03$ & & & \\
\hline $\begin{array}{l}\text { Imo0557 } \\
\text { Imo0559 }\end{array}$ & 90 & & $\begin{array}{l}\text { Phosphoglycerate mutase fam- } \\
\text { ily } 1 \\
\text { Magnesium and cobalt } \\
\text { transport protein CorA }\end{array}$ & $\begin{array}{l}\text { Carbohydrate transport } \\
\text { and metabolism } \\
\text { Inorganic ion transport } \\
\text { and metabolism }\end{array}$ & 6.6 & $2.5 \mathrm{E}-03$ & 1.22 & $0.0 \mathrm{E}+00$ & $0.0 \mathrm{E}+00$ \\
\hline Imo0560 & & rocG & $\begin{array}{l}\text { NADP-specific glutamate dehy- } \\
\text { drogenase }\end{array}$ & $\begin{array}{l}\text { Amino acid transport and } \\
\text { metabolism }\end{array}$ & 2.46 & $3.0 \mathrm{E}-04$ & 1.18 & $0.0 \mathrm{E}+00$ & $0.0 \mathrm{E}+00$ \\
\hline Imo0647 & & & $\begin{array}{l}\text { FIG00774323: hypothetical pro- } \\
\text { tein }\end{array}$ & Not in COGs & 5.21 & $5.0 \mathrm{E}-04$ & & & \\
\hline Imo0683 & 111 & cheR & $\begin{array}{l}\text { Chemotaxis protein methyl- } \\
\text { transferase CheR }\end{array}$ & $\begin{array}{l}\text { Cell motility; Signal trans- } \\
\text { duction mechanisms; }\end{array}$ & 2.68 & $5.4 \mathrm{E}-03$ & & & \\
\hline Imo0684 & 111 & & $\begin{array}{l}\text { FIG00774517: hypothetical pro- } \\
\text { tein }\end{array}$ & Not in COGs & 2.08 & $4.1 \mathrm{E}-03$ & & & \\
\hline Imo0687 & 111 & & $\begin{array}{l}\text { FIG00774408: hypothetical pro- } \\
\text { tein }\end{array}$ & Not in COGs & 2.17 & $6.5 \mathrm{E}-03$ & & & \\
\hline Imo0689 & 111 & cheV & Chemotaxis protein CheV & $\begin{array}{l}\text { Cell motility; Signal trans- } \\
\text { duction mechanisms; }\end{array}$ & & & 2.17 & $0.0 \mathrm{E}+00$ & $0.0 E+00$ \\
\hline Imo0694 & 112 & & $\begin{array}{l}\text { FIG00774728: hypothetical pro- } \\
\text { tein }\end{array}$ & Not in COGs & 7.44 & $2.2 \mathrm{E}-03$ & & & \\
\hline Imo0695 & 112 & & hypothetical protein & Cell motility & 8.66 & $3.0 \mathrm{E}-04$ & & & \\
\hline Imo0696 & 112 & flgD & $\begin{array}{l}\text { Flagellar basal-body rod modifi- } \\
\text { cation protein FlgD }\end{array}$ & Cell motility & 8.11 & $1.0 \mathrm{E}-04$ & & & \\
\hline Imo0697 & 112 & flgE & Flagellar hook protein FlgE & Cell motility & 6.99 & $3.0 \mathrm{E}-04$ & 3.43 & $7.0 \mathrm{E}-04$ & $8.2 \mathrm{E}-03$ \\
\hline Imo0698 & 112 & & $\begin{array}{l}\text { Flagellar motor switch protein } \\
\text { FliN }\end{array}$ & $\begin{array}{l}\text { Cell motility; Intracellular } \\
\text { trafficking and secretion; }\end{array}$ & 6.46 & $0.0 \mathrm{E}+00$ & & & \\
\hline Imo0699 & 112 & flim & $\begin{array}{l}\text { Flagellar motor switch protein } \\
\text { FliM }\end{array}$ & Cell motility & 6.69 & 4.0E-04 & & & \\
\hline Imo0700 & 112 & & $\begin{array}{l}\text { Flagellar motor switch protein } \\
\text { FliN }\end{array}$ & $\begin{array}{l}\text { Cell motility; Intracellular } \\
\text { trafficking and secretion; } \\
\text { Signal transduction mech- } \\
\text { anisms; }\end{array}$ & 5.03 & $2.0 \mathrm{E}-03$ & & & \\
\hline Imo0701 & 112 & & $\begin{array}{l}\text { FIG00774560: hypothetical pro- } \\
\text { tein }\end{array}$ & Not in COGs & 5.52 & 4.0E-03 & & & \\
\hline Imo0702 & 112 & & $\begin{array}{l}\text { FIG00774477: hypothetical pro- } \\
\text { tein } \\
\text { UDP-N-acetylenolpyruvoylglu- }\end{array}$ & Function unknown & 5.38 & $5.7 \mathrm{E}-03$ & & & \\
\hline Imo0703 & 112 & & $\begin{array}{lll}\text { cosamine } & \text { reductase } & \text { (EC } \\
\text { 1.1.1.158) } & & \end{array}$ & Not in COGs & 4.8 & 4.0E-04 & & & \\
\hline Imo0704 & 112 & & $\begin{array}{l}\text { FIG00774686: hypothetical pro- } \\
\text { tein }\end{array}$ & Not in COGs & 4.75 & $1.5 \mathrm{E}-03$ & 1.05 & $2.0 \mathrm{E}-04$ & $3.6 \mathrm{E}-03$ \\
\hline Imo0705 & 112 & flgK & $\begin{array}{l}\text { Flagellar hook-associated pro- } \\
\text { tein FlgK }\end{array}$ & Cell motility & 5.15 & $1.0 \mathrm{E}-04$ & & & \\
\hline Imo0706 & 112 & flgL & $\begin{array}{l}\text { Flagellar hook-associated pro- } \\
\text { tein FlgL }\end{array}$ & Cell motility & 5.25 & $0.0 \mathrm{E}+00$ & & & \\
\hline Imo0708 & 112 & flis & $\begin{array}{l}\text { Flagellar biosynthesis protein } \\
\text { Flis }\end{array}$ & $\begin{array}{l}\text { Cell motility; Posttransla- } \\
\text { tional modification, pro- } \\
\text { tein turnover, chaperones; } \\
\text { Intracellular trafficking } \\
\text { and secretion; }\end{array}$ & 4.46 & $1.0 \mathrm{E}-04$ & 7.45 & $1.0 \mathrm{E}-04$ & $1.0 \mathrm{E}-03$ \\
\hline Imo0709 & 112 & & $\begin{array}{l}\text { FIG00774899: hypothetical pro- } \\
\text { tein }\end{array}$ & & 4.83 & $3.0 \mathrm{E}-04$ & & & \\
\hline Imo0710 & 112 & flgB & $\begin{array}{l}\text { Flagellar basal-body rod protein } \\
\text { FlgB }\end{array}$ & Cell motility & 4.71 & $2.0 \mathrm{E}-04$ & & & \\
\hline Imo0711 & 112 & flgC & $\begin{array}{l}\text { Flagellar basal-body rod protein } \\
\text { FlgC }\end{array}$ & Cell motility & 4.25 & $8.0 \mathrm{E}-04$ & & & \\
\hline Imo0712 & 112 & fliE & $\begin{array}{l}\text { Flagellar hook-basal body com- } \\
\text { plex protein FliE }\end{array}$ & $\begin{array}{l}\text { Cell motility; Intracellular } \\
\text { trafficking and secretion; }\end{array}$ & 4.41 & $9.0 \mathrm{E}-04$ & & & \\
\hline
\end{tabular}




\begin{tabular}{|c|c|c|c|c|c|c|c|c|c|}
\hline \multirow{2}{*}{ Locus tag } & \multirow{2}{*}{ Operon } & \multirow{2}{*}{ Name } & \multirow{2}{*}{ RAST info } & \multirow{2}{*}{ Function (COG) } & \multicolumn{2}{|c|}{$\begin{array}{c}\text { Transcriptome } \\
\Delta c d a A / w t\end{array}$} & \multicolumn{3}{|c|}{$\begin{array}{l}\text { Proteome } \\
\Delta c d a A / w t\end{array}$} \\
\hline & & & & & $\begin{array}{c}\text { Fold } \\
\text { change }\end{array}$ & $\mathbf{p}$ & $\begin{array}{c}\text { Fold } \\
\text { change }\end{array}$ & p & $p, f d r$ \\
\hline Imo0713 & 112 & fliF & Flagellar M-ring protein FliF & $\begin{array}{l}\text { Cell motility; Intracellular } \\
\text { trafficking and secretion; }\end{array}$ & 4 & $1.0 \mathrm{E}-02$ & 1.63 & $1.0 \mathrm{E}-04$ & $1.0 \mathrm{E}-03$ \\
\hline Imo0714 & 112 & fliG & $\begin{array}{l}\text { Flagellar motor switch protein } \\
\text { FliG }\end{array}$ & Cell motility & & & 1.37 & $6.0 \mathrm{E}-04$ & $6.9 \mathrm{E}-03$ \\
\hline Imo0715 & 112 & fliH & Flagellar assembly protein FliH & Not in COGs & 3.94 & $9.7 \mathrm{E}-03$ & & & \\
\hline Imo0717 & 112 & yjbJ & $\begin{array}{l}\text { Soluble lytic murein transglyco- } \\
\text { sylase precursor }\end{array}$ & $\begin{array}{l}\text { Cell wall/membrane bio- } \\
\text { genesis }\end{array}$ & 3.68 & 4.7E-03 & & & \\
\hline Imo0718 & 112 & & $\begin{array}{l}\text { FIG00774152: hypothetical pro- } \\
\text { tein }\end{array}$ & Not in COGs & 3.75 & $1.0 \mathrm{E}-03$ & 1.01 & $1.0 \mathrm{E}-04$ & $1.9 \mathrm{E}-03$ \\
\hline Imo0723 & 114 & & $\begin{array}{l}\text { methyl-accepting chemotaxis } \\
\text { protein }\end{array}$ & $\begin{array}{l}\text { Cell motility; Signal trans- } \\
\text { duction mechanisms; }\end{array}$ & & & 1.18 & $0.0 \mathrm{E}+00$ & $0.0 \mathrm{E}+00$ \\
\hline Imo0809 & 127 & & $\begin{array}{l}\text { Spermidine Putrescine } A B C \\
\text { transporter permease compo- } \\
\text { nent potC }\end{array}$ & $\begin{array}{l}\text { Amino acid transport and } \\
\text { metabolism }\end{array}$ & -2.43 & $5.6 \mathrm{E}-03$ & & & \\
\hline Imo0810 & 127 & & $\begin{array}{l}A B C \text { transporter, periplasmic } \\
\text { spermidine putrescine-binding } \\
\text { protein PotD }\end{array}$ & $\begin{array}{l}\text { Amino acid transport and } \\
\text { metabolism }\end{array}$ & -2.61 & $1.3 \mathrm{E}-03$ & & & \\
\hline Imo0858 & & exuR & $\begin{array}{l}\text { DNA-binding transcriptional } \\
\text { regulator }\end{array}$ & Transcription & 2.28 & $2.8 \mathrm{E}-03$ & & & \\
\hline Imo0954 & 149 & & $\begin{array}{l}\text { FIG00774399: hypothetical pro- } \\
\text { tein }\end{array}$ & Not in COGs & 2.43 & $5.8 \mathrm{E}-03$ & & & \\
\hline Imo0971 & 155 & dltD & $\begin{array}{l}\text { Poly(glycerophosphate chain) } \\
\text { D-alanine transfer protein DltD }\end{array}$ & $\begin{array}{l}\text { Cell wall/membrane bio- } \\
\text { genesis }\end{array}$ & 3.9 & $6.1 \mathrm{E}-03$ & & & \\
\hline Imo0973 & 155 & dltB & D-alanyl transfer protein DltB & $\begin{array}{l}\text { Cell wall/membrane bio- } \\
\text { genesis }\end{array}$ & 5.78 & $3.3 \mathrm{E}-03$ & & & \\
\hline Imo0974 & 155 & dltA & $\begin{array}{l}\text { D-alanine--poly(phosphoribi- } \\
\text { tol) ligase subunit } 1\end{array}$ & $\begin{array}{l}\text { Cell wall/membrane bio- } \\
\text { genesis } \\
\text { Carbohydrate transport }\end{array}$ & 6.4 & $5.4 \mathrm{E}-03$ & 1.04 & $0.0 \mathrm{E}+00$ & $0.0 \mathrm{E}+00$ \\
\hline Imo1074 & 174 & tagG & $\begin{array}{l}\text { Teichoic acid translocation per- } \\
\text { mease protein TagG }\end{array}$ & $\begin{array}{l}\text { and metabolism; Cell } \\
\text { wall/membrane biogene- } \\
\text { sis; } \\
\text { Carbohydrate transport }\end{array}$ & -2.25 & $5.0 \mathrm{E}-04$ & & & \\
\hline Imo1075 & 174 & tagH & $\begin{array}{l}\text { Teichoic acid export ATP-bind- } \\
\text { ing protein TagH }\end{array}$ & $\begin{array}{l}\text { and metabolism; Cell } \\
\text { wall/membrane biogene- } \\
\text { sis; }\end{array}$ & -2.15 & $1.1 \mathrm{E}-03$ & & & \\
\hline Imo1126 & 182 & & acetyltransferase, GNAT family & $\begin{array}{l}\text { Transcription; General } \\
\text { function prediction only; }\end{array}$ & -2.23 & $1.6 \mathrm{E}-03$ & & & \\
\hline Imo1216 & & & $\begin{array}{l}\mathrm{N} \text {-acetylmuramoyl-L-alanine } \\
\text { amidase, family } 4\end{array}$ & $\begin{array}{l}\text { Cell motility; Intracellular } \\
\text { trafficking and secretion; }\end{array}$ & -17.85 & $7.0 \mathrm{E}-04$ & & & \\
\hline Imo1301 & 206 & & acetyltransferase, GNAT family & Translation & 2.01 & $7.0 \mathrm{E}-04$ & & & \\
\hline Imo1348 & 213 & gcvT & Aminomethyltransferase & $\begin{array}{l}\text { Amino acid transport and } \\
\text { metabolism }\end{array}$ & & & -1.06 & $0.0 \mathrm{E}+00$ & $0.0 \mathrm{E}+00$ \\
\hline Imo1389 & 224 & yufo & $\begin{array}{l}\text { Unspecified monosaccharide } \\
\text { ABC transport system }\end{array}$ & $\begin{array}{l}\text { General function predic- } \\
\text { tion only }\end{array}$ & -2.28 & $1.9 \mathrm{E}-03$ & & & \\
\hline Imo1390 & 224 & yufP & $\begin{array}{l}\text { Unspecified monosaccharide } \\
A B C \text { transport system }\end{array}$ & $\begin{array}{l}\text { General function predic- } \\
\text { tion only }\end{array}$ & -2.06 & $5.1 \mathrm{E}-03$ & & & \\
\hline Imo1433 & 235 & pdhD & Glutathione reductase & $\begin{array}{l}\text { Energy production and } \\
\text { conversion }\end{array}$ & & & 1.11 & $0.0 \mathrm{E}+00$ & $3.0 \mathrm{E}-04$ \\
\hline Imo1498 & 247 & yrrM & $\begin{array}{l}\text { FIG011945: O-methyltransfer- } \\
\text { ase family protein }\end{array}$ & $\begin{array}{l}\text { General function predic- } \\
\text { tion only }\end{array}$ & & & -1.18 & $1.0 \mathrm{E}-04$ & $1.0 \mathrm{E}-03$ \\
\hline Imo1566 & 265 & citC & $\begin{array}{l}\text { Isocitrate dehydrogenase } \\
\text { [NADP] }\end{array}$ & $\begin{array}{l}\text { Energy production and } \\
\text { conversion }\end{array}$ & & & 1.2 & $0.0 \mathrm{E}+00$ & $0.0 \mathrm{E}+00$ \\
\hline Imo1567 & 265 & citZ & Citrate synthase & $\begin{array}{l}\text { Energy production and } \\
\text { conversion }\end{array}$ & & & 1.12 & $0.0 \mathrm{E}+00$ & $0.0 \mathrm{E}+00$ \\
\hline Imo1597 & & & $\begin{array}{l}\text { FIG00774834: hypothetical pro- } \\
\text { tein }\end{array}$ & & 2.41 & $5.3 \mathrm{E}-03$ & & & \\
\hline Imo1636 & 281 & yhcH & $\mathrm{ABC}$ transporter & $\begin{array}{l}\text { Defense/virulence mecha- } \\
\text { nisms }\end{array}$ & 2.39 & $6.3 \mathrm{E}-03$ & & & \\
\hline Imo1637 & 281 & & $\begin{array}{l}A B C \text { transporter, permease } \\
\text { protein }\end{array}$ & $\begin{array}{l}\text { General function predic- } \\
\text { tion only }\end{array}$ & 2.55 & $1.6 \mathrm{E}-03$ & 1.04 & $0.0 \mathrm{E}+00$ & $0.0 \mathrm{E}+00$ \\
\hline Imo1641 & & citB & Aconitate hydratase & $\begin{array}{l}\text { Energy production and } \\
\text { conversion }\end{array}$ & 4.69 & $6.0 \mathrm{E}-04$ & 1.21 & $0.0 \mathrm{E}+00$ & $0.0 \mathrm{E}+00$ \\
\hline Imo1699 & 295 & & $\begin{array}{l}\text { methyl-accepting chemotaxis } \\
\text { protein }\end{array}$ & $\begin{array}{l}\text { Cell motility; Signal trans- } \\
\text { duction mechanisms; }\end{array}$ & 3.18 & $1.3 \mathrm{E}-03$ & & & \\
\hline Imo1700 & 295 & & $\begin{array}{l}\text { FIG00774912: hypothetical pro- } \\
\text { tein }\end{array}$ & Not in COGs & 4.53 & $1.9 \mathrm{E}-03$ & & & \\
\hline Imo1738 & 305 & artP & Amino acid $A B C$ transporter & $\begin{array}{l}\text { Amino acid transport and } \\
\text { metabolism; Signal trans- } \\
\text { duction mechanisms; }\end{array}$ & -2.38 & $9.0 \mathrm{E}-04$ & & & \\
\hline Imo1739 & 305 & artR & amino acid $A B C$ transporter & $\begin{array}{l}\text { Amino acid transport and } \\
\text { metabolism }\end{array}$ & -2.32 & $4.8 \mathrm{E}-03$ & & & \\
\hline Imo1751 & 307 & yefA & $\begin{array}{l}\text { 23S rRNA (Uracil-5-) -methyl- } \\
\text { transferase RumA }\end{array}$ & Translation & & & -1.77 & $1.0 \mathrm{E}-04$ & $2.0 \mathrm{E}-03$ \\
\hline Imo1764 & 310 & purD & $\begin{array}{l}\text { Phosphoribosylamine--glycine } \\
\text { ligase }\end{array}$ & $\begin{array}{l}\text { Nucleotide transport and } \\
\text { metabolism }\end{array}$ & -4.41 & $5.5 \mathrm{E}-03$ & & & \\
\hline Imo1765 & 310 & purH & $\begin{array}{l}\text { IMP cyclohydrolase/Phosphori- } \\
\text { bosylaminoimidazolecarbox- } \\
\text { amide formyltransferase }\end{array}$ & $\begin{array}{l}\text { Nucleotide transport and } \\
\text { metabolism }\end{array}$ & & & -1.05 & $0.0 \mathrm{E}+00$ & $0.0 \mathrm{E}+00$ \\
\hline Imo1766 & 310 & purN & $\begin{array}{l}\text { Phosphoribosylglycinamide } \\
\text { formyltransferase }\end{array}$ & $\begin{array}{l}\text { Nucleotide transport and } \\
\text { metabolism }\end{array}$ & & & -1.18 & 7.0E-04 & $8.1 \mathrm{E}-03$ \\
\hline Imo1770 & 310 & purL & $\begin{array}{l}\text { Phosphoribosylformyl- } \\
\text { glycinamidine synthase, gluta- } \\
\text { mine amidotransferase subunit }\end{array}$ & $\begin{array}{l}\text { Nucleotide transport and } \\
\text { metabolism }\end{array}$ & -3.51 & $3.1 \mathrm{E}-03$ & & & \\
\hline Imo1771 & 310 & purs & $\begin{array}{l}\text { Phosphoribosylformyl- } \\
\text { glycinamidine synthase, PurS } \\
\text { subunit }\end{array}$ & $\begin{array}{l}\text { Nucleotide transport and } \\
\text { metabolism }\end{array}$ & -3.86 & $9.0 \mathrm{E}-04$ & & & \\
\hline
\end{tabular}




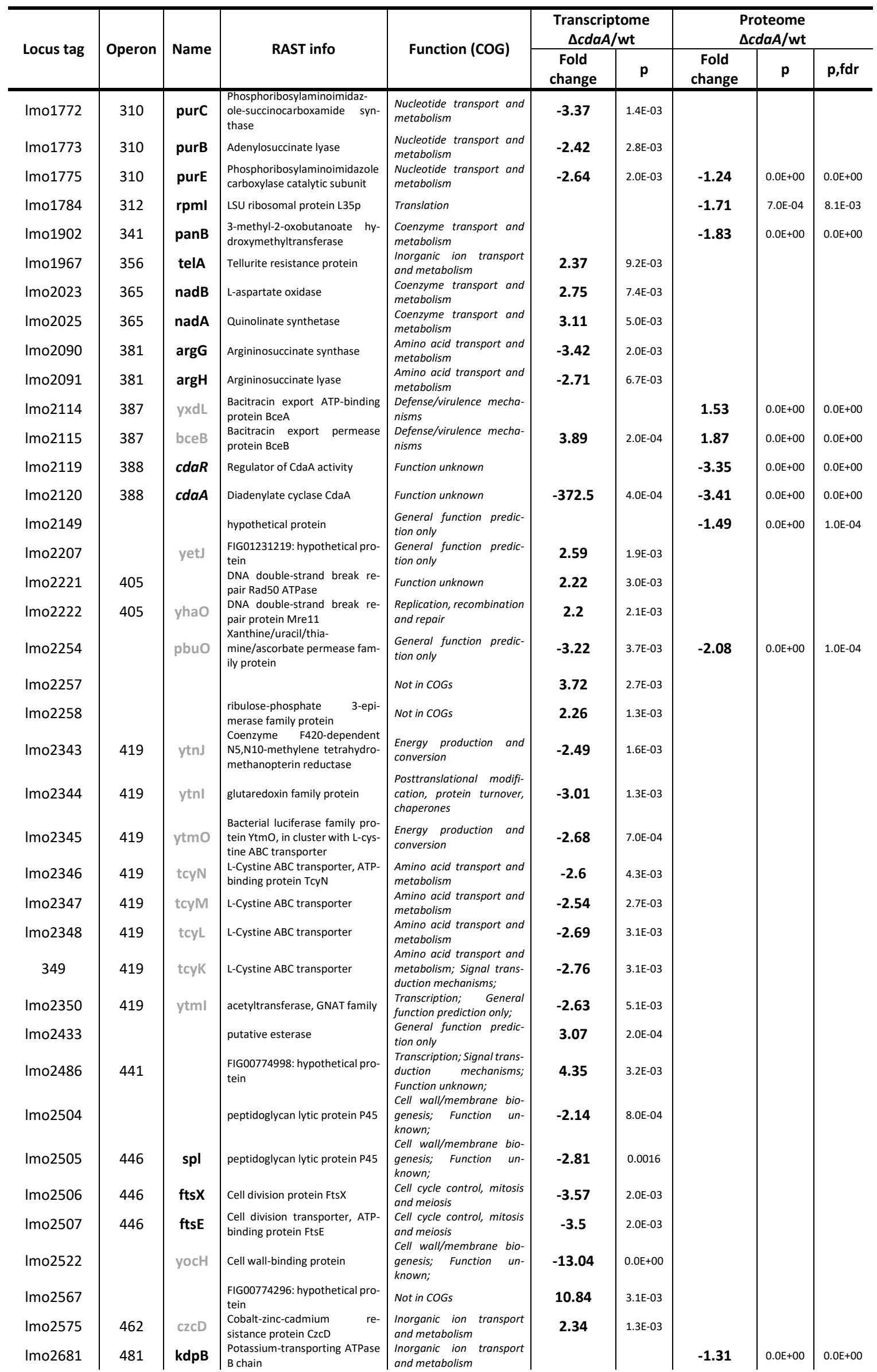




\begin{tabular}{|c|c|c|c|c|c|c|c|c|c|}
\hline \multirow{2}{*}{ Locus tag } & \multirow{2}{*}{ Operon } & \multirow{2}{*}{ Name } & \multirow{2}{*}{ RAST info } & \multirow{2}{*}{ Function (COG) } & \multicolumn{2}{|c|}{$\begin{array}{c}\text { Transcriptome } \\
\Delta c d a A / w t\end{array}$} & \multicolumn{3}{|c|}{$\begin{array}{l}\text { Proteome } \\
\Delta c d a A / w t\end{array}$} \\
\hline & & & & & $\begin{array}{c}\text { Fold } \\
\text { change }\end{array}$ & p & $\begin{array}{c}\text { Fold } \\
\text { change }\end{array}$ & p & $p, f d r$ \\
\hline Imo2683 & 482 & licB & $\begin{array}{l}\text { PTS system, cellobiose-specific } \\
\text { IIB component }\end{array}$ & $\begin{array}{l}\text { Carbohydrate transport } \\
\text { and metabolism }\end{array}$ & & & -1.93 & $0.0 \mathrm{E}+00$ & $4.0 \mathrm{E}-04$ \\
\hline Imo2684 & 482 & licC & $\begin{array}{l}\text { PTS system, cellobiose-specific } \\
\text { IIC component }\end{array}$ & $\begin{array}{l}\text { Carbohydrate transport } \\
\text { and metabolism }\end{array}$ & & & -1.14 & $0.0 \mathrm{E}+00$ & $5.0 \mathrm{E}-04$ \\
\hline Imo2720 & & ytcl & $\begin{array}{l}\text { Acyl-coenzyme A synthe- } \\
\text { tases/AMP-(fatty) acid ligases }\end{array}$ & $\begin{array}{l}\text { Lipid transport and metab- } \\
\text { olism }\end{array}$ & & & 1.47 & $0.0 \mathrm{E}+00$ & $0.0 \mathrm{E}+00$ \\
\hline Imo2785 & & kat & & $\begin{array}{l}\text { Inorganic ion transport } \\
\text { and metabolism }\end{array}$ & 2.07 & $1.0 \mathrm{E}-03$ & & & \\
\hline
\end{tabular}

RAST= Rapid Annotation using Subsystem Technology (database), COG=Clusters of Orthologous Groups (database), grey names=homologs of $B$. subtilis 169 (ListiWiki)

Tab. S4.2 Data-dependent acquisition mass spectrometry (DDA-MS) settings

\begin{tabular}{|c|c|}
\hline \multicolumn{2}{|c|}{ Reversed phase liquid chromatography (RPLC) } \\
\hline instrument & Ultimate 3000 RSLC (Thermo Scientific) \\
\hline trap column & $\begin{array}{l}75 \mu \mathrm{m} \text { inner diameter, packed with } 3 \mu \mathrm{m} \text { C18 particles }\left(\text { Acclaim }^{\mathrm{TM}}\right. \\
\text { PepMap }{ }^{\text {TM}} 100 \text {, Thermo Scientific) }\end{array}$ \\
\hline analytical column & $\begin{array}{l}\text { Accucore }^{\mathrm{TM}} \text { 150-C18, (Thermo Fisher Scientific }{ }^{\mathrm{TM}} \text { ) } \\
25 \mathrm{~cm} \times 75 \mu \mathrm{m}, 2,6 \mu \mathrm{m} \text { C18 particles, } 150 \AA \AA \text { pore size }\end{array}$ \\
\hline buffer system & $\begin{array}{l}0.1 \%(\mathrm{v} / \mathrm{v}) \text { acetic acid, } 2 \%(\mathrm{v} / \mathrm{v}) \text { ACN (buffer } \mathrm{A}) \\
100 \%(\mathrm{v} / \mathrm{v}) \text { ACN in } 0.1 \%(\mathrm{v} / \mathrm{v}) \text { acetic acid (buffer B) }\end{array}$ \\
\hline flow rate & $300 \mathrm{nl} / \mathrm{min}$ \\
\hline gradient & linear gradient of buffer B from $2 \%(v / v)$ up to $25 \%(v / v)$ \\
\hline gradient duration & $60 \mathrm{~min}$ \\
\hline column oven temperature & $40^{\circ} \mathrm{C}$ \\
\hline \multicolumn{2}{|l|}{ Mass spectrometry (MS) } \\
\hline instrument & Q Exactive ${ }^{\mathrm{TM}} \mathrm{HF}-\mathrm{X}$ mass spectrometer (Thermo Scientific ${ }^{\mathrm{TM}}$ ) \\
\hline Ion source & Triversa NanoMate ${ }^{\circledR}$ (Advion) \\
\hline operation mode & data-dependent \\
\hline \multicolumn{2}{|l|}{ Full MS } \\
\hline MS scan resolution & 60,000 \\
\hline AGC target & $3 e 6$ \\
\hline $\begin{array}{l}\text { maximum ion injection time for the } \\
\text { MS scan }\end{array}$ & $45 \mathrm{~ms}$ \\
\hline Scan range & 300 to $1650 \mathrm{~m} / \mathrm{z}$ \\
\hline Spectra data type & profile \\
\hline \multicolumn{2}{|l|}{ dd-MS2 } \\
\hline Resolution & 15,000 \\
\hline MS/MS AGC target & $1 \mathrm{e} 5$ \\
\hline $\begin{array}{l}\text { maximum ion injection time for the } \\
\text { MS/MS scans }\end{array}$ & $22 \mathrm{~ms}$ \\
\hline Spectra data type & profile \\
\hline selection for MS/MS & $\begin{array}{l}12 \text { most abundant isotope patterns with charge } \geq 2+\text { and }<7+\text { from } \\
\text { the survey scan }\end{array}$ \\
\hline isolation window & $1.3 \mathrm{~m} / \mathrm{z}$ \\
\hline Fixed first mass & $100 \mathrm{~m} / \mathrm{z}$ \\
\hline dissociation mode & higher energy collisional dissociation (HCD) \\
\hline
\end{tabular}




\begin{tabular}{ll}
\hline normalized collision energy & $27 \%$ \\
dynamic exclusion & $30 \mathrm{~s}$ \\
Charge exclusion & Unassigned, $1,>6$ \\
\hline
\end{tabular}

Tab. S4.3 Data-independent acquisition mass spectrometry (DIA-MS) settings

\begin{tabular}{|c|c|c|}
\hline \multicolumn{3}{|c|}{ Reversed phase liquid chromatography (RPLC) } \\
\hline instrument & \multicolumn{2}{|c|}{ Ultimate 3000 RSLC (Thermo Scientific) } \\
\hline trap column & \multicolumn{2}{|c|}{$\begin{array}{l}75 \mu \mathrm{m} \text { inner diameter, packed with } 3 \mu \mathrm{m} \text { C18 particles (Acclaim }{ }^{\mathrm{TM}} \text { Pep- } \\
\text { Map }^{\mathrm{TM}} 100 \text {, Thermo Scientific }{ }^{\mathrm{TM}} \text { ) }\end{array}$} \\
\hline analytical column & \multicolumn{2}{|c|}{$\begin{array}{l}\text { Accucore }^{\mathrm{TM}} \text { 150-C18, (Thermo Fisher Scientific }{ }^{\mathrm{TM}} \text { ) } \\
25 \mathrm{~cm} \times 75 \mu \mathrm{m}, 2,6 \mu \mathrm{m} \text { C18 particles, } 150 \text { A pore size }\end{array}$} \\
\hline buffer system & \multicolumn{2}{|c|}{$0.1 \%(\mathrm{v} / \mathrm{v})$ acetic acid, $2 \%(\mathrm{v} / \mathrm{v})$ ACN (buffer A) } \\
\hline flow rate & \multicolumn{2}{|c|}{$300 \mathrm{nl} / \mathrm{min}$} \\
\hline gradient & \multicolumn{2}{|c|}{ linear gradient of buffer B from $2 \%(\mathrm{v} / \mathrm{v})$ up to $25 \%(\mathrm{v} / \mathrm{v})$} \\
\hline gradient duration & \multicolumn{2}{|l|}{$60 \mathrm{~min}$} \\
\hline column oven temperature & \multicolumn{2}{|l|}{$40^{\circ} \mathrm{C}$} \\
\hline \multicolumn{3}{|l|}{ Mass spectrometry (MS) } \\
\hline instrument & \multicolumn{2}{|c|}{ Q Exactive ${ }^{\mathrm{TM}}$ HF-X mass spectrometer (Thermo Scientific ${ }^{\mathrm{TM}}$ ) } \\
\hline electrospray & \multicolumn{2}{|c|}{ Nanospray Flex Ion Source } \\
\hline operation mode & \multicolumn{2}{|c|}{ data-independent } \\
\hline \multicolumn{3}{|l|}{ Full MS } \\
\hline MS scan resolution & \multicolumn{2}{|l|}{120,000} \\
\hline AGC target & \multicolumn{2}{|l|}{$3 \mathrm{e} 6$} \\
\hline $\begin{array}{l}\text { maximum ion injection time for } \\
\text { the MS scan }\end{array}$ & \multicolumn{2}{|l|}{$60 \mathrm{~ms}$} \\
\hline Scan range & \multicolumn{2}{|c|}{350 to $1200 \mathrm{~m} / \mathrm{z}$} \\
\hline Spectra data type & \multicolumn{2}{|c|}{ profile } \\
\hline \multicolumn{3}{|l|}{ DIA settings } \\
\hline Resolution & \multicolumn{2}{|l|}{30,000} \\
\hline MS/MS AGC target & \multicolumn{2}{|l|}{$3 \mathrm{e} 6$} \\
\hline $\begin{array}{l}\text { maximum ion injection time for } \\
\text { the MS/MS scans }\end{array}$ & \multicolumn{2}{|l|}{ auto } \\
\hline Spectra data type & \multicolumn{2}{|l|}{ profile } \\
\hline selection for MS/MS & \multicolumn{2}{|l|}{1} \\
\hline \multirow{9}{*}{ isolation windows } & $\begin{array}{l}\text { window } \\
\text { number }\end{array}$ & $\begin{array}{l}\text { Center of } \quad \text { window width }[\mathrm{m} / \mathrm{z}] \\
\text { window }[\mathrm{m} / \mathrm{z}]\end{array}$ \\
\hline & 1 & 359.5 \\
\hline & 2 & 377.6 \\
\hline & 3 & 395.7 \\
\hline & 4 & 413.8 \\
\hline & 5 & 431.9 \\
\hline & 6 & 450.0 \\
\hline & 7 & 468.1 \\
\hline & 8 & 486.1 \\
\hline
\end{tabular}


Cyclic di-AMP and osmoregulation in Listeria monocytogenes

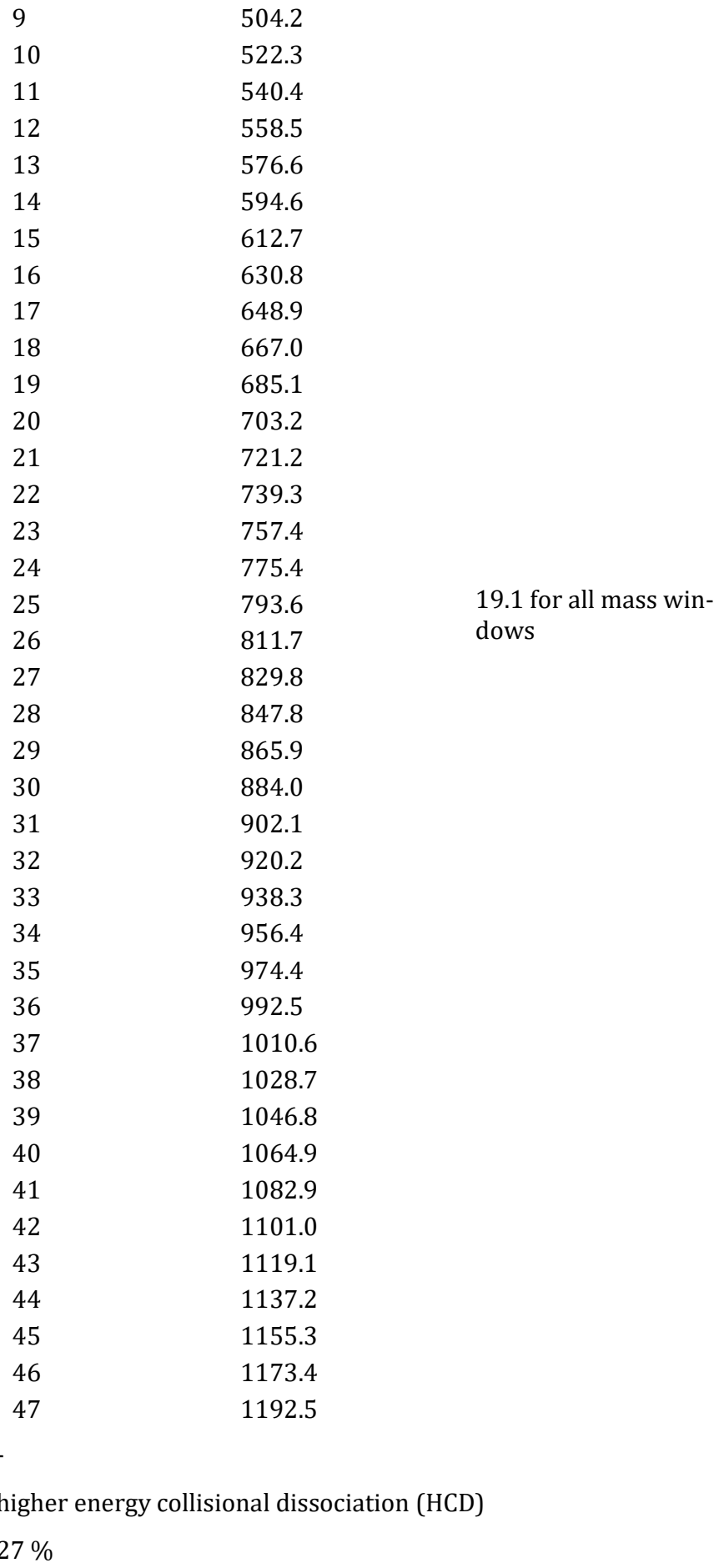

Fixed first mass

dissociation mode higher energy collisional dissociation (HCD)

normalized collision energy $\quad 27 \%$

Tab. S4.4 Spectronaut Pulsar setting for library generation

Ion library generation settings in Spectronaut using Pulsar search engine

Fasta database L. monocytogenes EGD-e

Fasta file number of protein entries 2873

Cleavage rule Trypsin/P

Missed cleavages 2 


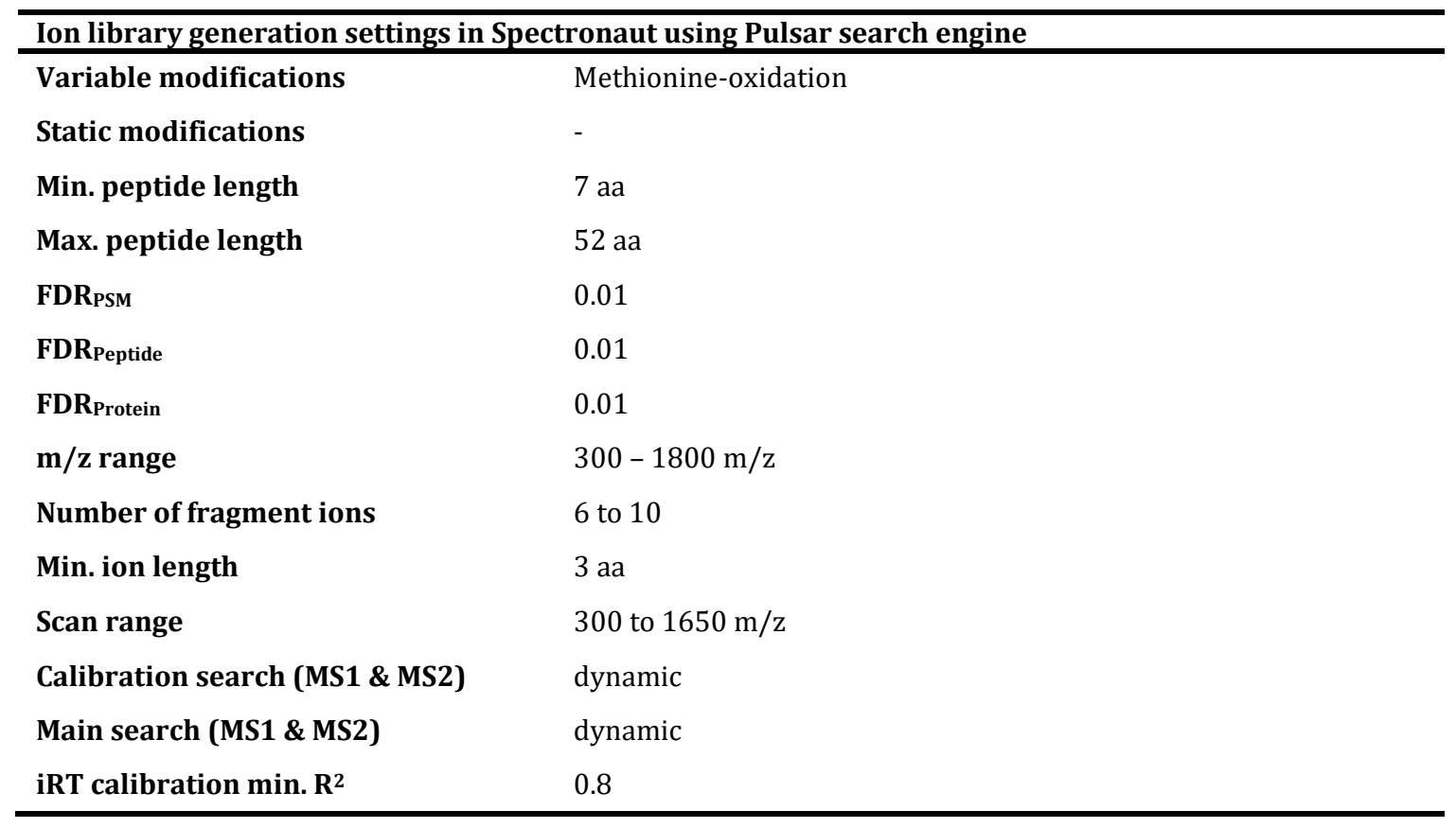

Tab. S4.5 Spectronaut Pulsar DIA-MS analysis settings

\begin{tabular}{|c|c|}
\hline DIA-MS analysis settings & \\
\hline Fasta database & Listeria monocytogenes EGD-e \\
\hline Fasta file number of protein entries & 2873 \\
\hline Cleavage rule & Trypsin/P \\
\hline Missed cleavages & 2 \\
\hline Variable modifications & Methionine-oxidation \\
\hline Data Extraction MS1 tol. & dynamic \\
\hline Data Extraction MS2 tol. & dynamic \\
\hline XIC extraction window & dynamic \\
\hline Calibration mode & Automatic with iRT precision \\
\hline Protein Identification Q-value cutoff & 0.01 \\
\hline $\begin{array}{l}\text { Precursor Identification Q-value cut- } \\
\text { off }\end{array}$ & 0.001 \\
\hline Use interference correction? & TRUE \\
\hline Min. precursor-ions to keep & 2 \\
\hline Min. fragment-ions to keep & 3 \\
\hline Quantity MS-level & MS2 \\
\hline Data filtering & Q-value percentile \\
\hline Data filtering fraction & 0.5 \\
\hline Workflow profiling strategy & iRT profiling \\
\hline Workflow profiling row selection & Min. Q-value Row selection (cutoff $=0.001)$ \\
\hline Workflow profiling target selection & $\begin{array}{l}\text { Profile only non-identified Precursor (identification criterion: Q- } \\
\text { value }<0.0001 \text { ) }\end{array}$ \\
\hline $\begin{array}{l}\text { Workflow profiling carry-over exact } \\
\text { Peak Boundaries }\end{array}$ & TRUE \\
\hline Protein Inference Workflow & automatic \\
\hline
\end{tabular}




\section{Chapter 5}
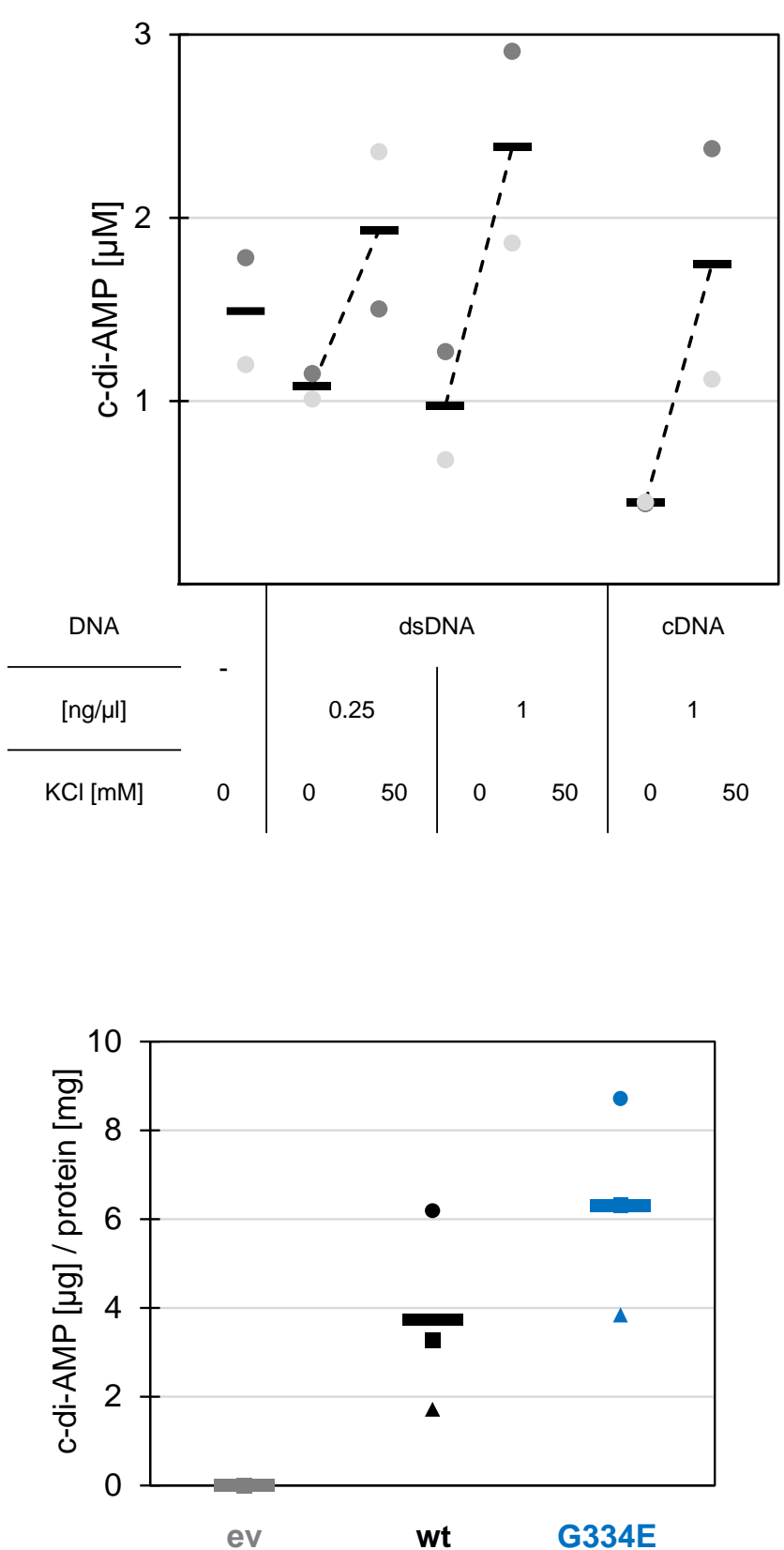

Fig. S5.1 cDNA has a greater inhibitory effect on DisA activity compared to dsDNA. The DACs DisA of $B$. subtilis was purified via His-tag purification and subsequently dialyzed overnight in $10 \mathrm{mM}$ Tris- $\mathrm{HCl} \mathrm{pH} \mathrm{7.5,5 \%} \mathrm{(w/v)} \mathrm{glyc-}$ erol and $1 \mathrm{mM} \mathrm{DTT}$ at $4^{\circ} \mathrm{C} .100 \mathrm{nM}$ of DisA was incubated in $10 \mathrm{mM}$ Tris- $\mathrm{HCl} \mathrm{pH} 7.5$ with $100 \mu \mathrm{M} \mathrm{ATP}, 10 \mathrm{mM} \mathrm{MgCl}$ and $0.1 \%(w / v)$ $\mathrm{BSA}$ at $37^{\circ} \mathrm{C}$ for $30 \mathrm{~min}$ when the reactions were stopped and c-di-AMP extracted. Samples were incubated with or without the indicated concentration of $B$. subtilis CDNA or a 2 $\mathrm{kb}$ PCR product and with or without the indicated concentration of $\mathrm{KCl}$. Data of two technical replicates are shown. Horizontal lines depict the mean of the two technical replicates. cDNA = chromosomal DNA, dsDNA = doublestranded DNA.

Fig. S5.2 DisA G334E is more active in vivo. E. coli XL1-blue transformed with the empty vector pBAD33 (ev), pBP394 (DisA wt) or pBP395 DisA (G334E) was grown in LB medium at $37^{\circ} \mathrm{C}$ until cells grew exponentially. $0.005 \%$ $(w / v)$ L-arabinose was added to induce DAC expression and cultures incubated for another one and a half hours. Samples were taken and c-di-AMP concentrations determined. Data of three biological replicates are shown as the mean of two technical replicates per measurement point. Horizontal lines depict the mean of the three biological replicates. 

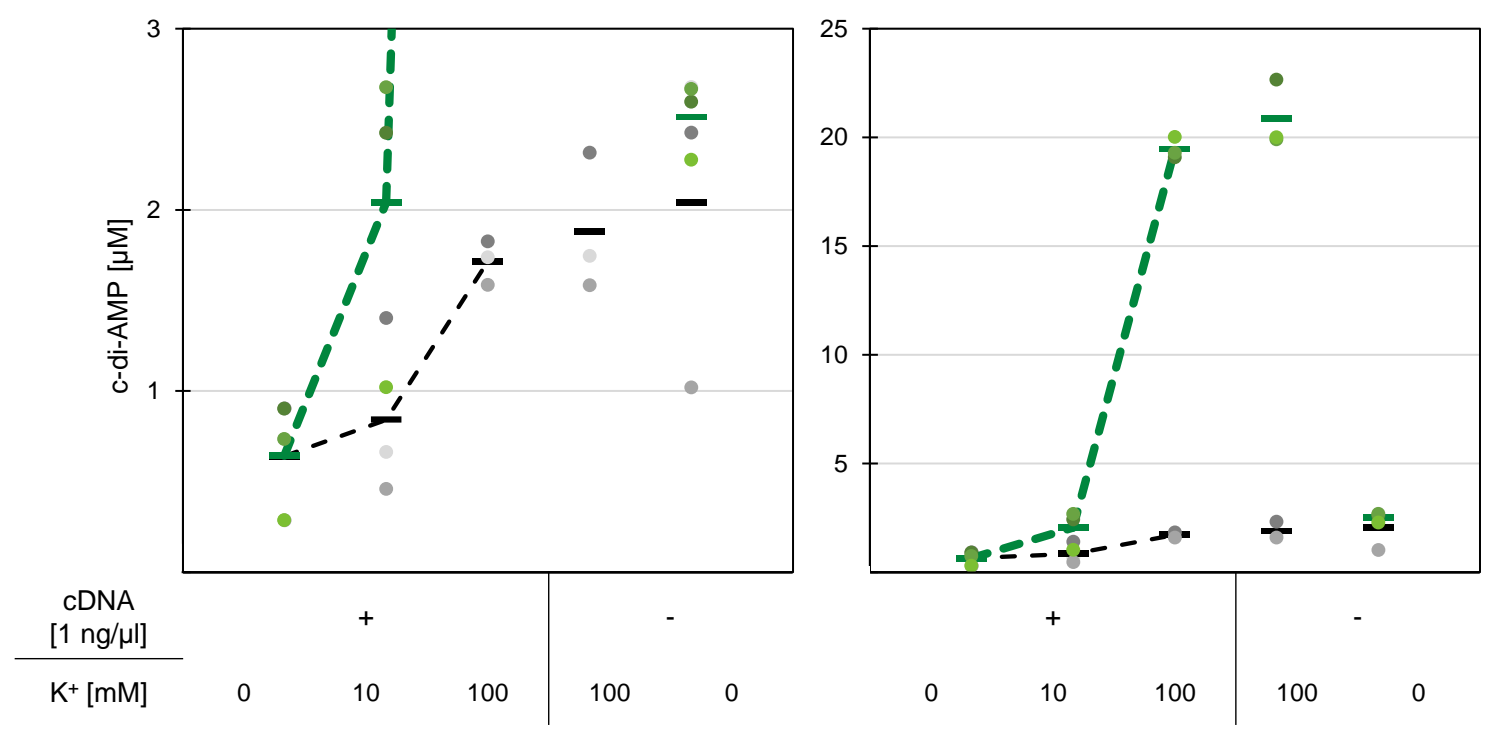

Fig. S5.3 DisA activity is influenced by other potassium ion-containing solutes. The DisA enzyme was purified via Histag purification and subsequently dialyzed overnight in $10 \mathrm{mM}$ Tris- $\mathrm{HCl} \mathrm{pH} \mathrm{7.5,5 \% (w/v)} \mathrm{glycerol} \mathrm{and} 1 \mathrm{mM} \mathrm{DTT}$ at $4^{\circ} \mathrm{C}$. $100 \mathrm{nM}$ of DisA was incubated in $10 \mathrm{mM}$ Tris- $\mathrm{HCl} \mathrm{pH} 7.5$ with $100 \mu \mathrm{M} \mathrm{ATP}, 10 \mathrm{mM} \mathrm{MgCl}_{2}$ and $0.1 \%(w / v) \mathrm{BSA}^{\circ} 37^{\circ} \mathrm{C}$ for $30 \mathrm{~min}$ when the reactions were stopped and c-di-AMP extracted. Samples were incubated with or without $1 \mathrm{ng} / \mu \mathrm{l}$ of $B$. subtilis CDNA and with or without the indicated concentration of $\mathrm{KCl}$ (black) or a mixture of potassium dihydrogen phosphate and dipotassium hydrogen phosphate. The potassium hydrogen phosphate salts where herby titrated to achieve a solution of $\mathrm{pH} 7.5$, the potassium concentration calculated and the depicted amount equimolar to the potassium chloride concentration added. Both figures depict the same data with a different range of the depicted c-di-AMP concentration for better discrimination. Data of three biological replicates are shown as the mean of two technical replicates per measurement point. Horizontal lines depict the mean of the three biological replicates. cDNA $=$ chromosomal DNA.
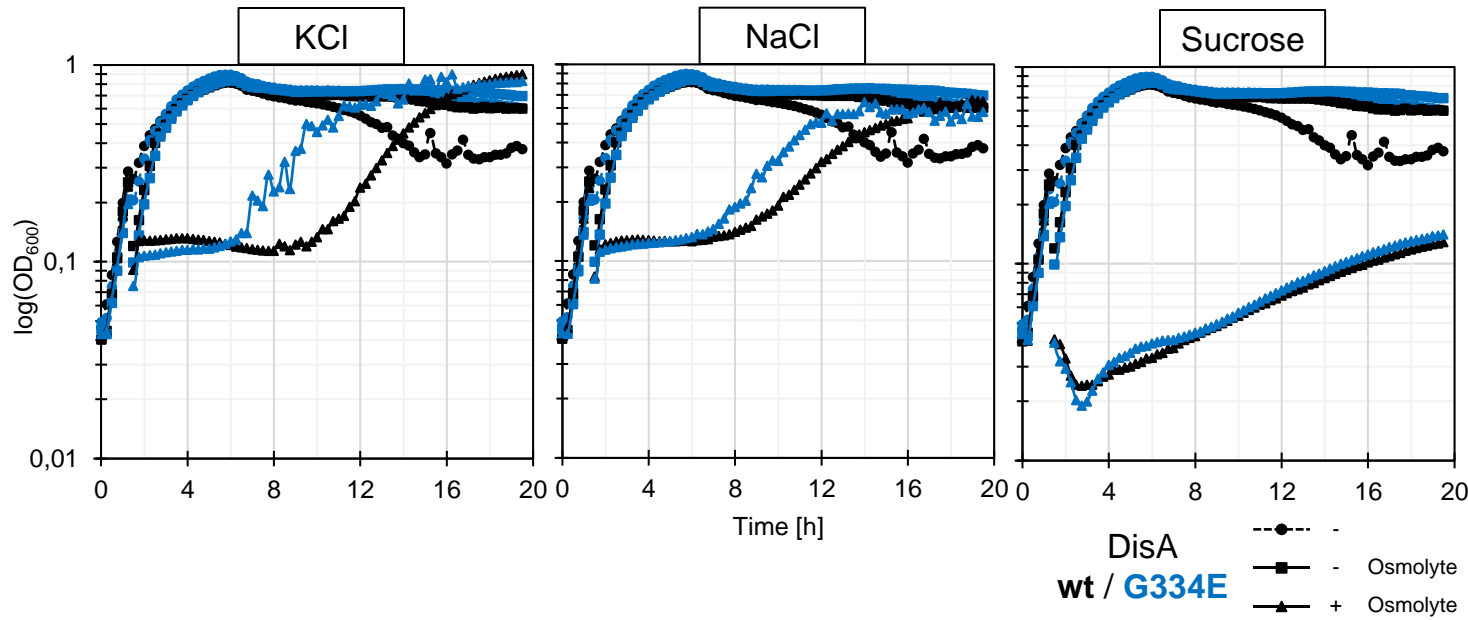

Fig. S5.4 The DisA G334E mutation affects resistance of B. subtilis to salt stress. B. subtilis containing either DisA (wt) or DisA (G334E) as sole DAC was grown in $200 \mu \mathrm{LB}$ medium at $37^{\circ} \mathrm{C}$ with medium shaking using a Synergy Mx multiwell plate reader. Cultures were grown for 1.25 hours and $100 \mu$ l of cells were added to $100 \mu$ l of pre-warmed LB containing the indicated osmolytes to a final concentration of $1.5 \mathrm{M}$, LB without osmolytes or $200 \mu$ l of cells were transferred without any additions and growth was resumed. Depicted are mean values of three biological replicates with two technical replicates, each. The $\mathrm{OD}_{600}$ was measured in 15 min intervals. 


\section{Chapter 6}
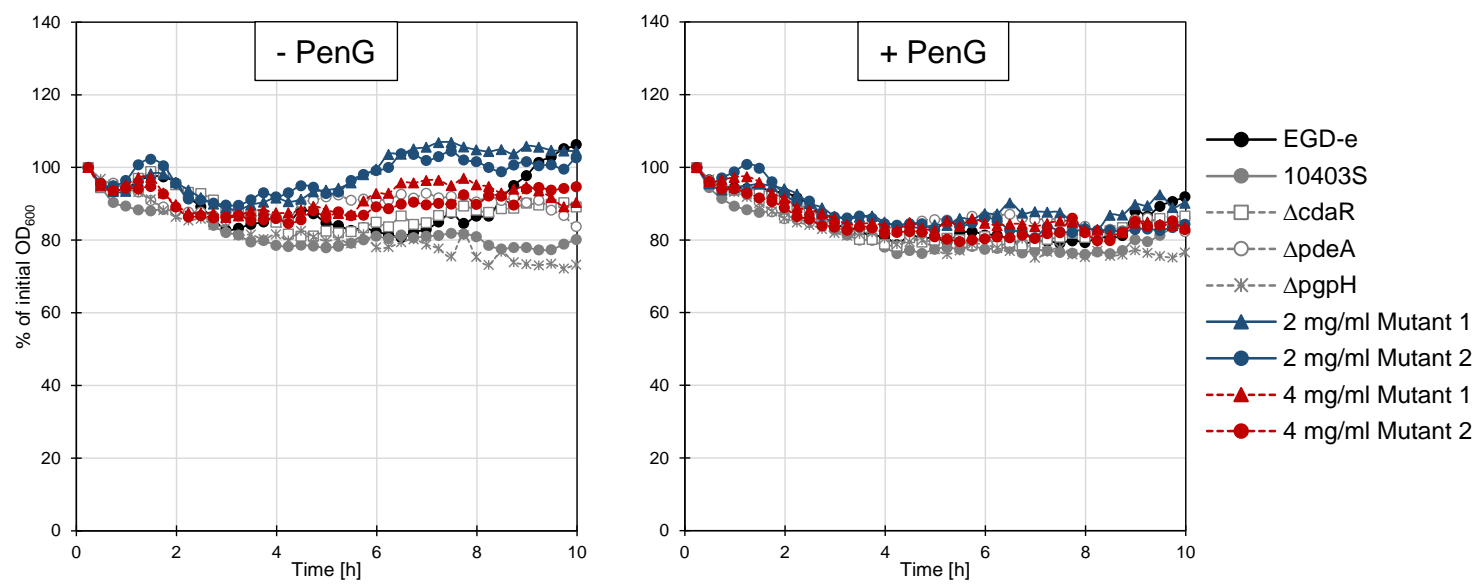

Fig. S6.1 The $P_{\text {rli31 }}$ suppressor mutants are do not show an altered autolysis with or without penicillin $\mathrm{G}$. The depicted strains were grown in $\mathrm{BHI}$ at $37^{\circ} \mathrm{C}$ until they reached an $\mathrm{OD}_{600}$ between of about 0.8 . Cells were washed in $50 \mathrm{mM}$ Tris $\mathrm{pH} 8$ and the suspensions adjusted to an $\mathrm{OD}_{600}$ of 1.2. Nothing (-PenG) or $25 \mu \mathrm{g} / \mathrm{ml}$ penicillin $\mathrm{G}$ were added and the cell suspensions incubated at $37^{\circ} \mathrm{C}$ and the decline in $\mathrm{OD}_{600}$ measured in $15 \mathrm{~min}$ intervals. Depicted are the mean values of four biological replicates.

Tab. S6.1 Complete list of differentially expressed genes or synthesized proteins in $\Delta$ rli31 vs. Prli31*

\begin{tabular}{|c|c|c|c|c|c|c|c|}
\hline \multirow{2}{*}{ Locus tag } & \multirow{2}{*}{ Operon } & \multirow{2}{*}{ Name } & \multirow{2}{*}{ RAST info } & \multirow{2}{*}{ Function (COG) } & \multicolumn{2}{|c|}{$\begin{array}{c}\text { Transcriptome } \\
\Delta \text { rli31/P } \mathbf{P}_{\text {rli31 }} *\end{array}$} & \multirow{2}{*}{$\begin{array}{c}\text { Proteome } \\
\Delta r l i 31 / \mathrm{P}_{\text {rli31 }} \\
\text { *old change } \\
\mathrm{NS}^{1}\end{array}$} \\
\hline & & & & & $\begin{array}{c}\text { Fold } \\
\text { change }\end{array}$ & $\mathbf{p}$ & \\
\hline $\operatorname{Imo0130}$ & & yhgE & 5'-nucleotidase & $\begin{array}{l}\text { Nucleotide transport and me- } \\
\text { tabolism }\end{array}$ & & & 1.7 \\
\hline Imo0131 & & pdeB & $\begin{array}{l}\text { FIG00774523: hypothetical pro- } \\
\text { tein, c-di-GMP PDE }\end{array}$ & Signal transduction mechanisms & & & 1.87 \\
\hline Imo0257 & & & RNA-2',3'-PO4:RNA-5'-OH ligase & Function unknown & & & -1.49 \\
\hline Imo0286 & & ykrV & $\begin{array}{l}\text { Glutamine-dependent 2-keto-4- } \\
\text { methylthiobutyrate transami- } \\
\text { nase }\end{array}$ & $\begin{array}{l}\text { Amino acid transport and me- } \\
\text { tabolism }\end{array}$ & 1.58 & $7.2 \mathrm{E}-02$ & \\
\hline Imo0324 & & & $\begin{array}{l}\text { FIG00774236: hypothetical pro- } \\
\text { tein }\end{array}$ & Not in COGs & 2.75 & $4.4 \mathrm{E}-02$ & \\
\hline Imo0342 & 58 & tkt & Transketolase & $\begin{array}{l}\text { Carbohydrate transport and } \\
\text { metabolism }\end{array}$ & 1.7 & $5.6 \mathrm{E}-02$ & \\
\hline Imo0349 & 58 & & $\begin{array}{l}\text { FIG00774379: hypothetical pro- } \\
\text { tein }\end{array}$ & Not in COGs & 3.6 & $2.3 \mathrm{E}-02$ & \\
\hline Imo0370 & 63 & & $\begin{array}{l}\text { Alkylphosphonate utilization } \\
\text { operon protein PhnA }\end{array}$ & $\begin{array}{l}\text { Inorganic ion transport and me- } \\
\text { tabolism }\end{array}$ & & & -1.9 \\
\hline Imo0415 & & $\operatorname{pgdA}$ & $\begin{array}{l}\text { Peptidoglycan } \mathrm{N} \text {-acetylglucosa- } \\
\text { mine deacetylase }\end{array}$ & Cell wall/membrane biogenesis & -1.96 & $8.3 \mathrm{E}-02$ & -4.14 \\
\hline Imo0422 & 74 & IstR & $\begin{array}{l}\text { Transcriptional regulator, PadR } \\
\text { family }\end{array}$ & Transcription & & & -1.82 \\
\hline Imo0427 & 75 & fruA & $\begin{array}{l}\text { PTS system, fructose-specific IIB } \\
\text { component }\end{array}$ & $\begin{array}{l}\text { Carbohydrate transport and } \\
\text { metabolism }\end{array}$ & & & 1.66 \\
\hline Imo0440 & & & $\begin{array}{l}\text { FIG00774042: hypothetical pro- } \\
\text { tein }\end{array}$ & Not in COGs & & & -1.63 \\
\hline Imo0444 & & yhgE & $\begin{array}{l}\text { FIG00776024: hypothetical pro- } \\
\text { tein }\end{array}$ & Function unknown & & & 1.49 \\
\hline Imo0485 & & yfhC & $\begin{array}{l}\text { Putative nitroreductase family } \\
\text { protein SACOL0874 }\end{array}$ & $\begin{array}{l}\text { Energy production and conver- } \\
\text { sion }\end{array}$ & & & 1.88 \\
\hline Imo0540 & & pbpX & $\begin{array}{l}\text { penicillin-binding protein, puta- } \\
\text { tive }\end{array}$ & Defense/virulence mechanisms & -1.84 & $9.1 \mathrm{E}-03$ & $N D$ in $\Delta^{2}$ \\
\hline Imo05593 & & & $\begin{array}{l}\text { Magnesium and cobalt } \\
\text { transport protein CorA }\end{array}$ & $\begin{array}{l}\text { Inorganic ion transport and me- } \\
\text { tabolism }\end{array}$ & -70.92 & $3.0 \mathrm{E}-04$ & $N D$ in $\Delta^{2}$ \\
\hline Imo0585 & & & extracellular protein & Not in COGs & 1.76 & $4.9 \mathrm{E}-02$ & \\
\hline Imo0590 & 95 & & $\begin{array}{l}\text { Dihydroxyacetone kinase family } \\
\text { protein }\end{array}$ & $\begin{array}{l}\text { General function prediction } \\
\text { only; Function unknown; }\end{array}$ & & & 1.94 \\
\hline
\end{tabular}




\begin{tabular}{|c|c|c|c|c|c|c|c|}
\hline \multirow{2}{*}{ Locus tag } & \multirow{2}{*}{ Operon } & \multirow{2}{*}{ Name } & \multirow{2}{*}{ RAST info } & \multirow{2}{*}{ Function (COG) } & \multicolumn{2}{|c|}{$\begin{array}{l}\text { Transcriptome } \\
\Delta r l i 31 / P_{\text {rli31 }}{ }^{*}\end{array}$} & \multirow{2}{*}{$\begin{array}{c}\text { Proteome } \\
\Delta \text { rli31/Prli31 } \\
\text { Fold change } \\
\text { NS }^{1}\end{array}$} \\
\hline & & & & & $\begin{array}{c}\text { Fold } \\
\text { change }\end{array}$ & p & \\
\hline Imo0591 & 95 & & $\begin{array}{l}\text { FIG00775146: hypothetical pro- } \\
\text { tein }\end{array}$ & Function unknown & & & 1.49 \\
\hline Imo0620 & & & $\begin{array}{l}\text { FIG00774170: hypothetical pro- } \\
\text { tein }\end{array}$ & $\begin{array}{l}\text { General function prediction } \\
\text { only } \\
\text { Lipid transport and metabolism; }\end{array}$ & -1.6 & $4.0 E-02$ & -1.91 \\
\hline Imo0669 & 109 & yhxD & $\begin{array}{l}\text { oxidoreductase, short-chain de- } \\
\text { hydrogenase/reductase family }\end{array}$ & $\begin{array}{l}\text { Secondary metabolites biosyn- } \\
\text { thesis, transport and catabo- } \\
\text { lism; General function predic- } \\
\text { tion only; }\end{array}$ & & & 2.13 \\
\hline Imo0682 & 111 & flgG & $\begin{array}{l}\text { Flagellar basal-body rod protein } \\
\text { FlgG }\end{array}$ & Cell motility & 3.5 & $5.5 E-02$ & \\
\hline Imo0685 & 111 & $\operatorname{mot} A$ & $\begin{array}{l}\text { Flagellar motor rotation protein } \\
\text { MotA }\end{array}$ & Cell motility & 4.44 & $3.0 \mathrm{E}-02$ & \\
\hline Imo0688 & 111 & gmaR & $\begin{array}{l}\text { glycosyl transferase, group } 2 \\
\text { family protein }\end{array}$ & $\begin{array}{l}\text { Cell wall/membrane biogenesis; } \\
\text { General function prediction } \\
\text { only; }\end{array}$ & 1.52 & $4.9 \mathrm{E}-02$ & 6.05 \\
\hline Imo0689 & 111 & cheV & Chemotaxis protein CheV & $\begin{array}{l}\text { Cell motility; Signal transduction } \\
\text { mechanisms; }\end{array}$ & 1.51 & $3.9 \mathrm{E}-02$ & 1.85 \\
\hline Imo0698 & 112 & & $\begin{array}{l}\text { Flagellar motor switch protein } \\
\text { FliN }\end{array}$ & $\begin{array}{l}\text { Cell motility; Intracellular traf- } \\
\text { ficking and secretion; }\end{array}$ & 4.05 & $7.9 \mathrm{E}-02$ & \\
\hline Imo0699 & 112 & flim & $\begin{array}{l}\text { Flagellar motor switch protein } \\
\text { FliM }\end{array}$ & Cell motility & 2.77 & $1.5 \mathrm{E}-02$ & \\
\hline Imo0706 & 112 & flgL & $\begin{array}{l}\text { Flagellar hook-associated pro- } \\
\text { tein FlgL }\end{array}$ & Cell motility & 1.64 & $6.4 \mathrm{E}-02$ & \\
\hline$\underline{\operatorname{mo} 0710}$ & 112 & flgB & $\begin{array}{l}\text { Flagellar basal-body rod protein } \\
\text { FlgB }\end{array}$ & Cell motility & 1.67 & $1.9 \mathrm{E}-02$ & \\
\hline Imo0711 & 112 & flgC & $\begin{array}{l}\text { Flagellar basal-body rod protein } \\
\text { FlgC }\end{array}$ & Cell motility & 2.33 & $6.7 \mathrm{E}-02$ & \\
\hline Imo0715 & 112 & fliH & Flagellar assembly protein FliH & Not in COGs & 1.6 & $3.9 \mathrm{E}-02$ & \\
\hline Imo0716 & 112 & flil & $\begin{array}{l}\text { Flagellum-specific ATP synthase } \\
\text { Flil }\end{array}$ & $\begin{array}{l}\text { Cell motility; Intracellular traf- } \\
\text { ficking and secretion; }\end{array}$ & 1.81 & $1.8 \mathrm{E}-02$ & \\
\hline Imo0717 & 112 & vjbJ & $\begin{array}{l}\text { Soluble lytic murein transglyco- } \\
\text { sylase precursor }\end{array}$ & Cell wall/membrane biogenesis & 1.71 & $7.3 \mathrm{E}-02$ & \\
\hline Imo0718 & 112 & & $\begin{array}{l}\text { FIG00774152: hypothetical pro- } \\
\text { tein }\end{array}$ & Not in COGs & 1.79 & $1.9 \mathrm{E}-02$ & \\
\hline Imo0755 & 119 & ypmR & $\begin{array}{l}\text { FIG006988: Lipase/Acylhydro- } \\
\text { lase with GDSL-like motif }\end{array}$ & $\begin{array}{l}\text { Amino acid transport and me- } \\
\text { tabolism }\end{array}$ & 2.44 & $1.4 \mathrm{E}-02$ & \\
\hline Imo0772 & 122 & & $\begin{array}{l}\text { Transcriptional regulator, GntR } \\
\text { family }\end{array}$ & Transcription & & & -2.21 \\
\hline Imo0775 & & & $\begin{array}{l}\text { FIG00774607: hypothetical pro- } \\
\text { tein }\end{array}$ & Not in COGs & & & 1.58 \\
\hline Imo0784 & 124 & levD & $\begin{array}{l}\text { IIB component / PTS system, } \\
\text { mannose-specific IIA compo- } \\
\text { nent }\end{array}$ & $\begin{array}{l}\text { Carbohydrate transport and } \\
\text { metabolism }\end{array}$ & & & 1.62 \\
\hline Imo0811 & & & Carbonic anhydrase precursor & $\begin{array}{l}\text { Inorganic ion transport and me- } \\
\text { tabolism }\end{array}$ & & & 1.63 \\
\hline Imo0834 & 129 & & $\begin{array}{l}\text { FIG00774832: hypothetical pro- } \\
\text { tein }\end{array}$ & Not in COGs & 1.9 & $5.4 \mathrm{E}-02$ & \\
\hline Imo0880 & & & $\begin{array}{l}\text { Putative peptidoglycan bound } \\
\text { protein (LPXTG motif) }\end{array}$ & Cell wall/membrane biogenesis & & & 2.32 \\
\hline Imo0891 & 136 & rsbT & Anti-sigma B factor RsbT & Signal transduction mechanisms & & & 1.55 \\
\hline Imo0895 & 136 & sigB & $\begin{array}{l}\text { RNA polymerase sigma factor } \\
\text { SigB }\end{array}$ & Transcription & & & 1.57 \\
\hline Imo0901 & & gmuC & $\begin{array}{l}\text { PTS system, cellobiose-specific } \\
\text { IIC component }\end{array}$ & $\begin{array}{l}\text { Carbohydrate transport and } \\
\text { metabolism }\end{array}$ & 1.84 & $2.8 \mathrm{E}-02$ & \\
\hline Imo0934 & 144 & yhbA & $\begin{array}{l}\text { Epoxyqueuosine (oQ) reductase } \\
\text { QueG }\end{array}$ & $\begin{array}{l}\text { Energy production and conver- } \\
\text { sion }\end{array}$ & 1.62 & $2.7 \mathrm{E}-02$ & \\
\hline Imo0935 & 144 & $\operatorname{cspR}$ & $\begin{array}{l}\text { tRNA (cytosine34-2'-O-)-methyl- } \\
\text { transferase }\end{array}$ & Translation & 1.58 & $5.9 \mathrm{E}-02$ & \\
\hline Imo1034 & 166 & glpK & $\begin{array}{l}\text { Unknown pentose kinase } \\
\text { TM0952 }\end{array}$ & $\begin{array}{l}\text { Energy production and conver- } \\
\text { sion }\end{array}$ & 1.89 & $4.3 \mathrm{E}-02$ & \\
\hline Imo1045 & 168 & & $\begin{array}{l}\text { Molybdenum cofactor biosyn- } \\
\text { thesis protein MoaD }\end{array}$ & $\begin{array}{l}\text { Coenzyme transport and me- } \\
\text { tabolism }\end{array}$ & 1.74 & $3.4 \mathrm{E}-02$ & \\
\hline Imo1047 & 168 & moaA & $\begin{array}{l}\text { Molybdenum cofactor biosyn- } \\
\text { thesis protein MoaA }\end{array}$ & $\begin{array}{l}\text { Coenzyme transport and me- } \\
\text { tabolism }\end{array}$ & 1.54 & $4.5 \mathrm{E}-02$ & \\
\hline Imo1095 & & & $\begin{array}{l}\text { PTS system, cellobiose-specific } \\
\text { IIB component }\end{array}$ & $\begin{array}{l}\text { Carbohydrate transport and } \\
\text { metabolism }\end{array}$ & & & 1.55 \\
\hline Imo1131 & & cydC & $\begin{array}{l}\text { Transport ATP-binding protein } \\
\text { CydC }\end{array}$ & $\begin{array}{l}\text { Energy production and conver- } \\
\text { sion; Posttranslational modifica- } \\
\text { tion, protein turnover, chaper- } \\
\text { ones; }\end{array}$ & & & 3 \\
\hline Imo1132 & & ygaD & $\begin{array}{l}\text { Transport ATP-binding protein } \\
\text { CydD }\end{array}$ & Defense/virulence mechanisms & & & 2.02 \\
\hline
\end{tabular}




\begin{tabular}{|c|c|c|c|c|c|c|c|}
\hline \multirow{2}{*}{ Locus tag } & \multirow{2}{*}{ Operon } & \multirow{2}{*}{ Name } & \multirow{2}{*}{ RAST info } & \multirow{2}{*}{ Function (COG) } & \multicolumn{2}{|c|}{$\begin{array}{c}\text { Transcriptome } \\
\Delta r l i 31 / P_{\text {rli31 }} *\end{array}$} & \multirow{2}{*}{$\begin{array}{c}\text { Proteome } \\
\Delta \text { rli31// } \text { rli31 }^{*} \\
\text { Fold change } \\
\text { NS }^{1}\end{array}$} \\
\hline & & & & & $\begin{array}{l}\text { Fold } \\
\text { change }\end{array}$ & p & \\
\hline Imo1148 & & & Cobalamin synthase & $\begin{array}{l}\text { Coenzyme transport and me- } \\
\text { tabolism }\end{array}$ & -2.26 & $7.4 \mathrm{E}-02$ & \\
\hline Imo1157 & & & $\begin{array}{l}\text { Propanediol dehydratase reacti- } \\
\text { vation factor small subunit }\end{array}$ & Not in COGs & -2.56 & $6.2 \mathrm{E}-02$ & \\
\hline Imo1241 & & & putative exported protein & Function unknown & & & 1.72 \\
\hline Imo1265 & & & $\begin{array}{l}\text { FIG00774764: hypothetical pro- } \\
\text { tein }\end{array}$ & Not in COGs & 1.73 & $5.9 \mathrm{E}-02$ & \\
\hline Imo1316 & 209 & cdsA & $\begin{array}{l}\text { Phosphatidate cytidylyltransfer- } \\
\text { ase }\end{array}$ & Lipid transport and metabolism & & & 2.02 \\
\hline Imo1336 & & yqgN & $\begin{array}{l}5 \text {-formyltetrahydrofolate cyclo- } \\
\text { ligase }\end{array}$ & $\begin{array}{l}\text { Coenzyme transport and me- } \\
\text { tabolism }\end{array}$ & 2.37 & $6.1 \mathrm{E}-03$ & 2.26 \\
\hline Imo1354 & 215 & yqhT & $\begin{array}{l}\text { Aminopeptidase YpdF (MP-, } \\
\text { MA-, MS-, AP-, NP- specific) }\end{array}$ & $\begin{array}{l}\text { Amino acid transport and me- } \\
\text { tabolism }\end{array}$ & & & 1.51 \\
\hline Imo1418 & 231 & & $\begin{array}{l}\text { Alkaline phosphodiesterase I/ } \\
\text { Nucleotide pyrophosphatase }\end{array}$ & $\begin{array}{l}\text { General function prediction } \\
\text { only }\end{array}$ & & & 1.63 \\
\hline Imo1494 & 246 & $\mathrm{mtn}$ & $\begin{array}{l}\text { 5'-methylthioadenosine nucleo- } \\
\text { sidase / S-adenosylhomocyste- } \\
\text { ine nucleosidase }\end{array}$ & $\begin{array}{l}\text { Nucleotide transport and me- } \\
\text { tabolism }\end{array}$ & 1.49 & $3.6 \mathrm{E}-02$ & \\
\hline Imo1600 & & aroA & $\begin{array}{l}\text { Chorismate mutase I / 2-keto-3- } \\
\text { deoxy-D-arabino-heptu- } \\
\text { losonate-7-phosphate synthase } \\
\text { I beta }\end{array}$ & $\begin{array}{l}\text { Amino acid transport and me- } \\
\text { tabolism }\end{array}$ & & & 1.49 \\
\hline Imo1701 & 296 & & $\begin{array}{l}\text { FIG00774597: hypothetical pro- } \\
\text { tein }\end{array}$ & Not in COGs & & & -1.54 \\
\hline Imo1713 & & $\underset{\mathrm{H}}{\mathrm{mreB}}$ & $\begin{array}{l}\text { Cell-shape determining protein } \\
\text { MreBH }\end{array}$ & $\begin{array}{l}\text { Cell cycle control, mitosis and } \\
\text { meiosis }\end{array}$ & & & 1.72 \\
\hline Imo1726 & 301 & yulF & $\begin{array}{l}\text { oxidoreductase, Gfo/ldh/MocA } \\
\text { family }\end{array}$ & $\begin{array}{l}\text { General function prediction } \\
\text { only }\end{array}$ & & & 1.56 \\
\hline Imo1743 & 306 & & $\begin{array}{l}\text { FIG00774678: hypothetical pro- } \\
\text { tein }\end{array}$ & Not in COGs & & & 1.54 \\
\hline Imo1850 & 327 & уроР & $\begin{array}{l}\text { Transcriptional regulator, MarR } \\
\text { family }\end{array}$ & Transcription & & & -1.53 \\
\hline Imo1867 & 331 & & Pyruvate, phosphate dikinase & $\begin{array}{l}\text { Carbohydrate transport and } \\
\text { metabolism }\end{array}$ & & & 1.73 \\
\hline Imo1903 & & & $\begin{array}{l}\text { bacteriocin transport accessory } \\
\text { protein }\end{array}$ & $\begin{array}{l}\text { protein turnover, chaperones; } \\
\text { Energy production and conver- } \\
\text { sion; }\end{array}$ & 1.59 & $3.1 \mathrm{E}-03$ & \\
\hline Imo1911 & 343 & dgcA & GGDEF domain protein, DGC & Signal transduction mechanisms & 1.62 & $2.0 \mathrm{E}-04$ & \\
\hline Imo1912 & 343 & dgcB & GGDEF domain protein, DGC & Signal transduction mechanisms & 1.55 & $1.2 \mathrm{E}-02$ & \\
\hline Imo1913 & 343 & ydaJ & Lmo1913 protein & Not in COGs & 1.69 & $5.5 \mathrm{E}-03$ & \\
\hline Imo2055 & 372 & ylbF & Comk regulator & Function unknown & & & 1.52 \\
\hline Imo2067 & & yxel & Choloylglycine hydrolase & Defense/virulence mechanisms & & & 1.61 \\
\hline Imo2077 & 378 & ydic & $\begin{array}{l}\text { Inactive homolog of metal-de- } \\
\text { pendent proteases, putative } \\
\text { molecular chaperone }\end{array}$ & $\begin{array}{l}\text { Posttranslational modification, } \\
\text { protein turnover, chaperones }\end{array}$ & 1.54 & 2.7E-02 & \\
\hline Imo2150 & & & $\begin{array}{l}\text { FIG00774336: hypothetical pro- } \\
\text { tein }\end{array}$ & Not in COGs & -1.71 & 4.4E-02 & \\
\hline Imo2152 & 392 & & thioredoxin, putative & $\begin{array}{l}\text { Posttranslational modification, } \\
\text { protein turnover, chaperones; } \\
\text { Energy production and conver- } \\
\text { sion; }\end{array}$ & & & 1.72 \\
\hline Imo2157 & & sepA & alkyl sulfatase & $\begin{array}{l}\text { Secondary metabolites biosyn- } \\
\text { thesis, transport and catabolism }\end{array}$ & & & 1.65 \\
\hline Imo2183 & 396 & & $\begin{array}{l}\text { Heme transporter IsdDEF, per- } \\
\text { mease component IsdF }\end{array}$ & $\begin{array}{l}\text { Inorganic ion transport and me- } \\
\text { tabolism }\end{array}$ & & & 1.9 \\
\hline Imo2184 & 396 & ygaD & $\begin{array}{l}\text { Heme transporter IsdDEF, lipo- } \\
\text { protein IsdE }\end{array}$ & $\begin{array}{l}\text { Inorganic ion transport and me- } \\
\text { tabolism }\end{array}$ & & & 1.53 \\
\hline Imo2186 & 396 & & $\begin{array}{l}\text { NPQTN cell wall anchored pro- } \\
\text { tein IsdC }\end{array}$ & Cell wall/membrane biogenesis & & & 1.61 \\
\hline Imo2199 & 398 & ohrA & $\begin{array}{l}\text { Organic hydroperoxide re- } \\
\text { sistance protein }\end{array}$ & $\begin{array}{l}\text { Posttranslational modification, } \\
\text { protein turnover, chaperones }\end{array}$ & & & 1.5 \\
\hline Imo2230 & & & arsenate reductase & Signal transduction mechanisms & & & 1.5 \\
\hline Imo2345 & 419 & ytmo & $\begin{array}{l}\text { Bacterial luciferase family pro- } \\
\text { tein YtmO, in cluster with L-cys- } \\
\text { tine } A B C \text { transporter }\end{array}$ & $\begin{array}{l}\text { Energy production and conver- } \\
\text { sion }\end{array}$ & & & 2.82 \\
\hline Imo2349 & 419 & tyck & $\begin{array}{l}\text { L-Cystine ABC transporter, } \\
\text { periplasmic cystine-binding pro- } \\
\text { tein TcyK }\end{array}$ & $\begin{array}{l}\text { Amino acid transport and me- } \\
\text { tabolism; Signal transduction } \\
\text { mechanisms; }\end{array}$ & & & 3.4 \\
\hline Imo2407 & & & $\begin{array}{l}\text { FIG00774515: hypothetical pro- } \\
\text { tein }\end{array}$ & Not in COGs & -1.72 & $7.4 \mathrm{E}-02$ & -2.72 \\
\hline
\end{tabular}


Supplementary data

\begin{tabular}{|c|c|c|c|c|c|c|c|}
\hline \multirow{2}{*}{ Locus tag } & \multirow{2}{*}{ Operon } & \multirow{2}{*}{ Name } & \multirow{2}{*}{ RAST info } & \multirow{2}{*}{ Function (COG) } & \multicolumn{2}{|c|}{$\begin{array}{c}\text { Transcriptome } \\
\Delta \text { rli31/Prli31 }\end{array}$} & \multirow{2}{*}{$\begin{array}{c}\text { Proteome } \\
\Delta r \text { rli31/Prli31 } \\
\text { Fold change } \\
\text { NS }^{1}\end{array}$} \\
\hline & & & & & $\begin{array}{c}\text { Fold } \\
\text { change }\end{array}$ & $\mathbf{p}$ & \\
\hline Imo2463 & 436 & & $\begin{array}{l}\text { FlG00774322: hypothetical pro- } \\
\text { tein }\end{array}$ & $\begin{array}{l}\text { General function prediction } \\
\text { only }\end{array}$ & & & 1.5 \\
\hline Imo2522 & & yocH & Cell wall-binding protein & $\begin{array}{l}\text { Cell wall/membrane biogenesis; } \\
\text { Function unknown; }\end{array}$ & & & -1.56 \\
\hline Imo2575 & 462 & CzcD & $\begin{array}{l}\text { Cobalt-zinc-cadmium resistance } \\
\text { protein CzcD }\end{array}$ & $\begin{array}{l}\text { Inorganic ion transport and me- } \\
\text { tabolism }\end{array}$ & 1.72 & $4.1 \mathrm{E}-02$ & \\
\hline Imo2586 & 465 & yrhE & $\begin{array}{l}\text { Formate dehydrogenase related } \\
\text { protein }\end{array}$ & $\begin{array}{l}\text { General function prediction } \\
\text { only } \\
\text { Transcription:Carbohydrate }\end{array}$ & 1.77 & $4.5 \mathrm{E}-02$ & \\
\hline Imo2652 & & $\operatorname{manR}$ & PTS system, IIA component & $\begin{array}{l}\text { Transcription; Carbohydrate } \\
\text { transport and metabolism; Sig- } \\
\text { nal transduction mechanisms; }\end{array}$ & & & 1.75 \\
\hline Imo2658 & 476 & yhxD & $\begin{array}{l}\text { Histone acetyltransferase HPA2 } \\
\text { and related acetyltransferases }\end{array}$ & $\begin{array}{l}\text { Transcription; General function } \\
\text { prediction only; }\end{array}$ & & & -1.65 \\
\hline Imo2663 & 477 & yjmD & $\begin{array}{l}\text { Galactitol-1-phosphate 5-dehy- } \\
\text { drogenase }\end{array}$ & $\begin{array}{l}\text { Amino acid transport and me- } \\
\text { tabolism; General function pre- } \\
\text { diction only; }\end{array}$ & 1.64 & $6.0 \mathrm{E}-02$ & \\
\hline Imo2667 & 477 & & $\begin{array}{l}\text { PTS system, galactitol-specific } \\
\text { IIA component }\end{array}$ & $\begin{array}{l}\text { Carbohydrate transport and } \\
\text { metabolism; Signal transduction } \\
\text { mechanisms; }\end{array}$ & 1.6 & $3.1 \mathrm{E}-02$ & 1.57 \\
\hline Imo2691 & & & $\begin{array}{l}\text { Membrane-bound lytic murein } \\
\text { transglycosylase D precursor }\end{array}$ & $\begin{array}{l}\text { Cell wall/membrane biogenesis; } \\
\text { Cell motility; Intracellular traf- } \\
\text { ficking and secretion; }\end{array}$ & & & 1.6 \\
\hline Imo2697 & 485 & & $\begin{array}{l}\text { Phosphoenolpyruvate-dihydrox- } \\
\text { yacetone phosphotransferase }\end{array}$ & Function unknown & & & 1.57 \\
\hline Imo2758 & & guaB & $\begin{array}{l}\text { Inosine-5'-monophosphate de- } \\
\text { hydrogenase }\end{array}$ & $\begin{array}{l}\text { Nucleotide transport and me- } \\
\text { tabolism; General function pre- } \\
\text { diction only; }\end{array}$ & 1.58 & $3.6 \mathrm{E}-02$ & \\
\hline Imo2805 & 508 & & hypothetical protein & Not in COGs & -2.12 & $3.6 \mathrm{E}-02$ & \\
\hline
\end{tabular}

${ }_{1}^{1}=$ changes in protein abundance are non-significant changes, ${ }^{2}=$ detected in all $\mathrm{P}_{r l i 31}{ }^{*}$ samples but no peptide was found in $\Delta r$ rli31, ${ }^{3}=$ significant and 40.34 fold downregulated in $\triangle r l i 31 / w t$, RAST= Rapid Annotation using Subsystem Technology (database), COG=Clusters of Orthologous Groups (database),

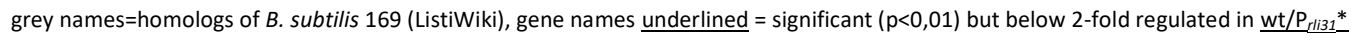


Cyclic di-AMP and osmoregulation in Listeria monocytogenes 


\section{References}

Andersson, D. I., Hughes. D. (2009) Gene amplification and adaptive evolution in bacteria. Annu. Rev. Genet. 43, 167-195.

Archer, K. A., Durack, J., Portnoy, D. A. (2014) STING-dependent type I IFN production inhibits cell-mediated immunity to Listeria monocytogenes. PLoS Pathog. 10, e1003861.

Arnaud, M., Chastanet, A., Débarbouillé, M. (2004) New vector for efficient allelic replacement in naturally non-transformable, low-GC-content, Gram-positive bacteria. Appl. Environ. Microbiol. 70, 6887-6891.

Aziz, R. K., Bartels, D., Best, A. A., DeJongh, M., Disz, T., Edwards, R. A., Formsma, K., Gerdes, S., Glass, E. M., Kubal, M., et al. (2008) The RAST Server: Rapid Annotations using Subsystems Technology. BMC Genomics. 9, 75.

Bai, Y., Yang, J., Zarrella, T. M., Zhang, Y., Metzger, D. W., Bai, G. (2014) Cyclic di-AMP impairs potassium uptake mediated by a cyclic di-AMP binding protein in Streptococcus pneumoniae. J. Bacteriol. 196, 614-623.

Bai, Y., Yang, J., Zhou, X., Ding, X., Eisele, L. E., Bai, G. (2012) Mycobacterium tuberculosis Rv3586 (DacA) is a diadenylate cyclase that converts ATP or ADP into c-di-AMP. PLoS one. 7, e35206.

Barb, A. W., Cort, J. R., Seetharaman, J., Lew, S., Lee, H. W., Acton, T., Xiao, R., Kennedy, M. A., Tong, L., Montelione, G. T., et al. (2010) Structures of domains I and IV from YbbR are representative of a widely distributed protein family. Protein Sci. 20, 396-405.

Barreteau, H., Kovac, A., Boniface, A., Sova, M., Gobec, S., Blanot, D. (2008) Cytoplasmic steps of peptidoglycan biosynthesis. FEMS Microbiol. Rev. 32, 168-207.

Barrick, J. E., Lenski, R. E. (2013) Genome dynamics during experimental evolution. Nat. Rev. Genet. 14, 827-839.

Bécavin, C., Koutero, M., Tchitchek, N., Cerutti, F., Lechat, P., Maillet, N., Hoede, C., Chiapello, H., Gaspin, C., Cossart, P. (2017) Listeriomics: an interactive web platform for systems biology of Listeria. mSystems. 2, e00186-16.

Bejerano-Sagie, M., Oppenheimer-Shaanan, Y., Berlatzky, I., Rouvinski, A., Meyerovich, M., Ben-Yehuda, S. (2006) A checkpoint protein that scans the chromosome for damage at the start of sporulation in Bacillus subtilis. Cell. 125, 679-690.

Ben-Yehuda, S., Rudner, D. Z., Losick, R. (2003) RacA, a bacterial protein that anchors chromosomes to the cell poles. Science. 299, 532-536.

Bi, W., Stambrook, P. J. (1997) CCR: a rapid and simple approach for mutation detection. Nucleic Acids Res. 25, 2949-2951.

Blötz, C., Treffon, K., Kaever, V., Schwede, F., Hammer, E., Stülke, J. (2017) Identification of the components involved in cyclic di-AMP signaling in Mycoplasma pneumoniae. Front. Microbiol. 8, 1328.

Bochner, B. R., Gadzinski, P., Panomitros, E. (2001) Phenotype MicroArrays for high-throughput phenotypic testing and assay of gene function. Genome Res. 11, 1246-1255. 
Boura, M., Keating, C., Royet, K., Paudyal, R., O'Donoghue, B., O'Byrne, C. P., Karatzas, K. (2016) Loss of SigB in Listeria monocytogenes strains EGD-e and 10403S confers hyperresistance to hydrogen peroxide in stationary phase under aerobic conditions. Appl. Environ. Microbiol. 82, 4584-4591.

Bowman, L., Zeden, M. S., Schuster, C. F., Kaever, V., Gründling, A. (2016) New insights into the cyclic diadenosine monophosphate (c-di-AMP) degradation pathway and the requirement of the cyclic dinucleotide for acid stress resistance in Staphylococcus aureus. J. Biol. Chem. 291, 26970-26986.

Burguière, P., Fert, J., Guillouard, I., Auger, S., Danchin, A., Martin-Verstraete, I. (2005) Regulation of the Bacillus subtilis ytml operon, involved in sulfur metabolism. J. Bacteriol. 187, 6019-6030.

Burke, T. P. (2018) The unexpected effects of the combination of antibiotics and immunity. Cell. 172, 891-893.

Burke, T. P., Loukitcheva, A., Zemansky, J., Wheeler, R., Boneca, I. G., Portnoy, D. A. (2014) Listeria monocytogenes is resistant to lysozyme through the regulation, not the acquisition, of cell wall-modifying enzymes. J. Bacteriol. 196, 3756-3767.

Burke, T. P., Portnoy, D. A. (2016) SpoVG is a conserved RNA-binding protein that regulates Listeria monocytogenes lysozyme resistance, virulence, and swarming motility. mBio. 7, e00240.

Cagliero, C., Jin, D. J. (2013) Dissociation and re-association of RNA polymerase with DNA during osmotic stress response in Escherichia coli. Nucleic Acids Res. 41, 315-326.

Callewaert, L., Michiels, C. W. (2010) Lysozymes in the animal kingdom. J. Biosci. 35, 127-160.

Campeotto, I., Zhang, Y., Mladenov, M. G., Freemont, P. S., Gründling, A. (2015) Complex structure and biochemical characterization of the Staphylococcus aureus cyclic diadenylate monophosphate (c-di-AMP)-binding protein PstA, the founding member of a new signal transduction protein family. J. Biol. Chem. 290, 2888-2901.

Campos, S. S., Ibarra-Rodriguez, J. R., Barajas-Ornelas, R. C., Ramírez-Guadiana, F. H., ObregónHerrera, A., Setlow, P., Pedraza-Reyes, M. (2014) Interaction of apurinic/apyrimidinic endonucleases Nfo and ExoA with the DNA integrity scanning protein DisA in the processing of oxidative DNA damage during Bacillus subtilis spore outgrowth. J. Bacteriol. 196, 568-578.

Cascante-Estepa, N., Gunka, K., Stülke, J. (2016) Localization of components of the RNA-degrading machine in Bacillus subtilis. Front. Microbiol. 7, 1492.

Chen, L. H., Köseoğlu, V. K., Güvener, Z. T., Myers-Morales, T., Reed, J. M., D'Orazio, S. E., Miller, K. W., Gomelsky, M. (2014) Cyclic di-GMP-dependent signaling pathways in the pathogenic Firmicute Listeria monocytogenes. PLoS Pathog. 10, e1004301.

Cheng, C., Wang, H., Ma, T., Han, X., Yang, Y., Sun, J., Chen, Z., Yu, H., Hang, Y., Liu, F., et al. (2018) Flagellar basal body structural proteins FlhB, FliM, and FliY are required for flagellarassociated protein expression in Listeria monocytogenes. Front. Microbiol. 9, 208.

Chico-Calero, I., Suárez, M., González-Zorn, B., Scortti, M., Slaghuis, J., Goebel, W., VázquezBoland, J. A., European Listeria Genome Consortium. (2002) Hpt, a bacterial homolog of the microsomal glucose-6-phosphate translocase, mediates rapid intracellular proliferation in Listeria. Proc. Natl. Acad. Sci. U.S.A. 99, 431-436.

Chin, K. H., Liang, J. M., Yang, J. G., Shih, M. S., Tu, Z. L., Wang, Y. C., Sun, X. H., Hu, N. J., Liang, Z. X., Dow, J. M., et al. (2015) Structural insights into the distinct binding mode of cyclic diAMP with SaCpaA-RCK. Biochemistry. 54, 4936-4951. 
Choi, P. H., Sureka, K., Woodward, J. J., Tong, L. (2015) Molecular basis for the recognition of cyclic-di-AMP by PstA, a PII-like signal transduction protein. Microbiologyopen. 4, 361-374.

Choi, P. H., Vu, T. M. N., Pham, H. T., Woodward, J. J., Turner, M. S., Tong, L. (2017) Structural and functional studies of pyruvate carboxylase regulation by cyclic di-AMP in lactic acid bacteria. Proc. Natl. Acad. Sci. USA. 114, E7226-E7235.

Claessen, D., Emmins, R., Hamoen, L. W., Daniel, R. A., Errington, J., Edwards, D. H. (2008) Control of the cell elongation-division cycle by shuttling of PBP1 protein in Bacillus subtilis. Mol. Microbiol. 68, 1029-1046.

Clarke, C. A., Scheurwater, E. M., Clarke, A. J. (2010) The vertebrate lysozyme inhibitor Ivy functions to inhibit the activity of lytic transglycosylase. J. Biol. Chem. 285, 14843-14847.

Clemens, R., Zaschke-Kriesche, J., Khosa, S., Smits, S. (2018) Insight into two ABC transporter families involved in lantibiotic resistance. Front. Mol. Biosci. 4, 91.

Commichau, F. M., Dickmanns, A., Gundlach, J., Ficner, R., Stülke, J. (2015) A jack of all trades: the multiple roles of the unique essential second messenger cyclic di-AMP. Mol. Microbiol. 97, 189-204.

Commichau, F. M., Gibhardt, J., Halbedel, S., Gundlach, J., Stülke, J. (2018) A delicate connection: c-di-AMP affects cell Integrity by controlling osmolyte transport. Trends Microbiol. 26, 175-185.

Commichau, F. M., Heidemann, J. L., Ficner, R., Stülke, J. (2019) Making and breaking of an essential poison: the cyclases and phosphodiesterases that produce and degrade the essential second messenger cyclic di-AMP in bacteria. J. Bacteriol. 201, e00462-18.

Commichau, F. M., Rothe, F. M., Herzberg, C., Wagner, E., Hellwig, D., Lehnik-Habrink, M., Hammer, E., Völker, U., Stülke, J. (2009) Novel activities of glycolytic enzymes in Bacillus subtilis: interactions with essential proteins involved in mRNA processing. Mol. Cell Proteom. 8, 1350-1360.

Commichau, F. M., Stülke, J. (2008) Trigger enzymes: bifunctional proteins active in metabolism and in controlling gene expression. Mol. Microbiol. 67, 692-702.

Corrigan, R. M., Abbott, J. C., Burhenne, H., Kaever, V., Gründling, A. (2011) c-di-AMP is a new second messenger in Staphylococcus aureus with a role in controlling cell size and envelope stress. PLoS Pathog. 7, e1002217.

Corrigan, R. M., Bowman, L., Willis, A. R., Kaever, V., Gründling, A. (2015) Cross-talk between two nucleotide-signaling pathways in Staphylococcus aureus. J. Biol. Chem. 290, 5826-5839.

Corrigan, R. M., Campeotto, I., Jeganathan, T., Roelofs, K. G., Lee, V. T., Gründling, A. (2013) Systematic identification of conserved bacterial c-di-AMP receptor proteins. Proc. Natl. Acad. Sci. U.S.A. 110, 9084-9089.

Corrigan, R. M., Gründling, A. (2013) Cyclic di-AMP: another second messenger enters the fray. Nat. Rev. Microbiol. 11, 513-524.

Dar, D., Shamir, M., Mellin, J. R., Koutero, M., Stern-Ginossar, N., Cossart, P., Sorek, R. (2016) Term-seq reveals abundant ribo-regulation of antibiotics resistance in bacteria. Science. 352, aad9822.

de las Heras, A., Cain, R. J., Bielecka, M. K., Vázquez-Boland, J. A. (2011) Regulation of Listeria virulence: PrfA master and commander. Curr. Opin. Microbiol. 14, 118-127. 
Dengler, V., McCallum, N., Kiefer, P., Christen, P., Patrignani, A., Vorholt, J. A., Berger-Bächi, B., Senn, M. M. (2013) Mutation in the c-di-AMP cyclase dacA affects fitness and resistance of methicillin resistant Staphylococcus aureus. PLoS one. 8, e73512.

Devaux, L., Sleiman, D., Mazzuoli, M.-V., Gominet, M., Lanotte, P., Trieu-Cuot, P., Kaminski, P.A., Firon, A. (2018) Cyclic di-AMP regulation of osmotic homeostasis is essential in Group B Streptococcus. PLoS Genet. 14, e1007342.

Dons, L., Rasmussen, O. F., Olsen, J.E. (1992) Cloning and characterization of a gene encoding flagellin of Listeria monocytogenes. Mol. Microbiol. 6, 2919-2929.

Dussurget, O., Cabanes, D., Dehoux, P., Lecuit, M., Buchrieser, C., Glaser, P., Cossart, P. (2002) Listeria monocytogenes bile salt hydrolase is a PrfA-regulated virulence factor involved in the intestinal and hepatic phases of listeriosis. Mol. Microbiol. 45, 1095-1106.

Elbakush, A. M., Miller, K. W., Gomelsky, M. (2018) CodY-mediated c-di-GMP-dependent inhibition of mammalian cell invasion in Listeria monocytogenes. J. Bacteriol. 200, e00457-17.

Even, S., Burguière, P., Auger, S., Soutourina, O., Danchin, A., Martin-Verstraete, I. (2006) Global control of cysteine metabolism by CymR in Bacillus subtilis. J. Bacteriol. 188, 21842197.

Farber, J. M., Peterkin, P. I. (1991) Listeria monocytogenes, a food-borne pathogen. Microbiol. Rev. 55, 476-511.

Fedorov, R., Meshcheryakov, V., Gongadze, G., Fomenkova, N., Nevskaya, N., Selmer, M., Laurberg, M., Kristensen, O., Al-Karadaghi, S., Liljas, A., et al. (2001) Structure of ribosomal protein TL5 complexed with RNA provides new insights into the CTC family of stress proteins. Acta Crystallogr. D. 57, 968-976.

Feehily, C., O'Byrne, C. P., Karatzas, K. A. (2013) Functional y-aminobutyrate shunt in Listeria monocytogenes: role in acid tolerance and succinate biosynthesis. Appl. Environ. Microbiol. 79, 74-80.

Foster, A. W., Osman, D., Robinson, N. J. (2014) Metal preferences and metallation. J.Biol. Chem. 289, 28095-29103.

Freitag, N. E., Port, G. C., Miner, M. D. (2009) Listeria monocytogenes - from saprophyte to intracellular pathogen. Nat. Rev. Microbiol. 7, 623-628.

Fritsch, F., Mauder, N., Williams, T., Weiser, J., Oberle, M., Beier, D. (2011) The cell envelope stress response mediated by the LiaFSR $R_{L m}$ three-component system of Listeria monocytogenes is controlled via the phosphatase activity of the bifunctional histidine kinase $\mathrm{LiaS}_{\mathrm{Lm}}$. Microbiology. 157, 373-386.

Gándara, C., Alonso, J. C. (2015) DisA and c-di-AMP act at the intersection between DNA-damage response and stress homeostasis in exponentially growing Bacillus subtilis cells. DNA Repair (Amst.). 27, 1-8.

Gándara, C., de Lucena, D. K. C., Torres, R., Serrano, E., Altenburger, S., Graumann, P. L., Alonso, J. C. (2017) Activity and in vivo dynamics of Bacillus subtilis DisA are affected by RadA/Sms and by holliday junction-processing proteins. DNA Repair (Amst.). 55, 17-30.

Gao, A., Serganov, A. (2014) Structural insights into recognition of c-di-AMP by the ydaO riboswitch. Nat. Chem. Biol. 10, 787-792.

Gao, X., Mukherjee, S., Matthews, P. M., Hammad, L. A., Kearns, D. B., Dann, C. E. (2013) Functional characterization of core components of the Bacillus subtilis cyclic-di-GMP signaling pathway. J. Bacteriol. 195, 4782-4792. 
Gardan, R., Cossart, P., Labadie, J., European Listeria Genome Consortium. (2003) Identification of Listeria monocytogenes genes involved in salt and alkaline-pH tolerance. Appl. Environ. Microbiol. 69, 3137-3143.

Gay, P., Le Coq, D., Steinmetz, M., Ferrari, E., Hoch, J. A. (1983) Cloning structural gene sacB, which codes for exoenzyme levansucrase of Bacillus subtilis: expression of the gene in Escherichia coli. J. Bacteriol. 153, 1424-1431.

Geiger, T., Wolz, C. (2014) Intersection of the stringent response and the CodY regulon in low GC Gram-positive bacteria. Int. J. Med. Microbiol. 304, 150-155.

Gertz, S., Engelmann, S., Schmid, R., Ohlsen, K., Hacker, J., Hecker, M. (1999) Regulation of $\sigma^{B}-$ dependent transcription of $\operatorname{sig} B$ and asp23 in two different Staphylococcus aureus strains. Mol. Gen. Genet. 261, 558-566.

Gibhardt, J. (2015) Cyclic di-AMP metabolism and osmoregulation in Listeria monocytogenes. Master's thesis. Georg-August-Universität Göttingen.

Glaser, P., Frangeul, L., Buchrieser, C., Rusniok, C., Amend, A., Baquero, F., Berche, P., Bloecker, H., Brandt, P., Chakraborty, T. et al. (2001) Comparative genomics of Listeria species. Science. 294, 849-852.

Gomelsky M. (2011) cAMP, c-di-GMP, c-di-AMP and now cGMP: bacteria use them all!. Mol. Microbiol. 79, 562-565.

Gray, M. L., Killinger, A. H. (1966) Listeria monocytogenes and listeric infections. Bacteriol. Rev. 30, 309-382.

Gray, W. T., Govers, S. K., Xiang, Y., Parry, B. R., Campos, M., Kim, S., Jacobs-Wagner, C. (2018) Nucleoid size scaling and intracellular organization of translation across-bacteria. bioRxiv. 479840.

Groisman, E. A., Hollands, K., Kriner, M. A., Lee, E. J., Park, S. Y., Pontes, M. H. (2013) Bacterial $\mathrm{Mg}^{2+}$ homeostasis, transport, and virulence. Annu. Rev. Genet. 47, 625-646.

Gründling, A. (2013) Potassium uptake systems in Staphylococcus aureus: new stories about ancient systems. mBio. 4, e00784-13.

Gründling, A., Burrack, L. S., Bouwer, H. G., Higgins, D. E. (2004) Listeria monocytogenes regulates flagellar motility gene expression through $\operatorname{MogR}$, a transcriptional repressor required for virulence. Proc. NatI. Acad. Sci. U.S.A. 101, 12318-12323.

Gueriri, I., Cyncynatus, C., Dubrac, S., Arana, A. T., Dussurget, O., Msadek, T. (2008) The DegU orphan response regulator of Listeria monocytogenes autorepresses its own synthesis and is required for bacterial motility, virulence and biofilm formation. Microbiology. 154, 22512264.

Guérout-Fleury, A. M., Shazand, K., Frandsen, N., Stragier, P. (1995) Antibiotic-resistance cassettes for Bacillus subtilis. Gene. 167, 335-336.

Gundlach, J., Dickmanns, A., Schröder-Tittmann, K., Neumann, P., Kaesler, J., Kampf, J., Herzberg, C., Hammer, E., Schwede, F., Kaever, V., et al. (2015) Identification, characterization, and structure analysis of the cyclic di-AMP-binding PII-like signal transduction protein DarA. J. Biol. Chem. 290, 3069-3080.

Gundlach, J., et al. (2019) unpublished.

Gundlach, J., Herzberg, C., Kaever, V., Gunka, K., Hoffmann, T., Weiß, M., Gibhardt, J., Thürmer, A., Hertel, D., Daniel, R., et al. (2017) Control of potassium homeostasis is an essential function of the second messenger cyclic di-AMP in Bacillus subtilis. Sci. Signal. 10, eaal3011. 
Gundlach, J., Mehne, F. M., Herzberg, C., Kampf, J., Valerius, O., Kaever, V., Stülke, J. (2015) An essential poison: synthesis and degradation of cyclic di-AMP in Bacillus subtilis. J. Bacteriol. 197, 3265-3274.

Gundlach, J., Rath, H., Herzberg, C., Mäder, U., Stülke, J. (2016) Second messenger signaling in Bacillus subtilis: accumulation of cyclic di-AMP inhibits biofilm formation. Front. Microbiol. 7, 804.

Guzman, L. M., Belin, D., Carson, M. J., Beckwith, J. (1995) Tight regulation, modulation, and high-level expression by vectors containing the arabinose $\mathrm{P}_{\mathrm{BAD}}$ promoter. J. Bacteriol. 177, 4121-4130.

Halbedel, S., Hahn, B., Daniel, R. A., Flieger, A. (2012) DivIVA affects secretion of virulence-related autolysins in Listeria monocytogenes. Mol. Microbiol. 83, 821-839.

Hauf, S., Herrmann, J., Miethke, M., Gibhardt, J., Commichau, F. M., Müller, R., Fuchs, S., Halbedel, S. (2019) Aurantimycin resistance genes contribute to survival of Listeria monocytogenes during life in the environment. Mol. Microbiol. doi: 10.1111/mmi.14205.

Hengge, R. (2009) Principles of c-di-GMP signaling in bacteria. Nat. Rev. Micribiol. 7, 263-273.

Hengge, R., Gründling, A., Jenal, U., Ryan, R., Yildiz, F. (2015) Bacterial signal transduction by cyclic di-GMP and other nucleotide second messengers. J. Bacteriol. 198, 15-26.

Hoffmann, T., Boiangiu, C., Moses, S., Bremer, E. (2008) Responses of Bacillus subtilis to hypotonic challenges: physiological contributions of mechanosensitive channels to cellular survival. Appl. Environ. Microbiol. 74, 2454-2460.

Holtmann, G., Bakker, E. P., Uozumi, N., Bremer, E. (2003) KtrAB and KtrCD: two K ${ }^{+}$uptake systems in Bacillus subtilis and their role in adaptation to hypertonicity. J. Bacteriol. 185, 12891298.

Horak, J., Wolf, D. H. (1997) Catabolite inactivation of the galactose transporter in the yeast Saccharomyces cerevisiae: ubiquitination, endocytosis, and degradation in the vacuole. J. Bacteriol. 179, 1541-1549.

Horton, R. M., Cai, Z. L., Ho, S. N., Pease, L. R. (1990) Gene splicing by overlap extension: tailormade genes using the polymerase chain reaction. Biotechniques. 8, 528-535.

Huynh, T. N., Choi, P. H., Sureka, K., Ledvina, H. E., Campillo, J., Tong, L., Woodward, J. J. (2016) Cyclic di-AMP targets the cystathionine beta-synthase domain of the osmolyte transporter OpuC. Mol. Microbiol. 102, 233-243.

Huynh, T. N., Luo, S., Pensinger, D., Sauer, J. D., Tong, L., Woodward, J. J. (2015) An HD-domain phosphodiesterase mediates cooperative hydrolysis of c-di-AMP to affect bacterial growth and virulence. Proc. Natl. Acad. Sci. U.S.A. 112, E747-E756.

Huynh, T. N., Woodward, J. J. (2016) Too much of a good thing: regulated depletion of c-di-AMP in the bacterial cytoplasm. Curr. Opin. Microbiol. 30, 22-29.

Kalia, D., Merey, G., Nakayama, S., Zheng, Y., Zhou, J., Luo, Y., Guo, M., Roembke, B. T., Sintim, H. O. (2013) Nucleotide, c-di-GMP, c-di-AMP, cGMP, cAMP, (p)ppGpp signaling in bacteria and implications in pathogenesis. Chem. Soc. Rev. 42, 305-341.

Kamp, H. D., Higgins, D. E. (2009) Transcriptional and post-transcriptional regulation of the GmaR antirepressor governs temperature-dependent control of flagellar motility in Listeria monocytogenes. Mol. Microbiol. 74, 421-425.

Kamp, H. D., Higgins, D. E. (2011) A protein thermometer controls temperature-dependent transcription of flagellar motility genes in Listeria monocytogenes. PLoS Pathog. 7, e1002153. 
Kaplan Zeevi, M., Shafir, N. S., Shaham, S., Friedman, S., Sigal, N., Nir Paz, R., Boneca, I. G., Herskovits, A. A. (2013) Listeria monocytogenes multidrug resistance transporters and cyclic di-AMP, which contribute to type I interferon induction, play a role in cell wall stress. J. Bacteriol. 195, 5250-5261.

Karimova, G., Pidoux, J., Ullmann, A., Ladant, D. (1998) A bacterial two-hybrid system based on a reconstituted signal transduction pathway. Proc. Natl. Acad. Sci. U.S.A. 95, 5752-5756.

Karimova, G., Robichon, C., Ladant, D. (2009) Characterization of YmgF, a 72-residue inner membrane protein that associates with the Escherichia coli cell division machinery. J. Bacteriol. 191, 333-346.

Kawai, Y., Mickiewicz, K., Errington, J. (2018) Lysozyme counteracts $\beta$-lactam antibiotics by promoting the emergence of L-form bacteria. Cell. 172, 1038-1049.e10.

Kearse, M., Moah, R., Wilson, A., Stones-Havas, S., Cheung, M., Sturrock, S. Buxton, S., Cooper, A., Markowitz, S., Duran, C., et al. (2012) Geneious basic: an integrated and extendable desktop software platform for the organization and analysis of sequence data. Bioinformatics 28, 1647-1649.

Kellenberger, C. A., Chen, C., Whiteley, A. T., Portnoy, D. A., Hammond, M. C. (2015) RNA-based fluorescent biosensors for live cell imaging of second messenger cyclic di-AMP. J. Am. Chem. Soc. 137, 6432-6435.

Kempf, B., Bremer, E. (1998) Stress responses of Bacillus subtilis to high osmolarity environments: uptake and synthesis of osmoprotectants. J. Biosci. 23, 447-455.

Kersey, P. J., Allen, J. E., Allot, A., Barba, M., Boddu, S., Bolt, B. J., Carvalho-Silva, D., Christensen, M., Davis, P., Grabmueller, C., et al. (2017) Ensembl Genomes 2018: an integrated omics infrastructure for non-vertebrate species. Nucleic Acids Res. 46, D802-D808.

Kim, H., Youn, S. J., Kim, S. O., Ko, J., Lee, J. O., Choi, B. S. (2015) Structural studies of potassium transport protein KtrA regulator of conductance of $\mathrm{K}^{+}(\mathrm{RCK}) \mathrm{C}$ domain in complex with cyclic diadenosine monophosphate (c-di-AMP). J. Biol. Chem. 290, 16393-16402.

Ko, R., Smith, L. T., Smith, G. M. (1994) Glycine betaine confers enhanced osmotolerance and cryotolerance on Listeria monocytogenes. J. Bacteriol. 176, 426-431.

Korolev, N., Lyubartsev, A. P., Rupprecht, A., Nordenskiöld, L. (1999) Competitive binding of $\mathrm{Mg}^{2+}, \mathrm{Ca}^{2+}, \mathrm{Na}^{+}$, and $\mathrm{K}^{+}$ions to DNA in oriented DNA fibers: experimental and Monte Carlo simulation results. Biophys. J. 77, 2736-2749.

Köseoğlu, V. K., Heiss, C., Azadi, P., Topchiy, E., Güvener, Z. T., Lehmann, T. E., Miller, K. W., Gomelsky, M. (2015) Listeria monocytogenes exopolysaccharide: origin, structure, biosynthetic machinery and c-di-GMP-dependent regulation. Mol. Microbiol. 96, 728-743.

Nollet, J. (1748) Recherches sur les causes du bouillonnement des liquides. In: Mémoires de Mathématique et de Physique, tirés des registres de l'Académie Royale des Sciences de I'année 1748. 57-104.

Laxminarayan, R., Duse, A., Wattal, C., Zaidi, A. K. M., Wertheim, H. F. L., Sumpradit, N., Vlieghe, E., Hara, G. L., Gould, I. M., Goossens, H., et al. (2013) Antibiotic resistance-the need for global solutions. Lancet. Infect. Dis. 13, 1057-1098.

Lima, A., Durán, R., Schujman, G. E., Marchissio, M. J., Portela, M. M., Obal, G., Pritsch, O., de Mendoza, D., Cerveñansky, C. (2011) Serine/threonine protein kinase PrkA of the human pathogen Listeria monocytogenes: biochemical characterization and identification of interacting partners through proteomic approaches. J. Proteom. 74, 1720-1734. 
Lobel, L., Herskovits, A. A. (2016) Systems level analyses reveal multiple regulatory activities of CodY controlling metabolism, motility and virulence in Listeria monocytogenes. PLoS Genet. 12, e1005870.

Lobel, L., Sigal, N., Borovok, I., Belitsky, B. R., Sonenshein, A. L., Herskovits, A. A. (2015) The metabolic regulator CodY links Listeria monocytogenes metabolism to virulence by directly activating the virulence regulatory gene prfA. Mol. Microbiol. 95, 624-644.

López-Maury, L., Marguerat, S., Bähler, J. (2008) Tuning gene expression to changing environments: from rapid responses to evolutionary adaptation. Nat. Rev. Genet. 9, 583-593.

Low, J. C., Donachie, W. (1997) A review of Listeria monocytogenes and listeriosis. Vet. J. 153, 9-29.

Lu, M., Steitz, T. A. (2000) Structure of Escherichia coli ribosomal protein L25 complexed with a 5S rRNA fragment at 1.8-Å resolution. Proc. Natl. Acad. Sci. U.S.A. 97, 2023-2038.

Luo, Y., Helmann, J. D. (2012) Analysis of the role of Bacillus subtilis $\sigma^{\mathrm{M}}$ in $\beta$-lactam resistance reveals an essential role for c-di-AMP in peptidoglycan homeostasis. Mol. Microbiol. 83, 623639.

Manikandan, K., Sabareesh, V., Singh, N., Saigal, K., Mechold, U., Sinha, K. M. (2014) Two-step synthesis and hydrolysis of cyclic di-AMP in Mycobacterium tuberculosis. PLoS one. 9, e86096.

Manson, M. D., Tedesco, P., Berg, H. C., Harold, F. M., van der Drift, C. (1977) A protonmotive force drives bacterial flagella. Proc. Natl. Acad. Sci. U.S.A. 74, 3060-3064.

Manuse, S., Fleurie, A., Zucchini, L., Lesterlin, C., Grangeasse, C. (2016) Role of eukaryotic-like serine/threonine kinases in bacterial cell division and morphogenesis. FEMS Microbiol. Rev. 40, 41-56.

Martínez-Antonio, A., Medina-Rivera, A., Collado-Vides, J. (2009) Structural and functional map of a bacterial nucleoid. Genome Biol. 10, 247.

Mauder, N., Williams, T., Fritsch, F., Kuhn, M., Beier, D. (2008) Response regulator DegU of Listeria monocytogenes controls temperature-responsive flagellar gene expression in its unphosphorylated state. J. Bacteriol. 190, 4777-4781.

McFarland, A. P., Burke, T. P., Carletti, A. A., Glover, R. C., Tabakh, H., Welch, M. D., Woodward, J. J. (2018) RECON-dependent inflammation in hepatocytes enhances Listeria monocytogenes cell-to-cell spread. mBio. 9, e00526-18.

McFarland, A. P., Luo, S., Ahmed-Qadri, F., Zuck, M., Thayer, E. F., Goo, Y. A., Hybiske, K., Tong, L., Woodward, J. J. (2017) Sensing of bacterial cyclic dinucleotides by the oxidoreductase RECON promotes NF-KB activation and shapes a proinflammatory antibacterial state. Immunity. 46, 433-445.

Mehne, F. M. P. (2014) Bildung und Homöostase von c-di-AMP in Bacillus subtilis. Dissertation. Georg-August-Universität Göttingen. http://hdl.handle.net/11858/00-1735-0000-00225F16-8, accessed 03.02.2019.

Mehne, F. M. P., Gunka, K., Eilers, H., Herzberg, C., Kaever, V., Stülke, J. (2013) Cyclic di-AMP homeostasis in Bacillus subtilis: both lack and high level accumulation of the nucleotide are detrimental for cell growth. J. Biol. Chem. 288, 2004-2017.

Mehne, F. M., Schröder-Tittmann, K., Eijlander, R. T., Herzberg, C., Hewitt, L., Kaever, V., Lewis, R. J., Kuipers, O. P., Tittmann, K., Stülke, J. (2014) Control of the diadenylate cyclase CdaS in Bacillus subtilis: an autoinhibitory domain limits cyclic di-AMP production. J. Biol. Chem. 289, 21098-21107. 
Mellin, J. R., Cossart, P. (2012) The non-coding RNA world of the bacterial pathogen Listeria monocytogenes. RNA Biol. 9, 372-378.

Mengaud, J., Dramsi, S., Gouin, E., Vazquez-Boland, J. A., Milon, G., Cossart, P. (1991) Pleiotropic control of Listeria monocytogenes virulence factors by a gene that is autoregulated. Mol. Microbiol. 5, 2273-2283.

Mengin-Lecreulx, D., van Heijenoort, J. (1996) Characterization of the essential gene glmM encoding phosphoglucosamine mutase in Escherichia coli. J. Biol. Chem. 271, 32-39.

Merzbacher, M., Detsch, C., Hillen, W., Stülke, J. (2004) Mycoplasma pneumoniae HPr kinase/phosphorylase. Eur. J. Biochem. 271, 367-374.

Meury, J., Kohiyama, M. (1992) Potassium ions and changes in bacterial DNA supercoiling under osmotic stress. FEMS Microbiol. Lett. 99, 159-164.

Misra, G., Rojas, E. R., Gopinathan, A., Huang, K. C. (2013) Mechanical consequences of cellwall turnover in the elongation of a Gram-positive bacterium. Biophys. J. 104, 2342-2352.

Monchois, V., Abergel, C., Sturgis, J., Jeudy, S., Claverie, J. M. (2001) Escherichia coli ykfE ORFan gene encodes a potent inhibitor of c-type lysozyme. J. Biol. Chem. 276, 18437-18441.

Monk, I. R., Gahan, C. G., Hill, C. (2008) Tools for functional postgenomic analysis of Listeria monocytogenes. Appl. Environ. Microbiol. 74, 3921-3934.

Moscoso, J. A., Schramke, H., Zhang, Y., Tosi, T., Dehbi, A., Jung, K., Gründling, A. (2016) Binding of cyclic di-AMP to the Staphylococcus aureus sensor kinase KdpD occurs via the universal stress protein domain and downregulates the expression of the Kdp potassium transporter. J. Bacteriol. 198, 98-110.

Mukherjee, S., Kearns, D. B. (2014) The structure and regulation of flagella in Bacillus subtilis. Annu. Rev. Genet. 48, 319-340.

Müller, M., Deimling, T., Hopfner, K. P., Witte, G. (2015) c-di-AMP recognition by Staphylococcus aureus PstA. FEBS Lett. 589, 45-51.

Müller, M., Deimling, T., Hopfner, K. P., Witte, G. (2015) Structural analysis of the diadenylate cyclase reaction of DNA-integrity scanning protein A (DisA) and its inhibition by 3'-dATP. Biochem. J. 469, 367-374.

Munita, J. M., Arias, C. A. (2016) Mechanisms of antibiotic resistance. Microbiol. Spectr. 4, VMBF-0016-2015.

Murray, E. G. D., Webb, R. A., Swann, M. B. R. (1926) A disease of rabbits characterised by a large mononuclear leucocytosis, caused by a hitherto undescribed Bacillus Bacterium monocytogenes (n.sp.). J. Pathol. Bacteriol. 29, 407-439.

Nelson, J. W., Sudarsan, N., Furukawa, K., Weinberg, Z., Wang, J. X., Breaker, R. R. (2013) Riboswitches in eubacteria sense the second messenger c-di-AMP. Nat. Chem. Biol. 9, 834-839.

Newton, A. C., Bootman, M. D., Scott, J. D. (2016) Second messengers. Cold Spring Harb Perspect Biol. 8, a005926.

Nicolas, P., Maeder, U., Dervyn, E., Rochat, T., Leduc, A., Pigeonneau, N., Bidnenko, E., Marchadier, E., Hoebeke, M., Aymerich, S., et al. (2012) Condition-dependent transcriptome reveals high-level regulatory architecture in Bacillus subtilis. Science. 335, 1103-1106.

Nierhaus K. H. (2014) $\mathrm{Mg}^{2+}, \mathrm{K}^{+}$, and the ribosome. J. Bacteriol. 196, 3817-3819.

Omasits, U., Ahrens, C. H., Müller, S., Wollscheid, B. (2014) Protter: interactive protein feature visualization and integration with experimental proteomic data. Bioinformatics. 30, 884-886. 
Oppenheimer-Shaanan, Y., Wexselblatt, E., Katzhendler, J., Yavin, E., and Ben-Yehuda, S. (2011) c-di-AMP reports DNA integrity during sporulation in Bacillus subtilis. EMBO Rep. 12, 594-601.

Pasi, M., Maddocks, J. H., Lavery, R. (2015) Analyzing ion distributions around DNA: sequencedependence of potassium ion distributions from microsecond molecular dynamics. Nucleic Acids Res. 43, 2412-2423.

Patro, R., Duggal, G., Love, M. I., Irizarry, R. A., Kingsford, C. (2017) Salmon provides fast and bias-aware quantification of transcript expression. Nat. Methods. 14, 417-419.

Peel, M., Donachie, W., Shaw, A. (1988) Temperature-dependent expression of flagella of Listeria monocytogenes studied by electron microscopy, SDS-PAGE and western blotting. J. Gen. Microbiol. 134, 2171-2178.

Pesavento, C., Becker, G., Sommerfeldt, N., Possling, A., Tschowri, N., Mehlis, A., Hengge, R. (2008) Inverse regulatory coordination of motility and curli-mediated adhesion in Escherichia coli. Genes Dev., 22, 2434-2446.

Pham, H. T., Nhiep, N. T. H., Vu, T. N. M., Huynh, T. N., Zhu, Y., Huynh, A. L. D., Chakrabortti, A., Marcellin, E., Lo, R., Howard, C. B., et al. (2018) Enhanced uptake and potassium or glycine betaine or export of cyclic-di-AMP restores osmoresistance in a high cyclic-di-AMP Lactococcus lactis mutant. PLoS Genet. 14, e1007574.

Pirie, J. H. H. (1940) Listeria: change of Name for a Genus Bacteria. Nature. 145, 164.

Poolman, B., Spitzer, J. J., Wood, J. M. (2004) Bacterial osmosensing: roles of membrane structure and electrostatics in lipid-protein and protein-protein interactions. Biochim. Biophys. Acta. 1666, 88-104.

Price-Whelan, A., Poon, C. K., Benson, M. A., Eidem, T. T., Roux, C. M., Boyd, J. M., Dunman, P. M., Torres, V. J., Krulwich, T. A. (2013) Transcriptional profiling of Staphylococcus aureus during growth in $2 \mathrm{M} \mathrm{NaCl}$ leads to clarification of physiological roles for Kdp and $\mathrm{Ktr}^{+}$uptake systems. mBio. 4, e00407-13.

Quintana, I. M., Gibhardt, J., Turdiev, A., Hammer, E., Commichau, F. M., Lee, V. T., Magni, C., Stülke, J. (2019) The KupA and KupB proteins of Lactococcus lactis IL1403 are novel c-di-AMP receptor proteins responsible for potassium uptake. J. Bacteriol. doi: 10.1128/JB.00028-19.

Radeck, J., Fritz, G., Mascher, T. (2017) The cell envelope stress response of Bacillus subtilis: from static signaling devices to dynamic regulatory network. Curr. Genet. 65, 79-90.

Ragland, S. A., Criss, A. K. (2017) From bacterial killing to immune modulation: recent insights into the functions of lysozyme. PLoS Pathog. 13, e1006512.

Raguse, M., Torres, R., Seco E. M., Gándara, C., Ayora, S., Moeller, R., Alonso, J. C. (2017) Bacillus subtilis DisA helps to circumvent replicative stress during spore revival. DNA Repair (Amst.). 59, 57-68.

Rall, T. W., Sutherland, E. W. (1958) Formation of a cyclic adenine ribonucleotide by tissue particles. J. Biol. Chem. 232, 1065-1076.

Rall, T. W., Sutherland, E. W., Wosilait, W. D. (1956) The relationship of epinephrine and glucagon to liver phosphorylase. III. Reactivation of liver phosphorylase in slices and in extracts. J. Biol. Chem. 218, 483-495.

Rao, F., Ji, Q., Soehano, I., Liang, Z. X. (2011) Unusual heme-binding PAS domain from YybT family proteins. J. Bacteriol. 193, 1543-1551. 
Rao, F., See, R. Y., Zhang, D., Toh, D. C., Ji, Q., Liang, Z. X. (2010) YybT is a signaling protein that contains a cyclic dinucleotide phosphodiesterase domain and a GGDEF domain with ATPase activity. J. Biol. Chem. 285, 473-482.

Reniere, M. L., Whiteley, A. T., Hamilton, K. L., John, S. M., Lauer, P., Brennan, R. G., Portnoy, D. A. (2015) Glutathione activates virulence gene expression of an intracellular pathogen. Nature. 517, 170-173.

Rismondo, J., Gibhardt, J., Rosenberg, J., Kaever, V., Halbedel, S., Commichau, F. M. (2016) Phenotypes associated with the essential diadenylate cyclase $\mathrm{CdaA}$ and its potential regulator CdaR in the human pathogen Listeria monocytogenes. J. Bacteriol. 198, 416-426.

Rocha, R., Teixeira-Duarte, C. M., Jorge, J. M. P., Morais-Cabral, J. H. (2019) Characterization of the molecular properties of $\mathrm{KtrC}$, a second RCK domain that regulates a Ktr channel in Bacillus subtilis. J. Struct. Biol. doi: 10.1016/j.jsb.2019.02.002.

Roelofs, K. G., Wang, J., Sintim, H. O., Lee, V. T. (2011) Differential radial capillary action of ligand assay for high-throughput detection of protein-metabolite interactions. Proc. Natl. Acad. Sci. U.S.A. 108, 15528-15533.

Rojas, E. R., Huang, K. C. (2018) Regulation of microbial growth by turgor pressure. Curr. Opin. Microbiol. 42, 62-70.

Rojas, E., Theriot, J. A., Huang, K. C. (2014) Response of Escherichia coli growth rate to osmotic shock. Proc. Natl. Acad. Sci. U.S.A. 111, 7807-7812.

Rolhion, N., Cossart, P. (2017) How the study of Listeria monocytogenes has led to new concepts in biology. Future Microbiol. 12, 621-638.

Romeo, Y., Bouvier, J., Gutierrez, C. (2007) Osmotic regulation of transcription in Lactococcus lactis: ionic strength-dependent binding of the BusR repressor to the busA promoter. FEBS Lett. 581, 3387-3390.

Rosenberg, J., Dickmanns, A., Neumann, P., Gunka, K., Arens, J., Kaever, V., Stülke, J., Ficner, R., Commichau, F. M. (2015) Structural and biochemical analysis of the essential diadenylate cyclase CdaA from Listeria monocytogenes. J. Biol. Chem. 290, 6596-6606.

Roux, M., Bloom, M. (1990) $\mathrm{Ca}^{2+}, \mathrm{Mg}^{2+}, \mathrm{Li}^{+}, \mathrm{Na}^{+}$, and $\mathrm{K}^{+}$distributions in the headgroup region of binary membranes of phosphatidylcholine and phosphatidylserine as seen by deuterium NMR. Biochemistry. 31, 7077-7089.

Rubin, B. E., Huynh, T. N., Welkie, D. G., Diamond, S., Simkovsky, R., Pierce, E. C., Taton, A., Lowe, L. C., Lee, J. J., Rifkin, S. A., et al. (2018) High-throughput interaction screens illuminate the role of c-di-AMP in cyanobacterial nighttime survival. PLoS Genet. 14, e1007301.

Sambrook, J., Maniatis, T., Fritsch, E. F. (1989) Molecular cloning: a laboratory manual, $2^{\text {nd }}$ Ed., Cold Spring Harbor Laboratory Press, New York.

Santos, J. S., Asmar-Rovira, G. A., Han, G. W., Liu, W., Syeda, R., Cherezov, V., Baker, K. A., Stevens, R. C., Montal, M. (2012) Crystal structure of a voltage-gated $\mathrm{K}^{+}$channel pore module in a closed state in lipid membranes. J. Biol. Chem. 287, 43063-43070.

Santos, J. S., Grigoriev, S. M., Montal, M. (2008) Molecular template for a voltage sensor in a novel $\mathrm{K}^{+}$channel. III. Functional reconstitution of a sensor-less pore module from a prokaryotic Kv channel. J. Gen. Physiol. 132, 651-666.

Sarenko, O., Klauck, G., Wilke, F. M., Pfiffer, V., Richter, A. M., Herbst, S., Kaever, V., Hengge, R. (2017) More than enzymes that make or break cyclic di-GMP - local signaling in the interactome of GGDEF/EAL domain proteins of Escherichia coli. mBio. 8, e01639-17. 
Schär, J., Stoll, R., Schauer, K., Loeffler, D. I., Eylert, E., Joseph, B., Eisenreich, W., Fuchs, T. M., Goebel, W. (2010) Pyruvate carboxylase plays a crucial role in carbon metabolism of extraand intracellularly replicating Listeria monocytogenes. J. Bacteriol. 192, 1774-1784.

Schirmer, F., Ehrt, S., Hillen, W. (1997) Expression, inducer spectrum, domain structure, and function of MopR, the regulator of phenol degradation in Acinetobacter calcoaceticus NCIB8250. J. Bacteriol. 179, 1329-1336.

Schneider, C. A., Rasband, W. S., Eliceiri, K. W. (2012) NIH Image to ImageJ: 25 years of image analysis. Nat. Methods. 9, 671-675.

Schuster, C. F., Bellows, L. E., Tosi, T., Campeotto, I., Corrigan, R. M., Freemont, P., Gründling, A. (2016) The second messenger c-di-AMP inhibits the osmolyte uptake system OpuC in Staphylococcus aureus. Sci. Signal. 9, ra81.

Scortti, M., Monzó, H. J., Lacharme-Lora, L., Lewis, D. A., Vázquez-Boland, J. A. (2007) The PrfA virulence regulon. Microbes. Infect. 9, 1196-1207.

Sesto, N., Koutero, M., Cossart, P. (2014) Bacterial and cellular RNAs at work during Listeria infection. Future Microbiol. 9, 1025-1037.

Sévin, D. C., Sauer, U. (2014) Ubiquinone accumulation improves osmotic-stress tolerance in Escherichia coli. Nat. Chem. Biol. 10, 266-272.

Shen, A., Kamp, H. D., Gründling, A., Higgins, D. E. (2006) A bifunctional O-GlcNAc transferase governs flagellar motility through anti-repression. Genes Dev. 20, 3283-8295.

Silhavy, T. J., Kahne, D., Walker, S. (2010) The bacterial cell envelope. Cold Spring Harb. Perspect. Biol. 2, a000414.

Sleator, R. D., Hill, C. (2002) Bacterial osmoadaptation: the role of osmolytes in bacterial stress and virulence. FEMS Microbiol. Rev. 26, 49-71.

Smith L. T. (1996) Role of osmolytes in adaptation of osmotically stressed and chill-stressed Listeria monocytogenes grown in liquid media and on processed meat surfaces. Appl. Environ. Microbiol. 62, 3088-3093.

Spiering M. J. (2015) Primer on the Immune System. Alcohol Res. 37, 171-175.

Stannek, L. (2015) Control of glutamate homeostasis in the Gram-positive model organism Bacillus subtilis. Dissertation. Georg-August-Universität Göttingen. http://hdl.handle.net/11858/00-1735-0000-0023-9652-B, accessed 04.02.2019.

Stumpe, S., Bakker, E. P. (1997) Requirement of a large $\mathrm{K}^{+}$-uptake capacity and of extracytoplasmic protease activity for protamine resistance of Escherichia coli. Arch. Microbiol. 167, 126136.

Suomi, T., Corthals, G. L., Nevalainen, O. S., Elo, L. L. (2015) Using peptide-level proteomics data for detecting differentially expressed proteins. J. Proteome Res. 14, 4564-4570.

Suomi, T., Elo, L. L. (2017) Enhanced differential expression statistics for data-independent acquisition proteomics. Sci. Rep. 7, 5869.

Sureka, K., Choi, P. H., Precit, M., Delince, M., Pensinger, D. A., Huynh, T. N., Jurado, A. R., Goo, Y. A., Sadilek, M., lavarone, A. T., et al. (2014) The cyclic dinucleotide c-di-AMP is an allosteric regulator of metabolic enzyme function. Cell. 158, 1389-1401.

Tatusov, R. L., Galperin, M. Y., Natale, D. A., Koonin, E. V. (2000) The COG database: a tool for genome-scale analysis of protein functions and evolution. Nucleic Acids Res. 28, 33-36. 
Team, R. D. C. (2014) R: a language and environment for statistical computing. Vienna, Austria: the $\mathrm{R}$ Foundation for Statistical Computing. https://www.R-project.org/, accessed 13.02.2019.

Thongsomboon, W., Serra, D. O., Possling, A., Hadjineophytou, C., Hengge, R., Cegelski, L. (2018) Phosphoethanolamine cellulose: a naturally produced chemically modified cellulose. Science. 359, 334-338.

Thorsing, M., Dos Santos, P. T., Kallipolitis, B. H. (2018) Small RNAs in major foodborne pathogens: from novel regulatory activities to future applications. Curr. Opin. Biotechnol. 49, 120128.

Toledo-Arana, A., Dussurget, O., Nikitas, G., Sesto, N., Guet-Revillet, H., Balestrino, D., Loh, E., Gripenland, J., Tiensuu, T., Vaitkevicius, K., et al. (2009) The Listeria transcriptional landscape from saprophytism to virulence. Nature. 459, 950-956.

Tosi, T., Hoshiga, F., Millership, C., Singh, R., Eldrid, C., Patin, D., Mengin-Lecreulx, D., Thalassinos, K., Freemont, P., Gründling, A. (2019) Inhibition of the Staphylococcus aureus c-di-AMP cyclase DacA by direct interaction with the phosphoglucosamine mutase GImM. PLoS Pathog. 15, e1007537.

Townsley, L., Yannarell, S. M., Huynh, T. N., Woodward, J. J., Shank, E. A. (2018) Cyclic di-AMP acts as an extracellular signal that impacts Bacillus subtilis biofilm formation and plant attachment. mBio. 9, e00341-18.

Valenzuela-García, L. I., Ayala-García, V. M., Regalado-García, A G., Setlow, P., Pedraza-Reyes, M. (2018) Transcriptional coupling (Mfd) and DNA damage scanning (DisA) coordinate excision repair events for efficient Bacillus subtilis spore outgrowth. Microbiologyopen. 7, e00593.

van Leeuwenhoek, A. (1677) Observationes $d$. Anthonii Lewenhoeck, de natis e semine genitali animalculis. Philos. Trans. 12, 1040-1046.

Vázquez-boland, J. A., Kuhn, M., Berche, P., Chakraborty, T., Domi, G., González-Zorn, B., Wehland, J. (2001) Listeria pathogenesis and molecular virulence determinants. Clin. Microbiol. Rev. 14, 584-640.

Vienna, H. W. R. C. T., Austria, (2017, n.d.) Tidyverse: easily install and load the "Tidyverse." R package version 1.2. 1. https://CRAN.R-project.org/package=tidyverse, accessed 13.02.2019.

Vlamakis, H., Chai, Y., Beauregard, P., Losick, R., Kolter, R. (2013) Sticking together: building a biofilm the Bacillus subtilis way. Nat. Rev. Microbiol. 11, 157-168.

Vollmer, W., Joris, B., Charlier, P., Foster S. (2008). Bacterial peptidoglycan (murein) hydrolases. FEMS Microbiol. Rev. 32, 259-286.

Wach, A. (1996) PCR-synthesis of marker cassettes with long flanking homology regions for gene disruptions in Saccharomyces cerevisiae. Yeast. 12, 259-265.

Whatmore, A. M., Reed, R. H. (1990) Determination of turgor pressure in Bacillus subtilis: a possible role for $\mathrm{K}^{+}$in turgor regulation. J. Gen. Microbiol. 136, 2521-2526.

Whiteley, A. T., Garelis, N. E., Peterson, B. N., Choi, P. H., Tong, L., Woodward, J. J., Portnoy, D. A. (2017) c-di-AMP modulates Listeria monocytogenes central metabolism to regulate growth, antibiotic resistance and osmoregulation. Mol. Microbiol. 104, 212-233.

Whiteley, A. T., Pollock, A. J., Portnoy, D. A. (2015) The PAMP c-di-AMP is essential for Listeria monocytogenes growth in rich but not minimal media due to a toxic increase in (p)ppGpp. Cell Host Microbe. 17, 788-798. 
WHO (2014) Antimicrobial resistance: global report on surveillance 2014, World Health Organization (WHO). https://www.who.int/drugresistance/documents/surveillancereport/en/, accessed 22nd January 2019.

Wichgers Schreur, P. J., van Weeghel, C., Rebel, J. M., Smits, M. A., van Putten, J. P., Smith, H. E. (2012) Lysozyme resistance in Streptococcus suis is highly variable and multifactorial. PLoS One. 7, e36281.

Witte, C. E., Whiteley, A. T., Burke, T. P., Sauer, J. D., Portnoy, D. A., Woodward, J. J. (2013) Cyclic di-AMP is critical for Listeria monocytogenes growth, cell wall homeostasis, and establishment of infection. mBio. 4, e00282-13.

Witte, G., Hartung, S., Büttner, K., Hopfner, K. P. (2008) Structural biochemistry of a bacterial checkpoint protein reveals diadenylate cyclase activity regulated by DNA recombination intermediates. Mol. Cell. 30, 167-178.

Wood, J. M. (1999) Osmosensing by bacteria: signals and membrane-based sensors. Annu. Rev. Microbiol. 63, 230-262.

Wood, J. M. (2011) Bacterial osmoregulation: a paradigm for the study of cellular homeostasis. Annu. Rev. Microbiol. 65, 215-238.

Wood, J. M., Bremer, E., Csonka, L. N., Kraemer, R., Poolman, B., van der Heide, T., Smith, L. T. (2001) Osmosensing and osmoregulatory compatible solute accumulation by bacteria. Comp. Biochem. Physiol. A. 130, 437-460.

Woodward, J. J., lavarone, A. T., Portnoy, D. A. (2010) c-di-AMP secreted by intracellular Listeria monocytogenes activates a host type I interferon response. Science. 328, 1703-1705.

Wu, J., Sun, L., Chen, X., Du, F., Shi, H., Chen, C., Chen, Z. J. (2012) Cyclic GMP-AMP is an endogenous second messenger in innate immune signaling by cytosolic DNA. Science. 339, 826-830.

Wurtzel, O., Sesto, N., Mellin, J. R., Karunker, I., Edelheit, S., Bécavin, C., Archambaud, C., Cossart, P., Sorek, R. (2012) Comparative transcriptomics of pathogenic and non-pathogenic Listeria species. Mol. Sys. Biol. 8, 583.

Yan, D., Ikeda, T. P., Shauger, A. E., Kustu, S. (1996) Glutamate is required to maintain the steady-state potassium pool in Salmonella typhimurium. Proc. Natl. Acad. Sci. U.S.A. 93, 6527 6531.

Yang, J., Bai, Y., Zhang, Y., Gabrielle, V. D., Jin, L., Bai, G. (2014) Deletion of the cyclic di-AMP phosphodiesterase gene (cnpB) in Mycobacterium tuberculosis leads to reduced virulence in a mouse model of infection. Mol. Microbiol. 93, 65-79.

Yuan, J., Jin, F., Glatter, T., Sourjik, V. (2017) Osmosensing by the bacterial PhoQ/PhoP twocomponent system. Proc. Natl. Acad. Sci. U.S.A. 114, E10792-E10798.

Zarrella, T. M., Metzger, D. W., Bai, G. (2018) Stress suppressor screening leads to detection of regulation of cyclic di-AMP homeostasis by a Trk family effector protein in Streptococcus pneumoniae. J. Bacteriol. 200, e00045-18.

Zeden, M. S., Schuster, C. F., Bowman, L., Zhong, Q., Williams, H. D., Gründling, A. (2018) Cyclic diadenosine monophosphate (c-di-AMP) is required for osmotic regulation in Staphylococcus aureus but dispensable for viability in anaerobic conditions. J. Biol. Chem. 293, 3180-3200.

Zhang, L., He, Z. G. (2013) Radiation-sensitive gene A (RadA) targets DisA, DNA integrity scanning protein A, to negatively affect cyclic Di-AMP synthesis activity in Mycobacterium smegmatis. J. Biol. Chem. 288, 22426-22436. 
Zhang, L., Li, W., He, Z.-G. (2013) DarR, a TetR-like transcriptional factor, is a cyclic di-AMP-responsive repressor in Mycobacterium smegmatis. J. Biol. Chem. 288, 3085-3096.

Zheng, C., Ma, Y., Wang, X., Xie, Y., Ali, M. K., He, J. (2015) Functional analysis of the sporulationspecific diadenylate cyclase CdaS in Bacillus thuringiensis. Front. Microbiol. 6, 908.

Zhu, B., Stülke, J. (2017) SubtiWiki in 2018: from genes and proteins to functional network annotation of the model organism Bacillus subtilis. Nucleic Acids Res. 46, D743-D748.

Zhu, Y., Pham, T. H., Nhiep, T. H. N., Vu, N. M. T., Marcellin, E., Chakrabortti, A., Wang, Y., Waanders, J., Lo, R., Huston, W. M., et al. (2016) Cyclic-di-AMP synthesis by the diadenylate cyclase CdaA is modulated by the peptidoglycan biosynthesis enzyme GImM in Lactococcus lactis. Mol. Microbiol. 99, 1015-1027. 
Cyclic di-AMP and osmoregulation in Listeria monocytogenes 


\section{Appendix}

Used chemical, materials, equipment, commercial systems, enzymes and bioinformatic tools are listed in Tab. 10.1, 10.2, 10.3, 10.4, 10.5 and 10.6, respectively.

Tab. 10.1 Used chemicals and manufacturer

\begin{tabular}{|c|c|}
\hline Chemicals & Manufacturer \\
\hline 5-bromo-4-chloro-3-indolyl- $\beta$-D-galactopyranoside (X-Gal) & Thermo Scientific, USA \\
\hline Acetonitrile & Roth, Karlsruhe \\
\hline Agar & Roth, Karlsruhe \\
\hline Agarose & Peqlab, Erlangen \\
\hline \multirow{2}{*}{ Amino acids } & AppliChem, Darmstadt \\
\hline & Roth, Karlsruhe \\
\hline Ammonium ferric citrate (CAF) & Sigma-Aldrich, München \\
\hline Ampicillin & AppliChem, Darmstadt \\
\hline Bradford solution Roti ${ }^{\circledR}$ Quant & Roth, Karlsruhe \\
\hline Brain heart infusion $(\mathrm{BHI})$ agar & Sigma-Aldrich, München \\
\hline Brain heart infusion (BHI) broth & Sigma-Aldrich, München \\
\hline Bromphenol blue & Serva, Heidelberg \\
\hline Calcium chloride & Roth, Karlsruhe \\
\hline Casamino acids (CAA) & Sigma-Aldrich, München \\
\hline Dimethyl sulfoxide (DMSO) & Sigma-Aldrich, München \\
\hline Disodium phosphate & Roth, Karlsruhe \\
\hline dNTPs & Thermo Scientific, USA \\
\hline Erythromycin & Sigma-Aldrich, München \\
\hline Ethylenediaminetetraacetic acid (EDTA) & Sigma-Aldrich, München \\
\hline Glucose & Merck, Darmstadt \\
\hline Glycerol & Merck, Darmstadt \\
\hline HDGreen $^{\mathrm{TM}}$ Plus & Intas, Göttingen \\
\hline Höchst 33342 & Sigma-Aldrich, München \\
\hline Horse serum & Gibco, Darmstadt \\
\hline Hydrochloric acid ( $\mathrm{HCl})$ & ChemSolute, Renningen \\
\hline Isopropyl $\beta$-D-1-thiogalactopyranoside (IPTG) & Roth, Karlsruhe \\
\hline Kanamycin & Roth, Karlsruhe \\
\hline Magnesium chloride & AppliChem, Darmstadt \\
\hline Magnesium sulfate & Roth, Karlsruhe \\
\hline Manganese chloride & AppliChem, Darmstadt \\
\hline Methanol & AppliChem, Darmstadt \\
\hline Penicillin G & Serva, Heidelberg \\
\hline Polysorbate 20 (Tween 20) & Sigma-Aldrich, München \\
\hline Potassium chloride & AppliChem, Darmstadt \\
\hline Potassium phosphate & Roth, Karlsruhe \\
\hline Roti $^{\oplus}$-Chloroform/Isoamyl alcohol (24:1) & Roth, Karlsruhe \\
\hline Roti $^{\circledR}$-Phenol/Chloroform/Isoamyl alcohol $(25: 24: 1)$ & Roth, Karlsruhe \\
\hline Sodium chloride & Roth, Karlsruhe \\
\hline Sodium dodecyl sulfate (SDS) & Roth, Karlsruhe \\
\hline
\end{tabular}




\begin{tabular}{ll}
\hline Chemicals & Manufacturer \\
\hline Sodium hydroxide $(\mathrm{NaOH})$ & Roth, Karlsruhe \\
Tris(hydroxymethyl)aminomethane & Roth, Karlsruhe \\
Tryptone & Oxoid, Heidelberg \\
Yeast extract & Oxoid, Heidelberg \\
$\lambda$ phage DNA & Thermo Scientific, USA \\
\hline
\end{tabular}

Tab. 10.2 Auxiliary materials

\begin{tabular}{|c|c|}
\hline Auxiliary materials & Manufacturer \\
\hline Centrifuge beaker & Beckmann, München \\
\hline Cuvettes (microliter, plastic) & Sarstedt, Nümbrecht \\
\hline Erlenmeyer flasks (100 - $2000 \mathrm{ml})$ & Duran, Wertheim/Main \\
\hline Falcon ${ }^{\circledR}$ tubes (15 ml, $\left.50 \mathrm{ml}\right)$ & Sarstedt, Nümbrecht \\
\hline Gene Amp Reaction Tubes (PCR) & Perkin Elmer, Weiterstadt \\
\hline Glass beads $\varnothing=0.1 \mathrm{~mm}$ & BioSpec Products, USA \\
\hline Glass pipets & Brand, Wertheim \\
\hline Inoculation loops & Sarstedt, Nümbrecht \\
\hline Membrane filter NC45 (0.2 $\mu \mathrm{m}$ pore size) & Schleicher und Schüll, Dassel \\
\hline Microtest Plate 96-Well,F & Sarstedt, Nümbrecht \\
\hline Micro tubes (1.5 ml, $2 \mathrm{ml})$ & Greiner, Nürtingen \\
\hline Microliter pipets $(2 \mu \mathrm{l}, 20 \mu \mathrm{l}, 200 \mu \mathrm{l}, 1000 \mu \mathrm{l}, 5000 \mu \mathrm{l})$ & Eppendorf, Hamburg \\
\hline Micropulser ${ }^{T M}$ electroporation cuvettes $(0.1 \mathrm{~cm})$ & Bio-Rad Laboratories, München \\
\hline Pasteur pipets & VWR International, Darmstadt \\
\hline \multirow[t]{2}{*}{ Petri dishes } & Greiner, Nürtingen \\
\hline & Greiner, Nürtingen \\
\hline \multirow[t]{2}{*}{ Pipet tips } & Eppendorf, Hamburg \\
\hline & Sarstedt, Nümbrecht \\
\hline Screw cap micro tubes $(1.5 \mathrm{ml}, 2 \mathrm{ml})$ & Sarstedt, Nümbrecht \\
\hline Single-use syringes ( $5 \mathrm{ml}, 10 \mathrm{ml}, 50 \mathrm{ml}$ ) & Becton Dickinson Drogheda, Ireland \\
\hline Tissue flasks $\left(25 \mathrm{~cm}^{2}, 75 \mathrm{~cm}^{2}, 150 \mathrm{~cm}^{2}, 300 \mathrm{~cm}^{2}\right)$ & TPP, Switzerland \\
\hline
\end{tabular}

\section{Tab. 10.3 Equipment}

\begin{tabular}{ll}
\hline Equipment & Manufacturer \\
\hline Autoclaves & Zirbus, Bad Grund \\
& Thermo Scientific, USA \\
Centrifuges & Heraeus Christ, Osterode \\
Clean bench HeraSafe & Thermo Scientific, USA \\
Electroporation device Micro Pulser ${ }^{\mathrm{TM}}$ & Heraeus Christ, Osterode \\
Epoch2 multiwell platereader & Bio-Rad Laboratories, USA \\
Fluorescence microscope Axioskop 40 FL+; Camera AxioCam MRm & BioTek Instruments, USA \\
Gel electrophoresis device EasyCast ${ }^{\mathrm{TM}}$ Mini gel system & Carl Zeiss, Jena \\
Heating block DB3 & Peqlab, Erlangen \\
High accuracy scale & Waasetec, Göttingen
\end{tabular}




\begin{tabular}{|c|c|}
\hline Equipment & Manufacturer \\
\hline Horizontal shaker 3006 & GFL, Burgwedel \\
\hline Ice machine & Ziegra, Isernhagen \\
\hline Incubation water bath 1083 & GFL, Burgwedel \\
\hline Incubator Functionline & Heraeus Christ, Osterode \\
\hline Incubator shaker Innova ${ }^{\circledR} 2300$ & New Brunswick, Neu-Isenburg \\
\hline Incubator shaker Innova ${ }^{\circledR} 40$ & New Brunswick, Neu-Isenburg \\
\hline Magnetic stirrer & JAK Werk, Staufen \\
\hline Molecular Imager ${ }^{\circledast}$ Gel Doc ${ }^{\mathrm{TM}}$ XR+ & Bio-Rad Laboratories, USA \\
\hline Nanodrop ${ }^{\circledR}$ ND-1000 & Thermo Scientific, USA \\
\hline pH-meter Calimatic & Knick, Berlin \\
\hline Refrigarated centrifuge PrimoR & Heraeus Christ, Osterode \\
\hline Scale Sartorius universal & Sartorius, Göttingen \\
\hline Spectral photometer Libra S21 & Biochrom, Cambridge \\
\hline Standard power pac & Bio-Rad Laboratories, USA \\
\hline Stereo fluorescence microscope Lumar V.12 & Carl Zeiss, Jena \\
\hline Synergy Mx multiwell platereader & BioTek Instruments, USA \\
\hline Thermoblock TermoStat Plus & Eppendorf, Hamburg \\
\hline Thermoblock Thermo & Eliwell, Pieve d'Alpago \\
\hline Thermocycler LabCycler & SensorQuest, Göttingen \\
\hline Thermocycler Tpersonal & Biometra, Göttingen \\
\hline Thermomixer compact & Eppendorf, Hamburg \\
\hline TissueLyser II & Qiagen, Hilden \\
\hline Vacuum centrifuge SPD111V SpeedVac ${ }^{\mathrm{TM}}$ Concentrator & Thermo Scientific, USA \\
\hline Vacuum pump VacuGene $\mathrm{T}^{\mathrm{TM}}$ & GE Healthcare Bio-Sciences AB, Sweden \\
\hline Vortex Genie 2 & Bender \& Hobeing AG, Zürich \\
\hline Water distillation plant & Millipore, Schwalbach \\
\hline
\end{tabular}

Tab. 10.4 Commercial systems

\begin{tabular}{ll}
\hline Commercial systems & Manufacturer \\
\hline Nucleospin $^{\circledast}$ Plasmid Kit & Macherey-Nagel, Düren \\
Nucleospin $^{\circledast}$ Microbial DNA Kit & Macherey-Nagel, Düren \\
QIAquick $^{\circledR}$ PCR-purification kit & Qiagen, Venlo \\
peqGOLD Gel Extraction Kit $^{\text {pat }}$ & PeqLab, Erlangen \\
\hline
\end{tabular}

\section{Tab. 10.5 Enzymes}

\begin{tabular}{ll}
\hline Enzymes & Manufacturer \\
\hline FastAP $^{\text {TM }}$ (alkaline phosphatase) & Thermo Scientific, USA \\
FastDigest $^{\text {TM }}$ restriction endonucleases & Thermo Scientific, USA \\
Lysozyme from chicken egg white $^{\text {PM }}$ & Serva, Heidelberg \\
Phusion $^{\text {TM }}$ DNA polymerase & Fermentas, St. Leon-Rot \\
RNaseA & AppliChem, Darmstadt \\
T4- DNA ligase & Thermo Scientific, USA \\
\hline
\end{tabular}


Tab. 10.6 Bioinformatic tools, software and web services

\begin{tabular}{|c|c|c|}
\hline Program/Service & Producer & Application \\
\hline AxioVision Rel 4.8 .2 & Carl Zeiss & $\begin{array}{l}\text { Microscopic analysis of single } \\
\text { cells }\end{array}$ \\
\hline $\begin{array}{l}\text { BLAST } \\
\text { http://blast.ncbi.nlm.nih.gov/ }\end{array}$ & National Institutes of Health, USA & BLAST searches \\
\hline Galaxy S9+ & Samsung & Imaging \\
\hline Gen5 02.09.2001 & BioTek Instruments & $\begin{array}{l}\text { Incubation and measurement } \\
\text { of } 96 \text {-well plates }\end{array}$ \\
\hline Geneious ${ }^{\circledR}$ Prime 2019.0 .4 & Biomatters Ltd. & $\begin{array}{l}\text { Genetic analysis, primer de- } \\
\text { sign, analysis of sequence data }\end{array}$ \\
\hline Image Lab 3.0.1 & GelDoc $^{\mathrm{TM}} \mathrm{XR}+$ & Imaging and analysis \\
\hline Image Lab ${ }^{\mathrm{TM}}$ software (Gel Doc) & Bio-Rad & $\begin{array}{l}\text { Analysis of UV-Vis signals and } \\
\text { image processing of agar plates }\end{array}$ \\
\hline ImageJ 1.48 & National Institutes of Health, USA & $\begin{array}{l}\text { Processing of fluorescence mi- } \\
\text { croscopy images }\end{array}$ \\
\hline $\begin{array}{l}\text { KEGG http://www.ge- } \\
\text { nome.jp/kegg/ }\end{array}$ & Kanehisa Laboratories & $\begin{array}{l}\text { Genome sequence analysis and } \\
\text { information about genes }\end{array}$ \\
\hline $\begin{array}{l}\text { Listeriomics https://listeri- } \\
\text { omics.pasteur.fr }\end{array}$ & Institut Pasteur, France & $\begin{array}{l}\text { Information about genes and } \\
\text { gene expression in L. mono- } \\
\text { cytogenes }\end{array}$ \\
\hline $\begin{array}{l}\text { ListiList http://genolist.pas- } \\
\text { teur.fr/ListiList/ }\end{array}$ & Institut Pasteur, France & $\begin{array}{l}\text { BLAST searches and genome } \\
\text { analysis in Listeria monocyto- } \\
\text { genes }\end{array}$ \\
\hline $\begin{array}{l}\text { ListiWiki http://listiwiki.uni- } \\
\text { goettingen.de/ }\end{array}$ & $\begin{array}{l}\text { General Microbiology, Georg-August-University, } \\
\text { Göttingen }\end{array}$ & $\begin{array}{l}\text { Information about genes and } \\
\text { proteins in L. monocytogenes }\end{array}$ \\
\hline Microsoft ${ }^{\circledR}$ Office 2016 & Microsoft ${ }^{\circledR}$ Inc. & $\begin{array}{l}\text { Processing of images, text and } \\
\text { data }\end{array}$ \\
\hline $\begin{array}{l}\text { NEBcutter } \\
\text { http://tools.neb.com/NEBcut- } \\
\text { ter2/ }\end{array}$ & New England Biolabs, Inc. & Restriction site analysis \\
\hline $\begin{array}{l}\text { OligoCalc } \\
\text { http://www.basic.northwest- } \\
\text { ern.edu/biotools/oligocalc.html }\end{array}$ & $\begin{array}{l}\text { Department of Molecular Genetics and Bio- } \\
\text { chemistry, University of Pittsburgh School of } \\
\text { Medicine }\end{array}$ & Oligonucleotide analysis \\
\hline $\begin{array}{l}\text { PubMed } \\
\text { http://www.ncbi.nlm.nih.gov/ }\end{array}$ & National Institutes of Health, Bethesda, USA & Literature research \\
\hline $\begin{array}{l}\text { Reverse Complement } \\
\text { http://www.bioinformat- } \\
\text { ics.org/sms/rev_comp.html }\end{array}$ & $\begin{array}{l}\text { Bioinformatics Organization, Inc., Massachu- } \\
\text { setts, USA }\end{array}$ & $\begin{array}{l}\text { Formation of reverse-comple- } \\
\text { ment sequences }\end{array}$ \\
\hline STRING http://string-db.org & $\begin{array}{l}\text { String consortium CRP, EMBL, KU, SIB, TUD and } \\
\text { UZH }\end{array}$ & $\begin{array}{l}\text { Information about protein-pro- } \\
\text { tein interactions }\end{array}$ \\
\hline $\begin{array}{l}\text { SubtiWiki http://subtiwiki.uni- } \\
\text { goettingen.de/ }\end{array}$ & $\begin{array}{l}\text { General Microbiology, Georg-August-University, } \\
\text { Göttingen }\end{array}$ & $\begin{array}{l}\text { Information about genes and } \\
\text { proteins in } B \text {. subtilis }\end{array}$ \\
\hline $\begin{array}{l}\text { UniProt http://www.uni- } \\
\text { prot.org/ }\end{array}$ & UniProt consortium EMBL-EBI, SIB, PIR & $\begin{array}{l}\text { Information about protein } \\
\text { functions, sequences and do- } \\
\text { mains }\end{array}$ \\
\hline
\end{tabular}




\section{Curriculum vitae}

Name:

Date of birth:

Place of birth:

Nationality:

\section{Education}

$10 / 2015-04 / 2019$

$10 / 2013-08 / 2015$

$10 / 2010-08 / 2013$

$08 / 2007-06 / 2010$
Johannes Gibhardt

19.01.1991

Kassel, Germany

German

PhD student in the GGNB Program Microbiology and Biochemistry

Department of General Microbiology

Institute of Microbiology and Genetics

Georg-August-Universität Göttingen, 37073 Göttingen, Germany

Doctoral Thesis Project: "Cyclic di-AMP homeostasis and osmoregulation in Listeria monocytogenes"

Master degree course Microbiology and Biochemistry

Georg-August-Universität Göttingen, 37073 Göttingen, Germany

Master thesis: "Cyclic Di-AMP Metabolism and Osmoregulation in Listeria monocytogenes"

Degree: M. Sc.

Final grade: 1.1 , passed with distinction

\section{Bachelor degree course Biology}

Georg-August-Universität Göttingen, 37073 Göttingen, Germany

Major field of study: Molecular Biosciences

Bachelor thesis: "Etablierung eines Screening-Systems zur Identifizierung neuer Faktoren, die die Aktivität des Virulenzfaktors PrfA von Listeria monocytogenes beeinflussen"

Degree: B. Sc.

Final grade: 1.5 , passed with distinction

Abitur

Georg-Christoph-Lichtenberg-Schule, 34132 Kassel, Germany

Abitur certificate grade 2.6 
Cyclic di-AMP and osmoregulation in Listeria monocytogenes

\title{
Internships
}

$09 / 2014-11 / 2014$

\author{
Internship at the Institute for Medical Microbiology \\ Universitätsmedizin Göttingen (UMG), 37099 Göttingen, Ger- \\ many \\ Topic: Characterization of clinical isolates of Clostridium difficile \\ Reference: Dr. Katrin Gunka \\ E-Mail:kgunka@gwdg.de
}

$11 / 2014-12 / 2014$

\author{
Internship at the Robert Koch Institute \\ Robert Koch-Institut (RKI), 38855 Wernigerode, Germany \\ Topic: Development of a counter selection system for genetic \\ modifications of Listeria monocytogenes \\ Reference: Dr. Sven Halbedel \\ E-Mail: halbedels@rki.de
}

\section{List of publications}

Quintana, I. M., Gibhardt, J., Turdiev, A., Hammer, E., Commichau, F. M., Lee, V. T., Magni, C., Stülke, J. (2019) The KupA and KupB proteins of Lactococcus lactis IL1403 are novel c-di-AMP receptor proteins responsible for potassium uptake. J. Bacteriol. doi: 10.1128/JB.00028-19.

Hauf, S., Herrmann, J., Miethke, M., Gibhardt, J., Commichau, F. M., Müller, R., Fuchs, S., Halbedel, S. (2019) Aurantimycin resistance genes contribute to survival of Listeria monocytogenes during life in the environment. Mol. Microbiol. doi: 10.1111/mmi.14205.

Wicke, D., Schulz, L. M., Lentes, S., Scholz, P., Poehlein, A., Gibhardt, J., Daniel, R., Ischebeck, T., Commichau, F. M. (2019) Identification of the first glyphosate transporter by genomic adaptation. Environ. Microbiol. doi: 10.1111/1462-2920.14534.

Commichau, F. M., Gibhardt, J., Halbedel, S., Gundlach, J., Stülke, J. (2018) A delicate connection: c-di-AMP affects cell Integrity by controlling osmolyte transport. Trends Microbiol. 26, $175-185$.

Gundlach, J., Herzberg, C., Kaever, V., Gunka, K., Hoffmann, T., Weiß, M., Gibhardt, J., Thürmer, A., Hertel, D., Daniel, R., Bremer, E., Commichau, F. M., Stülke, J. (2017) Control of potassium homeostasis is an essential function of the second messenger cyclic di-AMP in Bacillus subtilis. Sci. Signal. 10, eaal3011.

Großhennig, S., Ischebeck, T., Gibhardt, J., Busse, J., Feussner, I., Stülke, J. (2016) Hydrogen sulfide is a novel potential virulence factor of Mycoplasma pneumoniae: characterization of the unusual cysteine desulfurase/desulfhydrase HapE. Mol. Microbiol. 100, 42-54.

Rismondo, J., Gibhardt, J., Rosenberg, J., Kaever, V., Halbedel, S., Commichau, F. M. (2016) Phenotypes associated with the essential diadenylate cyclase $\mathrm{CdaA}$ and its potential regulator CdaR in the human pathogen Listeria monocytogenes. J. Bacteriol. 198, 416-426. 